\title{
A DESCRIPTION OF IMPERIAL VALLEY, CALIFORNIA FOR THE ASSESSMENT OF IMPACTS OF GEOTHERMAL ENERGY DEVELOPMENT
}

\author{
bavid Layton \\ Donald Ermak
}

August 26,1976

Prepared for U.S. Energy Research \& Development

Administration under contract No. W-7405-Eng-48

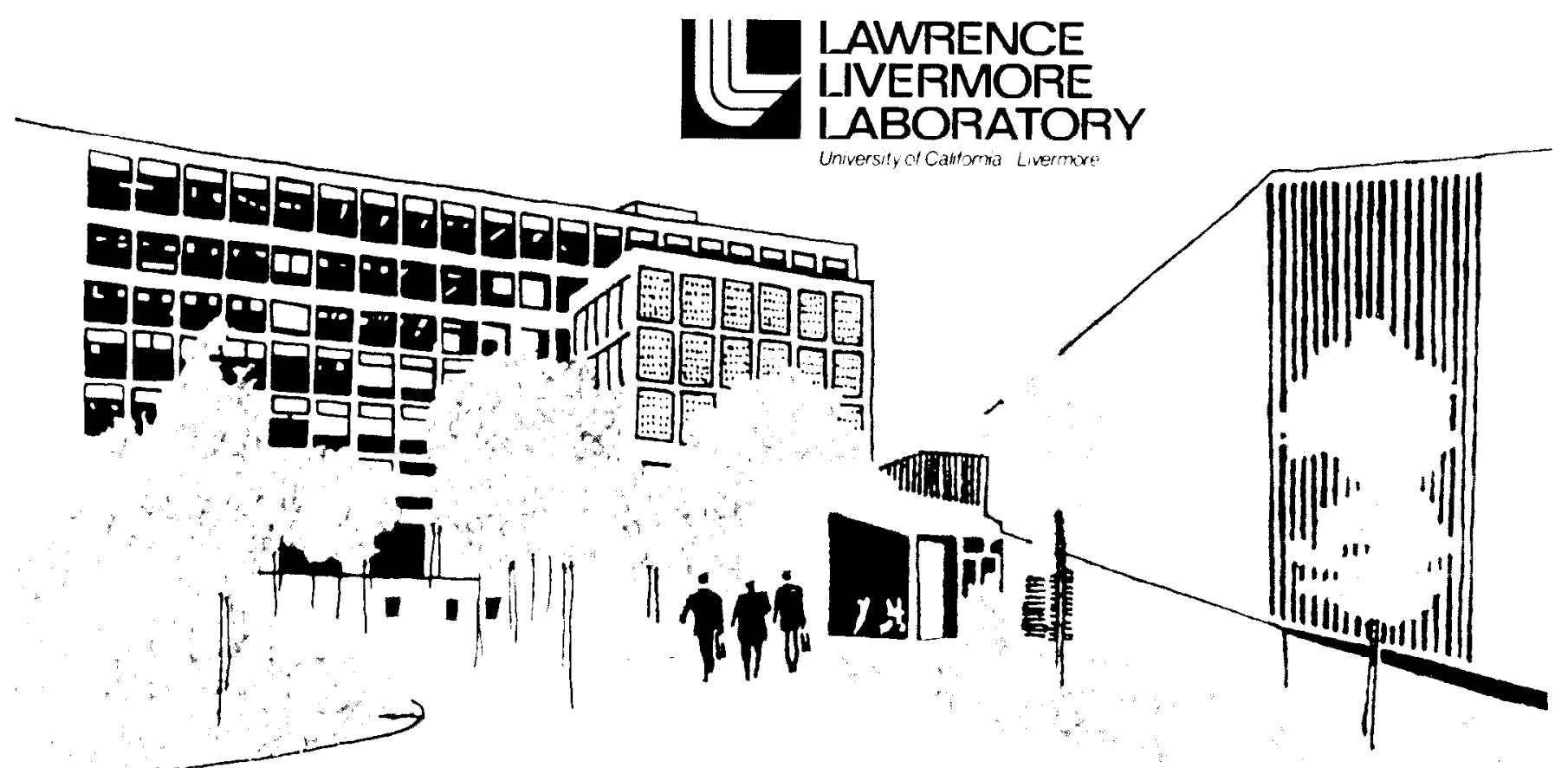




\section{DISCLAIMER}

This document was prepared as an account of work sponsored by an agency of the United States Government. Neither the United States Government nor the University of California nor any of their employees, makes any warranty, express or implied, or assumes any legal liability or responsibility for the accuracy, completeness, or usefulness of any information, apparatus, product, or process

disclosed, or represents that its use would not infringe privately owned rights. Reference herein to any specific commercial product, process, or service by trade name, trademark, manufacturer, or otherwise, does not necessarily constitute or imply its endorsement, recommendation, or favoring by the United States Government or the University of California. The views and opinions of authors expressed herein do not necessarily state or reflect those of the United States Government or the University of California, and shall not be used for advertising or product endorsement purposes. 


\section{近 \\ LAWRENCE LIVERMORE LABORATORY \\ University of Calfornia /Livermore, California, 94550}

UCRL-52121

\section{A DESCRIPTION OF IMPERIAL VALLEY, CALIFORNIA FOR THE ASSESSMENT OF IMPACTS OF GEOTHERMAL ENERGY DEVELOPMENT}

David Layton and Dona1d Ermak

M.S. date: August 26, 1976 



\section{Table of Contents}

Preface . . . . . . . . . . . . . . . . . . . . . . 1

Section 1. Introduction and Summary . . . . . . . . . . . . . . 3

Section 2. Land and Air Resources (Donald Ermak, Mary Buchanan, and David Layton) . . . . . . . . . . . . . . . 7

2.1 Geographical Overview . . . . . . . . . . . . . . . . 7

2.2 Geology . . . . . . . . . . . . . . . . . . . . . . 9

Sedimentary Rocks . . . . . . . . . . . . . . . . . . 9

Structure and Tectonics . . . . . . . . . . . . . . . 9

2.3 Climate . . . . . . . . . . . . . . . . . . . . . 11

2.4 Meteorological Conditions . . . . . . . . . . . . . . . 14

2.5 Air Quality . . . . . . . . . . . . . . . . . . 17

Section 3. Water Resources (David Layton) . . . . . . . . . . . . 22

3.1 Ground Water . . . . . . . . . . . . . . . . 22

3.2 Ground Water Quality . . . . . . . . . . . . . . . 23

3.3 Surface Water . . . . . . . . . . . . . . . . . 25

3.4 Surface Water Quality . . . . . . . . . . . . . . . 28

3.5 Salton Sea . . . . . . . . . . . . . . . . . . 29

Scction 4. Biological Resources (J. R. Kercher and Mary Buchanan) . . . 34

4.1 Agriculture . . . . . . . . . . . . . . . . . . 34

Crops . . . . . . . . . . . . . . . . . . 34

Soils and Salinity . . . . . . . . . . . . . . . . 43

Salinity Control Practices and Associated Problems . . . . . 45

Livestock Production . . . . . . . . . . . . . . . . 47

Weed Control, Pest Control, and Fertilizer . . . . . . . . 47

4.2 Ecology of the Salton Sea Quasi-Marine Ecosystem . . . . . . . 51

History of Ecosystem . . . . . . . . . . . . . . . 55

Food Web Structure . . . . . . . . . . . . . . . 77

Abnormalities . . . . . . . . . . . . . . . . . 81

4.3 Endangered Species and Wildlife Refuges . . . . . . . . . . . 82

Wildlife Refuges . . . . . . . . . . . . . . . . 83

Endangered Species . . . . . . . . . . . . . . . . 85

California Brown Pelican . . . . . . . . . . . . 85

Southern Bald Eagle . . . . . . . . . . . . . . 85

American Peregrine Falcon . . . . . . . . . . . . 86 
California Least Tern . . . . . . . . . . . . 86

Yuma Clapper Rail . . . . . . . . . . . . . . 87

4.4 Vegetative Communities and Vildlife . . . . . . . . . . . 88

Salton Sea KGRA . . . . . . . . . . . . . . . . 88

Glamis KGRA . . . . . . . . . . . . . . . . . . 88

Dunes KGRA . . . . . . . . . . . . . . . . . . 89

East Mesa KGRA . . . . . . . . . . . . . . . . 89

Section 5. Geothermal Resources (Donald Ermak and Mary Buchanan) • • 94

5.1 Resource Identification . . . . . . . . . . . . . . 94

5.2 Estimation of Geothermal Energy Resoucce . . . . . . . . . . 96

5.3 Physical and Chemical Characterization of the Geothermal

Fluids . . . . . . . . . . . . . . 98

Geothermal Reservoir Temperature and Pressure . . . . . . . . 99

Physical Characteristics at the Vell Head . . . . . . . . 100

Chemical Composition of the Brines . . . . . . . . . . . 101

Overview of Geothermal Development . . . . . . . . . . . 103

Noncondensable Gases . . . . . . . . . . . . . . . 103

5.4 Overview of Geothermal Development . . . . . . . . . . . 103

Section 6. County Economic Characterization (kendall Haven) . . . . . 117

6.1 County Economic Composition . . . . . . . . . . . . . 117

Economic Sector Description . . . . . . . . . . . . 123

Multiplier Effects . . . . . . . . . . . . . . 124

6.2 Agricultural Sector Analysis . . . . . . . . . • . . . • 129

6.3 Imperial County Employment and Labor Force . . . . . . . . 146

Nonagricultural Employment . . . . . . . . . . . . . . 146

Unemployment Characteristics and Current Employment

Prospects . . . . . . . . . . . . . . . 147

Mexican Labor Force in Imperial County . . . . . . . . . 152

Imperial County Agricultural Employment . • • • • • • • 153

6.4 Imperial County Electrical Energy Buciget . . . . . • . . • • • 154

6.5 Imperial County Economic Characterization and Comparison to

Other California Countries . . . . . . . . . . 156

6.6 Subcounty Economic Analysis . . . . . . . . . . . . . . . 171

Agriculture . . . . . . . . . . . . . . . . . 171

Sector Intracounty Distribution . . . . . . . . . . . . 171

E1 Centro Economy . . . . . . . . . . . . . • • • • 173 
Section 7. County Fiscal Characterization (Kendall Haven) . . . . . . . 177

7.1 Fiscal Budget Analysis . . . . . . . . . . . . . . . . 177

7.2 County Tax Structure . . . . . . . . . . . . . . . . 183

7.3 County School Districts and Budgets . . . . . . . . . . . . 188

7.4 Imperial County Land Üse Planning . . . . . . . . . . . . . . 195

Zoning Ordinances . . . . . . . . . . . . . . . . . . 196

Ultimate Land Use Plan . . . . . . . . . . . . . . . 196

Geothermal Element . . . . . . . . . . . . . . 196

7.5 Intracounty Fiscal Description . . . . . . . . . . . . . 199

Municipal Services . . . . . . . . . . . . . . . . 199

Brawley . . . . . . . . . . . . . . . . . . 199

Calexico ........................ 200

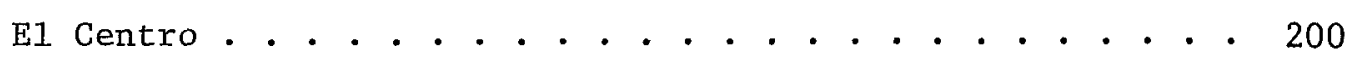

Holtville . . . . . . . . . . . . . . . . 201

Federal Governmental Influence Within the County . . . . . . . 201

Section 8. Social Characterization of Imperial County (Kendall Haven) . 205

8.1 Imperial County Sociological Description . . . . . . . . . . 205

8.2 Intracounty Sociological Description . . . . . . . . . . . . . 214

Intracounty Populations . . . . . . . . . . . . . . 214

Section 9. Geothermal Laws (James Wharton and David-Layton) . . • . . . 216

9.1 Introduction . . . . . . . . . . . . . . . . . . . . 216

9.2 Federal Law on Geothermal Resources . . . . . . . . . . . . . 216

Federal Leases . . . . . . . . . . . . . . . . 216

Federal Regulations . . . . . . . . . . . . . . . 217

Federal Geothermal Loan Guaranty Program . . . . . . . . . . 218

Federal Licenses and Permits . . . . . . . . . . . . . 219

9.3 California State Laws on Geothermal Resources . . . . . . . . . 219

State Geothermal Leases . . . . . . . . . . . . . . . 219

Statutes on Geothermal Wells . . . . . . . . . . . . . 220

California Regulation of Geothermal Wells . . . . . . . . . 221

Geothermal Powerplant Siting . . . . . . . . . . . . . 222

9.4 Local Laws of Imperial County . . . . . . . . . . . . . . 223 



\section{A DESCRIPTION OF IMPERIAL VALLEY, CALIFORNIA FOR THE ASSESSMENT OF IMPACTS OF GEOTHERMAL ENERGY DEVELOPMENT}

\section{Preface}

This report is part of a research effort at the Lawrence Livermore Laboratory known as the Imperial Valley Environmental Project. It is sponsored by the Assistant Administrator for Environment and Safety of the U.S. Energy Research and Development Administration. The project is designed to ensure that the development of geothermal resources proceeds on an environmentally sound basis. To carry out that objective, the project includes the following research groups: Air Quality,
Ecosystem Quality, Water Quality, Subsidence and Seismicity, Health Effects, Socioeconomic Effects, and Integrated Assessment. The background research providing descriptive material on the valley was done under the auspices of the Integrated Assessment group whose special research responsibilities include the evaluation of relevant environmental impacts, the development of alternative geothermal scenarios, data management, and the timely transfer of information to decision makers.

\footnotetext{
Abstract

Impending geothermal development in the Imperial Valley of California has raised concern over the possible impacts of such development. As an initial step in impact assessment of geothermal projects, relevant features of the valley's physical and human environments are described. Particular attention is placed on

features that may either influence development or be affected by it. Major areas of consideration include the valley's physical resources (i.e., land, air, water, and biological resources), economic, fiscal, and social characteristics of Imperial County, and geothermal laws.
} 



\section{Section 1 \\ Introduction and Summary}

A warm climate, good soils, and imported watet combine to make the Imperial Valley of California a place of intense agricultural activity. And now, with increased interest in the geothermal resources underlying the valley, the area faces the prospect of a new industry for the production of electricity, and perhaps water, from geothermal fluids. The development of geothermal resources, nevertheless, will be accompanied by various impacts on the valley's physical and human environments that must be carefully identified and assessed. Sections 2 through 5 of our report describe the valley's natural resources; Sections 6 through 8 review economic, fiscal, and social characteristics of Imperial County; and Section 9 describes geothermal laws that may affect geothermal projects. The principal features of the valley presented in these sections are summarized below.

LAND AND AIR RESOURCES

The Imperial Valley occupies the lowest part of the Colorado Desert. It receives water from the Colorado River to support about 475,000 acres of irrigated lands. The waste waters from these lands help sustain the
Salton Sea, California's largest inland body of water. Geologically the valley is characterized by earthquakes, active faults, and natural subsidence. Hot summers, mild winters, and an average annual rainfall of under 3 inches define its climate; stable atmospheric conditions, westerly winds, and night time inversions are important meteorological features. The most prominent characteristic of the air quality is the high levels of suspended particles that exceed federal standards.

\section{WATER RESOURCES}

Nearly 3 million acre-feet (af) of Colorado River water are diverted to the valley each year by the Imperial Irrigation District. To distribute that water and remove unwanted waste waters, over 3,000 miles of canals and drainage ditches cover the valley. Waste waters entering the Salton Sea have caused its level to rise for many years, and the sea is now at its highest elevation in recent years. Surface water salinity ranges from about $900 \mathrm{ppm}$ total dissolved solids (TDS) in the A11 American Canal to almost 39,000 ppm in the Salton Sea. Sediments underlying the valley contain more than a billion acrefeet of ground water having salinity 
less than 35,000 ppm. Natural

recharge in this arid region is quite low, yet millions of acre-feet have been added to shallow aquifers from canal seepage.

\section{BIOLOGICAL RESOURCES}

There is an extensive, irrigated agricultural region, a quasi-marine inland saltern ecosystem, state and federal game reserves with many shorebirds and waterfowl, five endangered species of birds, freshwater and riparian ecosystems, and extensive desert communities in the Imperial Valley. Agricultural lands produced gross sales in 1974 of $\$ 557$ million of which $\$ 155$ million was in livestock and dairy products, $\$ 284$ million in field crops, and $\$ 103$ million in vegetable crops. In acreage harvested, the most important crops are alfalfa, wheat, cotton, sugar beets, lettuce, sorghum, pasture ryegrass, cantaloupes, carrots, barley, asparagus, onions, and tomatoes. The unique climate is such that most vegetable crops grow in the winter months and most field crops grow in the spring and summer months. Soil salinity problems are important in the valley and require large amounts of Colorado River water for leaching and an extensive underground pipe drainage system to carry off water and dissolved salts. The leached salts are eventually emptied into the
Salton Sea. Of the many attempts to introduce new species into the Sca, only a few have been successful. Curm rently there are eight fish and seven invertebrate species. The sea his high levels of nutrients that cratc an unusually high productivity $(0.75$ $\mathrm{g} / \mathrm{m}^{3} /$ day of carbon fixation). Extensive shorebird (35 species) and watexfow1 (it7 species) (excluding swans, ducks, geese, cranes, and rails) populations exist around the Salton Sea especially in the state and federal game refuges. The Salton Sea i: on the Parific Flyway and hosts large migratory populations (a total of 9.7 million waterfowl use days in 1971 for the Salton Sea National Vildlife Refuge). Outside the irrigated areas in the Valley lies the Sonoran Desert. Typical desert communities of creosote brush, sage, mesquite, ironwood, and desert willow dominate the Glamis, East Mesa, and Dunes Known Geothermal Resource Areas (KGRAs). In these KGRAs some areas are dominated by dune communities of very sparse vegetation and a shifting, sandy soil. The balance of the area in these KGRAs is mixed bajada (alluvial fan) communities or creosote communities.

GOETHERIALI RESOURCES

As early as 1927, efforts vere made to develop the geothermal resources in the Imperial Valley. Mineral extraction, carbon dioxide 
recovery, and power production have all been attempted, but with limited success. Some of the current geothermal activities are electric power conversion research, impact studies of proposed geothermal projects, baseline environmental studies, and exploratory drilling. Of the 6 KGRAs, only the Salton Sea, Heber, East Mesa, and Brawley areas are expected to be developed. Estimates of their total electrical potential are under 5,000 MW for 30 years. The Salton Sea KGRA has the greatest energy potential because of its high down-hole temperatures (average of $286^{\circ} \mathrm{C}$ ), yet it may be the hardest to develop since brines found there average over 200,000 ppm TDS. In comparison, the Heber KGRA has geothermal fluids of around 20,000 ppm TDS, while those of the East Mesa KGRA are about 2,100 ppm TDS. Other relevant characteristics of the geothermal fluids in the valley include a steam content of between 10 to $25 \%$ by weight and the presence of minor amounts of noncondensable gases.

\section{COUNTY ECONOMIC CHARACTERIZATION}

The Imperial County economy is dominated by agriculture, its associated support services, and product processing. Agricultural activities are dominated by beef cattle and by general field crops. In $1974,85.9 \%$ of the total valley acreage was planted in field crops with a total dollar value of $\$ 284$ million. There was a decline in beef production in 1974 with 720,000 head raised in valley feed lots (down from 798,000 in 1973) with a total value of $\$ 151$ million (down from \$186 million in 1973). While strong in the agricultural sectors, the Imperial County economy is weak in manufacturing and construction activity compared to the state as a whole. Recent county employment patterns show a steady decline in agricultural employment (down $48 \%$ between 1960 and 1970) as agriculture has become more mechanized. During the same time, a steady increase occured in nonagricultural employment. Mexican greencard labor represents under $2 \%$ of the county nonagricultural employment and roughly $70 \%$ of the agricultural work force. Within the county, E1 Centro is the industrial, commercial, and general economic center representing well over half of a11 county retail sales. A second significant center is Calexico, which is located on the U.S.-Mexico border and serves both Imperial. County and Mexican markets.

COUNTY FISCAL CHARACTERIZATION

The Imperial County budget for fiscal year 1976 totaled $\$ 38.7$ million for a per capita budget of $\$ 519.8$. About $25 \%$ of the county's 
revenues that year came from the federal government, and a second $25 \%$ came from the state of California. Income from local taxes and fines accounted for $26.5 \%$ of the county's income. The remainder of fiscal 1976 income was derived Erom carryover and service charges.

During the fiscal year, $\$ 30.8$ million was spent on education by the school districts. The average cost per year per student in an elementary school was $\$ 1,220$, while that of a secondary school student was $\$ 1,385$. These figures have increased over the past decade by $110 \%$ for secondary education and by $170 \%$ for elementary education.

\section{COUNTY SOCIAL CHARACTERIZATION}

The 1974 population of Imperial County is estimated to be just under 84,000; the 1970 official census figure was 74,492. Approximately $95 \%$ of the population is concentrated on the agriculturally rich land of Imperial Valley. The 1970 census reveals major characteristics of this population: an unusually low percentage of young adults (ages 18 to 24); a high percentage of children under 13 yr old; a large number of Mexican-Americans, as would be expected of a border county; the highest percentage of any California county of adults with minimal educational attainment (less than an 8th grade education); and, finally, income levels comparable to most other California counties. GEOTHERMAL IAAUS

Statutes and regulations made at federil, state, and county levels have i strong influence on the development of geothermal resources in the Imperial Valley. The Geothermal Steam Act of 1970 govern: the leasing of federal lands for geothermal projects. Important conditions of the Act are an acreage limitition on leases and the excmption of feteral lands related to the protection of wildife. Regulations promulgated under the legislation are designed to protect the environment as much as possible from the effects of geothermal development. The fideral government also operates a loan guaranty program for geothermal projects. California statures directly associated with geothermal resources control the leasing of state lands and the dril1ing of geothermal wells. Certification of geothermal power-plants is done by the state's Energy Resources Conservation and Development Commission. Imperial County also has regulations that involve geothermal activities. Those regulations ensure that geothermal projects are conducted in a manner that is consistent with existing environmental laws and regulations, county land use zones, and other specific conditions. 


\section{Section 2 \\ Land and Air Resources}

Donald Ermak, Mary Buchanan, and David Layton.

\subsection{GEOGRAPHICAL OVERVIEW}

The Imperial Valley-Salton Sea areas are part of the arid Colorado Desert located in southeastern California (see Fig. 2.1). The Colorado Desert, however, contains on 1 y 2,500 square miles and represents but one of the six major divisions of the vast Sonora Desert that includes southeastern California as we11 as generous portions of Sonora (Mexico), southern Arizona, and northeastern Baja California (Mexico). The combined areas consist of some 120,000 square miles that surround much of the Gulf of California. Although most of the Sonora Desert divisions have much in common, the Colorado Desert is characterized by its lower. inland elevations and an increased water supply from the Colorado River. The north and west boundaries are provided by the Mojave Desert and the Peninsular Range. Included in the Peninsular Range are the San Jacinto and Santa Rosa Mountains in Southern California. The Palo Verde and Coachella Valleys are also part of the Colorado Desert. Except for its Baja California portion, this desert is completely land-locked, separated from the Pacific Coast by a large mountain mass, itself containing several valleys. ${ }^{1}$ At 273 feet below sea level, the Salton Sink in the Imperial Valley is the lowest elevation in the Colorado Desert while in the surrounding mountains, elevations may reach several thousand feet. ${ }^{2}$ Drainage from the low, scattered mountains situated in the eastern portion of the desert flows to the Colorado River and into the Coachella and Imperial Valleys, which have no outlet to the Gulf of California.

In contrast to the arid lands of the Colorado Desert are the verdant agricultural lands of the Imperial Valley. These lands are irrigated with water imported from the Colorado River via the AlI American Canal. Within the irrigated portion of the valley the lands are essentially privately owned; and outside the central part of the valley are federa1 lands managed by the Bureau of Land Management. ${ }^{2,3}$. Wildlife management areas and recreational sites near the Salton Sea constitute other land uses of note. 3 


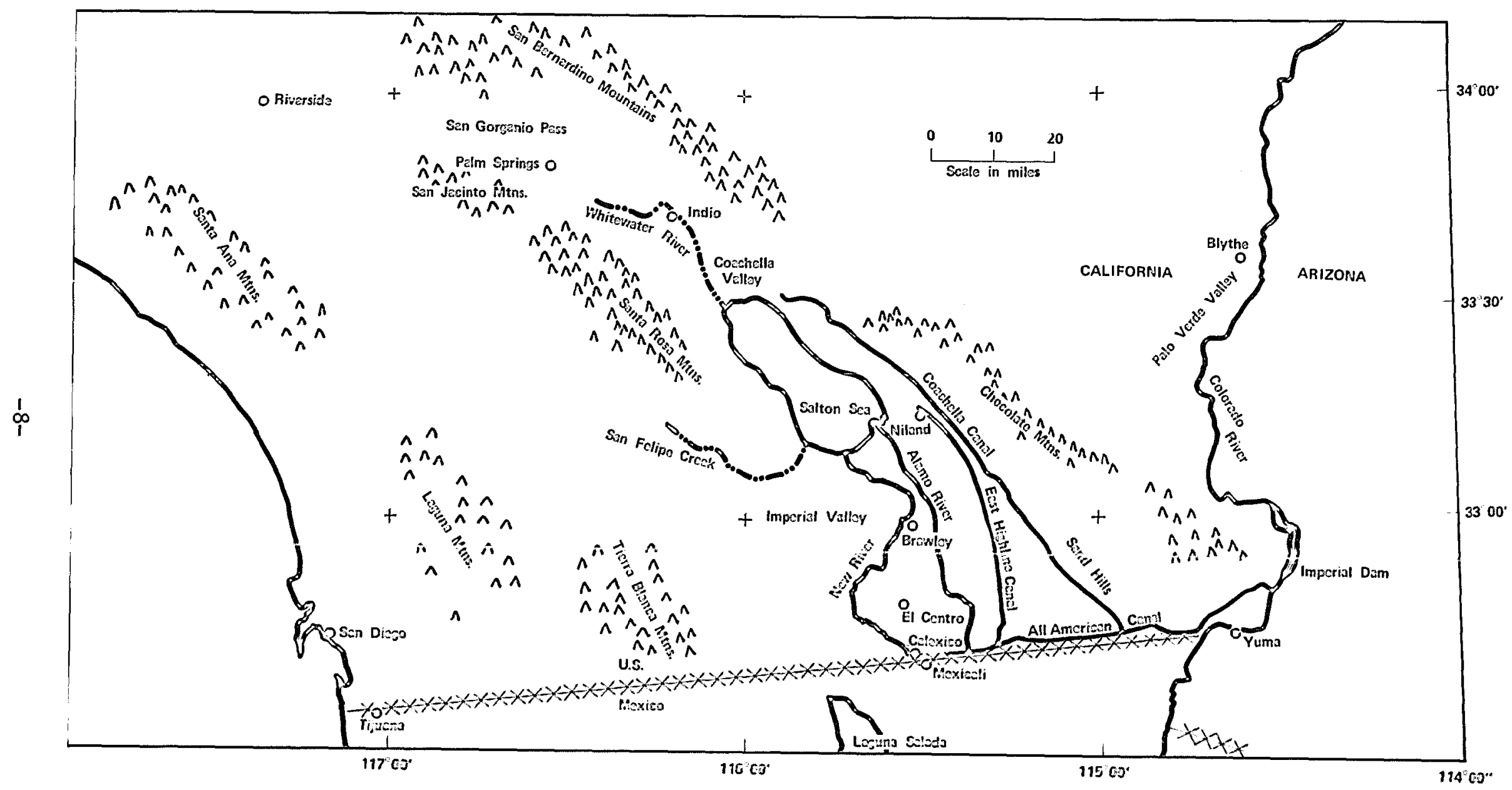

Fig. 2.1. Southeastern California. 


\subsection{GEOLOGY}

The Imperial Valley occupies part of a rift valley known as the Salton Trough. This trough extends from the Gulf of California to the northern segment of the Coachella Valley. It is $200 \mathrm{~km}$ long, between 5 and $130 \mathrm{~km} \mathrm{wide,}{ }^{4}$ and has a maximum sedimentary thickness of about $6 \mathrm{~km}$ above basement rock. 5 Major sedimentary facies include deltaic, lacustrine, alluvial, and aeolian deposits. Current tectonic processes are manifested in the basin by active faults, natura1 subsidence, and earthquakes. Volcanic activity is also associated with parts of the trough.

\section{Sedimentary Rocks}

Sediments in the trough overlay basement rocks consisting of Mesozoic metamorphic rocks that have been intruded by plutonic rocks. 6 Nonmarine deposits from the Colorado River dominate the stratigraphy of the basin, but sediments of marine origin 1ike the Imperial Formation are present as we11. An oil test well drilled to a depth of over 13,000 ft in the central portion of the Imperial Valley indicates that the stratigraphic column is made up of fine grained sandstones and siltstones having chemical characteristics similar to those of the present day Colorado River Delta. 5

Recent lacustrine sediments deposited by prehistoric Lake Cahuilla covers the greatest area in the valley (see Fig. 2.2) and are composed of silts, sands, and clays. The alluvium bordering the mountains that enclose the valley contains silts, sands, and gravels that decrease in size into the finer lacustrine sediments found in the central portion of the valley. 4 Aeolian sands constitute another major facies. The largest deposit in the Salton Trough is known as Sand Hills. The dunes, that exist as parallel waves, are migrating to the southeast under the control of westerly winds. 4

\section{Structure and Tectonics}

A significant feature of the regional geology is the major strike-slip faults exhibiting right lateral movement. Important faults in the Imperial Valley are the San Andreas, Imperia1, San Jacinto, and the Elsinore Faults. 7 One of the more active faults, the Imperial, had almost 2 m lateral displacement during the years 1934 to $1967 .^{8}$ Natural subsidence is occurring in the middle of the valley, while 


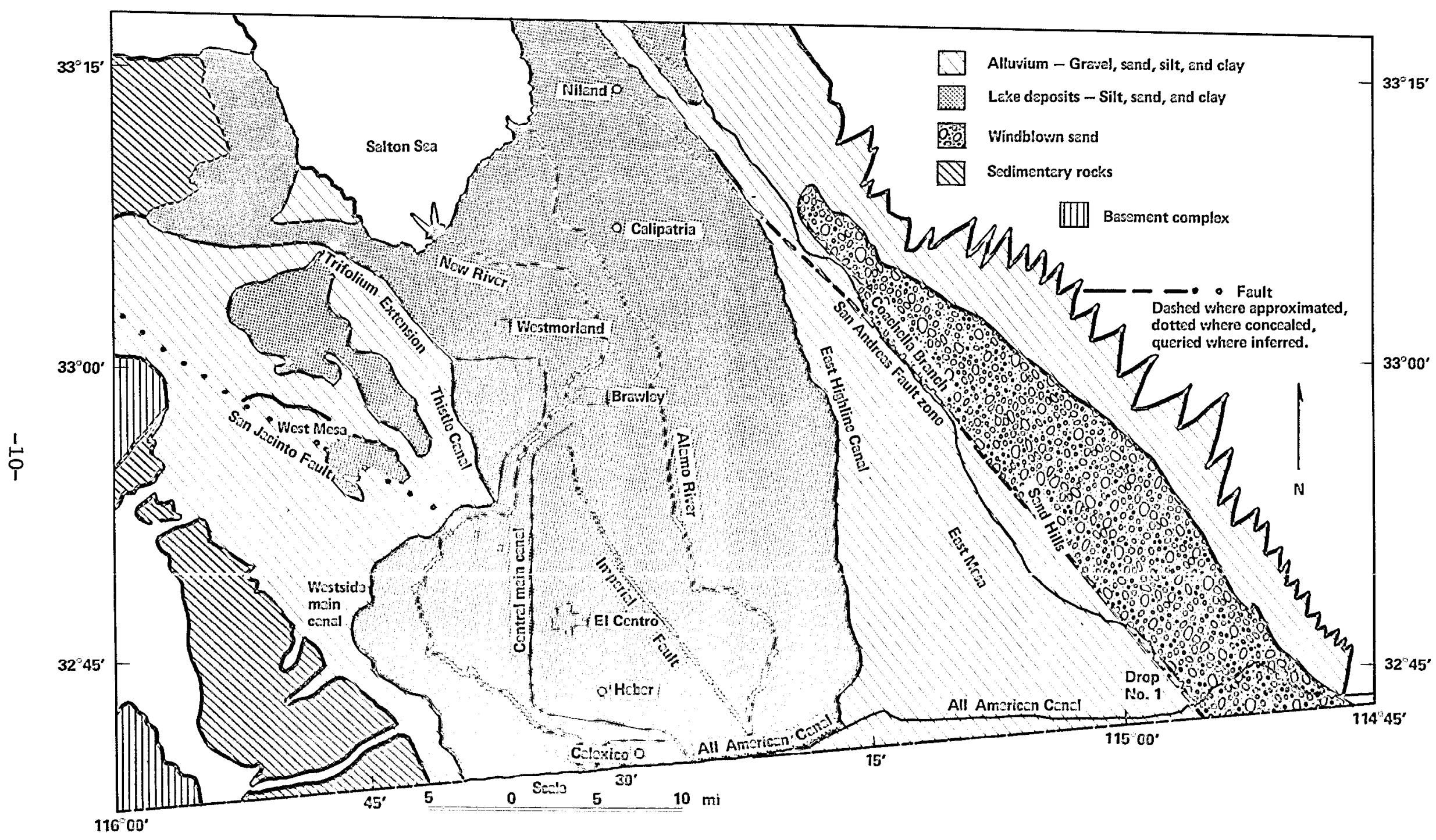

Fig. 2.2. Generalized geologic nap of the Imperial Valley. ${ }^{6}$ 
uplift occurs along the valley sides. Measurements of subsidence taken from 1931 to 1941 revealed downward movements as much as 10 to $20 \mathrm{~cm}^{8}$

Crustal displacements in the valley have been accompanied by a great deal of seismic activity. As many as 12 earthquakes of 6.0 or higher on the Richter scale have hit the Salton Trough in this century. ${ }^{7}$ In addition to the higher magnitude quakes, many earthquakes below a magnitude of 5.0 occur in earthquake swarms. A review of seismic patterns in the Imperial Valley by Hill, et al.${ }^{9}$ revealed the following characteristics:

- A linear alignment of epicenters in the middle of the valley corresponding to the northern part of the Imperial Fault,

- A second concentration of epicenters in the Brawley area,

- An infrequency of earthquakes to the east of the Imperial Fau1t, and

- Depths of most of the quakes between 5 and $14 \mathrm{~km}$. The structural and tectonic characteristics of the Imperial Valley as well as its geothermal anomalies can be explained through plate tectonics and ocean floor spreading. A portion of the earth's crust defined by the Pacific plate is moving in a northwest direction with respect to the American plate. ${ }^{10}$ The main boundary between the two plates is the San Andreas fault zone, part of which passes through the valley. Close to the boundary, earthquakes occur that reflect the crustal movements of the two plates. Spreading centers - areas where the earth's crust is pulling apart forming tension cracks along which lava rises towards the surface - are another possible source of earthquakes. ${ }^{10}$ Moreover, such spreading centers may account for the valley's geothermal anomalies and volcanism. 8,10

\subsection{CLIMATE}

The Imperial Valley has a desert climate with hot, dry summers and mild winters. Data on average temperatures for the last 62 years are displayed in Fig. 2.3. The average temperature in January is about $55^{\circ} \mathrm{F}$, whereas in July it is about $90^{\circ} \mathrm{F}$. This large seasonal temperature difference is indicative of the continental character of the valley's climate. The absence of marine influences is due to high mountain ranges that separate the valley from the California coast. Average diurnal temperature ranges are 20 to $30^{\circ} \mathrm{F}$ throughout the year. Precipitation in the Imperial Valley is very low as shown in Fig. 2.4. The rainy season is from August through March, during which 


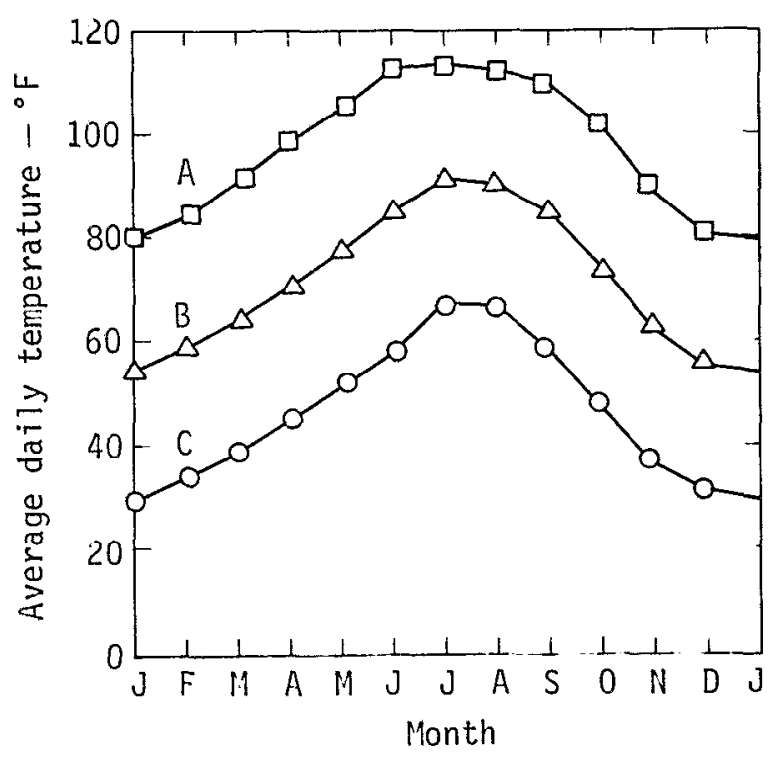

Fig. 2.3. The average daily temperature in Imperial Valley for each month of the year. $A=$ average maximum temperature; $B=$ average temperature; $C=$ average minimum temperature. 11

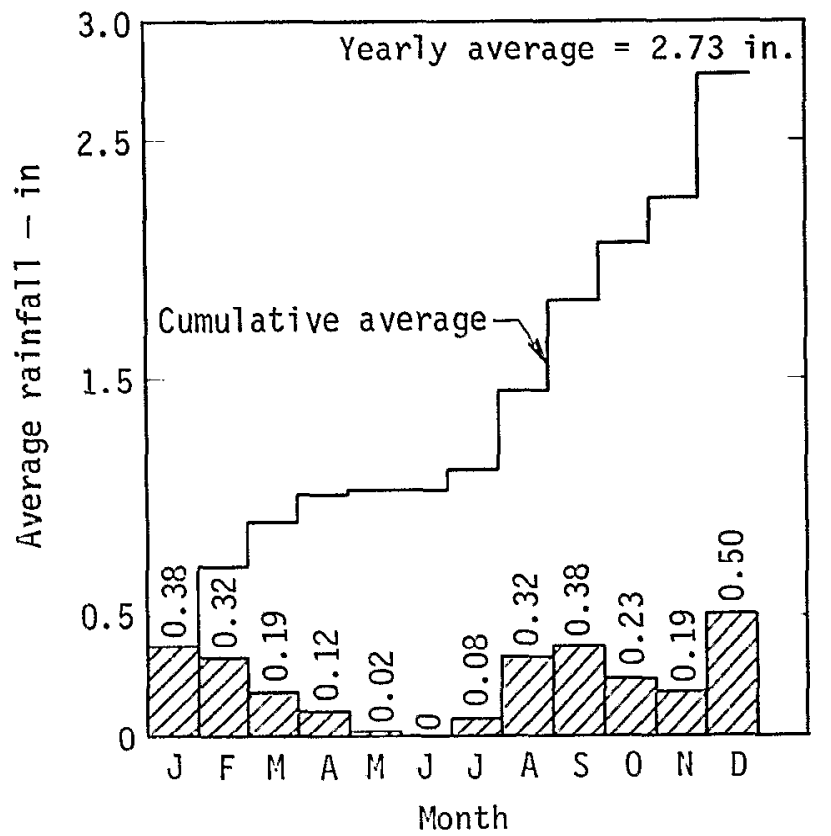

Fig. 2.4. The average monthly rainfall in Imperial Valley. The cumulative average is also shown. 11 there is an average of a little over $3 \mathrm{hr}$ rainfall/mo. June is the driest month with measurable rainfall occurring only once $(0.04 \mathrm{in.on}$ June 2, 1948) since 1914. ${ }^{12}$ The average yearly rainfall is $2.73 \mathrm{in}$. Yearly averages may be misleading, however, as there is considerable variation in rainfall from year to year as shown in Fig. 2.5. Yearly rainfall has varied from a fraction of an inch to over $8 \mathrm{in.}$ The valley has little fog and few thunderstorms. The only recorded snowfall of consequence occurred on December 12, 1932, when $2-1 / 2$ to 4 inches fell throushout the valley. Humidity is very low, especially in the summer. Figure 2.6 shows the averagc rolative humidity for the year 1975 .

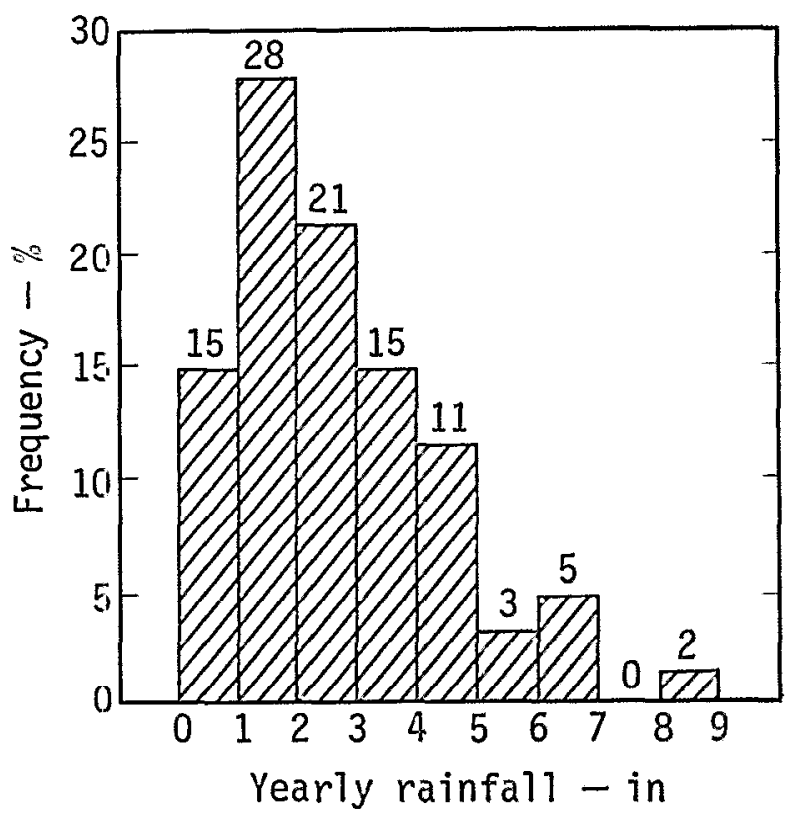

Fig. 2.5. Total yearly rainfall in Imperial Valley. 11 


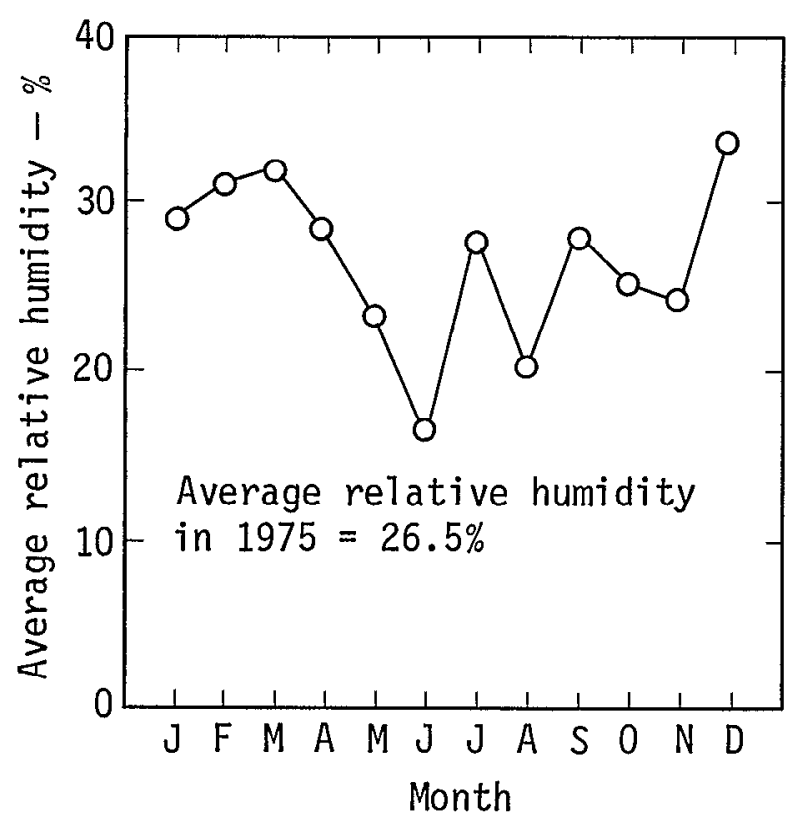

Fig. 2.6. The average relative humidity in the Imperial Valley during 1975.12

Imperial Valley lies in the southeastern part of the California southeast desert basin. A description of the seasonal variations of this region is given by Bennett, ${ }^{13}$ a summary of which follows. In the winter, the basin is generally covered by a moderately intense anticyclonic circulation, except during periods of frontal activity. An average of 20 to 30 frontal systems move into the northern part of the California southeast desert basin each winter. They are relatively weak and become more diffuse as they move southward into the Imperial Valley. Most of the precipitation is associated with these frontal systems. The valley is protected to a large degree from the cold air masses that move southward from Canada over the plains states. This protection, together with the relatively low latitude of the area, results in an average of only 12 frost da/yr.

Spring is a transition period from the winter frontal activity to the dry summer. Temperatures are rising toward the summer highs, and precipitation levels are decreasing toward the low levels of the summer.

During the summer, the Pacific High is well developed to the west of California and a thermal trough overlies the California southeast desert basin. The relative humidity is very low, averaging 30 to $50 \%$ in the early morning and 10 to $20 \%$ in the late afternoon, with humidities of $10 \%$ common during the hottest part of the day. These conditions promote intense heating during the day and marked cooling at night. Temperatures of over $100^{\circ} \mathrm{F}$ typically occur more than 100 days each summer. The intense solar radiation that the valley recelves is highly conducive to photochemical smog formation.

The fall is a transition period back to the frontal activity of the winter. Temperatures are decreasing toward the milder levels experienced in the winter. Precipitation is relatively high with an average of about $0.3 \mathrm{in} / \mathrm{mo}$. 


\subsection{METEOROLOGICAL CONDITIONS}

The dispersion of pollutants emitted into the atmosphere depends upon the winds and the stability of the atmosphere. Pollutant mixing in the direction of the wind is determined by wind speed. Atmospheric stability controls pollutant dispersion in the vertical and horizontal crosswind directions. Stable conditions lead to low dispersion rates, while unstable conditions lead to high dispersion rates. The wind direction determines the locations affected by the polluted air mass.

With hourly measurements taken daily from January 1954 to December 1958 in E1 Centro, the National Oceanic and Atmospheric Administration (NOAA) has calculated the joint frequency distribution for the simultaneous occurrence of a particular wind speed, wind direction, and stability class. ${ }^{14}$ The joint frequency distribution has been calculated on a seasonal and annual basis. Stability class, based on Pasquill's classification system, is a function of net solar radiation and wind speed. ${ }^{15}$ The following discussion summarizes the NOAA results for El Centro.

The frequencies of stability classes are shown in Fig. 2.7 as bar graphs for each season. Classes A through $C$ correspond to unstable conditions, Classes $\mathrm{D}$ and $\mathrm{E}$ to neutral conditions, and class $F$ to stable conditions. Stability class $F$ is the most common condition in the valley, especially in fall and winter.

Figure 2.8 shows the frequency distribution of wind speeds for each of the four seasons. Hind speeds aro divide into 6 groups with the last group being speeds greater than 21 knots $(1 \mathrm{knot}=1.15 \mathrm{mi} / \mathrm{hr})$. The 0 to 3 knot group also shows the frequency of calms, $f_{c}$; a calm is defined as a wind speed less than 1 knot. The yearly average vind speed is about 7.5 knots with the strongest wind conditions occurring during the spring when the average wind speed is about 9 knots. The highest frequency of calms occurs during the winter when calm condition: occur at a frequency of about $9 \%$. Under calm conditions pollutint transfort through the valley is very slow.

Luring most of the year, the prevailing wind direction is predominanty from the west as shown in Fig. 2.9. While some pollutant exchanse does occur between Los Angeles and Riverside counties through the San Corganio pass, the Imperial Valley is essentially shielded from coastal pollutants by the high mountain range:s on the west side of the valley. Durin; the summer, the wind has a 

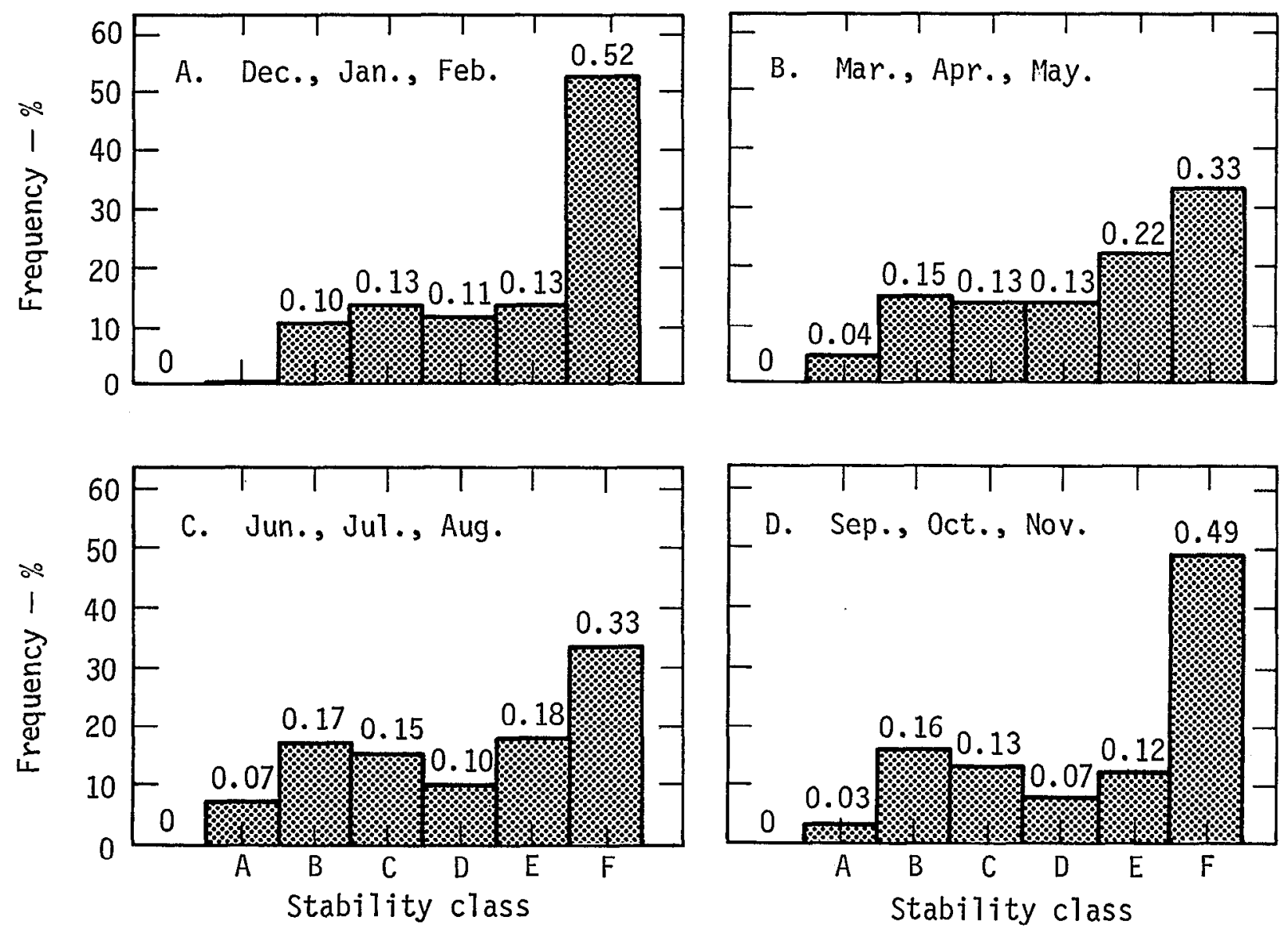

Fig. 2.7. The average atmospheric stability by class in EI Centro. The classification system is that developed by Pasquil1. 15 The frequency distribution is presented for each season of the year.

strong southeasterly component.

Under these conditions, pollutants from the more heavily populated areas in the Mexicali Valley, Mexico, are brought into the Imperial Valley.

An additional influence on the dispersion of pollutants is the occurrence of temperature inversions. When the temperature increases with altitude, the condition is called an inversion. A rising polluted air mass that encounters a sufficiently strong inversion layer is prevented from further upward motion. Vertical mixing is then confined to the layer of air beneath the inversion. These conditions are prevalent at night throughout the year when mixing in the lower atmosphere is limited to a height of 200 to $2000 \mathrm{ft} .^{13}$ During the summer, inversions are destroyed early in the day by intense solar heating, but persist throughout much of the day in the winter. 

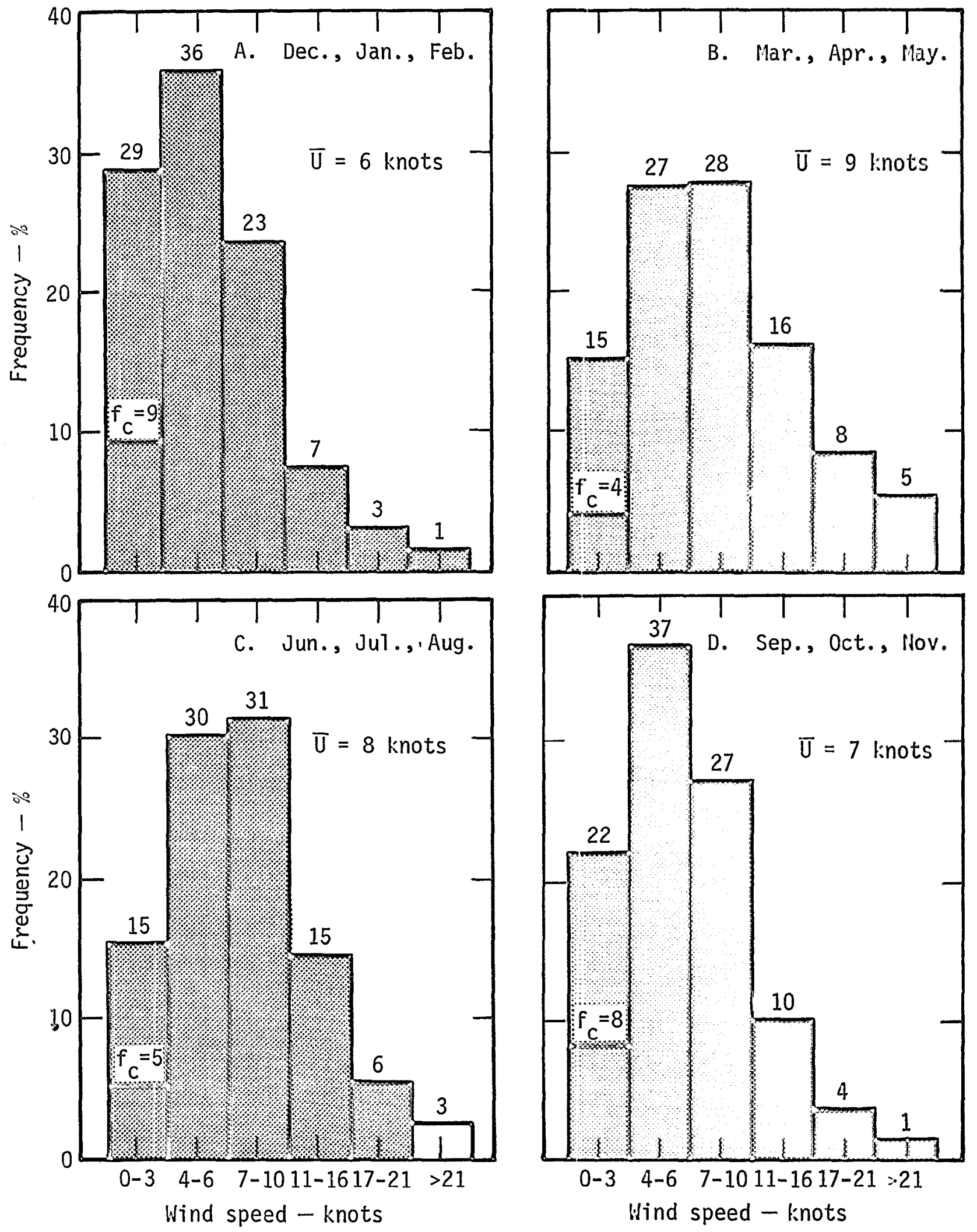

Fig. 2.8. The wind speeds at E1 Centro. The frequency distribution is presented for each season of the year. $\bar{U}=$ average wind speed, $\mathrm{f}_{c}=$ frequency of calms. 
A. Dec., Jan., Feb.

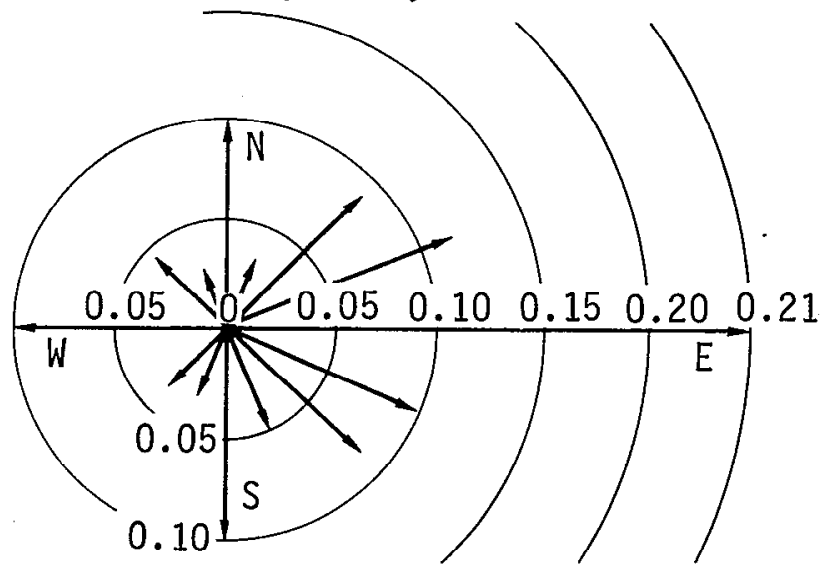

C. Jun., Jut., Aug.

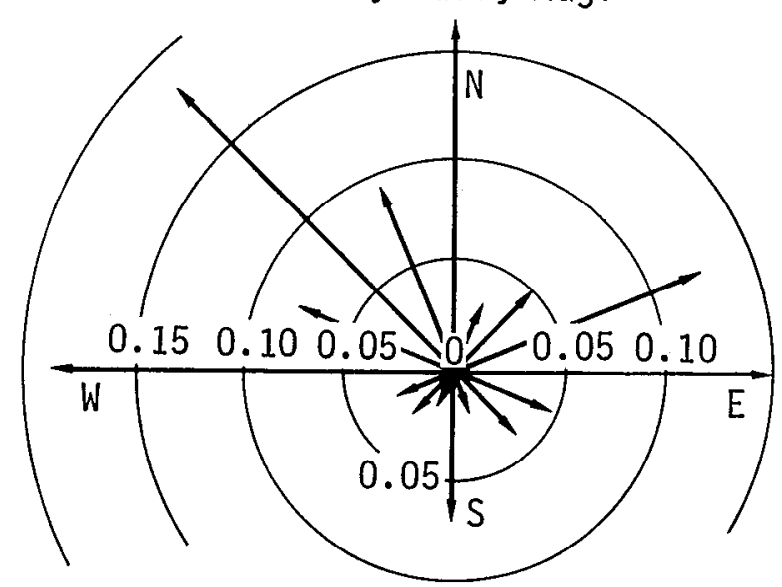

B. Mar., Apr., May.

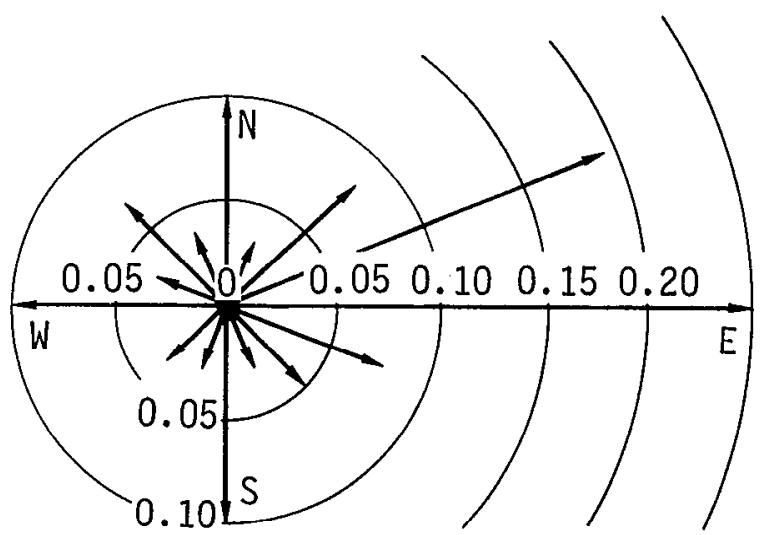

D. Sep., Oct., Nov.

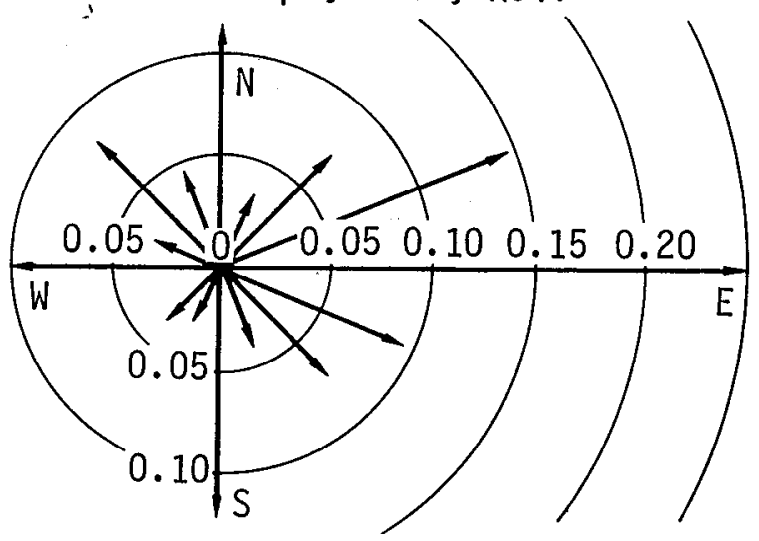

Fig. 2.9. Polar plots of the frequency distribution of wind direction at

El Centro. The arrows indicate the direction from which the wind is blowing and their lengths represent the frequency of occurrence in percent.

\subsection{AIR QUALITY}

Stations recording air pollution concentrations are located in Brawley, Calexico, and E1 Centro. Sulfur dioxide $\left(\mathrm{SO}_{2}\right)$, ozone, particulates, and lead are measured at EI Centro; particulates are measured at Brawley and Calexico. The nearest station measuring additional pollutants is at Indio, which is to the north of the Salton Sea. Figure 2.10 shows the monthly maximum hourly concentrations of nitric oxide (NO), nitrogen dioxide $\left(\mathrm{NO}_{2}\right)$, carbon monoxide $(\mathrm{CO})$, and oxidants at the Indio station from June 1974 to May 1975. The levels of oxidant are greatest during the months of high solar radiation when the most photochemical activity occurs. Peak concentrations of CO and No in the winter months probably reflect increased vehicular emissions associated with agricultural production and tourism. Space heating may also contribute to those concentrations. 


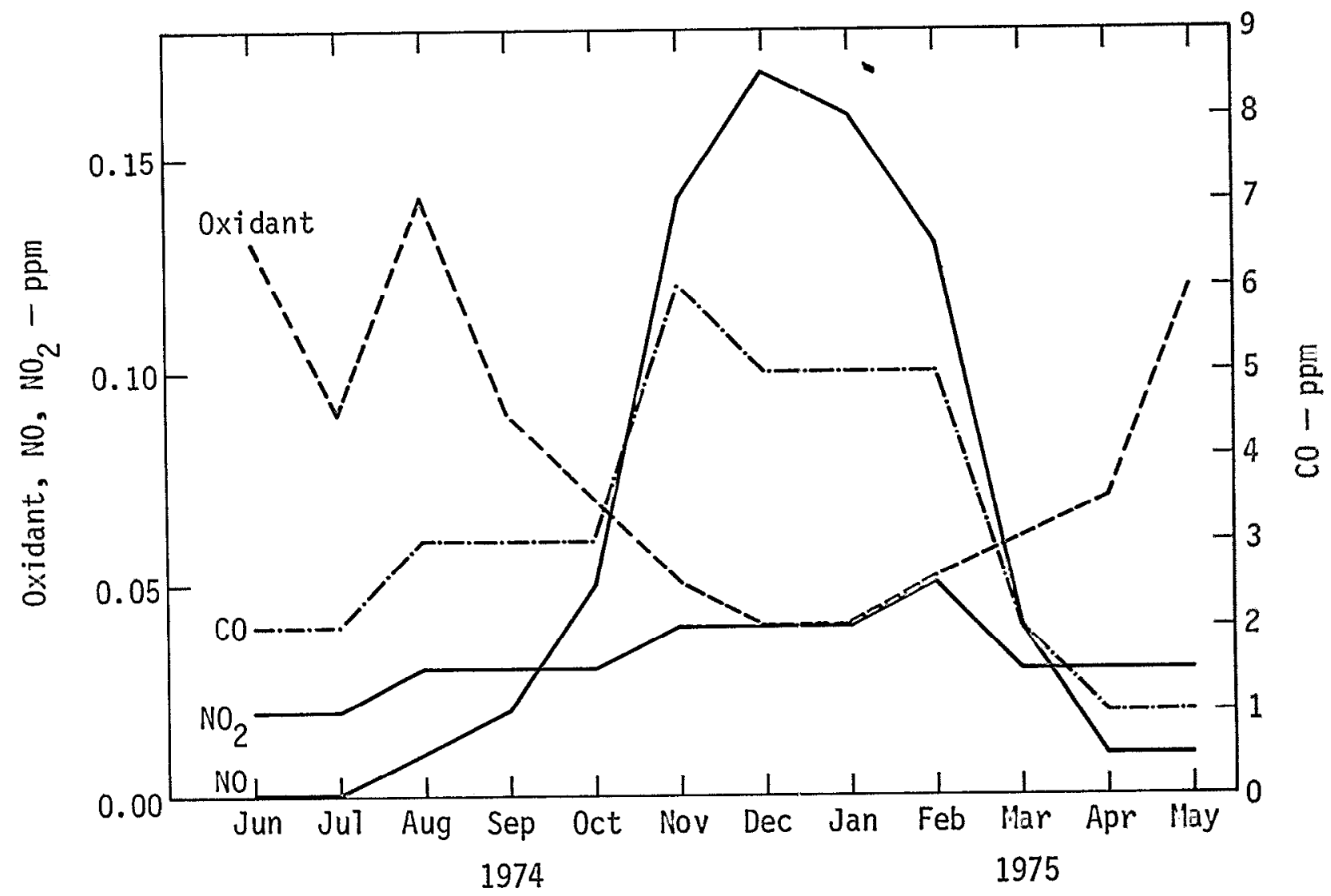

Fig. 2.10. Monthly maximum averages of oxidant, $\mathrm{NO}, \mathrm{HO}_{2}$, and $\mathrm{CO}$ at Indio, California. 16

Air pollutants monitored in the Imperial Valley exhibit seasonal patterns similar to those at Indio. For cxample, the monthly maximum hourly averages of ozone, a photochemical oxidant, decrease in the winter at El Centro (Fig. 2.11) as do the oxidant levels measured at Indio. Atmospheric lead, which is derived mainly from motor vehicles, rises in the winter months (Fig. 2.12) when there is more traffic in the valley. The same increase is seen in NO and Co levels at Indio.

Month1y maximum hourly averages of $\mathrm{SO}_{2}$ (Fig. 2.11) remain almost constant throughout the year. The absence of major industrial sources of $\mathrm{SO}_{2}$ accounts for its static nature. Particulate concentrations, in contrast, display a lot of variability. Wonthly mean particulate concentrations at EI Centro and Calexico (Fig. 2.13) reveal temporal differences that are probably related to the type of ground cover in the vicinity of the cities as well as to localized meteorological conditions. The relatively high level of particulate mass loading at these stations seems: to represent a regional phenomenon since Bravley, located 


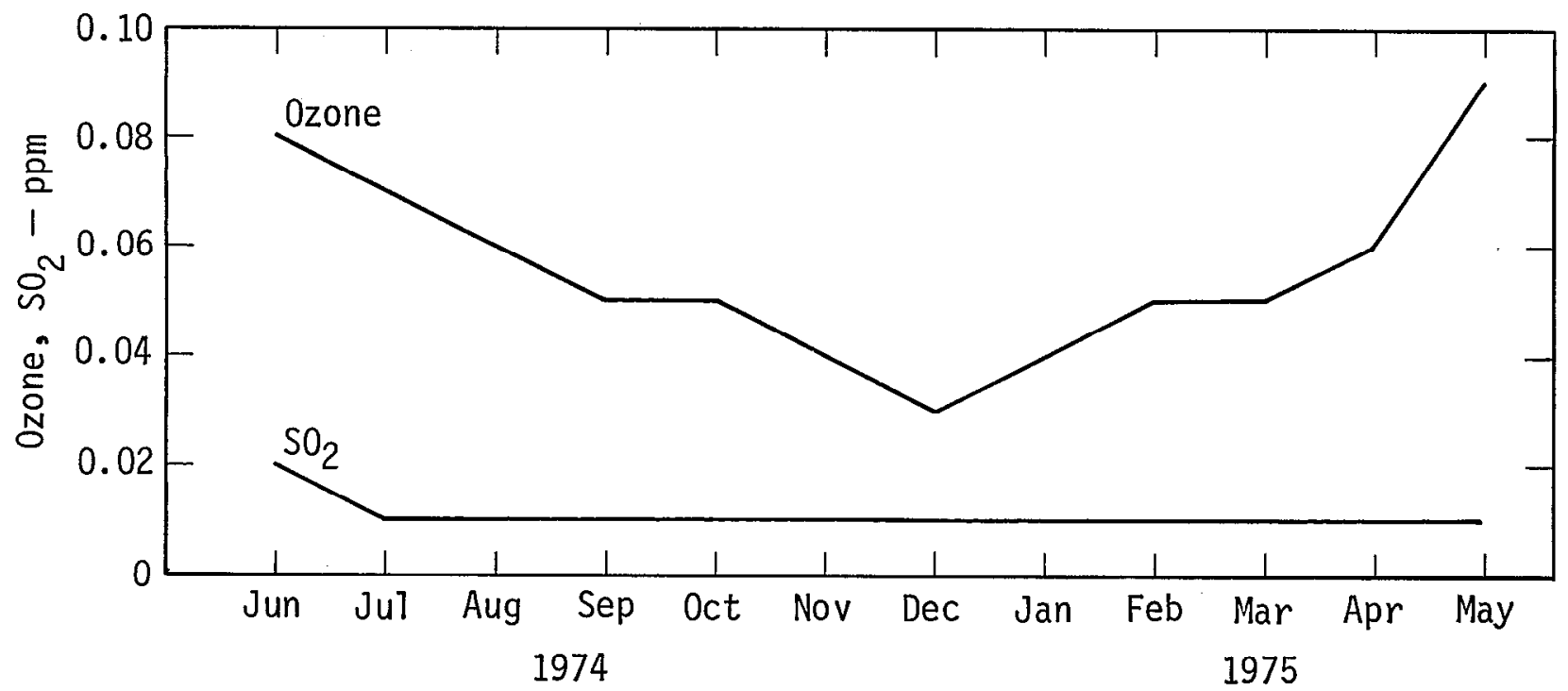
Fig. 2.11. Monthly maximum hourly averages of ozone and $\mathrm{SO}_{2}$ at El Centro,
California. 16

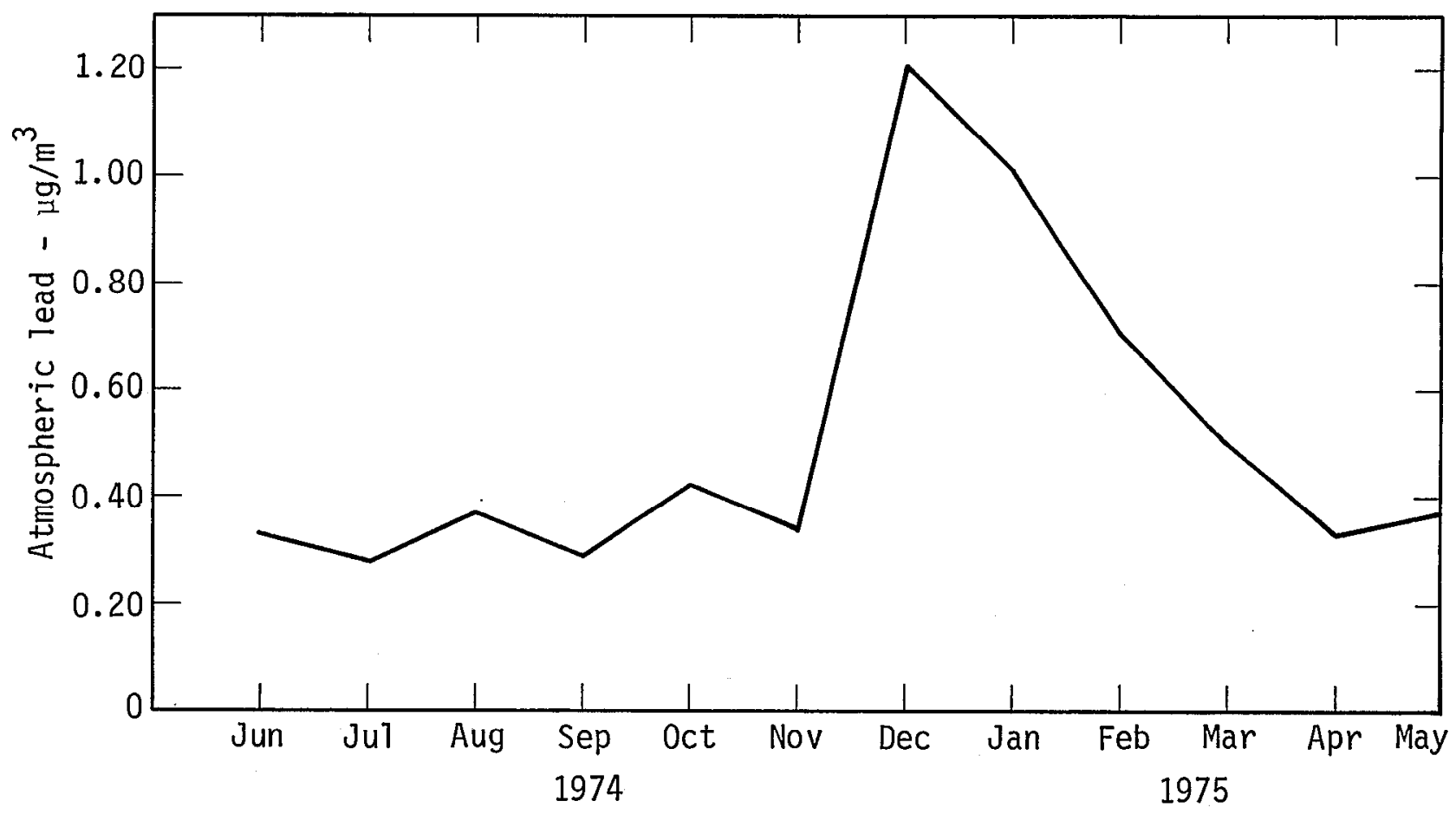

Fig. 2.12. Monthly averages of atmospheric lead at E1 Centro, California. ${ }^{16}$ 


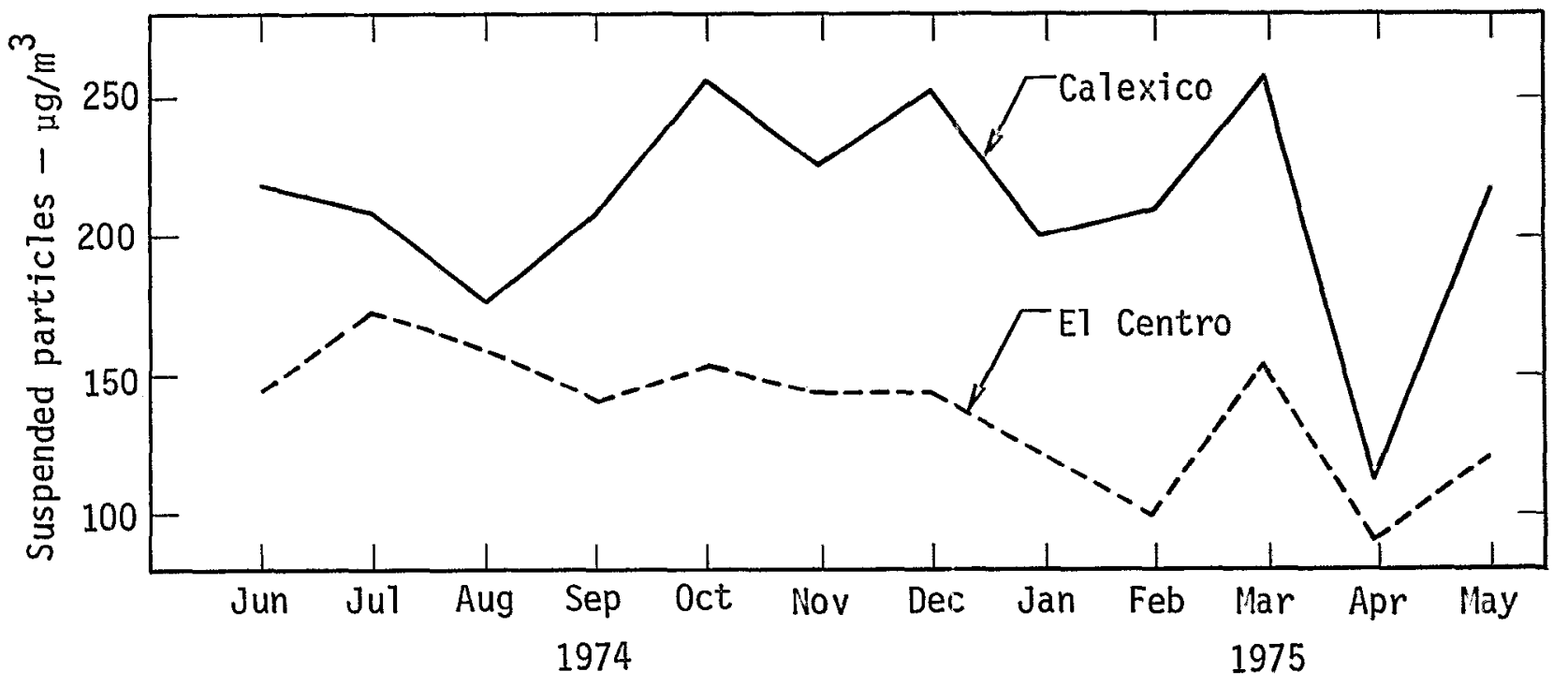

Total suspended particulates

Fig. 2.13. Total suspended particulates. Mean of 24-hr observations. ${ }^{16}$

near the middle of the valley, also records high particulate levels. In fact, during a 12-month period (June 1974 to May 1975) the geometric mean of particle concentrations measured at the Brawley stations was $211 \mu \mathrm{g} / \mathrm{m}^{3}$, higher than both the annual primary and secondary national standards (i.e., $75 \mu \mathrm{g} / \mathrm{m}^{3}$ and $\left.60 \mu \mathrm{g} / \mathrm{m}^{3}\right)$.

\section{REFERENCES}

1. R. M. Ryan, Mommals of Deep Canyon, The Desərt Museum, Palm Springs, California (1968).

2. Report of the Status of Development of Geothermal Energy Recourees in California, Jet Propulsion Laboratory, California Institute of Technology (March 31, 1976).

3. U.S. Department of the Interior and the Resources Agency of California, Salton Sea Project, California, Federal-State Reconnaissance Report (1969).

4. P. C. Van De Kamp, "Holocene Continental Sedimentation in the Salton Basin, California: A Reconnaissance," Geo. Soc. Am. BulZ. 84 (3), 827 (1973).

5. P. Muffler and B. R. Doe, "Composition and Mean Age of Detritus of the Colorado River Delta in the Salton Trough, Southeastern California," J. Sediment. Petrol. 38 (2), 384 (1963). 
6. 0. J. Loeltz, B. Ireland, J. H. Robison, and F. H. Olmsted, Geohydrologic Reconnaissance of the Imperial Valley, California, Geological survey Professional Paper 486-K, U.S. Government Printing Office, Washington, D.C. (1975).

7. T. D. Palmer, J. H. Howard, and D. P. Lande, Geothermal Development of the Salton Trough, California and Mexico, Lawrence Livermore Laboratory, Rept. UCRL-51775 (1975).

8. W. A. Elders, R. W. Rex, T. Meidav, P. T. Robinson, and S. Biehler, "Crustal Spreading in Southern California," Science 178, 15 (1972).

9. D. P. Hill, P. Mowinckel, and L. G. Peake, "Earthquakes, Active Faults, and Geothermal Areas in the Imperial Valley, California," Science 188, 1306 (1975).

10. H. W. Menard, Geology, Resources, and Society, (W. H. Freeman and Company, San Francisco, 1974).

11. Imperial Irrigation District, Imperial Irrigation District, Water Report, 1974, unpublished.

12. Imperial Irrigation District, Water Master, Tmperial ValZey 1975 Weather Summary (1975).

13. C. I. Bennett, California Air Resources Board, Climate of the Southeastern Desert Air Basins 1975.

14. National Oceanic and Atmospheric Administration, Star Program Data, Environmental Data Science, Ashville, N. Carolina.

15. F. Pasqui11, "The Estimation of Dispersion of Windborne Material," Meteorological Magazine, 90, 33 (1961).

16. California Air Resources Board, California Air Quality Data, Vol. 6, No. 3 (1974) through Vol. 7, No. 3 (1975). 


\section{Section 3 \\ Water Resources}

David Layton

About 3 million acre-feet (af) of Colorado River water are diverted to the Imperial Valley each year to support irrigated agriculture. Over 1,700 miles of main canals and laterals distribute water to a service area encompassing 501, 264 acres of which about 475,000 acres are irrigated. Another 1,400 miles of drainage ditches carry waste waters to the Salton Sea. Underlying the valley are shallow aquifers exhibiting both artesian and water table conditions. The deeper aquifers contain large amounts of water, some of which is geothermally heated to temperatures greater than $300^{\circ} \mathrm{C}$. Surface waters range from about 900 ppm total dissolved solids (TDS) below Drop 1 on the All American Canal (see Fig. 3.1) to over $39,000 \mathrm{ppm}$ in the Salton Sea; ground water salinities range from a few hundred ppm in East Mesa to over $10,000 \mathrm{ppm}$ in scattered wells situated in the middle of the valley.

\subsection{GROUND WATER}

Information on the hydrologic properties of the valley's waterbearing sediments is largely restricted to shallow aquifers where pump tests have been conducted. Those tests show that the most productive aquifers are found on the eart and west sides of the valley. Transmissivities computed from pump tests vary from 37,000 to about 300,000 gal/da-ft on the western side of the valley; 47,000 to over $800,000 \mathrm{gal} / \mathrm{da}-\mathrm{ft}$ in the East Mesa - Sand Hills area. The probable range of transmissivities for the central valley is from 1,000 to $10,000 \mathrm{gal} / \mathrm{da}-\mathrm{ft}$ at depths to $500 \mathrm{ft}$. Transmissivities are greatest on the eastern and western sides of the valley because the alluvial deposit: in those areas have higher permeabilities than the finer grained silts and clays of the valley floor.

The main source of recharge to aquifers is the unlined canals that distribute irrigation water to the Coachella and Imperial Valleys. From 1950 to 1967 the calculated cumulative leakage along the Al1 American Canal from Pilot Knob to the East Highline Canal was approximately 4.5 million af. $^{1}$ Leakage during the same period along the Coachella Canal (from the All American Canal to a point paralle1 with Niland) was estimated to be 2.7 million af. In comparison to this artificial recharge, natural recharge to the valley from the Colorado River is estimated at just 17,000 af 
annual1y. $^{1,2}$ Some recharge is also associated with deep percolation of irrigation water; however, an extensive subsurface drainage system removes most of that water to the Salton Sea.

Water is discharged from the sha1low aquifers to springs, wells, rivers, agricultural drains, and the Salton Sea. Springs yield a few thousand acre-feet each year as do flowing wells in an area between the East Highline Canal and the Alamo River. Annual discharge from the alluvial aquifers bordering the southern portion of the Salton Sea is probably more than 10,000 af. ${ }^{3}$ An additional 10,000 to 20,000 af discharges to rivers and drains. ${ }^{1}$

The movement of ground water in the valley is shown by the waterlevel contour map in Fig. 3.1. Recharge areas are located near San Felipe Creek, East Mesa, Niland, and West Mesa. Ground water discharge occurs along the lower reaches of the New and Alamo Rivers, as indicated by the bending of the contours upstream as they cross the rivers.

The amount of water held in storage by the valley's aquifers is enormous. Dutcher et a1. ${ }^{2}$ estimate that 1.1 billion af of recoverable water is contained in water bearing sediments, and their estimate does not even include geothermal brines having salinities in excess of 35,000 ppm TDS. Nearly a third of the recoverable water (349 million af) resides in a vertical zone defined by Dutcher et al. ${ }^{2}$ as extending from the water table to a surface limited by either bedrock, the $100^{\circ} \mathrm{C}$ isotherm, or a depth of 3,000 feet, whichever is shallowest. Aquifers underlying the Sand Hills, East Mesa, and West Mesa portions of the valley that are in this zone have specific yields (i.e., the volume of water a saturated sediment will yield to gravity divided by the sediment's total volume, expressed as a percent) ranging from 15 to $20 \%$.

\subsection{GROUND WATER QUALITY}

In general, the ground water quality of the shallow aquifers is best on the eastern and western sides of the valley. Water of lesser quality is found in both the central and western parts of the valley. According to U.S. Geological Survey Professional Paper $486-\mathrm{K},{ }^{1}$ which has water quality data from the late $1950^{\prime}$ 's and early 1960 's, wells on East mesa yielded water with TDS's ranging from over 500 ppm to 7,280 ppm TDS. Most of those wells, however, had water less than 1,000 ppm TDS. Ground waters in the central valley, on the other hand, were almost all between $1,000 \mathrm{ppm}$ to 3,000 ppm TDS. The highest TDS in a well was $15,700 \mathrm{ppm}$. In the western section of the valley, water varied 


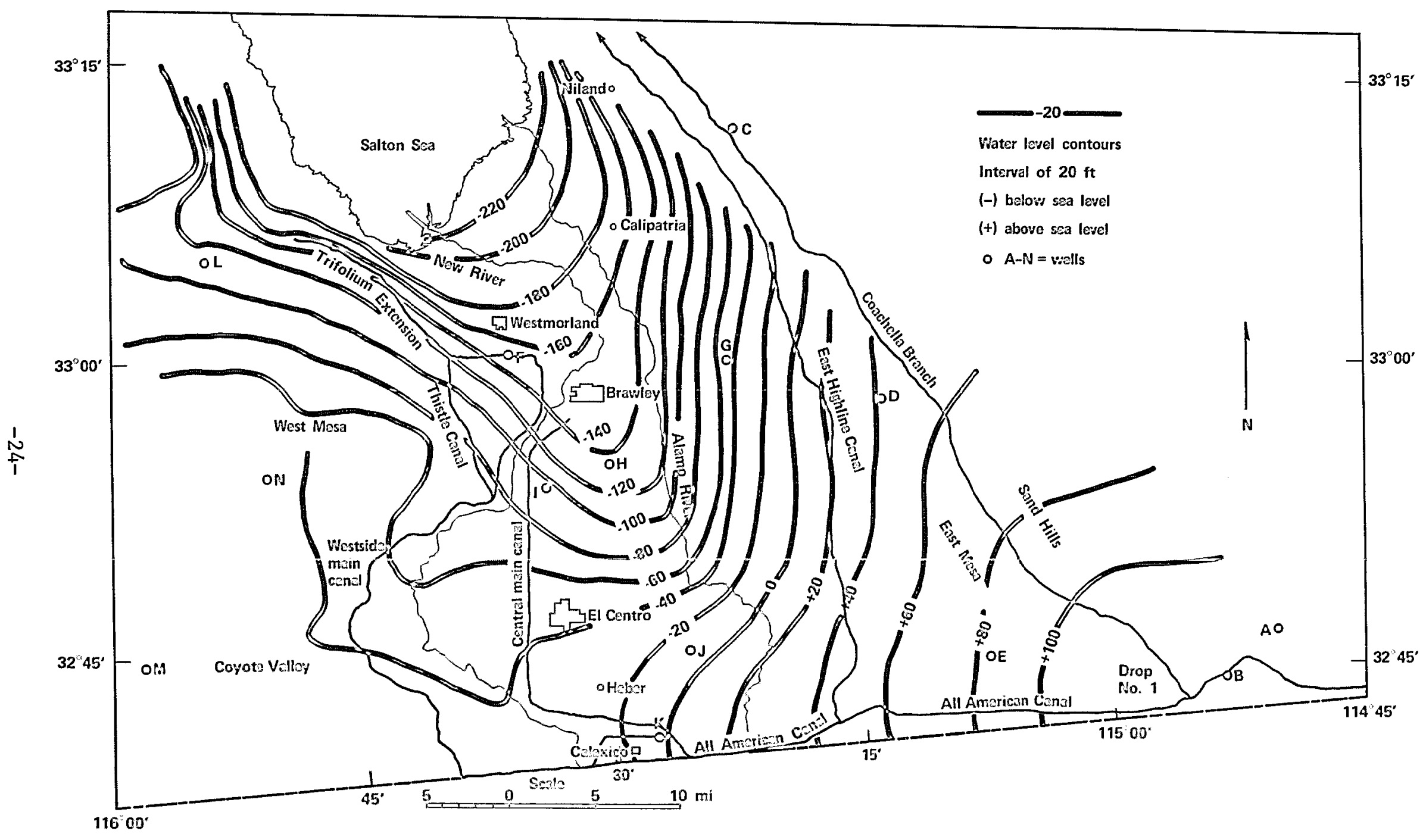

Fig. 3.1. Water-level altitudes in 1965 for Inperial valley. ${ }^{1}$ 
widely in quality: almost all of Coyote Valley's wells (see Fig. 3.1) had water below $500 \mathrm{ppm}$, while West Mesa wells had water between 1,800 ppm and 5,200 ppm. Table 3.1 gives chemical analyses of selected wells in the Imperial Valley.

\subsection{SURFACE WATER}

Surface waters in the valley are derived from the Colorado River at Imperial Dam. Water imported by the Coachella Valley County Water District (CVCWD) and the Imperial Irrigation District (IID) is part of California's share of the Colorado River. In addition, Mexicali Valley receives water from the Colorado River, some of which drains across the border in the New and Alamo Rivers. Because of soil salinity problems, an extensive subsurface drainage system has evolved since 1929 that removes salts leached through soils underlying more than 388,000 acres. Drain water resulting from leaching as well as direct runoff from irrigated fields is carried by drainage structures to the New River, Alamo River, and the Salton Sea.

Imported Colorado River water or drainage waters are possible sources of cooling water for geothermal power plants. The availability of those waters for cooling - irrespective of institutional and legal considerations is presently dependent on irrigation practices and cropping trends. Historic the canals, which is being reduced flows into the valley measured at Drop No. 1 on the All American Canal have averaged below 3 million af since 1951 (Fig. 3.2); the corresponding waste water flows to the Salton Sea average between 35 and $40 \%$ of the in-flows at Drop No. 1.

\section{Daily diversions to Imperial} Valley from the Imperial Dam are based on requests submitted to the U.S. Bureau of Reclamation by the IID. Those requests are made one week in advance of the actual diversions and are based on the anticlpated water use in the valley. The demands for water follow planting and harvesting schedules, peaking in the months of April, July, August, and September when crops are planted. 4,5 Water destined for the valley is released at Parker Dam, flows 3 days to reach the Imperial diversion dam, then takes another day in transit to the Imperial Valley. 6

When the water arrives in the canals of the irrigation district, its distribution is governed by water orders submitted to the IID by the valley's irrigators 3 days prior to delivery. 7 The ability of the irrigation system to meet water requests for a given day depends on the amount of water already stored in the district's canals plus the inflow from the All American Canal. Other than the storage capacity of 
Table 3.1. Water quality data for selected wells in the Imperial Valley. ${ }^{1}$

\begin{tabular}{|c|c|c|c|c|c|c|c|c|c|c|c|c|c|c|c|}
\hline $\begin{array}{l}\text { Well } \\
\text { identifi- } \\
\text { cation }^{2}\end{array}$ & $\begin{array}{c}\text { Interval } \\
\text { sampled } \\
\text { (ft below } \\
\text { land } \\
\text { surface) }\end{array}$ & $\begin{array}{l}\text { Year } \\
(19)\end{array}$ & $\begin{array}{l}\text { Silica } \\
\left(\mathrm{SiO}_{2}\right)\end{array}$ & $\begin{array}{l}\text { Calcium } \\
\text { (Ca) }\end{array}$ & $\begin{array}{l}\text { Magne- } \\
\text { siumi } \\
\text { (Mg) }\end{array}$ & $\begin{array}{l}\text { Sodium and } \\
\text { potassium }\end{array}$ & $\begin{array}{l}\text { Eicar- } \\
\text { bonate } \\
\left(\mathrm{HCO}_{3}\right)\end{array}$ & $\begin{array}{l}\text { Sul- } \\
\text { fate } \\
\left(\mathrm{SO}_{4}\right)\end{array}$ & $\begin{array}{l}\text { Chlo- } \\
\text { ride } \\
\text { (C1) }\end{array}$ & $\begin{array}{l}\text { Fluoride } \\
\text { (F) }\end{array}$ & $\begin{array}{l}\text { Tota1 } \\
\text { dis- } \\
\text { solved } \\
\text { solids }\end{array}$ & $\begin{array}{l}\text { Hardness } \\
\text { Calcium } \\
\text { magnesium }\end{array}$ & $\begin{array}{l}\mathrm{CaCO}_{3} \\
\text { Non- } \\
\text { carbon- } \\
\text { ate }\end{array}$ & $\begin{array}{l}\text { Percent } \\
\text { sodium }\end{array}$ & $\mathrm{pH}$ \\
\hline A & $150-152$ & 62 & 4 & 40 & 10 & $\begin{array}{c}(\text { in } \mathrm{mg} / 1 .) \\
301\end{array}$ & 116 & 135 & 397 & -- & 945 & 142 & 47 & 82 & 6.9 \\
\hline B & $127-144$ & 63 & 15 & 95 & 32 & 126 & 174 & 317 & 119 & .3 & 791 & 368 & 226 & 43 & 7.5 \\
\hline $\mathrm{C}$ & $25-150$ & 63 & 33 & 106 & 107 & 503 & 212 & 700 & 635 & 1.6 & 2,190 & 705 & 531 & 61 & 7.4 \\
\hline D & $113-115$ & 62 & 27 & 88 & 45 & 578 & 147 & 308 & 865 & - & 1,980 & 405 & 284 & 76 & 8.1 \\
\hline E & $155-157$ & 64 & 22 & 26 & 11 & 280 & 150 & 212 & 265 & 1.4 & 892 & 112 & 0 & 84 & 8.0 \\
\hline $\mathrm{F}$ & $145-147$ & 62 & 16 & 564 & 460 & 3,100 & 434 & 1,250 & 5,950 & - & 11,600 & 3,300 & 2,940 & 67 & 7.2 \\
\hline G & -- & 62 & 18 & 31 & 8.6 & 952 & 424 & 525 & 915 & - & 2,660 & 113 & 0 & 95 & 8.1 \\
\hline H & $82-84$ & 62 & 16 & 1,610 & 1,110 & 1,770 & 352 & 2,050 & 7,100 & -- & 13,800 & 8,580 & 8,290 & 31 & 7.2 \\
\hline I & $124-126$ & 62 & 25 & 676 & 417 & 3,930 & 416 & 875 & 7,580 & -- & 13,700 & 3,400 & 3,406 & 72 & 7.4 \\
\hline $\mathrm{J}$ & $145-147$ & 62 & 14 & 376 & 214 & 2,920 & 267 & 400 & 5,350 & -- & 9,410 & 1,820 & 1,600 & 78 & 7.4 \\
\hline $\mathrm{n}$ & $150-152$ & 62 & 11 & $2 /: 4$ & 161 & 1.530 & 25.7 & $85 n$ & $2,49 n$ & - & 5,410 & 1.270 & 1,060 & 72 & -- \\
\hline $\mathrm{L}$ & -- & 62 & 0 & 109 & 46 & 1,590 & 66 & 5 & 2,740 & -- & 4,520 & 462 & 408 & 88 & 6.6 \\
\hline $\mathrm{M}$ & -- & 62 & 20 & 25 & 7.4 & 108 & 156 & 43 & 109 & 1,0 & 391 & 93 & 0 & 72 & 7.9 \\
\hline N & $135-560$ & 62 & 15 & 152 & 33 & 510 & 64 & 1,080 & 318 & -- & 2,140 & 515 & 463 & 68 & 7.7 \\
\hline
\end{tabular}

\footnotetext{
a See Figure 3.1 for lacition.
} 


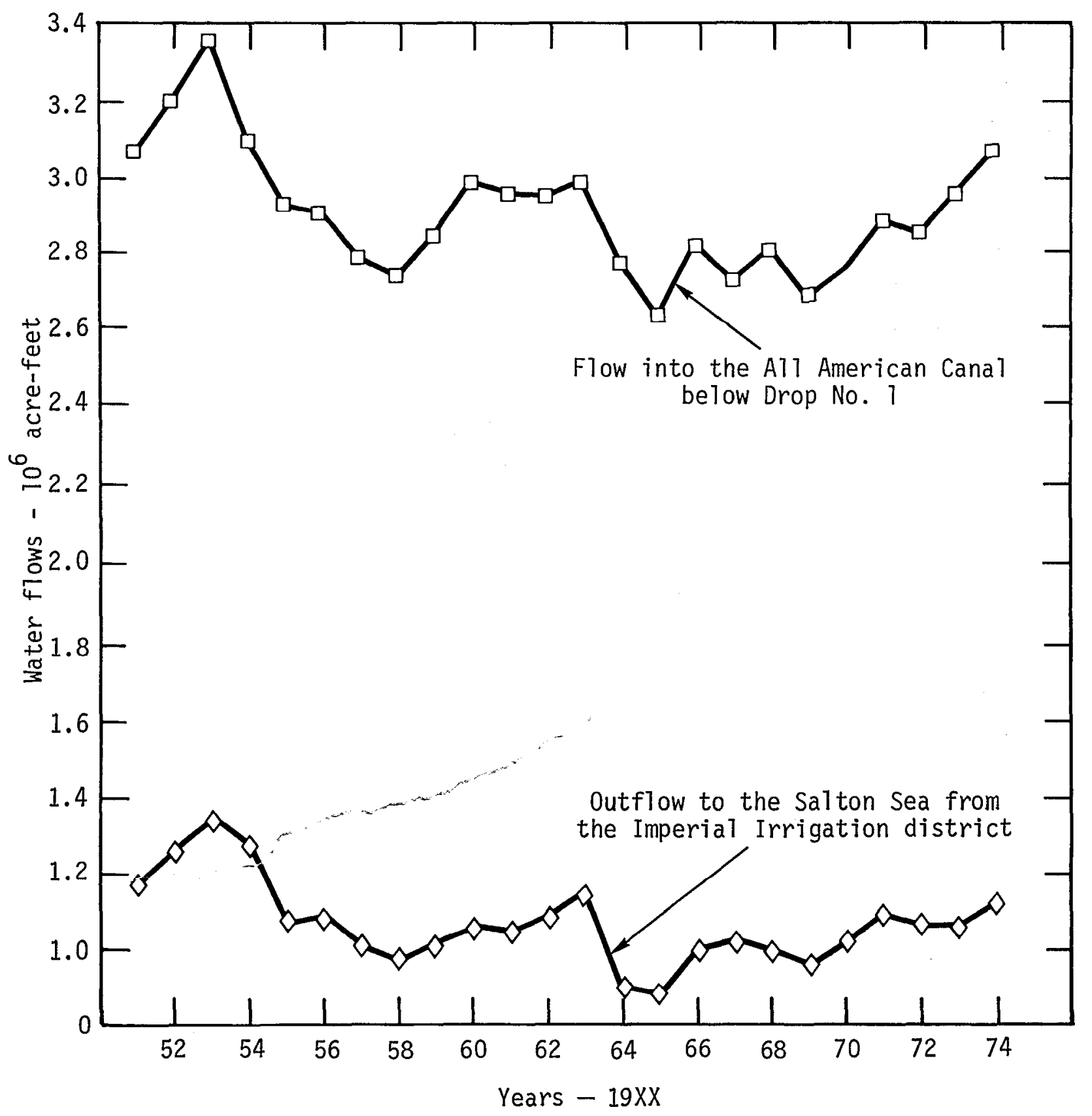

Fig. 3.2. Inflows and outflows of the Imperial Irrigation District during $1951-1974.5$

by concrete lining, there is only one regulatory pond within the irrigation system to provide hold-over storage for periods when a surplus of water occurs.
The Senator Wash facility on the Colorado River can also be used for temporary storage by IID during times of excess water in the canals. 


\subsection{SURFACE WATER QUALITY}

The quality of surface water in

the valley depends primarily upon

the chemical, physical, and biological characteristics of Colorado River water; New River inflows from Mexico; and effluent flows from the irrigation system. of particular concern within recent years has been the deteriorating quality of Colorado River water diverted to the valley. Figure 3.3 shows a distinct rising trend in the river's salinity, which now stands at about $850 \mathrm{ppm}$ TDS. Predictions of salinity ${ }^{8,9}$ indicate continued increases that, even with salinity

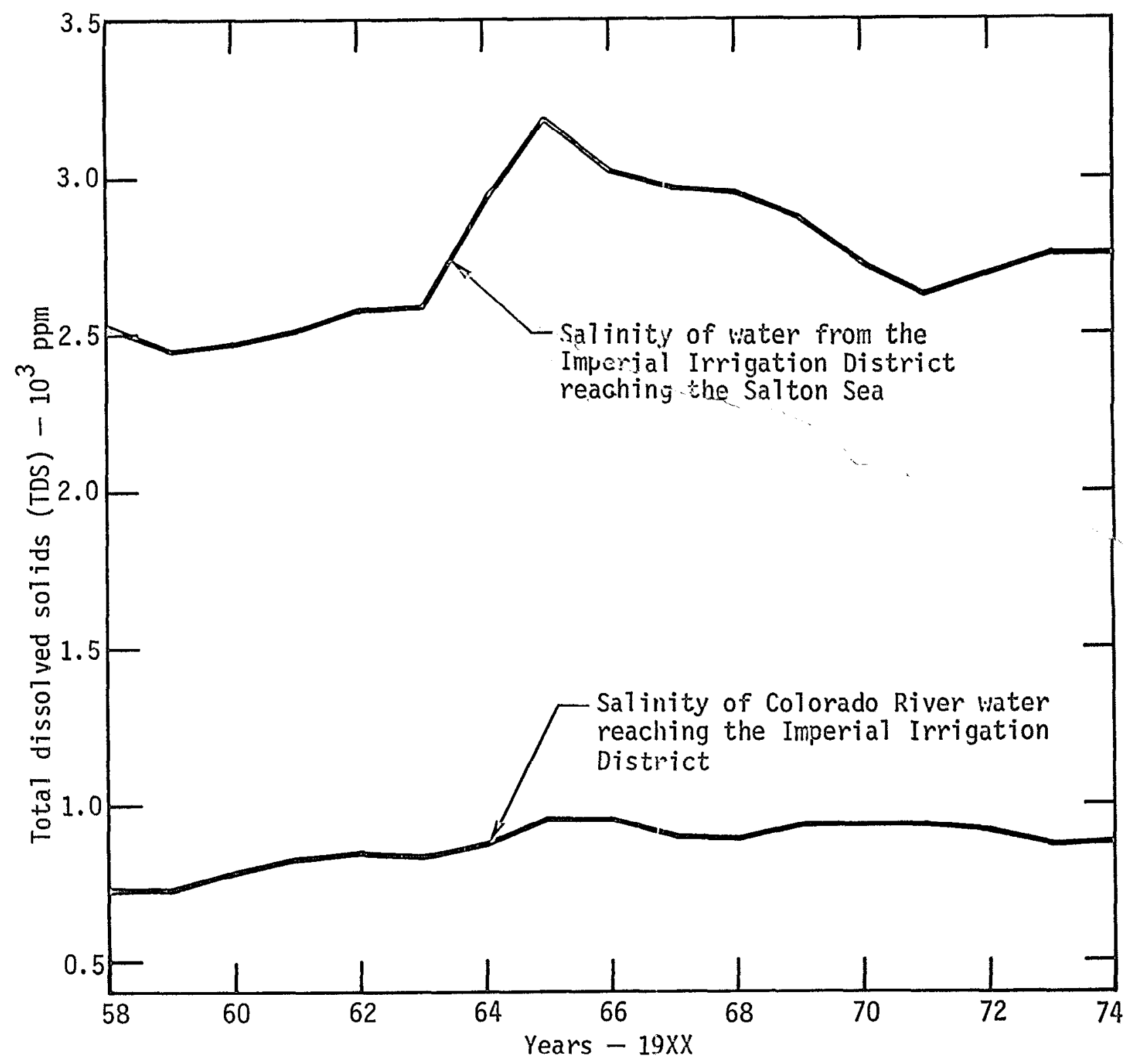

Fig. 3.3. Salinities of water entering and leaving the Imperial Irrigation District (excludes water and salt from lexico). ${ }^{5}$ 
control measures, may exceed 1,000 ppm TDS by 1990. And as salinity increases, crop yields should decrease unless corrective steps are taken. ${ }^{10}$ Another problem area has been the release of untreated sewage into the New River on the Mexican side of the border. This problem should be resolved when a new sewage treatment plant is completed by the Mexican government.

Effluent flows from agricultural lands affect water quality adversely by increasing the mineral burden of the New and Alamo Rivers and by contributing other pollutants (e.g., pesticides and nutrients) to the runoff that eventually ends up in the rivers. Because of the salt problem in the valley, water analyses have focused on the dissolved ionic constituents of the inflowing and outflowing waters to obtain a saltbalance for the valley. In recent years those analyses have shown that more salt has been removed from the valley than has been brought in, resulting in a positive balance. In 1974 the salt balance of waters in the irrigation district ${ }^{5}$ showed that 534,326 more tons of salt were discharged from the district than were brought in. The excess salt is derived principally from leaching of soils plus contributions from saline ground water. 4
Alterations in the dissolved constituents present in water moving through the irrigation system axe given in Fig. 3.4. According to Kaddah and Rhoades, ${ }^{4}$ the smaller relative amounts of $\mathrm{HCO}_{3}^{-}, \mathrm{SO}_{4}^{=}$, and $\mathrm{Ca}^{++}$in the drainage waters are probably caused by the precipitation of $\mathrm{CaCO}_{3}$ and CaSo. A bar graph of the ionic composition of Salton Sea water indicates a relatively minor amount of $\mathrm{HCO}_{3}^{-}$; the dominant ions are $\mathrm{Na}^{+}$ and $\mathrm{C} 1$ -

\subsection{SALTON SEA}

The initial filling of the Salton Sea occurred in the years 1904 to 1907 when control works on the Colorado River, meant to regulate diversions of river water to the Imperial Valley, failed and allowed most of the river to flow into what was called the Salton Sink. ${ }^{3}$ Since then the sea has been sustained by agricultural drainage waters and its primary beneficial use is the storage of agricultural waste waters. Its status as a depository for waste waters was guaranteed when, in 1924, President Coolidge withdrew all public lands in the Salton Sea area that were below an elevation of $244 \mathrm{ft}$, establishing a public water reserve. 12 In 1928 the President extended the reserve by withdrawing a11 public lands below an elevation of $220 \mathrm{ft}$. 

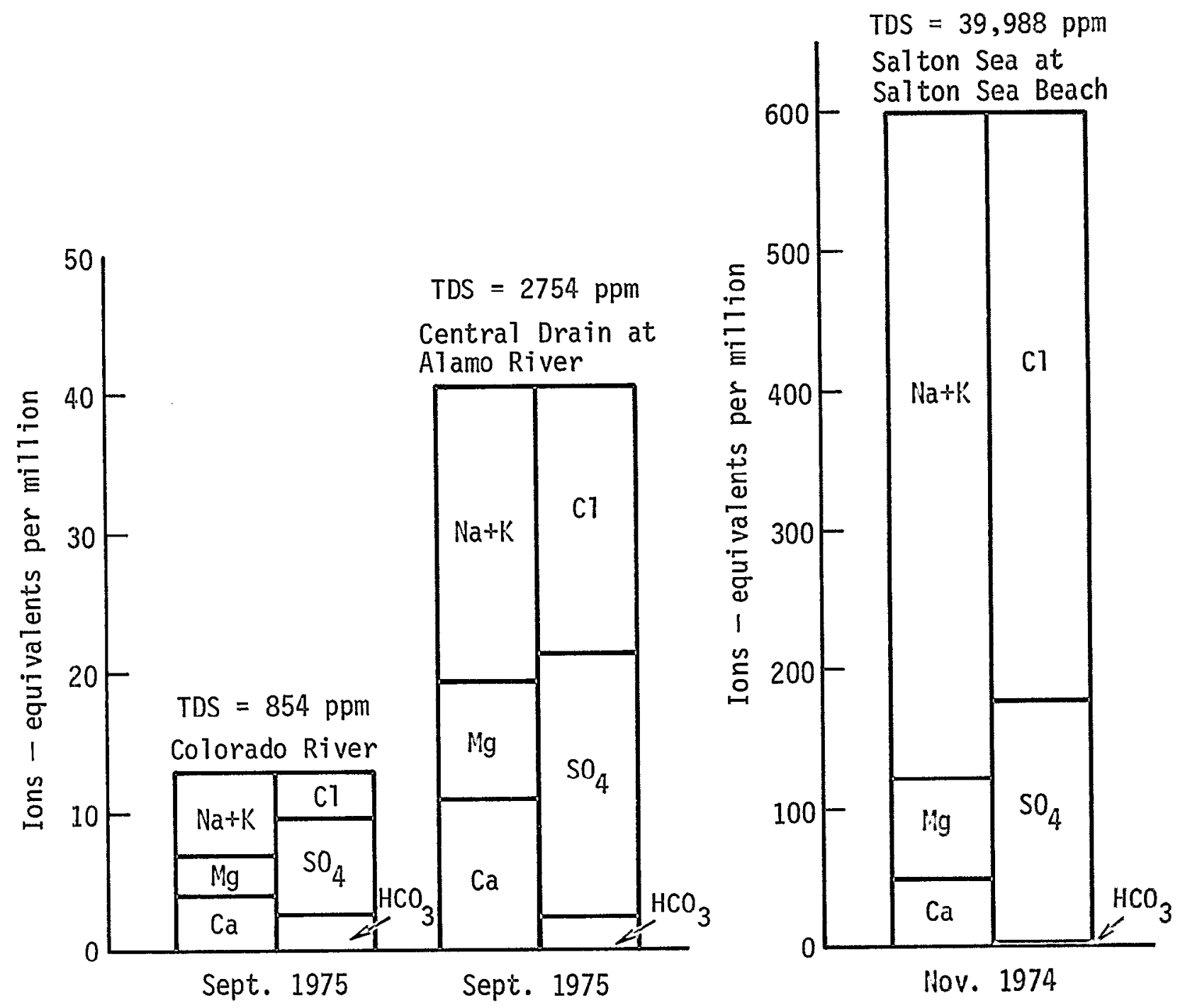

Fig. 3.4. Constituents of Colorado Fiver water, Central Drain water, and Salton Sea water. 5,11

Inflows to the sea have been partially balanced by evaporation that has averaged over 1,200,000 af per year. ${ }^{3}$ Whenever the evaporation does not keep pace with inflows from the Coachella and Imperial Valleys, the level of the sea rises. The historic relationship between surface elevation and area are depicted in Fig. 3.5. Continued rising of the sea has caused flooding and drainage problems along its southern edge.
This situation is likely to continuc until irrigation and cropping practices are a.t.tered.

Directly related to the amount of inrlows and evaporation is the change in the sea's salinity, which has risen to over 39,000 ppm TDS (see rig. 3.6). The sea's salinity can bie expected to increase gradually because of salt loading from braclish irrigation waste waters and volumatric reductions due to evaporation. 


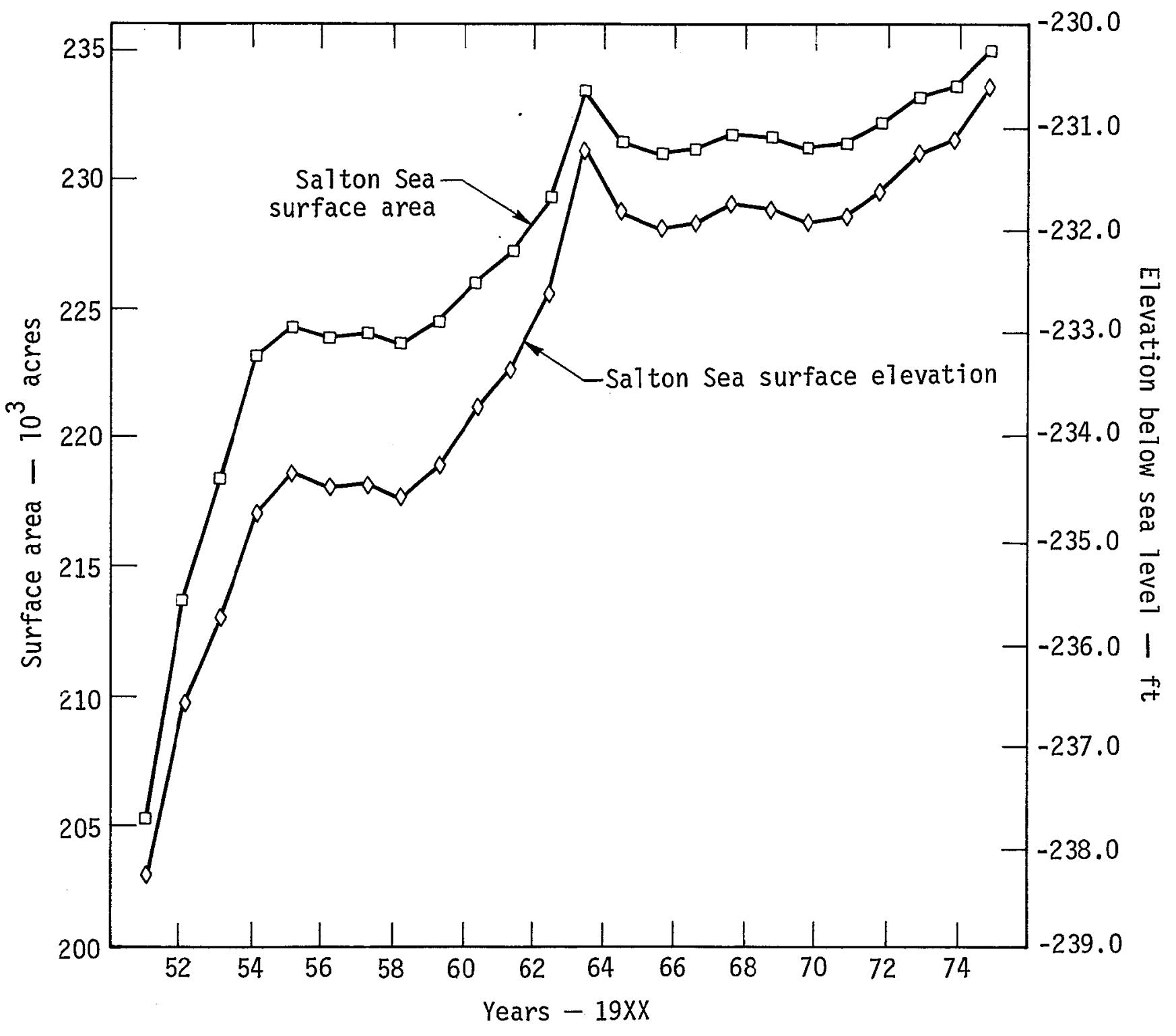

Fig. 3.5. Surface area and elevation of the Salton Sea during 1951-1974. 


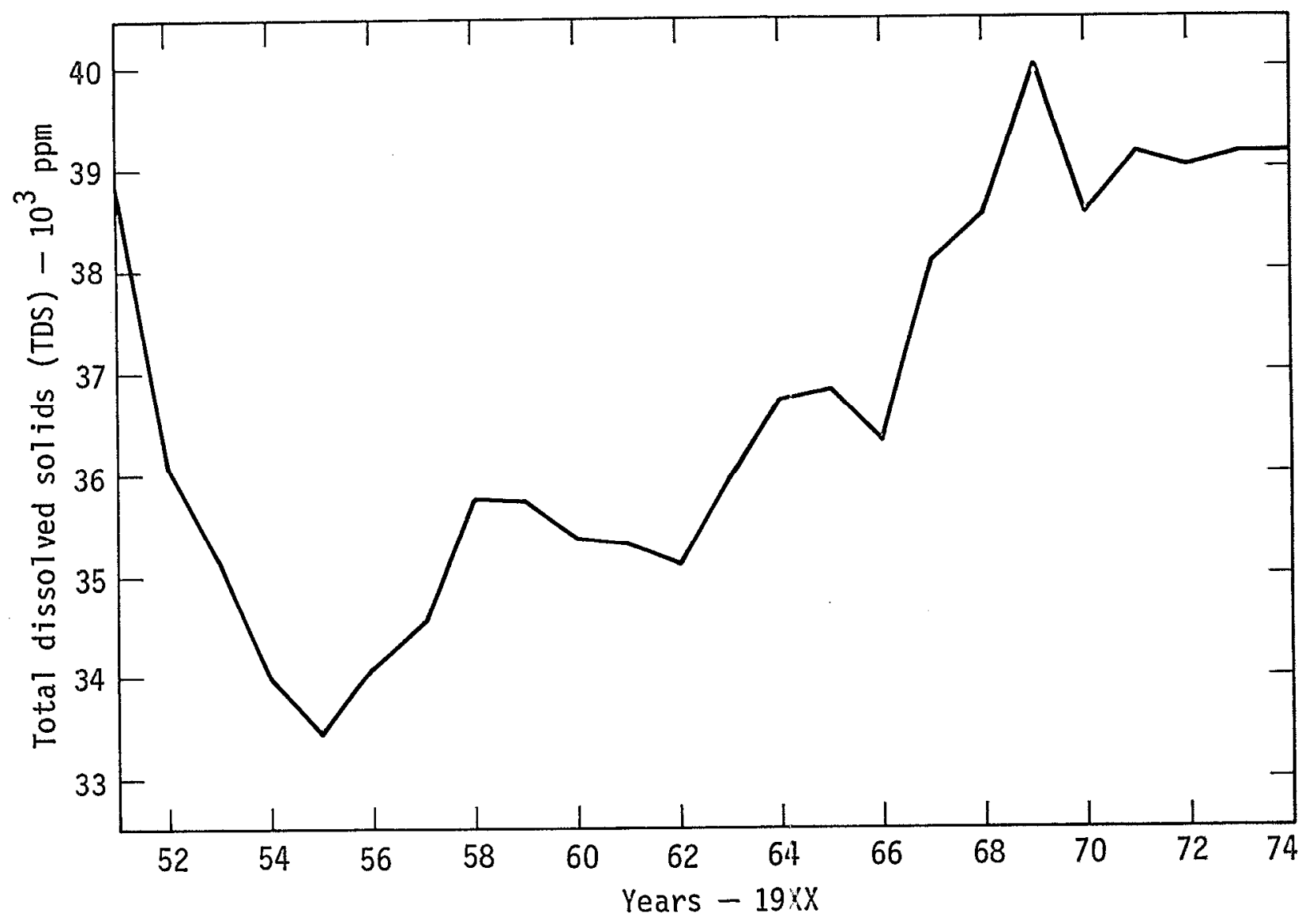

Fig. 3.6. Historic salinity of the Salton Sea. Salinity values are the average of samples taken from Bertram Station, Desert Beach, Sandy Beach, and Salton Sea Beach. 5 


\section{REFERENCES}

1. 0. J. Loeltz, B. Ireland, J. H. Robison, and F. If. Olmsted, Geohydrologic Reconnaissance of the Imperial Valley, California, Geological Survey Professional Paper 486-K, U.S. Government Printing Office, Washington, D.C. (1975).

2. L. C. Dutcher, W. F. Hardt, and W. R. Moyle, Jr., Preliminary Appraisal of Ground Water in Storage with Reference to Geothermal Resources in the Imperial Valley, California, Geological Survey Circular 649, Washington, D.C. (1972).

3. A. G. Hely, G. H. Hughes, and B. Ireland, Hydrologic Regimen of Salton Sea, California, Geologic Survey Professional Paper 486-C, U.S. Government Printing office, Washington, D.C. (1966).

4. M. T. Kaddah and J. D. Rhoades, "Salt and Water Balance in Imperial Valley, California," J. Am. Soil Sci. Soc., 40, 93 (1976).

5. Imperial Irrigation District, Imperial Irrigation District, Water Report, 1974, unpublished.

6. R. E. Glover, C. A. Nelson, and J. I. Sanders, "Colorado River Flow Management," J. Hydraul. Div., ASCE, 100 (HYII) 1519 (November, 1974).

7. Imperial Irrigation District, Rules and Regulations Governing the Distribution and Use of Water and Construction, Operation, and Maintenance of Canal and Drainage System of the Imperial Irrigation District, unpublished (1967).

3. V. E. Valentine, "Impacts of Colorado River Salinity," J. Irroig. Drain. Div., ASCE, 100 (IRH), 495 (1974).

9. U.S. Department of the Interior, Quality of Water, Colorado River Basin, Progress Rept. No. 7 (1975).

10. Po-Chuan Sun, An Economic Analysis on the Effects of Quantity and Quality of Irrigation Water on Agricultural Production in Imperial Valley, California, Ph.D. thesis, University of California, Davis (1971).

11. California Department of Water Resources, Water Data Information System, unpublished data (1976).

12. U.S. Department of the Interior and The Resources Agency of California, Salton Sea Project, California, Federal-State Reconnaissance Report (1969). 


\section{Section 4 \\ Biological Resources}

J. R. Kercher and Mary Buchanan

In the absence of man, the vegetation of the Imperial Valley would be creosote bush-bur sage desert communities. However, because of man's activities, there is a large irrigated agricultural district and a large inland salt water sea. Four ecological systems in the Imperial Valley will be considered in this chapter: agricultural, the Salton Sea quasi-marine ecosystem, wild1ife refuges and endangered species, and native desert communities.

\subsection{AGRICULTURE}

The unique climate of the Imperial Valley makes it one of the most valuable agricultural resources in the nation. In 1974, there vere 489,000 acres in agricultural production that generated a gross sales of $\$ 557$ million. Of this $\$ 557$ million, livestock and dairy accounted for $\$ 1.55$ million, field crops $\$ 284$ million, and vegetable crops $\$ 103$ million. ${ }^{1}$ These 489,000 acres are divided into more than 8000 parcels. ${ }^{2}$ It is the largest single area of irrigated agriculture in the Western Hemisphere. ${ }^{3}$ The climate of the Imperial Valley is hot and dry with an average annual rainfall of 2.7 inches $^{4}$ and a maximum temperature over $100^{\circ} \mathrm{F}$ for more than 110 days of the year. ${ }^{3}$ Thure is an average of 314 days between frosts and 12 days of frosts. ${ }^{4}$ The annual average relative humidity is below $30 \%$ with the summer month: of July, August, and September the hilhest. This is because the prevailin: vinds in the summer come from the south (Gulf of California); durins: the rest of the year the prowalliny: winds are from the vest. ${ }^{4}$

Probably the single most important problem for agriculture in the Imperial Valley is soil silinity. The irrigation system is intimatcly tied to this problem. The vater management aspects of the Imperial Valley are discussed in detail clickwhere. In this section the soll salinity problem is addressed explicitly.

The agricultural area under irrigation is shown in Fig. 4.1. Crops:

The crops grown in the valley during the years 1973 through 1975 are listed in Table 4-1. ${ }^{6}$ Table 4-2 describes the total area served by irrization. In terms of acreage the most important crops are alfalfa, 


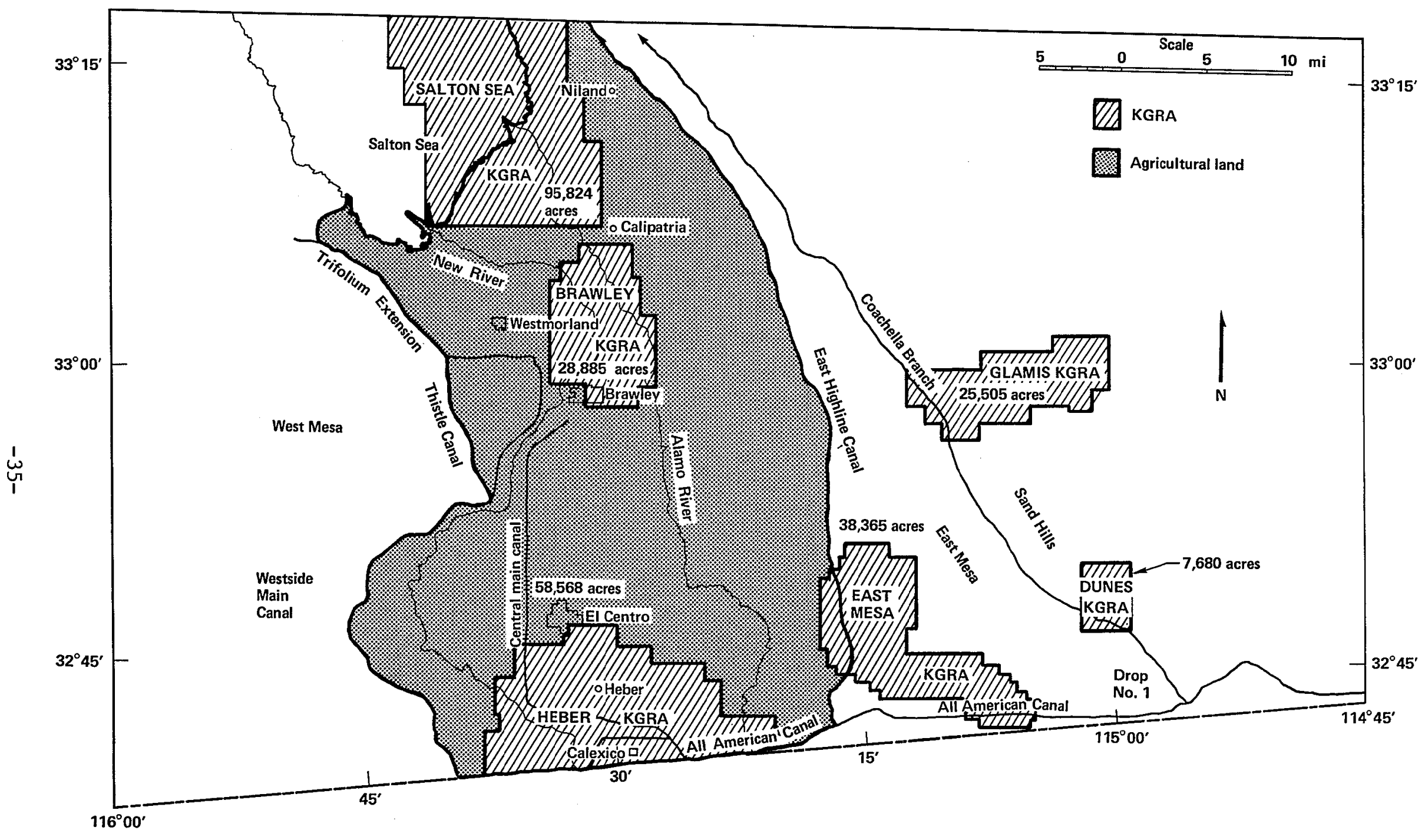

Fig. 4.1. Map of Imperial Valley. The six KGRAs are outlined on this map. Irrigated, agricultural lands are shown as the shaded areas. 
Table 4.1. Crop acreage ${ }^{a}$ in the Imperial Irrigation District. 6

\begin{tabular}{|c|c|c|c|}
\hline \multirow[b]{2}{*}{ Garden crops } & \multicolumn{3}{|c|}{ Acres } \\
\hline & 1975 & 1974 & 1973 \\
\hline Broccoli & 773 & 710 & 239 \\
\hline Broccoli (seed) & 17 & 11 & 17 \\
\hline Cabbage & 319 & 1429 & 626 \\
\hline Carrots & 5988 & 6385 & 5040 \\
\hline Carrots (seed) & 22 & 49 & 27 \\
\hline Cauliflower & 5 & -- & 64 \\
\hline Cauliflower (seed) & 45 & 73 & 42 \\
\hline Collards (seed) & 33 & 41 & -- \\
\hline Cucumbers & 981 & 503 & 233 \\
\hline Ear Corn & 4 & -- & 467 \\
\hline Endive & 20 & 7 & - \\
\hline Endive (seed) & 22 & -- & - \\
\hline Garlic & 1395 & 708 & 678 \\
\hline Herbs, mixed & 40 & 28 & 6 \\
\hline Lettuce & 44912 & $48 \quad 376$ & 40701 \\
\hline Lettuce (seed) & 118 & -- & - \\
\hline Lettuce, Romaine & 113 & 34 & 167 \\
\hline \multicolumn{4}{|l|}{ Melons } \\
\hline Cantaloupes & 7559 & 8888 & 9570 \\
\hline Cantaloupes (seed) & 45 & 10 & - \\
\hline Crenshaw & 363 & 143 & 293 \\
\hline Honeyder & 842 & 148 & 369 \\
\hline Mixed & 60 & 4 & 75 \\
\hline Watermelons & 2472 & 1573 & 2659 \\
\hline Watermelons (seed) & -- & 28 & -- \\
\hline Mustard & 310 & 225 & 339 \\
\hline Okra & 23 & 46 & 20 \\
\hline Okra (seed) & 45 & 6 & -- \\
\hline Onions & 7509 & 6273 & 4462 \\
\hline Onions (seed) & 1248 & 1469 & 858 \\
\hline Parsley (seed) & 20 & - & - \\
\hline Parsnips & 30 & 45 & - \\
\hline Peas & 223 & 40 & -- \\
\hline Peas (seed) & 136 & -- & -- \\
\hline Rapini & 259 & 280 & 136 \\
\hline Rutabagas & 45 & 20 & - \\
\hline Squash & 1287 & 970 & 1241 \\
\hline Squash (seed) & - & 17 & - \\
\hline Tomatoes & 5736 & 2909 & 2257 \\
\hline Tomatoes (seed) & 132 & - & - \\
\hline Turnips & 62 & 53 & - \\
\hline Vegetables, mixed & 212 & 122 & 199 \\
\hline Vegetables, mixed (seed) & 35 & 18 & 29 \\
\hline Waterlilies & 16 & 25 & 20 \\
\hline Totals & 83476 & 81666 & 70834 \\
\hline
\end{tabular}


Table 4.1. (Continued)

\begin{tabular}{|c|c|c|c|}
\hline \multirow[b]{2}{*}{ Field crops } & \multicolumn{3}{|c|}{ Acres } \\
\hline & 1975 & 1974 & 1973 \\
\hline Alfalfa & 158784 & 155608 & $174 \quad 567$ \\
\hline Alfalfa (seed) & 627 & 2383 & 1660 \\
\hline Alicia grass & 2900 & 2797 & 2722 \\
\hline Barley & 3481 & 5358 & 17433 \\
\hline Bermuda grass & 2158 & 2403 & 1968 \\
\hline Bermuda grass (seed) & 1046 & 964 & 964 \\
\hline Cotton & 43000 & 78808 & 36857 \\
\hline Flax & 145 & 40 & 80 \\
\hline Oats & 275 & 1002 & 1245 \\
\hline Rape & -- & 46 & - \\
\hline Rye grass & 8766 & 8875 & 17456 \\
\hline Rye grass (seed) & 203 & 294 & 509 \\
\hline Safflower & 170 & $\rightarrow$ & 16 \\
\hline Sesbania & 221 & -- & - \\
\hline Sesbania (seed) & - & -- & 79 \\
\hline Sorghum grain & 24271 & 31610 & 39389 \\
\hline Sorghum silage & 560 & 417 & 1032 \\
\hline Soy beans & - & - & 2 \\
\hline Sudan grass & 13047 & 14450 & 13224 \\
\hline Sugar beets & 71425 & 69108 & 69812 \\
\hline Wheat & 155575 & 101499 & $94 \quad 407$ \\
\hline Totals & $486 \quad 654$ & 475662 & 473422 \\
\hline \multicolumn{4}{|l|}{ Permanent crops } \\
\hline Apricots & 22 & 22 & 22 \\
\hline Asparagus & 4426 & 5066 & 5034 \\
\hline Citrus & & & \\
\hline Grapefruit & 600 & 657 & 618 \\
\hline Lemons & 968 & 967 & 836 \\
\hline Mixed & 292 & 285 & 380 \\
\hline Oranges & 409 & 444 & 444 \\
\hline Tangerines & 256 & 268 & 282 \\
\hline Dates & 76 & 76 & 83 \\
\hline Duck ponds (feed) & 6809 & 7020 & 7348 \\
\hline Fish farms & 425 & 465 & 426 \\
\hline Fruit, mixed & 100 & 73 & 73 \\
\hline Ornamental shrubs & 8 & 8 & 8 \\
\hline Pasture, permanent & 997 & 556 & 749 \\
\hline Peaches & 35 & 35 & 35 \\
\hline Pecans & 47 & 47 & 47 \\
\hline Totals & 15470 & 15989 & 16385 \\
\hline Total acres of crops & 585600 & 573317 & 560641 \\
\hline
\end{tabular}

${ }^{\mathrm{a}}$ Crops are 1isted for the year in which they are predominantly harvested. 
Table 4.2. Summary of area served by Imperial Irrigation District. ${ }^{6}$

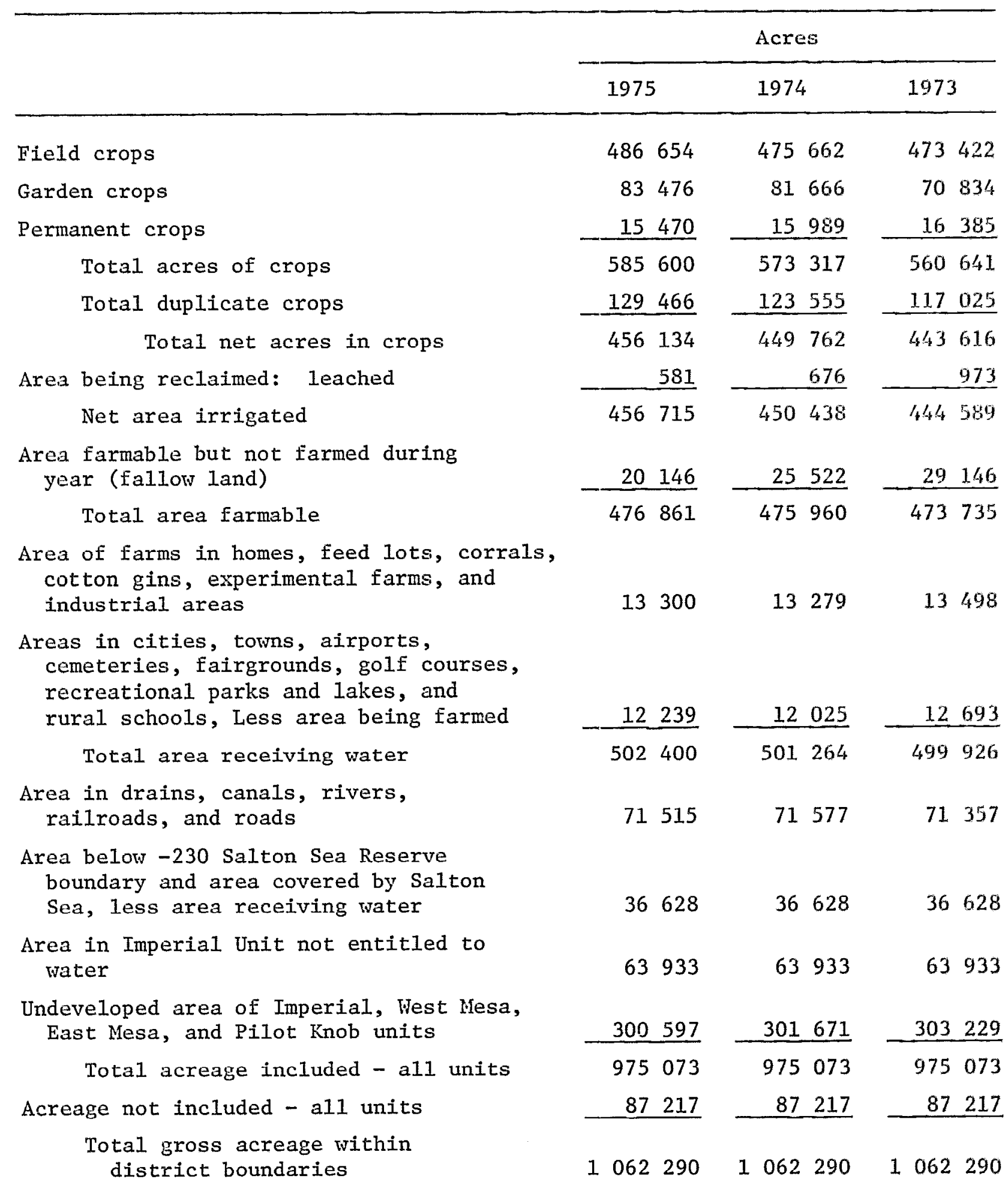




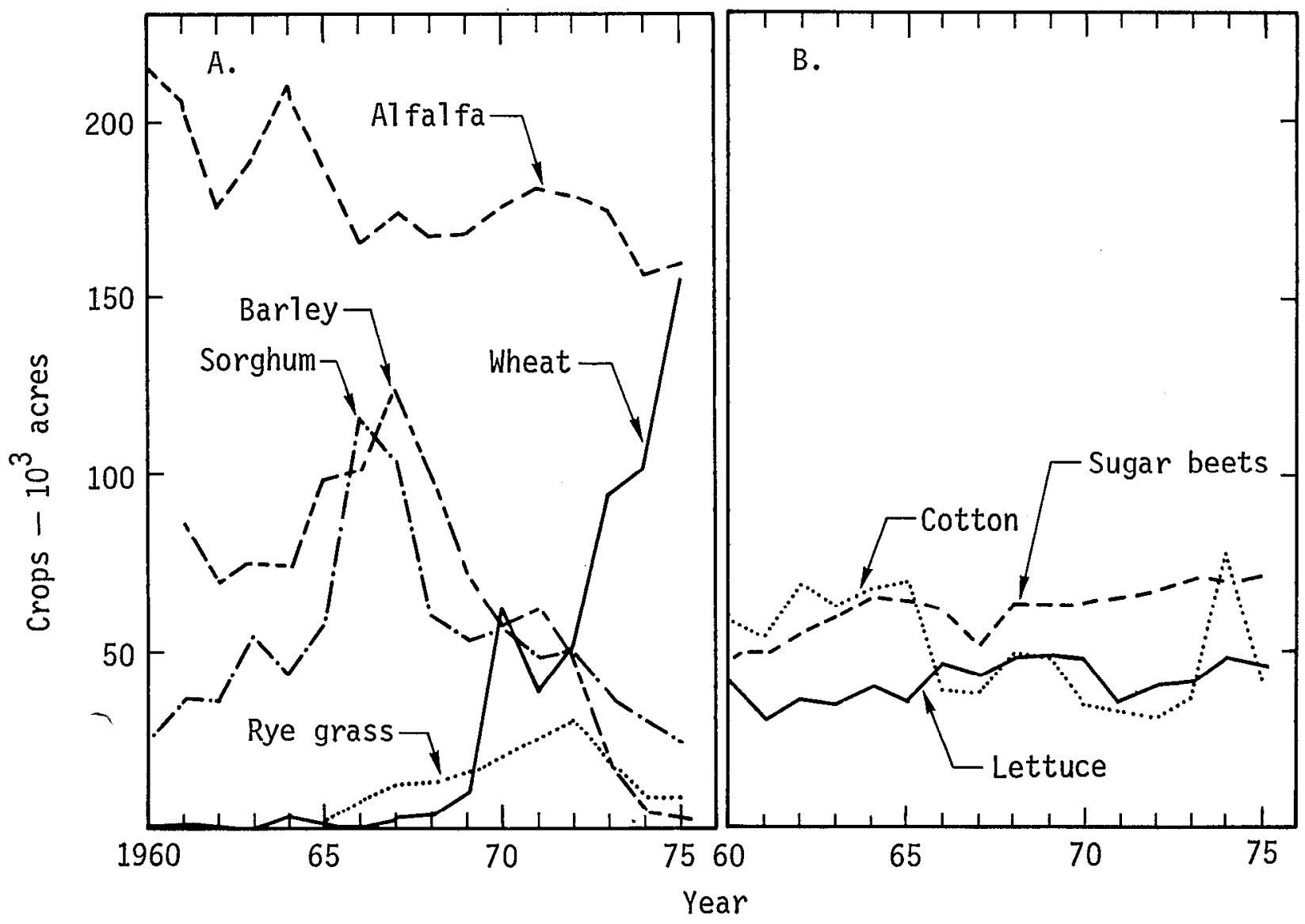

Fig. 4.2. Annual crop production in acres in the Imperial Irrigation District.

A) The changes in the last 15 years in grain production in the Imperial

Valley. B) Fluctuations in a vegetable crop (lettuce) and two field crops

(sugar beets and cotton) over the last 15 years. Data courtesy of the Imperial Irrigation District. 7

wheat, sugar beets, lettuce, cotton, and sorghum grain. These 6 crops are grown on 498,000 acres (includes double cropping) out of a total of 585,600 acres for 74 crops in 1975 . In Fig. 4.2 we show the yearly variation in acreage for the most prominent crops in the last 15 years. ${ }^{7}$ These variations reflect the farmers' responses to market fluctuations in the price of crops and the price of Eactors of production. Notice the inistorical growth of wheat and the reduction in sorghum and barley in recent years. In Fig. 4.3, the planting and harvesting schedules for Imperial Valley are displayed. 8,9 From this figure, it appears that most vegetable crops are planted in the fall months, mature during the fall and winter, and are then harvested in the winter and spring. Lettuce, a major crop, is harvested in the early winter (December and January). Wheat and sugar beets are harvested in spring. Good success has been 


\begin{tabular}{|c|c|c|c|c|c|c|c|c|c|c|c|c|}
\hline Crop & Aug & Sep & $0 c t$ & Nov & Dec & Jan & $\mathrm{Feb}$ & Mar & Apr & May & Jun & JuT \\
\hline \multicolumn{13}{|l|}{ Major crops } \\
\hline \multicolumn{13}{|c|}{ Nseed, icroinst) } \\
\hline \multicolumn{13}{|l|}{ Broccoli } \\
\hline \multicolumn{13}{|l|}{ Cantaloupes } \\
\hline \multicolumn{13}{|l|}{ Carrots } \\
\hline \multicolumn{13}{|l|}{ Garlic } \\
\hline \multirow{2}{*}{\multicolumn{13}{|c|}{ Lettuce }} \\
\hline \multirow{2}{*}{\multicolumn{13}{|c|}{ Onions }} \\
\hline \multirow{2}{*}{\multicolumn{13}{|c|}{ Dehydrator }} \\
\hline & & & & & & & & & & & & \\
\hline \multicolumn{13}{|l|}{ Fresh market } \\
\hline \multicolumn{13}{|l|}{ Seed } \\
\hline \multicolumn{13}{|l|}{ Tomatoes } \\
\hline \multicolumn{13}{|l|}{ Open, cannery } \\
\hline \multicolumn{13}{|l|}{ Brushed, staked } \\
\hline \multicolumn{13}{|l|}{ Watermelons } \\
\hline \multicolumn{13}{|l|}{ Minor crops } \\
\hline \multicolumn{13}{|l|}{ Cabbage } \\
\hline \multicolumn{13}{|l|}{ Cucumbers } \\
\hline \multicolumn{13}{|l|}{ Melons } \\
\hline \multicolumn{13}{|l|}{ Casaba } \\
\hline \multicolumn{13}{|l|}{ Honeydew } \\
\hline \multicolumn{13}{|l|}{ Persian } \\
\hline \multicolumn{13}{|l|}{ Rapini } \\
\hline \multicolumn{13}{|l|}{ Okra } \\
\hline \multicolumn{13}{|l|}{ Onions - bunching } \\
\hline \multicolumn{13}{|l|}{ Romaine } \\
\hline \multicolumn{13}{|l|}{ Sweet corn } \\
\hline Squash & & & & & & & & & & & & \\
\hline Summer & $\Delta$ & III & & & IV & $E$ & & & & & & \\
\hline Banana & & & & & & 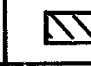 & & & & & & $\pi$ \\
\hline
\end{tabular}

Fig. 4.3. Production calendars for Imperial County crops.8,9 These calendars show time of year for land preparation and plinting, growth, and harvesting of the major crops of Imperial County. A) Verzetable crops. B) Field crops. 
Field crops calendar

Alfalfa for hay

(3 to $4 \mathrm{yr}$. crop)

Alfalfa seed

Cereals

Cotton

Flax

Sesbania

Sorghums (forage)

Sorghum (grains)

Sugar beets

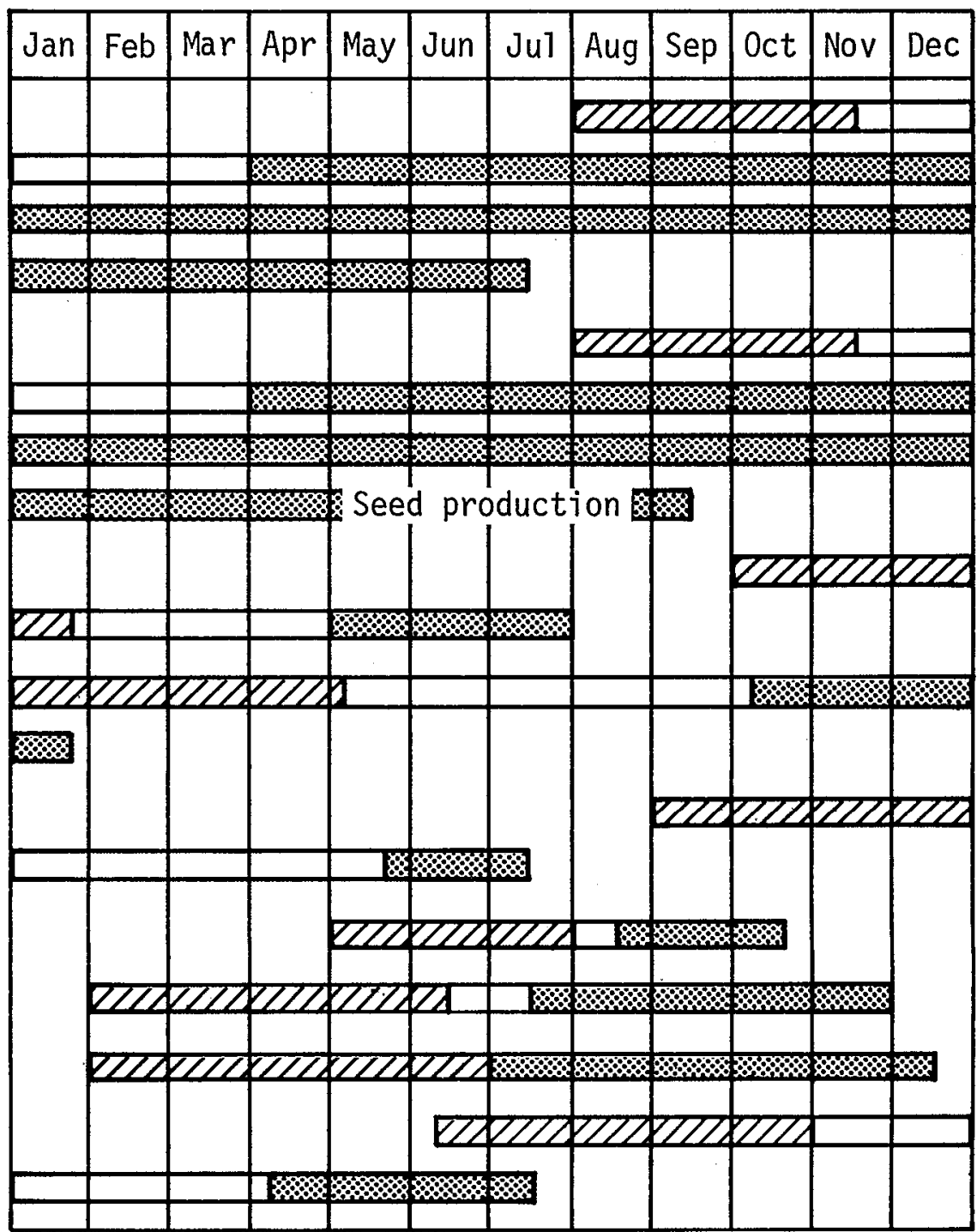

Land preparation and planting

प्याप्य

Cultivation, growth, and lay-by

Harvest

$\ldots$

reported in using this calendar : sequence to identify crops from high altitude and earth resources technology satellite (ERTS) images. ${ }^{2,3}$ In fact, a newly introduced crop in the Imperial Valley was detected by
Johnson and Coleman ${ }^{2}$ because it did not fit into this calendar.

Table $4.3^{10}$ lists the major crops in order of acreage for each of the four KGRAs that are either entirely or partly on agricultural 1.and. The 
Table 4.3. Major crops grown in KGRAs by approximate relative acreage. $a, b$

\begin{tabular}{|c|c|c|c|c|c|c|c|c|c|c|c|c|c|}
\hline KGRA & Wheat & Alfalfa & $\begin{array}{l}\text { Sugar } \\
\text { beets }\end{array}$ & Lettuce & Cotton & Sorghum & Melons & Tomatoes & Onions & $\begin{array}{l}\text { Rye } \\
\text { grass }\end{array}$ & $\begin{array}{l}\text { Sudan } \\
\text { grass }\end{array}$ & Carrots & Asparagus \\
\hline Niland & 1 & 3 & 2 & 6 & 4 & 5 & 9 & 8 & 7 & 10 & 11 & & \\
\hline Brawley & 1 & 2 & 3 & 4 & 5 & 6 & 7 & 8 & 11 & 9 & 10 & & \\
\hline Heber & 1 & 2 & 3 & 4 & 6 & 5 & 7 & 9 & 8 & 10 & 11 & 13 & 12 \\
\hline East Mesa & 2 & 1 & 3 & 4 & 5 & 6 & 7 & & & 9 & & 8 & \\
\hline
\end{tabular}

$\vec{B}^{\mathrm{B}}$ Reference 10 .

bumbers refer to relative order of each crop's acreage in that hGRA; 1 represents the greatest acreagc. 
pattern is quite consistent for each KGRA compared to the valley average taken as a whole.

In Table $4.4,^{10}$ are listed the water requirements, harvest time, and biocide applications for each major crop. Notice that cotton is defoliated once each year.

Soils and Salinity

Most of the soils of the Imperial Valley are alluvial deposits of the
Colorado River in the Salton Trough. These were deposited at the time that the Imperial Valley was part of the Colorado River delta. The deposits are up to 20,000 feet deep. 5,11 No soil profile exists. Instead, the horizons are those of deposition, not true horizons of soil development. 12 This accumulation of alluvial material, mainly clays with sand lenses, means that drainage is poor with no grave1 or sand strata extending over any

Table 4.4. Major crops in the Imperial Valley with water requirements, harvest dates, and biocide applications.10

\begin{tabular}{|c|c|c|c|}
\hline Crop & $\begin{array}{l}\text { Water } \\
\text { requirements, ft }\end{array}$ & Harvest & Pesticides \\
\hline Wheat & 3 & May, June, July & $\begin{array}{l}1 \text { Herbicide application } \\
1 \text { Insecticide application }\end{array}$ \\
\hline Alfalfa & 7 & Al1 Year & $\begin{array}{l}1 \text { Herbicide application } \\
4 \text { Insecticide applications }\end{array}$ \\
\hline Cotton & 5.5 & $\begin{array}{l}\text { Nov. through } \\
\text { Feb. }\end{array}$ & $\begin{aligned} & 2 \text { Herbicide applications } \\
& 10 \text { Insecticide applications } \\
& 1 \text { Defoliation }\end{aligned}$ \\
\hline Sorghums & 4 & $\begin{array}{l}\text { July through } \\
\text { Nov. }\end{array}$ & $\begin{array}{l}1 \text { Herbicide application } \\
1 / 2 \text { Insecticide application }\end{array}$ \\
\hline Sugar beets & 6 & $\begin{array}{l}\text { April through } \\
\text { July }\end{array}$ & $\begin{array}{l}\text { I Herbicide application } \\
5 \text { Insecticide applications }\end{array}$ \\
\hline Asparagus & 6 & $\begin{array}{l}\text { Jan. through } \\
\text { April }\end{array}$ & $\begin{array}{l}1 \text { Herbicide application } \\
2 \text { Insecticide applications }\end{array}$ \\
\hline Melons & 3 & May, June, Oct. & 3 Insecticide applications \\
\hline Carrots & 3.5 & $\begin{array}{l}\text { Nov. through } \\
\text { June }\end{array}$ & $\begin{array}{l}2 \text { Herbicide applications } \\
2 \text { Insecticide applications }\end{array}$ \\
\hline Lettuce & 4.5 & $\begin{array}{l}\text { Dec. through } \\
\text { Apri1 } 10\end{array}$ & $\begin{array}{l}1 \text { Herbicide application } \\
10 \text { Insecticide applications }\end{array}$ \\
\hline Onions & 4.5 & $\begin{array}{l}\text { May through } \\
\text { June }\end{array}$ & $\begin{array}{l}1 \text { Herbicide application } \\
2 \text { Insecticide applications }\end{array}$ \\
\hline Tomatoes & 3 & $\begin{array}{l}\text { May through } \\
\text { June }\end{array}$ & 12 Insecticide applications \\
\hline
\end{tabular}


Table 4.5. Physical and chemical properties of Imperial Valley soil series samples. ${ }^{4}$

\begin{tabular}{|c|c|c|c|c|c|c|}
\hline \multirow{2}{*}{ Percent of cultivated land } & \multirow{2}{*}{ Imperial } & \multicolumn{2}{|c|}{ Holtville } & \multirow{2}{*}{$\frac{\text { Meloland }}{12}$} & \multicolumn{2}{|c|}{ Indio } \\
\hline & & 2 & & & & \\
\hline Description $^{a}$ & sic & sic & sic-ss & vfsl & 1 & vfsl-ss \\
\hline $\mathrm{pH}$ & 7.7 & 7.7 & 7.7 & 7.6 & 7.7 & 8.1 \\
\hline Sand $^{\mathrm{b}}(\%)$ & 3.4 & 10.8 & 8.0 & 66.3 & 21.3 & 35.6 \\
\hline $\operatorname{silt}^{b}(\%)$ & 49.0 & 41.1 & 43.1 & 23.5 & 63.3 & 52.8 \\
\hline clay $^{\mathrm{b}}(\%)$ & 47.6 & 48.1 & 48.9 & 10.2 & 15.4 & 13.1 \\
\hline Field capacity ${ }^{b}(\%)$ & 34.9 & 32.1 & 32.4 & 10.7 & 20.7 & 16.5 \\
\hline Wilting coefficient ${ }^{b}(\%)$ & 20.9 & 18.1 & 18.4 & 5.4 & 7.7 & 7.0 \\
\hline Infiltration rate (in/hr) & $0.051 \pm .01$ & $0.092 \pm .039$ & $0.396 \pm .318$ & $0.315 \pm .083$ & $0.208 \pm .116$ & $0.267 \pm .163$ \\
\hline Electrica1 conductivity ${ }^{\mathrm{c}}(\mathrm{mmho} / \mathrm{cm})$ & 4.9 & 6.8 & 5.0 & 7.0 & 5.0 & 19.0 \\
\hline Cation exchange capacity ${ }^{c}$ (meq/1) & 34.2 & 27.5 & 29.5 & 13.2 & 15.5 & 14.4 \\
\hline Exchangeable $\mathrm{Na}^{\mathrm{C}}(\%)$ & 14 & 12 & 21 & 15 & 30 & 26 \\
\hline Exchangeable $\mathrm{K}^{\mathrm{c}}(\%)$ & 4 & 5 & 4 & 3 & 6 & 4 \\
\hline Exchangeable $\mathrm{Ca}^{\mathrm{C}}(\%)$ & 23 & 30.5 & 24.3 & 32.4 & 21.4 & 26.9 \\
\hline Exchangeable $\mathrm{Mg}^{\mathrm{C}}(\%)$ & 11.9 & 19.6 & 12.0 & 19.7 & 15.1 & 28.0 \\
\hline
\end{tabular}

$a_{\text {sic }}=$ silty clay, $s s=$ sandy substrate, vfsl = very fine sandy loam, $1=10 a m$.

$\mathrm{b}_{\mathrm{A}}$ and $\mathrm{Cl}$ horizon.

$\mathrm{c}_{\text {Top }} 12$ to 17 in. 
appreciable area. This in turn has meant that salt has accumulated in the system and can rise to the rooting zone. 5 In well-managed soils in the Imperial Valley, typical values of soil salinity are 3 to 4 mmos electroconductivity (soil extract) in the top foot with up to 7 to 8 mmhos at a depth of 2 to 3 feet. 13

$$
\text { Perrier et al. } 4 \text { have classified }
$$
the soils in the irrigated area of the Imperial Valley as Torriorthents and Torrifluvents. The land is nearly flat with a slope of 5 feet in a mile. $^{14}$ Table 4.5 lists the 6 phases in 4 soil series that make up $30 \%$ of the cultivated area. This table is abstracted from Perrier et al. 4 As expected, the sandy soils have the lowest water capacity (field capacity minus wilting coefficient) and the highest infiltration rates. The exception is that Holtville silty clay over a sandy substratum has a high infiltration rate. The Imperial and Holtville soils had cation exchange capacities typical of arid region soils; Meloland and Indio soils have cation exchange capacities typical of humid regions soils. ${ }^{15}$ Soils with more than $15 \%$ of their total exchange capacity occupied by $\mathrm{Na}$ are classified as saline-alkali. Those with less than $15 \%$ are classed as saline. In both classifications the $\mathrm{pH}$ of the soil is less than 8.5. More detailed soil analyses can be found in Ref. 4.

The Imperial Irrigation District ${ }^{16}$ has described the soil characteristics and qualities for al1 of Imperial County. Summarized in Table 4.6 are some aspects of their descriptions of agricultural land. Note that they classify the soil types into associations and that there is not a one to one correspondence between this classification and the nomenclature of Perrier et al. In all these soils, subsurface drainage must be used to maintain the water table to 4 to 5 feet under irrigation. For all the soils 1 isted, the choice of plants is limited by wetness, since the soils are poorly drained.

Salinity Control Practices and Associated Problems

The major problem of salinity control is attacked by

- The use of relatively good Colorado River water (under 900 ppin) to leach salts from the soil and removing them by a 1400-mi-long drainage system that drains into the New and Alamo Rivers, which in turn empty into the Salton Sea.

- Installation of a tile or plastic drainage system at a depth of 6 ft to carry off excess leaching water. 
Table 4.6. Properties of Imperial Soils. ${ }^{16}$

\begin{tabular}{|c|c|c|c|c|c|c|}
\hline Soil & $\begin{array}{l}\% \text { Irrigated } \\
\text { land }\end{array}$ & Description $^{a}$ & $\begin{array}{c}\text { Subsoil } \\
\text { permeability }\end{array}$ & $\begin{array}{l}\text { Inherent } \\
\text { fertility }\end{array}$ & $\begin{array}{l}\text { Land capability, } \\
\text { under irrigation }\end{array}$ & $\begin{array}{l}\text { Infiltration } \\
\text { rate when wet }\end{array}$ \\
\hline $\begin{array}{l}\text { Ho1tville } \\
\text { association, wet }\end{array}$ & 15 & & & & & \\
\hline Holtville & & sic & $\begin{array}{l}\text { Slow over } \\
\text { mod. rapid }\end{array}$ & $\mathrm{High}$ & III $\vee 5$ & Very slow \\
\hline $\begin{array}{l}\text { Glendale-Imperial } \\
\text { association, wet }\end{array}$ & 29 & & & & & \\
\hline $\begin{array}{l}\text { Glendale } \\
\text { Imperial }\end{array}$ & & $\begin{array}{l}\mathrm{ci} \\
\mathrm{sic}\end{array}$ & $\begin{array}{l}\text { Mod. slow } \\
\text { Slow }\end{array}$ & $\begin{array}{l}\text { High } \\
\text { High }\end{array}$ & $\begin{array}{lll}\text { II } & \text { w } & 5 \\
\text { IV } & \text { w } & 5\end{array}$ & Very slow \\
\hline $\begin{array}{l}\text { Imperial } \\
\text { association, wet }\end{array}$ & 23 & & & & & \\
\hline Imperial & & sic & Slow & High & IV $w 5$ & Very slow \\
\hline $\begin{array}{l}\text { Gila-Vinton } \\
\text { association, wet }\end{array}$ & 9 & & & & & \\
\hline $\begin{array}{l}\text { Gila } \\
\text { Vinton }\end{array}$ & & $\frac{1}{f} \leq 1$ & $\begin{array}{l}\text { Mod. slow } \\
\text { Moderate }\end{array}$ & $\begin{array}{l}\text { Hoderate } \\
\text { Low }\end{array}$ & $\begin{array}{l}\text { II } w 5 \\
\text { II I } w 4\end{array}$ & $\begin{array}{l}\text { Slow } \\
\text { slow }\end{array}$ \\
\hline $\begin{array}{l}\text { Meloland-Gila } \\
\text { association, wet }\end{array}$ & 13.5 & & & & & \\
\hline $\begin{array}{l}\text { Meloland } \\
\text { Gila }\end{array}$ & & $\begin{array}{l}1 \\
1\end{array}$ & $\begin{array}{l}\text { Slow } \\
\text { Slow }\end{array}$ & $\begin{array}{l}\text { Moderate } \\
\text { Moderate }\end{array}$ & 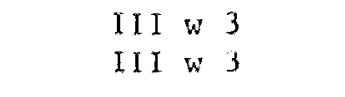 & $\begin{array}{l}\text { Very slow } \\
\text { Slow }\end{array}$ \\
\hline $\begin{array}{l}\text { Niland-Imperial } \\
\text { association, wet }\end{array}$ & 4 & & & & & \\
\hline $\begin{array}{l}\text { Niland } \\
\text { Imperial }\end{array}$ & & $\begin{array}{l}\text { gls } \\
\text { sic }\end{array}$ & $\begin{array}{l}\text { Slow } \\
\text { Slow }\end{array}$ & $\begin{array}{l}\text { Lori } \\
\text { High }\end{array}$ & $\begin{array}{l}\text { IV } w 5 \\
18=5\end{array}$ & $\begin{array}{l}\text { very } \& 10 \mathrm{x} \\
\text { gery } \$ 10 \mathrm{~m}\end{array}$ \\
\hline
\end{tabular}

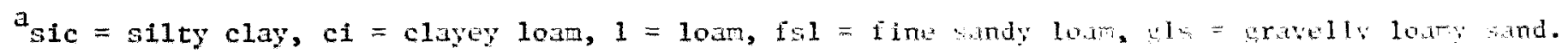

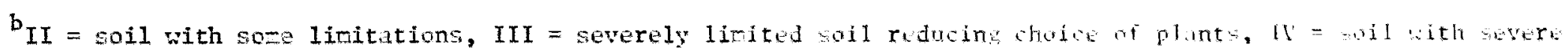

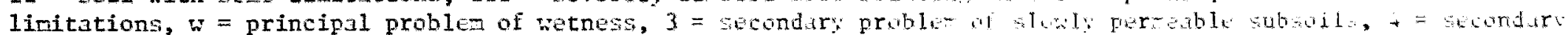
problem of coarse textures, $5=$ secondary problem of fine testures. 
- Establishment of salt tolerant crops.

- The planting of crops on doublerow, sloping beds. 5

The buried drainage system is subject to failure by clogging. This can occur from silt deposits or root growth $^{17}$ or can occur by chemical accumulation of iron and manganese oxides. $^{18}$ Physical clogging is removed by mechanical Roto-Rooters. *17 Chemical clogging is removed by treatment with $\mathrm{SO}_{2} \cdot{ }^{19}$ The metal oxide buildups have been observed in all tile types, all valley soils, and in a11 locations in the valley. 18

The problem associated with Colorado River water lies in the seasonal variation of the salinity of the water. The lowest salinity level occurs in the summer when the most salt tolerant crops are grown; the lowest salt tolerant crops are grown when the salinity is at its peak in the autumn. 20 Colorado River water contains $1-1 / 4$ tons of soluble salts per acre-foot.

Salts injure plants by exerting an osmotic potential on the plant that requires additional energy for

\footnotetext{
* Reference to a company or product name does not imply approval or recommendation of the product by the University of California or the U.S. Energy Research \& Development Administration to the exclusion of others that may be suitable.
}

the plant to take water from the soil. This manifests itself in reduced growth and lower yields. 21 Figure 4.4 shows the salt effects on yield of major Imperial Valley crops. The figure is taken from Mayberry. 21

\section{Livestock Production}

Calves are imported from outside the state at a weight of 350 to 400 1bs. These are then raised for slaughter in feedlot operations in the valley. During their stay, they gain approximately 500 1bs. and are fed a diet of mill feed and roughage. The primary cause of livestock death is pneumonia. One quarter of all cattle arriving have shipping fever. Numerous infectious diseases are commonplace in valley feedlots because of contaminated pens and the cattle having varied and, presumably, contaminated origins. 22 Livestock and dairy statistics of 1973-1974 are shown in Table 4.7. ${ }^{1}$ Fig. 4.5 depicts livestock trends for 15 years. 23

Weed Control, Pest Control and Fertilizer

Table 4.8 1ists the weeds (grasses, sedges, and broadleaves) found in the Imperial Valley. All are common weeds and are found throughout Imperial County in the 


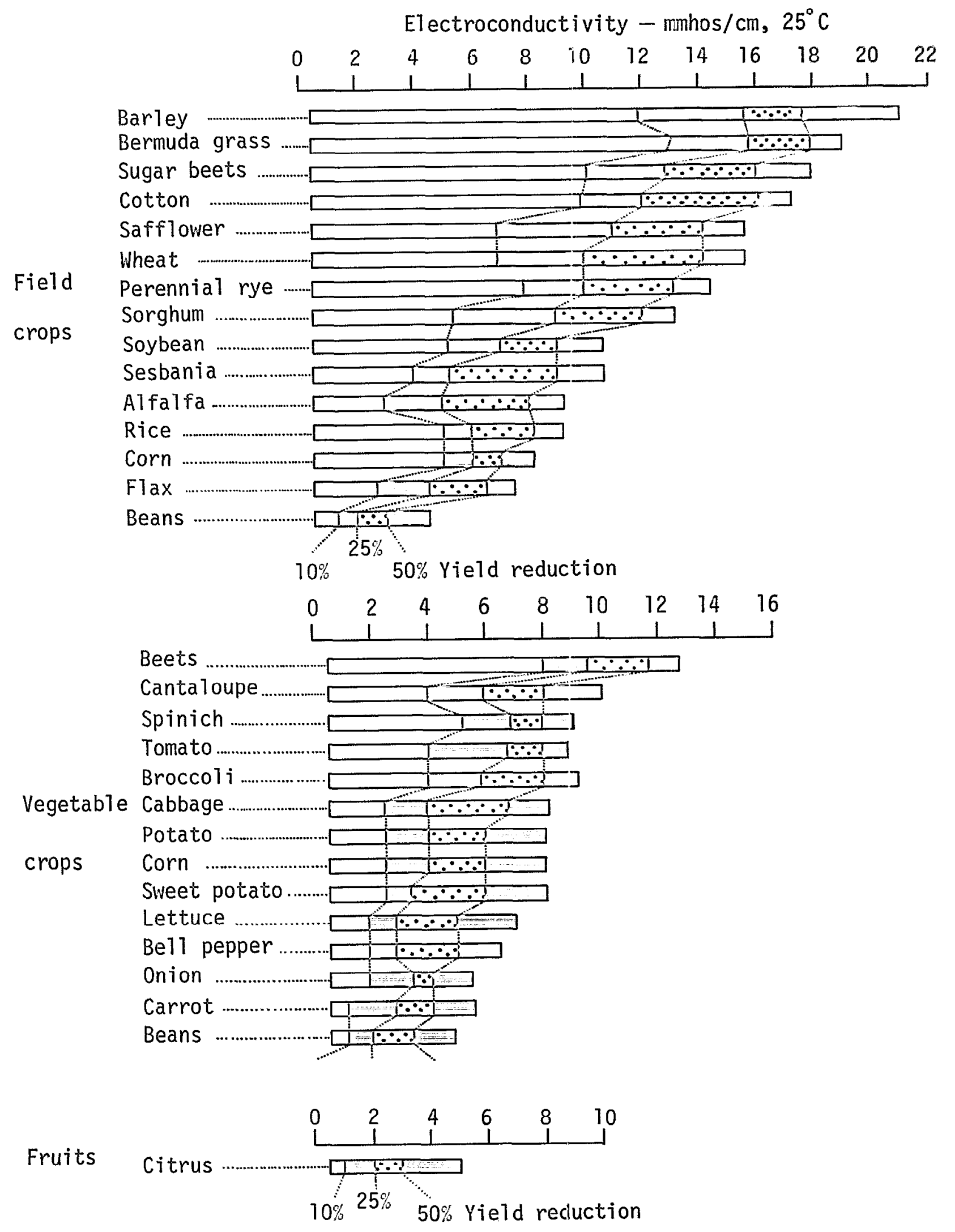

Fig. 4.4. Salt tolerance of crops. 21 The salt tolerances are given for the period of rapid plant growth and maturation. Crops are ranked in order of decreasing salt tolerance. Yield reductions are divided at 10, 25, and 50\% reduction. 
Table 4.7. Livestock and dairy products of the Imperial Valley. 17

\begin{tabular}{|c|c|c|c|c|c|c|c|}
\hline \multicolumn{8}{|c|}{ Livestock and dairy, 1973 to 1974} \\
\hline & Year & Head & Unit grain & Unit & Total & Per unit & Value \\
\hline Cattle $\ldots \ldots \ldots \ldots \ldots \ldots$ & $\begin{array}{l}1974 \\
1973\end{array}$ & $\begin{array}{l}720,000 \\
798,000\end{array}$ & $\begin{array}{l}4.97 \\
5.34\end{array}$ & $\begin{array}{l}\text { Cwt } \\
\text { Cwt }\end{array}$ & $\begin{array}{l}3,578,000 \\
4,261,000\end{array}$ & $\begin{array}{l}42.21 \\
43.76\end{array}$ & $\begin{array}{l}\$ 151,027,000 \\
186,461,000\end{array}$ \\
\hline Sheep $. . . \ldots \ldots \ldots \ldots . . . .$. & $\begin{array}{l}1974 \\
1973\end{array}$ & $\begin{array}{l}160,000 \\
160,000\end{array}$ & $\begin{array}{l}0.50 \\
0.50\end{array}$ & $\begin{array}{l}\text { Cwt } \\
\text { Cwt }\end{array}$ & $\begin{array}{l}80,000 \\
80,000\end{array}$ & $\begin{array}{l}38.50 \\
35.91\end{array}$ & $\begin{array}{l}3,080,000 \\
2,873,000\end{array}$ \\
\hline Wool $\ldots \ldots \ldots \ldots \ldots \ldots \ldots$ & $\begin{array}{l}1974 \\
1973\end{array}$ & $\begin{array}{l}162,000 \\
180,000\end{array}$ & $\begin{array}{l}4.0 \\
5.10\end{array}$ & $\begin{array}{l}\text { Lbs. } \\
\text { Lbs. }\end{array}$ & $\begin{array}{l}648,000 \\
918,000\end{array}$ & $\begin{array}{l}0.40 \\
0.75\end{array}$ & $\begin{array}{l}259,000 \\
689,000\end{array}$ \\
\hline Milk $\ldots \ldots \ldots \ldots \ldots \ldots \ldots$ & $\begin{array}{l}1974 \\
1973\end{array}$ & & & $\begin{array}{l}\text { Cwt } \\
\text { Cwt }\end{array}$ & $\begin{array}{l}94,900 \\
88,700\end{array}$ & $\begin{array}{l}8.30 \\
6.75\end{array}$ & $\begin{array}{l}788,000 \\
599,000\end{array}$ \\
\hline MIscellaneous Livestock..... & $\begin{array}{l}1974 \\
1973\end{array}$ & & & & & & $\begin{array}{l}28,000 \\
22,000\end{array}$ \\
\hline & & & & $\begin{array}{l}\text { Total } \\
\text { Total }\end{array}$ & $\begin{array}{l}1974 \\
1973\end{array}$ & & $\begin{array}{r}\$ 155,182,000 \\
190,644,000\end{array}$ \\
\hline
\end{tabular}


Table 4-8. Effectiveness of control of common weeds by herbicides in the Imperial Valley.

\begin{tabular}{|c|c|c|c|c|c|c|c|c|c|c|c|c|c|c|c|c|c|c|c|c|c|c|c|c|c|c|c|}
\hline & & & & hort & Esidua & $1 \mathrm{ti}$ & ime ( & $<2 \pi$ & onth & & & & & & $\begin{array}{r}\text { dium } \\
(2\end{array}$ & $\begin{array}{l}\text { resid } \\
-6 \text { nor }\end{array}$ & $\begin{array}{l}\text { waI } \\
\text { thss) }\end{array}$ & time & & & ong & residual & tims & $e(>$ & 6 mo & nths & \\
\hline & 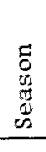 & $\vec{E}$ & 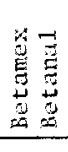 & 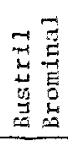 & 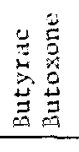 & 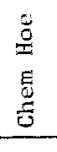 & 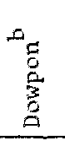 & 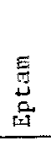 & 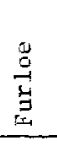 & 總 & 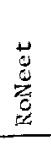 & 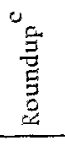 & $\begin{array}{l}\hat{I} \\
\hat{N} \\
\hat{N}\end{array}$ & $\begin{array}{l}4 \\
\frac{0}{0} \\
\frac{0}{0} \\
\end{array}$ & 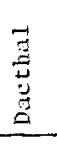 & 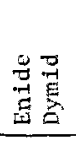 & $\begin{array}{l}\text { w } \\
0 \\
0 \\
0 \\
\end{array}$ & 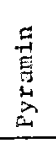 & $\begin{array}{l}\ddot{2} \\
0 \\
F\end{array}$ & 晜 & 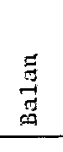 & 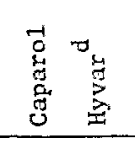 & 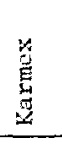 & ह્己 & 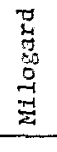 & 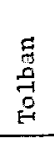 & 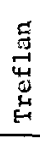 \\
\hline Grasses and sedge $e$ & & & & & & & & & & & & & & & & & & & & & & & & & & & \\
\hline Barnyard grass & $s$ & 0 & 0 & 0 & 0 & 0 & 1. & 3 & 1 & 3 & 3 & & 0 & 3 & 3 & 3 & 2 & 0 & 3 & 3 & 3 & 2 & 2 & 2 & 3 & 3 & 3 \\
\hline Bermuda grass $\hat{f}$ & $s$ & 0 & 0 & 0 & 0 & 0 & 2 & 1 & 0 & 0 & 0 & & 0 & 0 & 0 & 0 & 0 & 0 & & 0 & & 0 & 0 & 0 & 0 & 0 & 1 \\
\hline Littleseed canary grass & $v$ & 0 & 0 & 0 & 0 & 3 & 0 & 3 & 3 & & 3 & & 0 & & 3 & 3 & 3 & 0 & 3 & 3 & 1 & 3 & & 3 & & $i$ & 1 \\
\hline Mexican sprangletop & $\mathrm{s}$ & 0 & 0 & 0 & 0 & 0 & 1 & 2 & 0 & 2 & 3 & & 0 & 3 & 2 & & 0 & 0 & 2 & 2 & 3 & 0 & 0 & 1 & 2 & 3 & 3 \\
\hline Purple nutgrass $f$ & s & 0 & 0 & 0 & 0 & 0 & 0 & 2 & 0 & 3 & 1 & & 0 & 0 & 0 & 0 & 0 & 0 & 1 & 1 & & 0 & 1 & 0 & 1 & 0 & 0 \\
\hline Rabbitfoot grass & $\mathrm{H}$ & 0 & 0 & 0 & 0 & 3 & 0 & 3 & 3 & & 3 & & 0 & & 3 & 3 & 3 & 0 & 3 & & 3 & & 1 & 3 & & 1 & \\
\hline Sandbur & $\mathrm{s}$ & 0 & 0 & 0 & 0 & 0 & 0 & 2 & & 3 & 2 & & 0 & & 3 & & 2 & & & 2 & 3 & 1 & 2 & & 2 & & 3 \\
\hline Volunteer winter cereals & & 0 & 0 & 0 & 0 & 3 & 0 & 2 & 3 & & 3 & & 0 & & 3 & 3 & 3 & 0 & 1 & 3 & 1 & 3 & 1 & 3 & 3 & 1 & 1 \\
\hline Water grass & $\mathrm{s}$ & 0 & 0 & 0 & 0 & 0 & 1. & 3 & 1 & 3 & 3 & & 0 & 3 & 3 & 3 & 2 & 0 & 3 & 3 & 3 & 2 & 2 & 2 & 3 & 3 & 3 \\
\hline Vild oats & $\mathrm{V}$ & 0 & 0 & 0 & 0 & 3 & 0 & 3 & 3 & & 3 & & 0 & & 3 & 3 & 1 & 0 & 2 & 3 & 1 & & & 3 & 3 & 1 & 2 \\
\hline Xe11ow nutgrass ${ }^{f}$ & $s$ & 0 & 0 & 0 & 0 & 0 & 0 & 2 & 0 & 3 & 1 & & 0 & 0 & 0 & 0 & 0 & 0 & 1 & 1 & & 0 & 1 & 0 & 1 & 0 & 0 \\
\hline Broadleavese & & & & & & & & & & & & & & & & & & & & & & & & & & & \\
\hline Cheeseweeds & $\mathrm{H}$ & 3 & 2 & 3 & 1 & & 0 & 2 & 2 & & 2 & & 2 & & 2 & 2 & 2 & 2 & 3 & & 1 & 2 & 2 & 1 & 2 & & \\
\hline Curly dock $\mathrm{f}$ & $\mathrm{W}$ & 2 & 2 & 3 & 2 & & 0 & 2 & 2 & & 1) & & 3 & & & & & 1 & 1 & 3 & & & & 1 & & & \\
\hline Field bindweed & $s$ & 2 & & 0 & 1 & & $n$ & 0 & & & 0 & & 2 & & 0 & & 1 & 0 & & 0 & 0 & 1 & 0 & & 0 & & 1 \\
\hline Hiter weed (five hook bassia) & $\mathrm{s}$ & 3 & 3 & 3 & 3 & & 0 & 3 & 3 & & & & 3 & & 2 & & 2 & & & 3 & & 2 & 2 & 2 & 3 & & \\
\hline Inmbsquarters & $W$ & 3 & 3 & 3 & 3 & & 0 & 3 & 3 & & 2 & & 3 & 3 & 3 & 3 & 3 & 3 & 3 & 3 & 3 & & 3 & 3 & 3 & 2 & 2 \\
\hline London rocker & W & 3 & 3 & 3 & 3 & & 0 & 3 & 3 & & 1 & & 3 & & 1 & 0 & 3 & 3 & 3 & 3 & 0 & 3 & 3 & 3 & 3 & 1 & 1 \\
\hline Meralean morning glory & 5 & 3 & & & 3 & & 0 & & & & & & 3 & 2 & 0 & & 3 & & & 2 & & 3 & 3 & & 2 & 1 & 1 \\
\hline Wottleleat goosefoot & $W$ & 3 & 3 & 3 & 3 & & $a$ & 2 & 3 & & 2 & & 3 & 3 & 2 & 3 & 3 & 3 & 3 & 3 & 2 & 3 & 3 & 3 & 3 & 2 & 2 \\
\hline $\begin{array}{l}\text { Pigwecd } \\
\text { Prickly lettues: }\end{array}$ & S & 3 & $3^{h}$ & 3 & 3 & & 0 & 3 & 2 & & 0 & & 3 & 3 & 2 & & 2 & 3 & 3 & 3 & 2 & 3 & 2 & 3 & 3 & 3 & 3 \\
\hline $\begin{array}{l}\text { Prickly lettuces } \\
\text { Punctuce vine }\end{array}$ & W & 3 & 3 & 3 & 3 & & 0 & 2 & 3 & & 1 & & 3 & & 0 & 0 & 3 & 3 & 3 & 3 & 0 & 3 & 3 & 0 & & 0 & a \\
\hline $\begin{array}{l}\text { Puncture vine } \\
\text { Fus } 1 \text { 1 } 110\end{array}$ & 5 & 3 & & & 2 & & 0 & 3 & & 3 & & & 3 & & 0 & & 1 & 0 & 2 & 2 & & 2 & 1 & & 2 & & 2 \\
\hline & $\therefore$ & 3 & 7 & " & $\begin{array}{l}1 \\
3\end{array}$ & & $\begin{array}{l}0 \\
0\end{array}$ & $\begin{array}{l}3 \\
3\end{array}$ & 3 & 1 & 3 & & 3 & 3 & 3 & 3 & 3 & 3 & 3 & 3 & 3 & & 3 & 3 & 3 & 3 & 3 \\
\hline $\begin{array}{l}\text { Iussian thistle } \\
\text { Shepherds purtite }\end{array}$ & $\stackrel{s}{w}$ & $\begin{array}{l}3 \\
3\end{array}$ & 1 & 3 & 3 & & 0 & 3 & 3 & & 3 & & 3 & & & & 3 & & & 2 & & & $\ell$ & & 4 & & \\
\hline Silversherth linotweed & $\mathrm{W}$ & 3 & $i$ & 3 & 2 & & 1) & 2 & 2 & & $i$ & & 3 & & i & 1 & $\begin{array}{l}3 \\
3\end{array}$ & $\begin{array}{l}3 \\
2\end{array}$ & $\begin{array}{l}3 \\
2\end{array}$ & $\begin{array}{l}3 \\
1\end{array}$ & $\frac{1}{1}$ & $\begin{array}{l}3 \\
2\end{array}$ & $\begin{array}{l}3 \\
2\end{array}$ & $\begin{array}{l}3 \\
2\end{array}$ & 3 & & $\frac{1}{1}$ \\
\hline Sour clovey! & $\forall$ & 3 & 1 & 3 & $a$ & & 0 & 0 & 0 & & 0 & & 3 & & $\begin{array}{l}1 \\
0\end{array}$ & 3 & $\begin{array}{l}3 \\
1\end{array}$ & $\frac{2}{1}$ & 0 & 2 & 0 & $\begin{array}{l}2 \\
2\end{array}$ & $\begin{array}{l}2 \\
1\end{array}$ & $\begin{array}{l}2 \\
1\end{array}$ & & 0 & $\begin{array}{l}1 \\
0\end{array}$ \\
\hline Sowthiste & $\mathrm{H}$ & 3 & 3 & 3 & 3 & & 0 & 2 & 2 & & 1 & & 3 & & 0 & $a$ & 3 & $\hat{2}$ & 2 & 3 & 0 & 3 & 3 & 0 & & 0 & 0 \\
\hline Sumt lower & 5 & 3 & 3 & 3 & 3 & & 0 & 2 & 3 & & 0 & & 3 & 0 & 0 & 0 & 3 & 2 & 1 & 3 & 0 & 3 & 3 & & 3 & 0 & 0 \\
\hline White hor mett $10^{\circ}$ & $\mathrm{s}$ & 3 & & & $"$ & & 0 & $a$ & & & 0 & & 3 & & & 0 & & & & a & & 1 & 1 & & 1 & & 0 \\
\hline White crewet clower & :" & 3 & 1 & & 0 & & 0 & 0 & 0 & & 0 & & 3 & & 0 & & & 1 & 0 & 2 & 0 & 2 & 1 & & 2 & & 0 \\
\hline wild beet & $\because$ & 3 & 0 & 1 & 2 & & 0 & 1 & 2 & & 0 & & 3 & & 2 & & 3 & 0 & 1 & & 3 & & 1 & 3 & & & 3 \\
\hline Wright groudenery & 5 & 3 & 3 & 3 & 3 & & a & 3 & 1 & & $\mathrm{I}$ & & 3 & 1 & 1 & 0 & 3 & 3 & 3 & 1 & 0 & 3 & 3 & & 3 & 0 & 0 \\
\hline
\end{tabular}

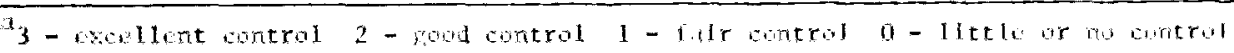

"controls anly proses

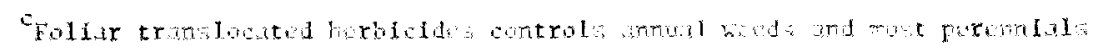

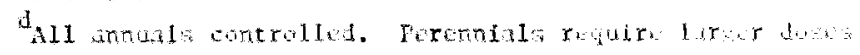

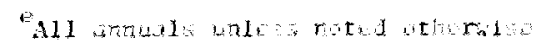

$t_{\text {Faremial }}$

Brmul ar biemi 1

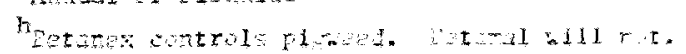


irrigated areas. This table was constructed from information taken from Cudney. 24 In Table 4.8, the herbicides used in the valley are listed, grouped according to their persistence in the environment. Herbicides applied to the most common crops are 1isted in Table 4.9. ${ }^{24}$ By comparing these two tables, one can infer the effectiveness of a herbicide in weed control.

Table 4.10 contains the important pests in the Imperial Valley and the crops that they damage. Pests are classified as serious and as possible or occasional pests. If no such information is given then the pest is simply deemed pest. This information is taken from Cudney et al. 25 Kontaxis, ${ }^{26}$ Sharma, ${ }^{27}$ and Meister. ${ }^{28}$ In Table $4.11,29$ the total amounts of fertilizers sold in Imperial County in 1975 are listed. In Table 4.12, the amounts of all pesticides and herbicides used in Imperial County in 1975 are shown. These are broken down into pounds used for each crop grown or other use. The total use of the most important pesticides and herbicides for the years 1970, 1971, 1972, 1974, and 1975 is given in Table 4.13. The data in Tables 4.12 and 4.13 were obtained through the courtesy of Ming-Yu Li. 30
4.2 THE ECOLOGY OF THE SALTON SEA QUASI-MARINE ECOSYSTEM

The Salton Sea, shown on the map in Fig. 4.1, is classified by Hedgepeth ${ }^{31}$ and Whitney ${ }^{32}$ as a saltern or inland brine. Table $4.14^{33,34,35}$ contains a summary and history of the chemical characteristics of the lake. Irrigation has provided the source of inflow water for the sea since 1907. Between 1960 and 1967, evaporation from the sea averaged 1,330,000 acre-feet (af) annually. 34 Inflow to the sea is about 1,250,000 af from irrigation runoff, 50,000 af from subsurface flow, and 40,000 af from precipitation. $^{34}$ The Alamo River from 1960 to 1967 contributed an average of 637,000 af annually, and the New River an average of 413,000 af. Currently the sea is rising. From 1907 to the 1930 's, the Alamo channel brought water from the Colorado to Imperial Valley. After the completion of Hoover Dam, the Alamo channel was replaced by the A11 American Canal. 36 This is still the current means of importation of Colorado water to the valley. In going through the irri fation and drainage system the chrmical composition of the water changes, as seen in Table $4.15^{5}$

When the sea was first formed, salinity increased sharply by the 
Table 4.9. Crop herbicides most commonly used in Imperial Vallcy. ${ }^{a, b}$

Alfalfa

Eptam, Balan, IPC (Chem Hoe), 2,4-DB, Kerb

Asparagus

Karmex, Princip, Banvel, Lorox

Carrots

Treflan, Lorox, Carrot Oil

Cereals

2,4-D, Buctril, Brominal, Banvel

Cole crops

Tok, Dactha1, Prefar

Cotton

Dactha1, Caparo1, Treflan, Cobex, Tolban, Cotoran, MSMA

\section{Lettuce}

Balan, Kerb, IPC (Chem Hoe), Prefar

Melons

Prefar, Dacthal

\section{Onions}

Dacthal, Sulfuric Acid, Dow Selective, Tok, CIPC (Furloe)

\section{Sorghum}

Aatrex, Milogard, Igran, Banvel

$\underline{\text { Sugar beets }}$

Ro Neet, Tok, IPC (Chem Hoe), Eptam, Betanal Betanex, Pyramin Plus, Fur1oe

Tomatoes

Devrinol, Enide, Dymid, Trefmid, Prefar, Dactha1, Tillam

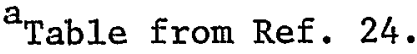

${ }^{b}$ AIl items are registered trademarks except IPC, CIPC, MSHA, Ro Neet, 2,4-D, sulfuric acid, and carrot oil. 
Table 4-10. Fests ${ }^{a}$ of Imperial Valley

Small grains

ins

(barley, oats

wheat, rye) Sorghum Sugar beets Tomatoes Watermelon

s

In

$\mathrm{m}$

m

$m p$

$\mathrm{m}$ $\mathrm{p}$

$\mathbf{s}$

s

s

$s(p)$

m

m

s

p

m

$p(m)$

m

m

m

s

s

s

s

s

P

$s$

p

s

s

$-53-$ 


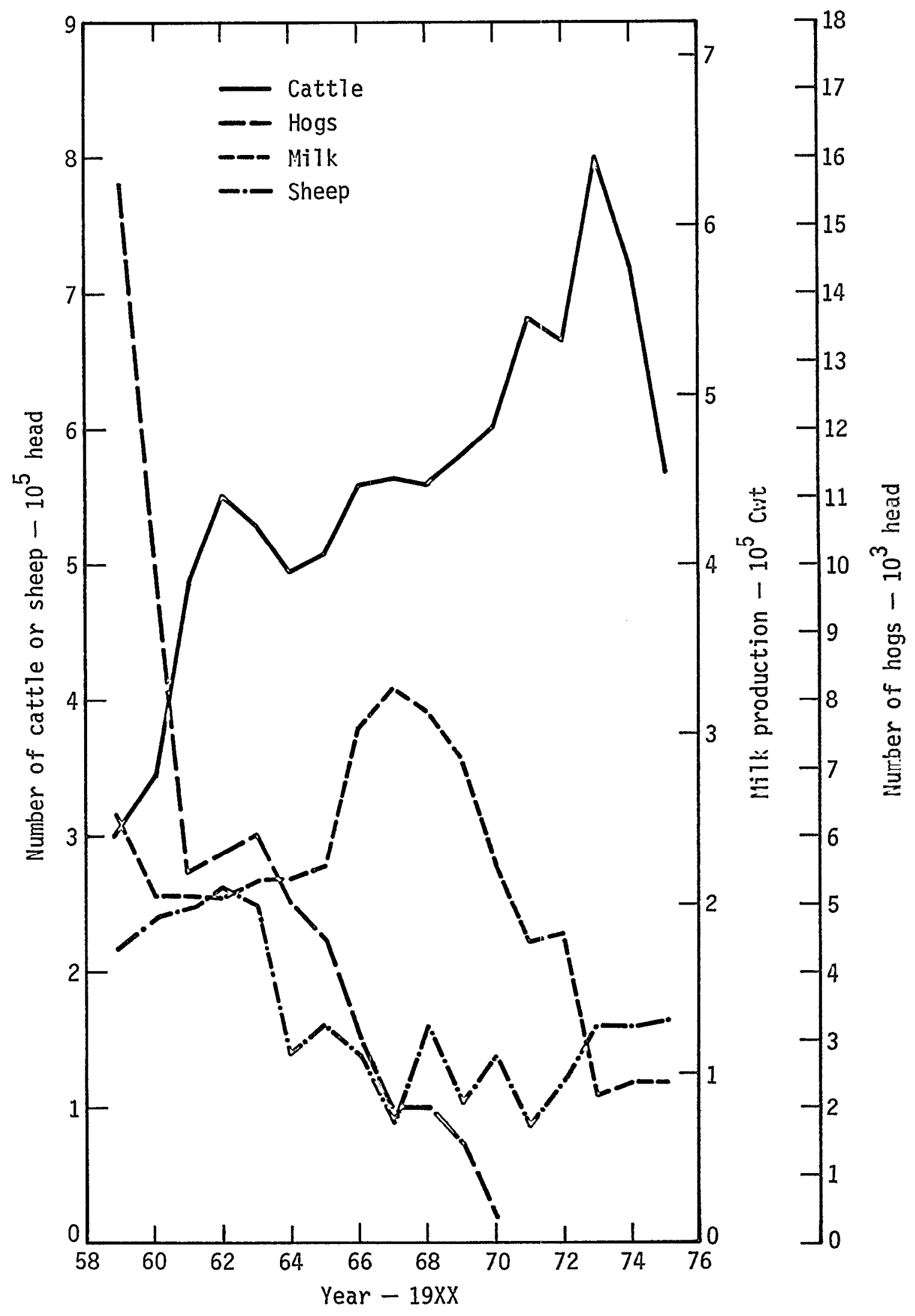

Fig. 4.5. Livestock and dairy statistics for Imperial County; annual livestock turnover and milk production for Imperial County from 1959 to 1975.23 
Table 4.11. Tonnage of fertilizers and minerals sold in Imperial County in $1975 .^{a}$

\begin{tabular}{|c|c|c|c|c|c|}
\hline Material & Analysis & $\begin{array}{l}\text { Material, } \\
\text { total tons }\end{array}$ & Dry & Liquid & $\begin{array}{l}\text { Analysis, } \\
\text { total tons }\end{array}$ \\
\hline & $N$ & & & & 67,578 \\
\hline \multirow[t]{2}{*}{ Fertilizer } & $\mathrm{P}_{2} \mathrm{O}_{5}$ & 240,335 & 87,361 & 152,473 & 30,406 \\
\hline & $\mathrm{K}_{2} \mathrm{O}$ & & & & 855 \\
\hline Gypsum & $\mathrm{CaSO}_{4}$ & 47 & 47 & -- & 39 \\
\hline Iron oxide & $\mathrm{Fe}$ & 182 & 182 & -- & 36 \\
\hline $\begin{array}{l}\text { Lime-sulfur } \\
\text { solution }\end{array}$ & $\begin{array}{l}\text { Calcium } \\
\text { polysulfide }\end{array}$ & 4,743 & $\cdots$ & 4,743 & 1,163 \\
\hline Soil sulfur & $S$ & 902 & 902 & --- & 844 \\
\hline Dolomite & $\mathrm{CaCO}_{3}, \mathrm{Mg}$ & 2 & 2 & -- & --- \\
\hline Limes tone & $\mathrm{CaCO}_{3}$ & 106 & 106 & -- & 95 \\
\hline Zinc oxide & $\mathrm{Zn}$ & 9 & 9 & --- & 6 \\
\hline Al1 other & $-\infty$ & 2,860 & 2,860 & --- & --- \\
\hline
\end{tabular}

${ }^{a}$ Data from California Department of Food and Agriculture.

dissolution of sea bed deposits of salt directly into the water. The sea's salinity is now gradually approaching $40,000 \mathrm{ppm}$ with about 5.6 million tons of salt accumulating each year. 34

\section{History of Ecosystem}

All fish species in the Salton Sea have been introduced either deliberately or accidentally by man. The first set of fish in the lake were freshwater species. These were carp Cyprinus carpio, striped mullet Mugil cephalus, humpback sucker Xyranchen texanus, bonytail Gila robusta, and rainbow trout
Salmo gairdneri. These species were common from 1916 to 1929. The carp and bonytail disappeared, and the mullet was scarce, and trout existed only near the inlets by 1929. By 1952 there were only four species of fish in the lake. ${ }^{32}$

Attempts have been made to introduce 29 invertebrate species. These were introduced to establish a food base for the fish. By 1967 there were eight fish species and seven invertebrate taxa. These are listed in Table 4.16. This history is reviewed by Whitney. 32

oglesby 37 has observed another copepod species (unidentified 
Table 4.12. Pounds of pesticides and herbicide:s applied in Imperial Valley in 1975. a

\begin{tabular}{|c|c|c|c|c|c|c|c|c|}
\hline Uses & Abate & $\begin{array}{l}\text { Acephate } \\
\text { (orthene) }\end{array}$ & Aldicarb & Aldrin & irtitrole & Ammonia & $\begin{array}{l}\text { Aromatic } \\
\text { purroleunt } \\
\text { solvont: }\end{array}$ & Atrolsot \\
\hline Alfalfa & - & - & - & - & - & - & 8,833 & - \\
\hline Asparagus & - & - & - & - & - & - & 24 & - \\
\hline Barley & - & - & - & - & - & - & 30.6 & - \\
\hline Cabbaype & - & - & - & - & - & - & 32.8 & - \\
\hline Carrot & - & - & - & - & - & - & 199 & - \\
\hline Caulif lower & - & - & - & - & - & - & 41.9 & - \\
\hline Citrus & - & - & - & - & - & - & - & - \\
\hline Gorn & - & - & - & - & - & - & - & - \\
\hline Cotton & - & - & 27,870 & - & - & - & $10 \%, 329$ & - \\
\hline Cucumber & - & - & - & - & - & - & $7 \varepsilon$ & - \\
\hline $\begin{array}{l}\text { Fallow } \\
\text { farmland }\end{array}$ & - & - & - & - & - & - & - & - \\
\hline Figs & - & - & - & - & - & - & - & - \\
\hline $\begin{array}{l}\text { Forage, hay, } \\
\text { and silage }\end{array}$ & - & - & - & - & - & - & 5.5 & - \\
\hline Garlic & - & - & - & - & - & - & - & - \\
\hline $\begin{array}{l}\text { Grasses, } \\
\text { grains, and } \\
\text { fibur crops }\end{array}$ & - & - & - & - & - & - & - & \\
\hline Leettuce (head) & - & 774 & - & - & - & $\therefore .4$ & 17.22 & - \\
\hline Lettuce (lear) & - & - & - & - & - & - & $\Delta$. & - \\
\hline Melons & - & - & - & - & - & - & $\because 72.7$ & - \\
\hline $\begin{array}{l}\text { Miscellaneous } \\
\text { vegetables }\end{array}$ & - & - & - & - & - & - & $\therefore, 32 \pi$ & - \\
\hline Nectarines & - & - & - & - & - & - & 11.9 & - \\
\hline Oats & - & - & - & - & - & - & - & - \\
\hline Onions & - & - & - & - & - & - & 3,160 & - \\
\hline Ornamentals & - & - & - & - & - & - & - & - \\
\hline other & - & - & - & 880 & 317 & - & 234 & $\therefore(10)$ \\
\hline Safflower & - & - & - & - & - & - & - & - \\
\hline Sorghum & - & - & - & - & - & - & 479 & 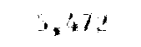 \\
\hline Squash & - & - & - & - & - & - & $6 i$ & - \\
\hline Sugar beet & - & - & 12,135 & - & - & - & 11,002 & - \\
\hline Tomato & - & - & - & - & - & - & 3,020 & - \\
\hline Turf & - & - & 618 & - & - & - & 544 & - \\
\hline Turnip & - & - & - & - & - & & 0.9 & - \\
\hline Vector control & 0.36 & - & - & - & - & - & - & - \\
\hline Wheat & - & - & - & - & - & - & 1,588 & - \\
\hline Total. & 0.36 & 774 & 40,623 & 880 & 317 & 2.4 & 162,499 & 3,075 \\
\hline
\end{tabular}

adata from Ming-Yu Li, Environmental Toxicology Center, University of California, Davis. 
Table 4.12. Pounds of pesticides and herbicides applied in Imperial Valley in 1975 . $^{a}$

\begin{tabular}{|c|c|c|c|c|c|c|c|c|}
\hline Uses & $\begin{array}{l}\text { Atrazine, } \\
\text { other } \\
\text { related }\end{array}$ & $\begin{array}{l}\text { Avitrol } \\
200\end{array}$ & Azodrin & Balan & Baygon & Benomy 1 & Betasan & Bidrin \\
\hline Alfalfa & - & - & - & 3,997 & - & - & - & 384 \\
\hline Asparagus & - & - & - & - & - & - & & - \\
\hline Barley & - & - & - & - & - & - & - & - \\
\hline Cabbage & - & - & - & - & - & - & - & - \\
\hline Carrot & - & - & - & - & - & - & - & - \\
\hline Cauliflower & - & - & - & - & - & - & - & - \\
\hline Citrus & - & - & - & - & - & - & - & - \\
\hline Corn & - & - & - & - & - & - & - & - \\
\hline Cotton & - & - & 105,399 & - & - & - & - & 6,902 \\
\hline Cucumber & - & - & - & - & - & - & - & - \\
\hline $\begin{array}{l}\text { Fallow } \\
\text { farmland }\end{array}$ & - & - & - & - & - & - & - & - \\
\hline Figs & - & - & - & - & - & - & - & - \\
\hline $\begin{array}{l}\text { Forage, hay, } \\
\text { and silage }\end{array}$ & - & - & - & - & - & - & - & - \\
\hline GarIfc & - & - & - & - & - & - & - & - \\
\hline $\begin{array}{l}\text { Grasses, } \\
\text { grains, and } \\
\text { fiber crops }\end{array}$ & - & - & - & - & - & - & - & - \\
\hline Lettuce (head) & - & - & - & 11,207 & - & - & 300 & - \\
\hline Lettuce (Ieaf) & - & - & - & - & - & - & 204 & - \\
\hline Melons & - & - & - & - & - & 210 & 530 & - \\
\hline $\begin{array}{c}\text { Miscellaneous } \\
\text { vegetables }\end{array}$ & - & - & - & - & - & - & - & - \\
\hline Nectarines & - & - & - & - & - & - & - & - \\
\hline Oats & - & - & - & - & - & - & - & - \\
\hline Onions & - & - & - & - & - & - & - & - \\
\hline Ornamentals & - & - & - & - & - & - & - & - \\
\hline Other & 137 & 0.1 & - & - & 110 & - & - & - \\
\hline Safflower & - & - & - & - & - & - & - & - \\
\hline Sorghum & 288 & - & - & - & - & - & - & - \\
\hline Squash & - & - & - & - & - & 21 & - & - \\
\hline Sugar beet & - & - & - & - & - & - & - & - \\
\hline Tomato & - & - & - & - & - & - & 2,372 & - \\
\hline Turf & - & - & - & - & - & - & - & - \\
\hline Turnip & - & - & - & - & - & - & - & - \\
\hline Vector control & - & - & - & - & - & - & - & - \\
\hline Wheat & - & - & - & - & - & - & - & - \\
\hline Tota1 & 425 & 0.1 & 105,399 & 15,204 & 110 & 231 & 3,406 & 7,286 \\
\hline
\end{tabular}

${ }^{9}$ Data from Ming-Yu Li, Environmental Toxicology Center, University of California, Davis. 
Table 4.12. Pounds of pesticides and herbicides applied in Imperial Valley in $1975 .^{a}$

\begin{tabular}{|c|c|c|c|c|c|c|c|c|}
\hline Uses & $\begin{array}{l}\text { Bromoxynil } \\
\text { octanoate }\end{array}$ & BTB & $\begin{array}{l}\text { N-see- } \\
\text { Euty1-4- } \\
\text { tert-buty } 1 \\
2,6-d \text { initro } \\
\text { aniline }\end{array}$ & $\begin{array}{l}\text { Cacodylic } \\
\text { acid }\end{array}$ & cacbary 1 & Carbofurin & $\begin{array}{c}\text { Curballe } \\
\text { at } 1 \text { a }\end{array}$ & 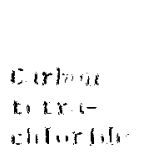 \\
\hline Alfalfa & - & 119 & - & - & $\div, 696$ & 16,771 & $\therefore 1$ & - \\
\hline Asparagus & - & - & - & - & 56 & - & - & - \\
\hline Barley & - & - & - & - & - & - & - & - \\
\hline Cabbage & - & 31.6 & - & - & - & - & - & - \\
\hline Carrot & - & - & - & - & 520 & - & - & - \\
\hline Cauliflower & - & - & - & - & 50 & - & - & - \\
\hline Citrus & - & - & - & - & 88 & - & - & - \\
\hline Corn & - & - & - & - & 58 & - & - & - \\
\hline Cotton & - & 78 & 320 & 1,391 & 13,138 & - & - & - \\
\hline Cucumber & - & - & - & - & 32.5 & - & - & - \\
\hline $\begin{array}{l}\text { Fallow } \\
\text { farmland }\end{array}$ & - & - & - & - & - & - & - & - \\
\hline Figs: & - & - & - & - & - & - & - & - \\
\hline $\begin{array}{l}\text { Forage, hay, } \\
\text { and silage }\end{array}$ & - & - & - & - & - & - & - & - \\
\hline Garli: & - & - & - & - & - & - & - & - \\
\hline $\begin{array}{l}\text { Grasses, } \\
\text { grains, and } \\
\text { fiber crops }\end{array}$ & - & - & - & - & - & - & - & - \\
\hline Lettuce (head) & - & 2,103 & - & - & 2,356 & - & 1 & - \\
\hline Lettuce (leaf) & - & 9.9 & - & - & - & - & - & - \\
\hline Melons & - & 96 & - & - & 3,0194 & $\sim$ & - & - \\
\hline $\begin{array}{c}\text { Micollancous } \\
\text { vepetables }\end{array}$ & - & 16.9 & - & - & 1411 & - & - & - \\
\hline Nectarines & - & - & - & - & - & - & - & - \\
\hline Oatsi & - & - & - & - & - & - & - & - \\
\hline Onion: & - & - & - & - & - & - & - & - \\
\hline Ornamentils & - & - & - & - & - & - & - & - \\
\hline nther & - & - & - & 13.45 & 207.2 & - & - & 171 \\
\hline Safflower & - & - & - & - & - & - & - & - \\
\hline Sorghum & - & - & - & - & 1,037 & - & - & - \\
\hline Sqquash & - & - & - & - & 158 & - & - & - \\
\hline sugar beet & - & - & - & - & 21,87 & - & $2+5$ & - \\
\hline lomato & - & 1.9 & - & - & 118 & - & - & - \\
\hline Turi & 11.6 & - & - & - & 1,007 & - & - & - \\
\hline Turnip & - & 0.64 & - & - & - & - & - & - \\
\hline Vectar control & - & - & - & - & - & - & - & - \\
\hline Wheat & 17,554 & - & - & - & - & - & - & - \\
\hline Total & 17,566 & 2,458 & 320 & $1,40 k$ & 34,974 & 16,770 & 162 & 477 \\
\hline
\end{tabular}

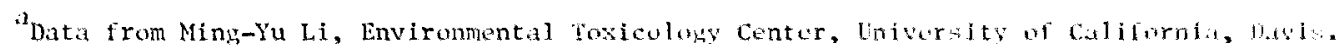



Table 4.12. Pounds of pesticides and herbicides applied in Imperial Valley in

\begin{tabular}{|c|c|c|c|c|c|c|c|c|}
\hline Uses & ChIordane & $\begin{array}{l}\text { Chlordane, } \\
\text { other } \\
\text { related }\end{array}$ & $\begin{array}{l}\text { Chloro- } \\
\text { picrin }\end{array}$ & CIPC & Cobex & $\begin{array}{l}\text { Copper } \\
\text { dihydrazi- } \\
\text { nium } \\
\text { sulfate }\end{array}$ & $\begin{array}{l}\text { Gopper } \\
\text { oxychloride } \\
\text { sulfate }\end{array}$ & Cryolite \\
\hline Alfalfa & - & - & - & - & - & - & - & - \\
\hline Asparagus & 244 & 1.63 & - & - & - & - & - & - \\
\hline Barley & - & - & - & - & - & - & - & - \\
\hline Cabbage & 9.6 & 6.4 & - & - & - & - & - & 540 \\
\hline Carrot & - & - & 960 & 280 & - & - & - & - \\
\hline Cauliflower & - & - & - & - & - & - & - & 1,425 \\
\hline Citrus & - & - & - & - & - & - & 94.6 & - \\
\hline Corn & - & - & - & - & - & - & - & - \\
\hline Cotton & - & - & - & - & 3,147 & - & - & - \\
\hline Cucumber & - & - & - & - & - & - & - & 3,997 \\
\hline $\begin{array}{l}\text { Fallow } \\
\text { farmland }\end{array}$ & - & - & - & - & - & - & - & - \\
\hline Figs & - & - & - & - & - & - & $=$ & - \\
\hline $\begin{array}{l}\text { Forage, hay, } \\
\text { and silage }\end{array}$ & - & - & - & - & - & - & - & - \\
\hline Garlic & - & - & - & - & - & - & - & - \\
\hline $\begin{array}{l}\text { Grasses, } \\
\text { grains, and } \\
\text { fiber crops }\end{array}$ & - & - & - & - & - & - & - & - \\
\hline Lettuce (head) & 746 & 497 & - & - & - & - & - & 163,572 \\
\hline Lettuce (leaf) & - & - & - & - & - & - & - & - \\
\hline Melons & - & - & 1,684 & - & - & - & - & 257,596 \\
\hline $\begin{array}{l}\text { Miscellaneous } \\
\text { vegetables }\end{array}$ & - & - & - & - & - & - & - & 5,375 \\
\hline Nectarines & - & - & - & - & - & - & - & - \\
\hline Oats & - & - & - & - & - & - & - & - \\
\hline Onions & 50.4 & 33.6 & 4,008 & 440 & - & - & - & - \\
\hline Ornamentals & - & - & - & - & - & - & - & - \\
\hline Other & 771.15 & 1.1 & 3.57 & - & - & - & - & - \\
\hline Safflower & - & - & - & - & - & - & - & - \\
\hline Sorghum & - & - & - & - & - & - & - & - \\
\hline Squash & - & - & - & - & - & - & - & 1,395 \\
\hline Sugar beet & - & - & 600 & - & - & - & - & - \\
\hline Tomato & - & - & 300 & - & - & 17.3 & - & 1,265 \\
\hline Turf & - & - & - & - & - & - & - & - \\
\hline Turnip & - & - & - & - & - & - & - & - \\
\hline Vector control & - & - & - & - & - & - & - & - \\
\hline Wheat & - & - & - & - & - & 41 & - & - \\
\hline Total & 1,821 & 701 & 7,556 & 720 & 3,147 & 58.3 & $94.6^{\circ}$ & 435,165 \\
\hline
\end{tabular}

${ }^{a}$ Data from Ming-Yu Li, Environmental Toxicology Center, University of California, Davis. 
Table 4.12. Pounds of pesticides and herbicides applied in Imperial Valley in 1975. ${ }^{\mathrm{a}}$

\begin{tabular}{|c|c|c|c|c|c|c|c|c|}
\hline Uses & Cypromid & $2,4-D$ & $\begin{array}{l}2,4-D, \\
\text { alkanol- } \\
\text { amine } \\
\text { salt }\end{array}$ & $\begin{array}{l}2,4-D, \\
\text { dimethyl- } \\
\text { amine } \\
\text { salt }\end{array}$ & $\begin{array}{l}2,4-D, \\
\text { dc jecyl- } \\
\text { anine } \\
\text { stit }\end{array}$ & $\begin{array}{l}2,4-D, \\
\text { sodium } \\
\text { salt }\end{array}$ & $\begin{array}{l}2,4-\mathrm{D}, \\
\text { tetradecy } 1- \\
\text { anfris: } \\
\text { ath }\end{array}$ & Dectinat \\
\hline Alfalfa & - & 289 & - & - & - & - & - & - \\
\hline A:sparagu: & - & - & - & 196 & - & 2,273 & - & - \\
\hline Barley & - & - & 538 & 672 & - & - & - & - \\
\hline Cabbage & - & - & - & - & - & - & - & $1,6+t_{6}$ \\
\hline Carrot: & - & - & - & - & - & - & - & - \\
\hline Cauliflower & - & - & - & - & - & - & - & $33 x$ \\
\hline Citru: & - & - & - & - & - & - & - & - \\
\hline Corn & - & - & - & - & - & - & - & - \\
\hline Cotton & - & - & - & - & - & - & - & $22_{4}^{\prime}, x_{1}^{\prime}$ \\
\hline Cucumber & - & - & - & - & - & - & - & - \\
\hline $\begin{array}{l}\text { Fallow } \\
\text { farmland }\end{array}$ & - & - & - & - & - & - & - & - \\
\hline Eigs & - & - & - & - & - & - & - & - \\
\hline $\begin{array}{l}\text { Forage, hay, } \\
\text { and silage }\end{array}$ & - & - & - & - & - & - & - & - \\
\hline Garlic & - & - & - & - & - & - & $\simeq$ & $2,6+4 i$ \\
\hline $\begin{array}{l}\text { Grasses, } \\
\text { gtains, and } \\
\text { fiber crops }\end{array}$ & - & - & - & - & - & - & - & - \\
\hline T.ettuce (head) & - & - & - & - & - & - & - & - \\
\hline Lettuce (leaf) & - & - & - & - & - & - & - & - \\
\hline Melon: & - & - & - & - & - & - & - & $3,6,71$ \\
\hline $\begin{array}{c}\text { Miscellaneous } \\
\text { vegetahles }\end{array}$ & - & - & - & - & - & - & - & 9.954 \\
\hline Nectarines & - & - & - & - & - & - & - & - \\
\hline Vata & - & - & - & - & - & - & - & - \\
\hline Unions: & - & - & - & - & - & - & - & $38,5,4$ \\
\hline urnamentals & - & - & - & - & - & - & - & - \\
\hline other & - & - & 25,221 & 142.6 & 1,021 & - & $25 \mathrm{th}$ & - \\
\hline Safflower & - & - & - & - & - & - & - & - \\
\hline sorghum & - & - & 57 & - & - & - & - & - \\
\hline Squash & - & - & - & - & - & - & - & $1, \cdots$ \\
\hline Sugar beet & - & - & - & - & - & - & - & - \\
\hline Tomato & 340 & - & - & - & - & - & - & 111,311 \\
\hline 'Turf & - & - & 142 & 171 & - & - & - & - \\
\hline Turnip & - & - & - & - & - & - & - & 1,114 \\
\hline Vector control & - & - & - & - & - & - & - & - \\
\hline Wheat & - & 616 & 33,585 & 13,179 & - & - & - & - \\
\hline Total & 340 & 905 & 59,543 & 14,361 & 1,021 & 2,273 & $25 b$ & $\sin _{2} k_{1} f_{2} y$ \\
\hline
\end{tabular}

a Data from Ming-Yu Li, Environmental Toxicology Center, Universicy of California, Davis: 
Table 4.12. Pounds of pesticides and herbicides applied in Imperial Valley in $1975 .^{a}$

\begin{tabular}{|c|c|c|c|c|c|c|c|c|}
\hline Uses & $\begin{array}{l}\text { Dalapon, } \\
\text { sodium } \\
\text { salt }\end{array}$ & $\begin{array}{l}4(2,4-D B) \\
\text { dimethyl- } \\
\text { amine } \\
\text { salt }\end{array}$ & $\mathrm{DBCP}$ & $\begin{array}{l}\text { DBCP, } \\
\text { other } \\
\text { related }\end{array}$ & $\begin{array}{c}D-D \\
\text { mixture }\end{array}$ & DDVP & $\begin{array}{l}\text { DDVP, } \\
\text { other } \\
\text { related }\end{array}$ & $\begin{array}{c}\text { 1-Decanol } \\
\text { or } \\
\text { N-decanol }\end{array}$ \\
\hline Alfalfa & - & 16,669 & - & - & - & - & - & - \\
\hline Asparagus & - & - & - & - & - & - & - & - \\
\hline Barley & - & - & - & - & - & - & - & - \\
\hline Cabbage & - & - & - & - & - & - & - & - \\
\hline Carrot & - & - & 828 & 43.6 & 38,392 & - & - & - \\
\hline Cauliflower & - & - & - & - & - & - & - & - \\
\hline Citrus & - & - & - & - & - & - & - & - \\
\hline Corn & - & - & - & - & - & - & - & - \\
\hline Cotton & 1,647 & - & 2,794 & - & - & - & - & - \\
\hline Cucumber & - & - & - & - & - & - & - & - \\
\hline $\begin{array}{l}\text { Fallow } \\
\text { farmland }\end{array}$ & - & - & - & - & - & - & - & - \\
\hline Figs & - & - & - & - & - & - & - & - \\
\hline $\begin{array}{l}\text { Forage, hay, } \\
\text { and silage }\end{array}$ & - & - & - & - & - & - & - & - \\
\hline Garlic & - & - & - & - & - & - & - & - \\
\hline $\begin{array}{l}\text { Grasses, } \\
\text { grains, and } \\
\text { fiber crops }\end{array}$ & - & - & - & - & - & - & - & - \\
\hline Lettuce (head) & - & - & - & - & 32.686 & - & - & - \\
\hline Lettuce (1eaf) & - & - & - & - & - & - & - & - \\
\hline Me1ons & - & - & - & - & 9,547 & - & - & - \\
\hline $\begin{array}{l}\text { Miscellaneous } \\
\text { vegetables }\end{array}$ & - & - & - & - & - & - & - & - \\
\hline Nectarines & - & - & - & - & - & - & - & - \\
\hline Oats & - & - & - & - & - & - & - & - \\
\hline Onions & - & - & - & - & 26,472 & - & - & - \\
\hline Ornamentals & - & - & - & - & - & - & - & - \\
\hline Other & 85,622 & - & - & - & 76,800 & 16.7 & 1.26 & - \\
\hline Safflower & - & - & - & - & - & - & - & - \\
\hline Sorghum & - & - & - & - & - & - & - & - \\
\hline Squash & - & - & - & - & - & - & - & - \\
\hline Sugar beet & 186 & - & - & - & 32,600 & - & - & - \\
\hline Tomato & - & - & - & - & 1,700 & - & - & - \\
\hline Turf & - & - & - & - & - & - & - & - \\
\hline Turnip & - & - & - & - & - & - & - & - \\
\hline Vector control & - & - & - & - & - & - & - & 0.85 \\
\hline Wheat & - & - & - & - & - & - & - & - \\
\hline Total & 87,455 & 16,669 & 3,622 & 43.6 & 218,197 & 16.7 & 1.26 & 0.85 \\
\hline
\end{tabular}

${ }^{a}$ Data from Ming-Yu Li, Environmental Toxicology Center, University of California, Davis. 
Table 4.12. Pounds of pesticides and herbicides applied in Imperial Valley in 1975. ${ }^{a}$

\begin{tabular}{|c|c|c|c|c|c|c|c|c|}
\hline Uses & $\mathrm{DEF}$ & Demeton & $\begin{array}{l}\text { Diammonium } \\
\text { phosphate }\end{array}$ & Diazinon & $\begin{array}{l}\text { Dicamba, } \\
\text { dimethyl- } \\
\text { arine } \\
\text { silt }\end{array}$ & $\begin{array}{l}\text { Dicanba, } \\
\text { dimathyl- } \\
\text { amine } \\
\text { salt, ather } \\
\text { related }\end{array}$ & $\begin{array}{l}\text { behto- } \\
\text { benil }\end{array}$ & 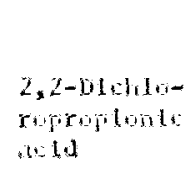 \\
\hline Alfalfa & - & 3.9 & 119.6 & 34,451 & - & - & - & - \\
\hline Asparagus & - & 64.45 & - & - & - & - & - & - \\
\hline Barley & - & - & - & - & - & - & - & $=$ \\
\hline Cabbage & - & - & - & - & - & - & - & - \\
\hline Carrot & - & - & - & 914 & - & - & - & - \\
\hline Cauliflower & - & 2.0 & - & - & - & - & - & - \\
\hline citrus & - & - & 38.56 & 94 & - & - & - & - \\
\hline Corn & - & - & - & 17 & - & - & - & - \\
\hline Cutton & 60,146 & 258 & 290 & - & - & - & - & 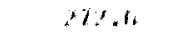 \\
\hline Cucumber & - & - & - & 224 & - & - & - & - \\
\hline $\begin{array}{l}\text { Fal low } \\
\text { farml and }\end{array}$ & - & - & - & - & - & - & - & - \\
\hline $\mathrm{Fin:3}$ & - & - & - & - & - & - & - & - \\
\hline $\begin{array}{l}\text { Forage, hay, } \\
\text { and silage }\end{array}$ & - & - & - & 20 & - & - & - & - \\
\hline Garlic & - & - & 6.48 & - & - & - & - & - \\
\hline $\begin{array}{l}\text { Grassies, } \\
\text { grains, and } \\
\text { liber crops }\end{array}$ & - & - & - & - & - & - & - & - \\
\hline Lettuce (head) & - & 38 & $17 \%$ & $\therefore 2 B$ & - & - & - & - \\
\hline Lettuce (leal) & - & - & - & - & - & - & - & - \\
\hline Molun: & - & - & - & 1,765 & - & - & - & - \\
\hline $\begin{array}{c}\text { Miscellaneous } \\
\text { vegetables }\end{array}$ & - & - & 13.1 & 245 & - & - & - & - \\
\hline Nectarines & - & - & - & - & - & - & - & - \\
\hline Oats & - & - & - & - & - & - & - & - \\
\hline Onions: & - & - & - & 629 & - & - & - & - \\
\hline Ornamentals & - & - & - & 75 & - & - & - & - \\
\hline other & - & - & - & 326 & 34.9 & 3.63 & $27 . x$ & - \\
\hline Safflower & - & - & 12.41 & - & - & - & - & - \\
\hline Sorrhum & - & 135 & - & 112 & 50.9 & 8.21 & - & - \\
\hline Squash & - & - & - & 869 & - & - & - & - \\
\hline Sugar beet & - & - & 245.5 & 1,886 & - & - & - & - \\
\hline Tomato & - & 858 & 32.2 & 1.305 & - & - & - & - \\
\hline Turf & - & - & - & 146 & - & - & - & - \\
\hline Turnip & - & - & - & 28 & 191.6 & 30.9 & - & - \\
\hline Vector control & - & - & - & - & - & - & - & - \\
\hline Wheat & - & 1,241 & 68.7 & - & 5,111 & 983 & - & - \\
\hline Total & 60,146 & 2,600 & 1,004 & 45,945 & 6,389 & 1,030 & 27.5 & 272.6 \\
\hline
\end{tabular}

ada from Ming-Yu l.i, Environmental Toxicology Center, University of Califarnia, Davis. 
Table 4.12. Pounds of pesticides and herbicides applied in Imperial Valley in 1975 a $^{\mathrm{a}}$

\begin{tabular}{|c|c|c|c|c|c|c|c|c|}
\hline Uses & Dieldrin & $\begin{array}{l}\text { Diethyl- } \\
\text { amine } \\
\text { salt of } \\
\text { coconut } \\
\text { fatty acid }\end{array}$ & $\begin{array}{l}\text { Dimetho- } \\
\text { ate }\end{array}$ & $\begin{array}{l}\text { Dipha- } \\
\text { cindne, } \\
\text { sodium } \\
\text { salt }\end{array}$ & $\begin{array}{l}\text { Diphen- } \\
\text { amid }\end{array}$ & $\begin{array}{l}\text { Disodium } \\
\text { octaborate } \\
\text { tetra- } \\
\text { hydrate }\end{array}$ & Di-Syston & Diuron \\
\hline Alfalfa & - & - & 1,839 & - & - & - & 16,722 & - \\
\hline Asparagus & - & - & - & - & - & - & - & 4,974 \\
\hline Barley & - & - & - & - & - & - & 1,342 & - \\
\hline Cabbage & - & - & 21 & - & - & - & - & - \\
\hline Carrot & - & - & - & - & - & - & - & - \\
\hline Cauliflower & - & - & - & - & - & - & - & - \\
\hline Citrus & - & - & $1,786.3$ & - & - & - & - & - \\
\hline Corn & - & - & - & - & - & - & - & - \\
\hline Cotton & 85.9 & - & 454 & - & 11 & - & 7,510 & 29.2 \\
\hline Cucumber & - & - & - & - & - & - & - & - \\
\hline $\begin{array}{l}\text { Fallow } \\
\text { farmland }\end{array}$ & - & - & - & - & - & - & - & - \\
\hline Figs & - & - & - & - & - & - & - & - \\
\hline $\begin{array}{l}\text { Forage, hay, } \\
\text { and silage }\end{array}$ & - & - & - & - & - & - & - & - \\
\hline Garlic & - & - & - & - & - & - & - & - \\
\hline $\begin{array}{l}\text { Grasses, } \\
\text { grains, and } \\
\text { fiber crops }\end{array}$ & - & - & - & - & - & - & - & - \\
\hline Lettuce (head) & - & - & 1,201 & - & - & - & 1,521 & - \\
\hline Lettuce (leaf) & - & - & - & - & - & - & - & - \\
\hline Melons & - & - & $1,395.2$ & - & - & - & - & - \\
\hline $\begin{array}{c}\text { Miscellaneous } \\
\text { vegetables }\end{array}$ & - & - & 22.0 & - & - & - & - & - \\
\hline Nectarines & - & - & - & - & - & - & - & - \\
\hline Oats & - & - & - & - & - & - & 47.8 & - \\
\hline Onions & - & - & - & - & - & - & - & - \\
\hline Ornamentals & - & - & - & - & - & - & - & - \\
\hline Other & - & - & - & 0.01 & 2 & 183 & - & 7,978 \\
\hline Safflower & - & - & 29.3 & - & - & - & - & - \\
\hline Sorghum & - & - & - & - & - & - & 1,495 & - \\
\hline Squash & - & - & - & - & - & - & - & - \\
\hline Sugar beet & - & - & - & - & - & - & 1,787 & - \\
\hline Tomato & - & - & 2,990 & - & 3,561 & - & - & - \\
\hline Turf & - & - & - & - & - & - & - & - \\
\hline Turnip & - & - & - & - & - & - & - & - \\
\hline Vector control & - & - & - & - & - & - & - & - \\
\hline Wheat & - & 0.4 & - & - & - & - & 33,751 & - \\
\hline Total & 85.9 & 0.4 & 9,737 & 0.01 & 3,574 & 183 & 64,176 & 12,981 \\
\hline
\end{tabular}

a Data from Ming-Yu Li, Environmental Toxicology Center, University of California, Davis. 
Table 4.12. Pounds of pesticides and herbicides applied in Imperial Valley in 1975. a

\begin{tabular}{|c|c|c|c|c|c|c|c|c|}
\hline Uses & DNBP & $\begin{array}{l}\text { DNBP, } \\
\text { amine }\end{array}$ & $\begin{array}{l}\text { DNBP } \\
\text { ammonium }\end{array}$ & Dylox & Dypene & Endosulfan & $\begin{array}{l}\text { Endothall, di(n,n- } \\
\text { diethylally lamina) }\end{array}$ & 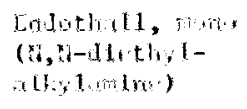 \\
\hline Alfalfa & 11,462 & 707.6 & - & 3,899 & - & 4.0 & - & - \\
\hline Asparagus & - & - & - & - & - & - & - & - \\
\hline Barley & - & - & - & - & - & - & - & - \\
\hline Cabbage & - & - & - & - & - & 98 & - & - \\
\hline Carrot & - & - & - & 476 & - & - & - & - \\
\hline Cauliflower & - & - & - & - & - & 22.5 & - & - \\
\hline Citru: & - & - & - & - & - & - & - & - \\
\hline Corn & - & - & - & - & - & - & - & - \\
\hline Cutton & - & - & - & 238 & - & 3,777 & 107.6 & $\therefore \div 101$ \\
\hline Cucumber: & - & - & - & - & - & - & - & - \\
\hline $\begin{array}{l}\text { Fallow } \\
\text { farmland }\end{array}$ & 518 & - & - & - & - & - & - & - \\
\hline Figa & - & - & - & - & - & - & - & - \\
\hline $\begin{array}{l}\text { Forage, hay, } \\
\text { and silage }\end{array}$ & - & - & - & - & - & - & - & - \\
\hline Garlic & - & - & 170 & - & 700 & - & - & - \\
\hline $\begin{array}{l}\text { Grasses, } \\
\text { grains, and } \\
\text { fiber crops }\end{array}$ & - & - & - & - & - & - & - & $\cdot$ \\
\hline Lettuce (head) & - & - & - & - & - & 73,666 & - & - \\
\hline Lettuce (leaf) & - & - & - & - & - & 62 & - & - \\
\hline Melons & - & - & - & 318.7 & - & 172 & - & - \\
\hline $\begin{array}{c}\text { Miscellaneous } \\
\text { vegetables }\end{array}$ & - & - & - & - & - & 206 & - & - \\
\hline Nectarines & - & - & - & - & - & - & - & - \\
\hline Oats & - & - & - & - & - & - & - & - \\
\hline Onions & - & - & 290 & - & - & - & - & - \\
\hline Ornamentals & - & - & - & - & - & - & - & - \\
\hline other & 2,955 & - & - & - & - & - & - & - \\
\hline Safflower & - & $\cdots$ & - & - & - & - & - & - \\
\hline Sorghum & - & - & - & - & - & - & - & - \\
\hline Squash & - & - & - & - & - & 236 & - & - \\
\hline Sugar bieet & - & - & - & 2,576 & - & 370 & - & - \\
\hline Tomato & - & - & - & - & - & 2,503 & - & - \\
\hline Turf & - & - & - & - & - & - & - & - \\
\hline Iurnip & - & - & - & - & - & 29 & - & - \\
\hline Vecton control & - & - & - & - & - & - & - & - \\
\hline Wheat & - & - & - & - & - & - & - & - \\
\hline Total & 14,935 & 707.6 & 460 & 7,508 & 700 & 81,147 & 107.6 & 2,861 \\
\hline
\end{tabular}

a Data from Ming-Yu Li, Environmental Toxicology Center, University of California, Davis. 
Table 4.12. Pounds of pesticides and herbicides applied in Imperial Valley in $1975 .^{a}$

\begin{tabular}{|c|c|c|c|c|c|c|c|c|}
\hline Uses & Eptam & Ethephon & Ethion & $\begin{array}{l}\text { Ethylene } \\
\text { dibromide }\end{array}$ & $\begin{array}{l}\text { Ethylene } \\
\text { dichloride }\end{array}$ & Flocmeturon & Folex & $\begin{array}{l}\text { Folex, } \\
\text { other } \\
\text { related }\end{array}$ \\
\hline Alfalfa & 959 & - & - & - & - & - & - & - \\
\hline Asparagus & - & - & - & - & - & - & - & - \\
\hline Barley & - & - & - & - & - & - & - & - \\
\hline Cabbage & - & - & - & - & - & - & - & - \\
\hline Carrot & - & - & - & 86,842 & - & - & - & - \\
\hline Cauliflower & - & - & - & - & - & - & - & - \\
\hline Citrus & - & - & 80 & - & - & - & - & - \\
\hline Corn & - & - & - & - & - & - & - & - \\
\hline Cotton & - & - & - & - & - & 20 & 12,534 & 669 \\
\hline Cucumber & - & - & - & - & - & - & - & - \\
\hline $\begin{array}{l}\text { Fallow } \\
\text { farmland }\end{array}$ & - & - & - & - & - & - & - & - \\
\hline Figs & - & - & - & - & - & - & - & - \\
\hline $\begin{array}{l}\text { Forage, hay, } \\
\text { and silage }\end{array}$ & - & - & - & - & - & - & - & - \\
\hline Garlic & - & - & - & - & - & - & - & - \\
\hline $\begin{array}{l}\text { Grasses, } \\
\text { grains, and } \\
\text { fiber crops }\end{array}$ & - & - & - & - & - & - & - & - \\
\hline Lettuce (head) & - & - & - & 46,363 & - & - & - & - \\
\hline Lettuce (leaf) & - & - & - & - & - & - & - & - \\
\hline Melons & - & - & - & 13,200 & - & - & - & - \\
\hline $\begin{array}{c}\text { Miscellaneous } \\
\text { vegetables }\end{array}$ & - & - & - & - & - & - & - & - \\
\hline Nectarines & - & - & - & - & - & - & - & - \\
\hline Oats & - & - & - & - & - & - & - & - \\
\hline Onions & - & - & - & - & - & - & - & - \\
\hline Ornamentals & - & - & - & - & - & - & - & - \\
\hline Other & - & - & - & - & 1,123 & - & - & - \\
\hline Safflower & - & - & - & - & - & - & - & - \\
\hline Sorghum & - & - & - & - & - & - & - & - \\
\hline Squash & - & - & - & - & - & - & - & - \\
\hline Sugar beet & - & - & - & - & - & - & - & - \\
\hline Tomato & - & 21.6 & - & - & - & - & - & - \\
\hline Turf & - & - & - & - & - & - & - & - \\
\hline Turnip & - & - & - & - & - & - & - & - \\
\hline Vector control & - & - & - & - & - & - & - & - \\
\hline Wheat & - & - & - & - & - & - & - & - \\
\hline Total & 959 & 21.6 & 80 & 146,405 & 1,123 & 20 & 12,534 & 669 \\
\hline
\end{tabular}

a Data from Ming-Yu Li, Environmental Toxicology Center, University of California, Davis. 
Table 4.12. Pounds of pesticides and herbicides applied in Imperial Valley in $1975 .^{a}$

\begin{tabular}{|c|c|c|c|c|c|c|c|c|}
\hline Uses & $\begin{array}{l}\text { Formetanate } \\
\text { hydro- } \\
\text { chloride }\end{array}$ & Funda1 & $\begin{array}{l}\text { Fundal } \\
\text { SP }\end{array}$ & $\begin{array}{l}\text { Glyphosate, } \\
\text { isopropyl- } \\
\text { amine salt }\end{array}$ & Gophaside & Guthion & $\begin{array}{l}\text { Hedebloro- } \\
\text { phent: } \\
\text { sodium att }\end{array}$ & $\lim , \operatorname{lin}$ \\
\hline Alfalfa & 5,385 & 59 & - & - & - & - & - & $2,11 \%$ \\
\hline Asparagus & - & - & - & - & - & - & - & - \\
\hline Barley & - & - & - & - & - & - & - & - \\
\hline Cabbage & - & 33 & - & - & - & - & - & - \\
\hline Carrot & - & - & - & - & - & - & - & - \\
\hline Cauliflower & - & - & - & - & - & - & - & - \\
\hline Citrus & 11.4 & - & - & - & - & - & - & - \\
\hline Corn & - & - & - & - & - & - & - & - \\
\hline Cotton & - & 55,602 & 3,041 & - & - & 22,724 & 877 & - \\
\hline Cucumber & - & - & - & - & - & 20 & - & - \\
\hline $\begin{array}{l}\text { Fallow } \\
\text { farmland }\end{array}$ & - & - & - & - & - & - & - & - \\
\hline Figs & - & - & - & - & - & - & - & - \\
\hline $\begin{array}{l}\text { Forage, hay, } \\
\text { and silage }\end{array}$ & - & - & - & - & - & - & - & - \\
\hline Garlic & - & - & - & - & - & - & - & - \\
\hline $\begin{array}{l}\text { Grasses, } \\
\text { grains, and } \\
\text { fiber crops }\end{array}$ & - & - & - & - & - & - & - & - \\
\hline Lattuce (head) & - & - & - & - & - & - & - & - \\
\hline Lettuce (leaf) & - & - & - & - & - & - & - & - \\
\hline Melons & - & - & - & - & - & 70 & - & - \\
\hline $\begin{array}{c}\text { Miscellaneous } \\
\text { vegetables }\end{array}$ & - & - & - & - & - & - & - & - \\
\hline Nectarines & - & - & - & - & - & 4 & - & - \\
\hline oats & - & - & - & - & - & - & - & - \\
\hline Onton: & - & - & - & - & - & 169 & - & - \\
\hline Ornamentials & - & - & - & - & - & - & - & - \\
\hline other & - & - & - & 156 & 4.54 & - & - & - \\
\hline Safflower & - & - & - & - & - & - & - & - \\
\hline Sorghum & - & - & - & - & - & - & - & - \\
\hline Squash & - & - & - & - & - & - & - & - \\
\hline Sugar beet & - & - & - & - & - & - & - & - \\
\hline Tomato & - & - & - & - & - & 631 & - & - \\
\hline Turf & - & - & - & - & - & - & - & - \\
\hline Turnip & - & - & - & - & - & - & - & - \\
\hline Vector control & - & - & - & - & - & - & - & - \\
\hline Wheat & - & - & - & - & - & - & - & - \\
\hline Tatis & 5,396 & 55,694 & 3,041 & 156 & 4.54 & 23,618 & 877 & $2,16 n t$ \\
\hline
\end{tabular}

Data from Ming-Yu Li, Environmental Toxicology Center, University of California, Divi:. 
Table 4.12. Pounds of pesticides and herbicides applied in Imperial Valley in $1975 .^{a}$

\begin{tabular}{|c|c|c|c|c|c|c|c|c|}
\hline Uses & IPC & $\begin{array}{l}\text { 2-Iso- } \\
\text { valeryl- } \\
1-1,3 \text { Inda- } \\
\text { nenedione, } \\
\text { calcium } \\
\text { salt }\end{array}$ & Karathane & Kelthane & Kerb & Leptophos & $\begin{array}{l}\text { Leptophos, } \\
\text { other } \\
\text { related }\end{array}$ & $\begin{array}{l}\text { Lignin, } \\
\text { sulfonic } \\
\text { acid }\end{array}$ \\
\hline$\overline{\text { Alfalfa }}$ & 57,515 & - & - & 318 & - & - & - & - \\
\hline Asparagus & - & - & - & - & - & - & - & - \\
\hline Barley & - & - & - & - & - & - & - & - \\
\hline Cabbage & - & - & - & - & - & - & - & - \\
\hline Carrot & - & - & - & - & - & - & - & - \\
\hline Cauliflower & - & - & - & - & - & - & - & - \\
\hline Citrus & - & - & - & 244.5 & - & - & - & 54.5 \\
\hline Corn & - & - & - & - & - & - & - & - \\
\hline Cotton & - & - & - & 59.8 & - & - & - & - \\
\hline Cucumber & - & - & - & 42 & - & - & - & - \\
\hline $\begin{array}{l}\text { Fallow } \\
\text { farmland }\end{array}$ & - & - & - & - & - & - & - & - \\
\hline Figs & - & - & - & - & - & - & - & - \\
\hline $\begin{array}{l}\text { Forage, hay, } \\
\text { and silage }\end{array}$ & - & - & - & - & - & - & - & - \\
\hline Garlic & - & - & - & - & - & - & ${ }^{-}-$ & - \\
\hline $\begin{array}{l}\text { Grasses, } \\
\text { grains, and } \\
\text { fiber crops }\end{array}$ & - & - & - & - & - & - & - & - \\
\hline Lettuce (head) & 14,492 & - & - & - & 1,588 & - & - & - \\
\hline Lettuce (leaf) & - & - & - & - & - & - & - & - \\
\hline Melons & - & - & 363 & 6,117 & - & - & - & - \\
\hline $\begin{array}{l}\text { Miscellaneous } \\
\text { vegetables }\end{array}$ & - & - & - & - & - & - & - & - \\
\hline Nectarines & - & - & - & $\stackrel{-}{2}+x$ & - & - & - & - \\
\hline Oats & - & - & - & - & - & - & - & - \\
\hline Onions & - & - & - & - & - & - & - & - \\
\hline Ornamentals & - & - & - & - & - & - & - & - \\
\hline Other & - & 0.33 & - & - & - & - & - & - \\
\hline Safflower & - & - & - & - & - & - & - & - \\
\hline Sorghum & - & - & - & - & - & - & - & - \\
\hline Squash & - & - & 96.5 & - & - & - & - & - \\
\hline Sugar beet & 128,251 & - & - & - & - & - & - & - \\
\hline Tomato & - & - & - & 63 & - & 914.5 & 136.6 & - \\
\hline Turf & - & - & - & - & 638 & - & - & - \\
\hline Turnip & - & - & - & - & - & - & - & - \\
\hline Vector control & - & - & - & - & - & - & - & - \\
\hline Wheat & - & - & - & - & - & - & - & - \\
\hline Total & 200,258 & 0.33 & 460 & 6,845 & 2,226 & 914.5 & 136.6 & 54.5 \\
\hline
\end{tabular}

a Data from Ming-Yu Li, Environmental Toxicology Center, University of California, Davis. 

Table 4.12. Pounds of pesticides and herbicides applied in Imperial Valley in

\begin{tabular}{|c|c|c|c|c|c|c|c|c|}
\hline Uses & $\begin{array}{l}\text { Lignin, } \\
\text { sulfonic } \\
\text { acid } \\
\text { (Zn, Mn, } \\
\text { Fe salts) }\end{array}$ & Lindane & Linuron & $\begin{array}{l}\text { Nagnesium } \\
\text { sulfate }\end{array}$ & Balathion & Maneb & $\begin{array}{l}\text { Monch, } \\
\text { other } \\
\text { relinted }\end{array}$ & 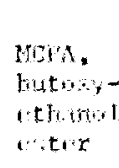 \\
\hline Alfalfa & - & - & - & - & 35,965 & - & - & - \\
\hline Asparagus & - & 8.5 & - & - & 1,173 & - & - & - \\
\hline Barley & - & - & - & - & 320 & - & - & - \\
\hline Cabbage & - & - & - & - & 487 & 76 & - & - \\
\hline Carrot & - & - & 5,398 & - & 171 & - & - & - \\
\hline Caulif lower & - & - & - & - & 143 & - & - & - \\
\hline Citrus & - & - & - & 54.5 & - & - & - & - \\
\hline $\operatorname{Corn}$ & - & - & - & - & - & - & - & - \\
\hline Cotton & - & - & - & - & 761 & - & - & - \\
\hline Cucumber & - & - & - & - & 365 & - & - & - \\
\hline $\begin{array}{l}\text { Fallow } \\
\text { farmland }\end{array}$ & - & - & - & - & - & - & - & - \\
\hline Figs & - & - & - & - & - & - & - & - \\
\hline $\begin{array}{l}\text { Forage, hay, } \\
\text { and silage }\end{array}$ & - & - & - & - & 95.9 & - & - & - \\
\hline Garlic & - & - & - & - & 108 & - & - & - \\
\hline $\begin{array}{l}\text { Grasses, } \\
\text { grains, and } \\
\text { fiber crops }\end{array}$ & - & - & - & - & - & - & - & - \\
\hline Lettuce (head) & - & - & - & - & 36,362 & 3,248 & - & - \\
\hline Lettuce (1eaf) & - & - & - & - & 20 & - & - & - \\
\hline Melons & - & - & - & - & 2,448 & 8,283 & 132 & - \\
\hline $\begin{array}{l}\text { Misce11:aneous } \\
\text { veget:ables }\end{array}$ & - & - & - & - & 577 & - & - & - \\
\hline Nectarines & - & - & - & - & - & - & - & - \\
\hline oats & - & - & - & - & - & - & - & - \\
\hline Onions & - & - & - & - & 4,783 & 4,953 & 1,549 & - \\
\hline ornamentals & - & - & - & - & - & - & - & - \\
\hline other & - & - & 2.0 & - & 75 & - & - & - \\
\hline Safflower & - & - & - & - & - & - & - & - \\
\hline Sorghum & - & - & - & - & - & - & - & - \\
\hline Squash & - & - & - & - & 1,198 & - & - & - \\
\hline Sugar beet & 30.1 & - & - & - & 20,850 & - & - & - \\
\hline Tomato & - & 26.3 & - & - & 542 & - & - & - \\
\hline Turf & - & - & - & - & 93.7 & - & - & - \\
\hline Turnip & - & - & - & - & 153.6 & - & - & - \\
\hline Vector control & - & - & - & - & - & - & - & - \\
\hline Wheat & - & - & - & - & - & - & - & $31 \pi$ \\
\hline Total 1 & 30.1 & 34.8 & 5,400 & 54.5 & 106,234 & 16,560 & 1,681 & 310 \\
\hline
\end{tabular}

a Data from Ming-Yu Li, Environmental Toxicology Center, University wi Califurnia, Davis. 
Table 4.12. Pounds of pesticides and herbicides applied in Imperial Valley in 1975. ${ }^{\mathrm{a}}$

\begin{tabular}{|c|c|c|c|c|c|c|c|c|}
\hline Uses & $\begin{array}{l}\text { MCPA, } \\
\text { dimethyl- } \\
\text { amine } \\
\text { salt }\end{array}$ & $\begin{array}{l}\text { MCPA, } \\
\text { sodium salt }\end{array}$ & Meta-Syston & Methony 1 & $\begin{array}{l}\text { Methyl } \\
\text { bromide }\end{array}$ & $\begin{array}{l}\text { Methyl } \\
\text { parathion }\end{array}$ & Mineral oil & Monitor \\
\hline Alfalfa & 24.5 & - & 176 & 38,454 & - & 8,251 & - & - \\
\hline Asparagus & - & - & - & - & - & - & - & - \\
\hline Barley & - & - & - & - & - & 35.5 & - & - \\
\hline Cabbage & - & - & - & 575 & - & 278 & - & 475 \\
\hline Carrot & - & - & - & 414 & - & 495 & - & - \\
\hline Cauliflower & - & - & - & 147 & - & 118 & - & - \\
\hline Citrus & - & - & - & - & - & - & - & - \\
\hline Corn & - & - & - & 30.8 & - & 6.0 & - & - \\
\hline Cotton & - & - & - & 56,417 & - & 41,041 & - & 1,314 \\
\hline Cucumber & - & - & 105 & 18 & - & - & - & - \\
\hline $\begin{array}{l}\text { Fallow } \\
\text { farmland }\end{array}$ & - & - & - & - & - & - & - & - \\
\hline Figs & - & - & - & - & - & - & - & - \\
\hline $\begin{array}{l}\text { Forage, hay, } \\
\text { and silage }\end{array}$ & - & - & - & - & - & - & - & - \\
\hline Garlic & - & - & - & - & - & - & 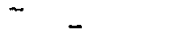 & - \\
\hline $\begin{array}{l}\text { Grasses, } \\
\text { grains, and } \\
\text { fiber crops }\end{array}$ & - & - & - & - & - & - & - & - \\
\hline Lettuce (head) & - & - & 391 & 107,844 & - & 25,653 & - & - \\
\hline Lettuce (leaf) & - & - & - & - & - & 27.9 & - & - \\
\hline Melons & - & - & 1,026 & 695 & - & 69.6 & - & - \\
\hline $\begin{array}{c}\text { Miscellaneous } \\
\text { vegetables }\end{array}$ & - & - & 7.65 & 1,896 & - & 7,552 & - & 1,777 \\
\hline Nectarines & - & - & 4 & - & - & - & - & - \\
\hline Oats & - & - & - & - & - & - & - & - \\
\hline Onions & - & - & - & 82.8 & - & 287.8 & - & - \\
\hline Ornamentals & - & - & - & 452 & - & - & - & - \\
\hline Other & - & - & - & - & 496 & - & - & - \\
\hline Safflower & - & - & - & - & - & - & - & - \\
\hline Sorghum & - & - & 18.3 & 271 & - & 366 & - & - \\
\hline Squash & - & - & 129 & 82.3 & - & - & - & - \\
\hline Sugar beet & - & - & 2,459 & 85,133 & - & 49,398 & - & - \\
\hline Toma to & - & - & - & 18,219 & - & 587 & - & - \\
\hline Turf & - & - & 70 & - & - & - & - & - \\
\hline Turnip & - & - & - & - & - & - & - & - \\
\hline Vector control & - & - & - & - & - & - & 315 & - \\
\hline Wheat & 695 & 680 & - & - & - & 514 & - & - \\
\hline Total & 720 & 680 & 4,386 & 310,731 & 496 & 134,681 & 315 & 3,566 \\
\hline
\end{tabular}

a Data from Ming-Yu Li, Environmental Toxicology Center, University of California, Davis. 
Table 4.12. Pounds of pesticides and herbicides applied in Imperial Valley in $1975 .^{a}$

\begin{tabular}{|c|c|c|c|c|c|c|c|c|}
\hline Uses & MSMA & Naled & $\begin{array}{l}\text { Nonycphenol } \\
\text { polyosy- } \\
\text { ethylene }\end{array}$ & $\begin{array}{l}\text { Parquat } \\
\text { dichloride }\end{array}$ & I arathion & FCNB & Furthint: & $\begin{array}{l}\text { lextens, } \\
\text { ather } \\
\text { ret ited }\end{array}$ \\
\hline$\Lambda l f a l f a$ & - & 95.4 & - & - & 9,300 & - & - & - \\
\hline Asparagus & - & - & - & 829 & - & - & - & - \\
\hline Barley & - & - & - & - & 15 & - & - & - \\
\hline Cabbage & - & - & - & - & 529 & - & 276 & 10.2 \\
\hline Carrot & - & - & - & - & 550 & - & - & - \\
\hline Cauliflower & - & - & - & - & 126 & - & - & - \\
\hline Citrus & - & - & - & - & 96 & - & - & - \\
\hline Corn & - & - & - & - & 12 & - & - & - \\
\hline Cotton & 114 & 417 & - & 1,175 & 60,270 & 7,049 & - & - \\
\hline Cucumber & - & 71.9 & - & - & 6.5 & - & - & - \\
\hline $\begin{array}{l}\text { Fallow } \\
\text { farmland }\end{array}$ & - & - & - & 508 & - & - & - & - \\
\hline Figs & - & - & - & - & - & - & - & - \\
\hline $\begin{array}{l}\text { Forage, hay, } \\
\text { and silage }\end{array}$ & - & - & - & - & - & - & - & - \\
\hline Garlic & - & - & - & - & 56.3 & - & - & - \\
\hline $\begin{array}{l}\text { Grasses, } \\
\text { grain:, and } \\
\text { flber crops }\end{array}$ & - & - & - & - & - & - & - & - \\
\hline Lettuce (head) & - & - & - & - & 31,373 & - & 21,311 & 515 \\
\hline Lettuce (1eaf) & - & 16 & - & - & 23.3 & - & 50 & 4.1 \\
\hline Melons & - & 170 & - & - & 1,054 & - & - & - \\
\hline $\begin{array}{l}\text { Miscellineous } \\
\text { vegetables }\end{array}$ & - & - & - & - & 3,411 & - & - & - \\
\hline Nectarines & - & - & - & - & - & - & - & - \\
\hline outs & - & - & - & - & - & - & - & - \\
\hline Onion: & - & - & - & - & 219 & - & - & - \\
\hline Ornamentals & - & - & - & - & - & - & - & - \\
\hline other & 5,033 & 40 & - & 6.9 & - & - & - & - \\
\hline Safflower & - & - & - & - & - & - & - & - \\
\hline Sorghum & - & - & - & - & 249 & - & - & - \\
\hline Squash & - & 388 & - & - & 337 & - & - & - \\
\hline Sugar beet & - & 120 & - & 30 & 80,165 & - & - & - \\
\hline Tomato & - & 25.5 & - & 51.4 & 1,079 & - & - & - \\
\hline Turf & - & - & - & - & - & - & - & - \\
\hline Turnip & - & - & - & - & - & - & - & - \\
\hline Vector control & - & - & - & - & - & - & - & - \\
\hline Wheat & - & - & 2.76 & - & 530 & - & - & $=$ \\
\hline Total & 5,147 & 1,344 & 2.76 & 2,600 & 189,401 & 7,049 & 21,707 & 31 \\
\hline
\end{tabular}

aData from Ming-Yu Li, Environmental Toxicology Center, Iniversity of California, Davi:. 
Table 4.12. Pounds of pesticides and herbicides applied in Imperial Valley in $1975 .^{a}$

\begin{tabular}{|c|c|c|c|c|c|c|c|c|}
\hline Uses & $\begin{array}{l}\text { Petroleum } \\
\text { distillates }\end{array}$ & $\begin{array}{l}\text { Petroleum } \\
\text { distillates, } \\
\text { aromatic }\end{array}$ & $\begin{array}{l}\text { Petroleum } \\
\text { hydro- } \\
\text { carbons }\end{array}$ & $\begin{array}{l}\text { Petroleum } \\
\text { oil, } \\
\text { unclassi- } \\
\text { fied }\end{array}$ & $\begin{array}{l}\text { Phemme- } \\
\text { diphan }\end{array}$ & Phorate & Phusphalone & Phosdrin \\
\hline Alfalfa & 299 & 137 & 17,379 & 19,867 & - & 1,045 & - & 15,663 \\
\hline Asparagus & 3.4 & - & 198 & - & - & - & - & - \\
\hline Barley & - & 228 & 34.7 & - & - & 1,608 & - & - \\
\hline Cabbage & 1.4 & - & 35.2 & - & - & - & - & 123 \\
\hline Carrot & 13.3 & - & 116 & 179,114 & - & - & - & 351 \\
\hline Cauliflower & - & - & - & - & - & - & - & 21.4 \\
\hline Citrus & - & - & - & - & - & - & 336 & - \\
\hline Corn & - & - & - & - & - & - & - & - \\
\hline Cotton & 47.8 & 167 & 1,338 & 2,627 & - & 1,915 & - & - \\
\hline Cucumber & - & 29.8 & 291 & - & - & - & - & 198 \\
\hline $\begin{array}{l}\text { Fallow } \\
\text { farmland }\end{array}$ & - & - & - & 484 & - & - & - & - \\
\hline Figs & - & - & - & - & - & - & - & - \\
\hline $\begin{array}{l}\text { Forage, hay, } \\
\text { and silage }\end{array}$ & - & - & - & - & - & - & - & - \\
\hline Gar1ic & - & - & 69.2 & - & - & - & - & - \\
\hline $\begin{array}{l}\text { Grasses, } \\
\text { grains, and } \\
\text { fiber crops }\end{array}$ & - & - & - & - & - & - & - & - \\
\hline Lettuce (head) & 4,425 & 359 & 15,547 & 66.2 & - & 13,613 & - & 24,935 \\
\hline Lettuce (leaf) & - & - & 14.6 & - & - & - & - & 196 \\
\hline Melons & 16.2 & 304 & 359 & - & - & - & - & 1,175 \\
\hline $\begin{array}{l}\text { Miscellaneous } \\
\text { vegetables }\end{array}$ & - & - & 135 & 33.2 & - & - & - & 588 \\
\hline Nectarines & - & - & - & - & - & - & - & - \\
\hline Oats & - & 19.8 & - & - & - & - & - & - \\
\hline Onions & - & - & 315 & 33.1 & - & - & - & 1,494 \\
\hline Ornamentals & - & - & - & - & - & - & - & - \\
\hline Other & 4.9 & - & 48,024 & 100 & - & - & - & - \\
\hline Safflower & - & - & - & - & - & - & - & - \\
\hline Sorghum & - & 388 & 39.2 & 50,979 & - & 3,797 & - & 133 \\
\hline Squash & 2.8 & 36.9 & 337 & - & - & - & - & 657 \\
\hline Sugar beet & 24.6 & 24.3 & 119 & - & 2,132 & 39,002 & - & - \\
\hline Tomato & - & 593 & 1,185 & 13.2 & - & - & - & 1,943 \\
\hline Turf & - & 11.2 & - & - & - & 4,159 & - & 281 \\
\hline Turnip & - & - & 38.6 & - & - & - & - & 14.7 \\
\hline Vector control & - & - & - & - & - & - & - & - \\
\hline Wheat & - & 6,174 & - & - & - & 32,169 & - & - \\
\hline Total & 4,838 & 8,472 & 85,575 & 253,316 & 2,132 & 97,308 & 336 & 47,773 \\
\hline
\end{tabular}

Data from Ming-Yu Li, Environmental Toxicology Center, University of California, Davis. 
Table 4.12. Pounds of pesticides and herbicides applied in Imperial Valley in $1975 .^{a}$

\begin{tabular}{|c|c|c|c|c|c|c|c|c|}
\hline Uses & $\begin{array}{l}\text { Phosdrin, } \\
\text { other } \\
\text { related }\end{array}$ & $\begin{array}{l}\text { Phospha- } \\
\text { midon }\end{array}$ & $\begin{array}{l}\text { Phosphami- } \\
\text { don, other } \\
\text { related }\end{array}$ & Phostozid & $\begin{array}{l}\text { Fotassium } \\
\text { pyrophos- } \\
\text { phate }\end{array}$ & Prometone & Frometryn: & Fropazans \\
\hline Alfalfa & 10,458 & - & - & - & - & - & - & - \\
\hline Asparagus & - & - & - & - & - & - & - & - \\
\hline Barley & - & - & - & - & - & - & - & - \\
\hline Cabbage & 82 & - & - & - & - & - & - & - \\
\hline Carrot & 233 & - & - & - & - & - & - & - \\
\hline Cauliflower & $1 / 4.3$ & - & - & - & - & - & - & - \\
\hline Citrus & - & 86 & 4.04 & - & - & - & - & - \\
\hline Corn & - & - & - & - & - & - & - & - \\
\hline Cotton & - & - & - & - & - & 0.48 & 21,674 & 372 \\
\hline Cucumber & 132 & 61.9 & 2.05 & - & - & - & - & - \\
\hline $\begin{array}{l}\text { Fallow } \\
\text { farmland }\end{array}$ & - & - & - & - & - & - & - & - \\
\hline Figs & - & - & - & - & - & - & - & - \\
\hline $\begin{array}{l}\text { Forage, hay, } \\
\text { and silage }\end{array}$ & - & - & - & - & - & - & - & - \\
\hline Garlic & - & - & - & - & - & - & $-\quad-$ & - \\
\hline $\begin{array}{l}\text { Grasses, } \\
\text { grains, and } \\
\text { fiber crops }\end{array}$ & - & - & - & - & - & - & - & - \\
\hline Lettuce (head) & 16,610 & - & - & - & - & - & - & - \\
\hline Lettuce (leaf) & 131 & - & - & - & - & - & - & - \\
\hline Melons: & 782 & - & - & - & - & - & - & - \\
\hline $\begin{array}{c}\text { Miscellaneous } \\
\text { vegetables }\end{array}$ & 393 & - & - & - & - & - & - & - \\
\hline Nectarines & - & - & - & - & - & - & - & - \\
\hline Oats & - & - & - & - & - & - & - & - \\
\hline Onions: & 996 & - & - & - & - & - & - & - \\
\hline Ornamentals & - & - & - & - & - & - & - & - \\
\hline other & - & - & - & 0.55 & - & 1,769 & 117 & - \\
\hline Safflower & - & - & - & - & - & - & - & - \\
\hline Sorghum & 88.5 & - & - & - & - & - & - & $3 \% !$ \\
\hline Squassh & 439 & - & - & - & - & - & - & - \\
\hline Sugar beet & - & - & - & - & - & - & - & - \\
\hline Tomato & 1,297 & $2,24.5$ & 7,43 & - & - & - & - & - \\
\hline Turf & 187 & 3,405 & 112.7 & - & - & - & - & -. \\
\hline Turnip & 9.8 & - & - & - & - & - & - & - \\
\hline Vector control & - & - & - & - & - & - & - & - \\
\hline Wheat & - & - & - & - & 0.84 & - & - & - \\
\hline Total & 31,853 & 3,778 & 126 & 0.55 & 0.84 & 1,770 & $21,0,1$ & 17 \\
\hline
\end{tabular}

"Data from Ming-Yu Li, Environmental Toxtcology Center, University of California, arvi... 
Table 4.12. Pounds of pesticides and herbicides applied in Imperial Valley in $1975 .^{a}$

\begin{tabular}{|c|c|c|c|c|c|c|c|c|}
\hline Uses & Pyrazon & $\begin{array}{l}\text { Pyrazon, } \\
\text { other } \\
\text { related }\end{array}$ & Ro neet & $\begin{array}{l}\text { Ryamodine } \\
\text { alkaloid }\end{array}$ & $\begin{array}{l}\text { Silver } \\
\text { butoxy- } \\
\text { ethanol } \\
\text { ester }\end{array}$ & $\begin{array}{l}\text { Silver } \\
\text { isobutyl } \\
\text { ester }\end{array}$ & Simazine & $\begin{array}{l}\text { Sodtum } \\
\text { cacodylate }\end{array}$ \\
\hline Alfalfa & - & - & - & - & - & - & - & - \\
\hline Asparagus & - & - & - & - & - & - & - & - \\
\hline Barley & - & - & - & - & - & - & 1,496 & - \\
\hline Cabbage & - & - & - & - & - & - & - & - \\
\hline Carrot & - & - & - & - & - & - & - & - \\
\hline Cauliflower & - & - & - & - & - & - & - & - \\
\hline Citrus & - & - & - & 0.98 & - & - & - & - \\
\hline Corn & - & - & - & - & - & - & - & - \\
\hline Cotton & - & - & - & - & - & - & - & 8,153 \\
\hline Cucumber & - & - & - & - & - & - & - & - \\
\hline $\begin{array}{l}\text { Fallow } \\
\text { farmland }\end{array}$ & - & - & - & - & - & - & - & - \\
\hline Figs & - & - & - & - & - & - & - & - \\
\hline $\begin{array}{l}\text { Forage, hay, } \\
\text { and silage }\end{array}$ & - & - & - & - & - & - & - - & - \\
\hline Garlic & - & - & - & - & - & - & - & - \\
\hline $\begin{array}{l}\text { Grasses, } \\
\text { grains, and } \\
\text { fiber crops }\end{array}$ & - & - & - & - & - & - & - & - \\
\hline Lettuce (head) & - & - & - & - & - & - & - & - \\
\hline Lettuce (leaf) & - & - & - & - & - & - & - & - \\
\hline Melons & - & - & - & - & - & - & - & - \\
\hline $\begin{array}{l}\text { Miscellaneous } \\
\text { vegetables }\end{array}$ & - & - & - & - & - & - & - & - \\
\hline Nectarines & - & - & - & - & - & - & - & - \\
\hline Oats & - & - & - & - & - & - & - & - \\
\hline Onions & - & - & - & - & - & - & - & - \\
\hline Ornamentals & - & - & - & - & - & - & - & - \\
\hline Other & - & - & - & - & 4,965 & 34 & 56 & 89.3 \\
\hline Safflower & - & - & - & - & - & - & - & - \\
\hline Sorghum & - & - & - & - & - & - & - & - \\
\hline Squash & - & - & - & - & - & - & - & - \\
\hline Sugar beet & 158 & 25.6 & 21,557 & - & - & - & - & - \\
\hline Tomato & - & - & - & - & - & - & - & - \\
\hline Turf & - & - & - & - & - & - & - & - \\
\hline Turnip & - & - & - & - & - & - & - & - \\
\hline Vector control & - & - & - & - & - & - & - & - \\
\hline Wheat & - & - & - & - & - & - & - & - \\
\hline Total & 158 & 25.6 & 21,557 & 0.98 & 4,965 & 34 & 1,552 & 8,242 \\
\hline
\end{tabular}

a Data from Ming-Yu Li, Environmental Toxicology Center, University of California, Davis. 
Table 4.12. Pounds of pesticides and herbicides applied in Imperial Valley in $1975 .^{a}$

\begin{tabular}{|c|c|c|c|c|c|c|c|c|}
\hline Use: & $\begin{array}{l}\text { Sodium } \\
\text { chlorate }\end{array}$ & $\begin{array}{l}\text { Sodium } \\
\text { tea }\end{array}$ & $\begin{array}{l}\text { Sodium } \\
\text { xylene- } \\
\text { sulfonate }\end{array}$ & Starlicide & Strychnine & Sulfur & $\begin{array}{l}\text { Sulfuret } \\
\text { actel }\end{array}$ & Sagrolds \\
\hline Alfalfa & - & - & - & - & 10 & 480,124 & - & 9,611 \\
\hline A:sparaisua & - & - & - & - & 0.05 & - & - & - \\
\hline Barley & - & - & - & - & 0.04 & - & - & - \\
\hline Cabbage & - & - & - & - & - & - & - & - \\
\hline Carrot & - & - & - & - & 0.47 & - & - & - \\
\hline Caulif lower & - & - & - & - & - & - & - & - \\
\hline Citrus & - & - & - & - & 20.5 & 12,514 & - & - \\
\hline Corn & - & - & - & - & - & - & - & - \\
\hline Cotton & 70,492 & - & - & - & - & $200,0,0$ & - & $\therefore 11$ \\
\hline Cucumber & - & - & - & - & 0.29 & - & - & - \\
\hline $\begin{array}{l}\text { Fullow } \\
\text { farmland }\end{array}$ & - & - & - & - & - & - & - & - \\
\hline Figs & - & - & - & - & 1.31 & - & - & - \\
\hline $\begin{array}{l}\text { Forage, hay, } \\
\text { and silage }\end{array}$ & - & - & - & - & - & - & - & - \\
\hline Garlic & - & - & - & - & - & - & - & - \\
\hline $\begin{array}{l}\text { Grasses, } \\
\text { grains, and } \\
\text { fiber crops }\end{array}$ & - & - & - & - & - & - & - & - \\
\hline Lettuce (head) & - & - & - & - & 186 & - & - & - \\
\hline Lettuce (leaf) & - & - & - & - & 0.98 & - & - & - \\
\hline Melon:3 & - & - & - & - & 2.45 & 203,503 & - & - \\
\hline $\begin{array}{l}\text { Miscellaneous } \\
\text { vegetables }\end{array}$ & - & - & - & - & 0.07 & - & - & - \\
\hline Nectarines & - & - & - & - & - & - & - & - \\
\hline Dats: & - & - & - & - & - & - & - & $\Rightarrow$ \\
\hline Uniuns & - & - & - & - & - & 2,117 & 41,713 & - \\
\hline Ornamentals & - & - & - & - & - & - & - & - \\
\hline other & 62.5 & 27.608 & - & 11.8 & 1.18 & - & - & - \\
\hline saffluwer & - & - & - & - & - & - & $=$ & - \\
\hline Surghum & 7,149 & - & - & - & 0.12 & - & $\rightarrow$ & - \\
\hline Squash & - & - & - & - & 0.22 & $12,6.34$ & - & - \\
\hline Sugar beet & - & - & - & - & 0.07 & $2,455,679$ & - & - \\
\hline Tonato & - & - & - & - & 5.24 & - & - & - \\
\hline T'uri & - & - & - & - & - & 1,225 & - & - \\
\hline Turnip & - & - & - & - & - & - & - & - \\
\hline Vectur control & - & - & - & - & - & - & - & - \\
\hline Wheat & - & - & 0.4 & - & 4.01 & - & - & - \\
\hline Total & 77,704 & 27,608 & 0.4 & 11.8 & 233 & $3,371,073$ & 41,763 & 9.217 \\
\hline
\end{tabular}

Data from Ming-Yu Li, Environmental Toxicology Center, Universi of Californix, Dovis. 
Table 4.12. Pounds of pesticides and herbicides applied in Imperial Valley in $1975 .^{a}$

\begin{tabular}{|c|c|c|c|c|c|c|c|c|}
\hline Uses & Telone & $\begin{array}{l}\text { Terbutryn, } \\
\text { Igran }\end{array}$ & $\begin{array}{l}\text { Terbutryn, } \\
\text { other } \\
\text { related }\end{array}$ & Tok 25 & Toxaphene & Trifluralin & $\begin{array}{l}\text { Vinyl } \\
\text { polymer }\end{array}$ & Warfarin \\
\hline A. falfa & - & - & - & - & - & - & - & - \\
\hline Asparagus & - & - & - & - & - & - & - & - \\
\hline Barley & - & - & - & - & - & - & - & - \\
\hline Cabbage & - & - & - & 192 & - & 30 & - & - \\
\hline Carrot & 46,194 & - & - & - & - & 2,220 & - & - \\
\hline Cauliflower & - & - & - & - & - & 16 & - & - \\
\hline Citrus & - & - & - & - & - & - & - & - \\
\hline Corn & - & - & - & - & - & - & - & - \\
\hline Cotton & - & - & - & - & - & 6,785 & - & - \\
\hline Cucumber & - & - & - & - & - & - & - & - \\
\hline $\begin{array}{l}\text { Fallow } \\
\text { farmland }\end{array}$ & - & - & - & - & - & - & - & - \\
\hline Figs & - & - & - & - & - & - & - & - \\
\hline $\begin{array}{l}\text { Forage, hay, } \\
\text { and silage }\end{array}$ & - & - & - & - & - & - & - & - \\
\hline Garlic & - & - & - & - & - & - & - & - \\
\hline $\begin{array}{l}\text { Grasses, } \\
\text { grains, and } \\
\text { fiber crops }\end{array}$ & - & - & - & - & - & - & - & - \\
\hline Lettuce (head) & 12,480 & - & - & - & 568 & - & - & - \\
\hline Lettuce (leaf) & - & - & - & - & - & - & - & - \\
\hline Melons & 14,118 & - & - & - & - & 265 & - & - \\
\hline $\begin{array}{l}\text { Miscellaneous } \\
\text { vegetables }\end{array}$ & 4,593 & - & - & - & - & 72 & - & - \\
\hline Nectarines & - & - & - & - & - & - & - & - \\
\hline Oats & - & - & - & - & - & - & - & - \\
\hline Onions & 2,212 & - & - & 727 & - & - & - & - \\
\hline Ornamentals & - & - & - & - & - & - & - & - \\
\hline Other & - & 19.5 & 1.03 & - & - & - & 5.54 & 1.23 \\
\hline Safflower & - & - & - & - & - & - & - & - \\
\hline Sorghum & - & $1 / 8.5$ & 7.81 & - & - & - & - & - \\
\hline Squash & - & - & - & - & - & - & - & - \\
\hline Sugar beet & 30,284 & - & - & 44,473 & - & - & - & - \\
\hline Tomato & - & - & - & - & - & 587 & - & - \\
\hline I'urf & - & - & - & - & - & - & - & - \\
\hline Turnip & - & - & - & - & - & - & - & - \\
\hline Vector control & - & - & - & - & - & - & - & - \\
\hline Wheat & - & - & - & - & - & - & - & - \\
\hline Total & 109,881 & 168 & 8.84 & 45,392 & 568 & 9,875 & 5.54 & 1.23 \\
\hline
\end{tabular}

a Data from Ming-Yu Li, Environmental Toxicology Center, University of California, Davis. 
Table 4.12. Pounds of pesticides and herbicides applied in Imperial Valley in 1975.a

\begin{tabular}{|c|c|c|c|c|c|}
\hline Uses & Xylene & $\begin{array}{l}\text { Xylene-range } \\
\text { aromatic } \\
\text { solvents }\end{array}$ & Zinc & $\begin{array}{l}\text { Zinc } \\
\text { sulfate }\end{array}$ & Zineb \\
\hline$\Lambda 1 f a l f a$ & 45,331 & 154 & 70.9 & 139 & - \\
\hline Asparagus & - & - & - & - & - \\
\hline Barley & 29.5 & - & - & 1.31 & - \\
\hline Cabbage & 3.06 & 4.11 & 1.4 & 1.0 & - \\
\hline Carrot & 692 & - & - & 0.77 & - \\
\hline Cauliflower & - & 40.4 & - & - & - \\
\hline Citrus & 70.5 & - & - & 68.4 & - \\
\hline Corn & 13.6 & - & - & - & - \\
\hline Cotton & 2,048 & 199 & 93.1 & 589 & - \\
\hline Cucumber & 162 & - & - & 1.76 & - \\
\hline $\begin{array}{l}\text { Fallow } \\
\quad \text { farm1and }\end{array}$ & - & - & - & 1.66 & - \\
\hline Figs & - & - & - & - & - \\
\hline $\begin{array}{l}\text { Forage, hay, } \\
\text { and silage }\end{array}$ & 15.0 & - & - & - & - \\
\hline Garlic & - & - & - & - & - \\
\hline $\begin{array}{l}\text { Grasses, } \\
\text { grains, and } \\
\text { fiber crops }\end{array}$ & - & - & - & 6.59 & - \\
\hline Lettuce (head) & 14,742 & 93,533 & 10.3 & 1,137 & 186 \\
\hline Lettuce (leaf) & 34.2 & 64.6 & - & - & - \\
\hline Melons & 2,679 & 322 & - & 6.25 & - \\
\hline $\begin{array}{c}\text { Miscellaneous } \\
\text { vegetables }\end{array}$ & 1,780 & 245 & 0.26 & 8.39 & - \\
\hline Nectarines & - & - & - & - & - \\
\hline Oats & $\cdot-$ & - & - & - & - \\
\hline Onions & 450 & 7.71 & - & 24.6 & 195 \\
\hline Ornamentals & 56.3 & - & - & - & - \\
\hline other & 13.8 & - & - & - & - \\
\hline Safflower & - & - & - & - & - \\
\hline Sorghum & 259 & - & 1.3 & 0.88 & - \\
\hline Squash & 394 & 426 & - & 15.2 & - \\
\hline
\end{tabular}


Table 4.12. Pounds of pesticides and herbicides applied in Imperial Valley in 1975. a

\begin{tabular}{|c|c|c|c|c|c|}
\hline Uses & Xylene & $\begin{array}{l}\text { Xylene-range } \\
\text { aromatic } \\
\text { solvents }\end{array}$ & Zinc & $\begin{array}{l}\text { Zinc } \\
\text { sulfate }\end{array}$ & Zineb \\
\hline Sugar beet & 10,895 & 867 & 50.7 & 644 & - \\
\hline Tomato & 871 & 1,082 & 12.8 & 146 & - \\
\hline Turf & 800 & - & 1.04 & 87.8 & - \\
\hline Turnip & 21 & - & - & - & - \\
\hline Vector control & - & - & - & - & - \\
\hline Wheat & 39.2 & - & - & 11.9 & - \\
\hline Total & 81,401 & 96,945 & 242 & 2,892 & 381 \\
\hline
\end{tabular}

ata from Ming-Yu Li, Environmental Toxicology Center, University of California, Davis.

calanoid), chironomid larvae along shore pools, Black Mollies present in inlet streams to sea, and a Texas amphipod Corophium Zouisianum.

\section{Food Web Structure}

The dominant primary production of food is by single cell phytoplankton. The two most common dinoflagellates are Exuviezla compressa and Glenodinium sp.; the most common diatoms are Thatassionema nitzschiodes and Nitzschia Zongissima, with Cyclotella sp., Pleurosigma sp., Nitzschia sigmoides, and Amphora sp. being of secondary importance. Finally there is a green algae of the order ChZorococcales. 38,39 Table 4.17 1ists the species found by
Carpelan ${ }^{38}$ and his estimation of maximum population size. Carpelan estimated the average carbon fixation to be $0.75 \mathrm{~g} / \mathrm{m}^{3} / \mathrm{da}$. This is about 4 times greater than the value of 0.175 $\mathrm{g} / \mathrm{m}^{3} / \mathrm{da}$ reported for Long Island Sound. For the total sea, the rate of carbon fixation would be 175,000 tons/yr, using Carpelan's estimate. Feeding on the phytoplankton are four types of abundant zooplankton: Copepods (Cyclops dimorphus), barnacle larvae (Balanus amphitrite denticulatus), pile worm larvae (Neanthes (or Nereis) succinea), and rotifers, Brachionus plicatilis. Young $^{39}$ found his 1967 collections showed results similar to those of Carpelan's 401954 to 56 collections. 
Table 4.13. Recent history of the most importent herbicides and pesticides used in Imperial County $a, b$

\begin{tabular}{|c|c|c|c|c|c|}
\hline & 1970 & 1971 & $1972^{c}$ & 1974 & 1975 \\
\hline Aldicarb & $-\infty$ & --- & -- & $51,28 \%$ & 40,1624 \\
\hline Aromatic petroleum solvents & $-\infty$ & 64,642 & 154,000 & 269,383 & $162,-694$ \\
\hline Atrazine & 20,040 & 2,809 & 12,600 & 6,845 & $H, n 7$ \\
\hline Balan & 23,917 & 13,739 & 20,000 & 18,487 & 15,201 \\
\hline Bidrine & 51,679 & 350 & 500 & 44,273 & $7,21 \mathrm{r}$ \\
\hline Bromoxynil octanoate & 280 & -- & $-\infty$ & 10,465 & 17,36 \\
\hline Carbary 1 & 83,246 & 35,994 & 60,000 & 69,379 & 9,978 \\
\hline Carbofuran & $-\infty$ & -- & 70 & 23,852 & 10.770 \\
\hline Cryolite & 501,507 & 299,995 & 330,000 & 296,514 & 235,16 \\
\hline 2,4-D, alkanolamine salt & & 33,720 & 34,000 & 20,436 & 59,31 \\
\hline $2,4-D$, dimethylamine salt & $\{64,732\}$ & 10,925 & 57,000 & 16,536 & $14,31,3$ \\
\hline Dacthal & $(49,304)$ & 64,140 & 80,000 & $04,0 \$ 1$ & $90,6 h \%$ \\
\hline Dalapon, sodium salt & 6,623 & 91,953 & 134,000 & 9,530 & 37,45 \\
\hline $4(2,4-\mathrm{DB})$ dimethylamine salt & -- & 15,037 & 9,950 & $11,5,1$ & 16,669 \\
\hline D-D mixture & 135,000 & 290,000 & 126,000 & 192,45 & 218,200 \\
\hline $\mathrm{DEF}$ & 61,841 & 561 & 26,000 & 135,718 & 60,100 \\
\hline Diazinon & 32,417 & $2 \therefore, 418$ & 12,000 & 25,700 & $4,9,1$ \\
\hline Di-Syston & 25,515 & 20,652 & 26,000 & 42,497 & $0,17 \%$ \\
\hline Diuron & 1,169 & 0,115 & 15,800 & 15,05 & 12,080 \\
\hline DNBP & 2,520 & $\therefore, 332$ & 16,000 & 31,310 & $1 \div, 13$ \\
\hline Endosulfan & 40,841 & $3:, 751$ & 69,000 & 34,507 & 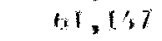 \\
\hline Ethylene dibromide & -- & 598 & 800 & 29.937 & $120,+01$ \\
\hline Folex & 4.838 & 24 & 7,800 & $13, x+0$ & 12,83 \\
\hline Fundal & -- & 66 & 29,000 & 86,254 & 55,69 \\
\hline Guthion & 46,067 & $\operatorname{lij}, 362$ & 24,000 & 47,670 & 29,618 \\
\hline IPC & 123,6014 & 91,205 & 135,000 & 102,127 & 200,257 \\
\hline Malathion & 146,583 & 9,809 & 135,000 & 120,702 & $100, x+2$ \\
\hline Maneb & 46.401 & 1.973 & 12,000 & 7,668 & $16,0,1$ \\
\hline Methony 1 & 142,339 & 45,635 & 160,000 & 285,589 & 310,731 \\
\hline Methyl bromide ${ }^{e}$ & 62,717 & 21,584 & 20,000 & 1,360 & 496 \\
\hline Methyl parathion & 205,947 & 112,129 & 193,000 & 153,163 & $13+, \log 0$ \\
\hline Parathion & 179,154 & 89,408 & 160,000 & 241,690 & 189,400 \\
\hline Perthane & 148.760 & 34.969 & 32.000 & 57.60 & 21,707 \\
\hline Petroleum hydrocarbons & -- & $1,360,000$ & $1,719,000$ & $214,0,07$ & $6 x, 36$ \\
\hline Petroleum oil, unclassified & --- & $x x^{b}$ & 71,000 & 231,900 & 233,11, \\
\hline Phorate & 116,349 & พxA & 170,000 & 80,809 & $97,80 \%$ \\
\hline Phosdrin & 40,009 & 29,819 & 87,000 & 51,005 & 47,772 \\
\hline Phosdrin, other related & -- & 15.438 & 45,000 & 33,960 & $31, \cdots$ \\
\hline Prometryne & 10,899 & 8,908 & 10,400 & 25,834 & 21,401 \\
\hline Ro Neet & 3,817 & 2,128 & 905 & 9,293 & 21,517 \\
\hline Sodium chlorate & 27,797 & 1,871 & 163,000 & 98,300 & 77,704 \\
\hline Sodium tea & -- & 33,069 & 47,000 & 5,964 & 27,600 \\
\hline Sulfur & $1,450,636$ & 549,905 & $1,231,000$ & 449,595 & $1,371,075$ \\
\hline Sulfuric acid & -- & 72,304 & 9,000 & 83,328 & 41,761 \\
\hline Telone & 85,789 & 246,232 & 200,000 & 63,640 & $10 \%, 861$ \\
\hline Tok 25 & 21,795 & $: 2,963$ & 32,000 & 26,205 & 43,392 \\
\hline xylene & $-\infty$ & 26,910 & 68,000 & 53,630 & 31,401 \\
\hline Xylene-range aromatic solvent & -- & $\because 9,493$ & 46,000 & $\therefore 2,072$ & $96,9+5$ \\
\hline
\end{tabular}

Data courtesy of Ming-Yu Li, Environmental Toxicology Center, University ol Calliwrill, Datvis, Califurnia.

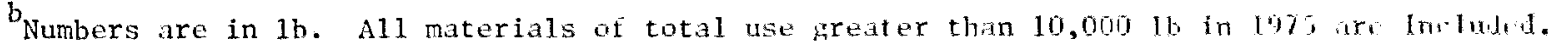

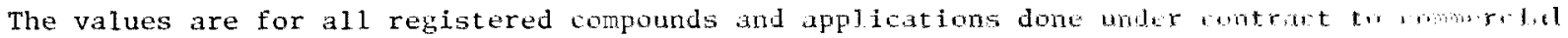
pesticide firms.

CData was poor for 1972; these are estimates. Data for 1973 wiere umatilable.

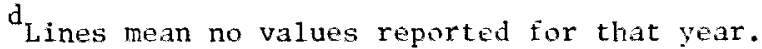

"Included because of historical importance.

$\mathrm{f}_{\mathrm{NA}}=$ not available (missing data). 
Table 4.14. Chemical constitution of Salton Sea water.

\begin{tabular}{lccccccccc}
\hline Year & $\mathrm{Ca}$ & $\mathrm{Mg}$ & $\mathrm{Na}$ & $\mathrm{K}$ & $\mathrm{CO}_{3}$ & $\mathrm{HCO}_{3}$ & $\mathrm{SO}_{4}$ & $\mathrm{C} 1$ & $\%$ TDS \\
\hline $1907^{\mathrm{a}}$ & 2.7 & 1.8 & 30.4 & 0.63 & $1.8^{\mathrm{b}}$ & -- & 13.0 & 46.5 & 3.65 \\
$1910^{\mathrm{a}}$ & 2.3 & 1.6 & 30.8 & 0.58 & 1.1 & -- & 12.6 & 46.5 & 6.04 \\
$1913^{\mathrm{a}}$ & 2.0 & 1.6 & 32.2 & 0.35 & 1.1 & -- & 12.4 & 47.3 & 10.0 \\
$1916^{\mathrm{a}}$ & 1.8 & 1.7 & 32.1 & 0.35 & 0.69 & -- & 12.6 & 47.8 & 16.47 \\
$1929^{\mathrm{a}}$ & 1.4 & 4.1 & $27.2^{\mathrm{C}}$ & -- & 0.71 & -- & 9.5 & 44.3 & 14.43 \\
$1945^{\mathrm{a}}$ & 2.3 & 2.5 & 30.4 & -- & 0.55 & -- & 18.5 & 43.5 & 37.37 \\
$1948^{\mathrm{a}}$ & 2.0 & 2.5 & 29.2 & 0.47 & 0.05 & 0.42 & 18.7 & 42.0 & 40.43 \\
$1955^{\mathrm{a}}$ & 2.3 & 2.8 & 29.5 & 0.67 & 0.06 & 0.47 & 20.2 & 42.8 & 33.68 \\
$1967^{\mathrm{d}}$ & 2.6 & 3.1 & 28.6 & 0.50 & 0.008 & 0.51 & 22.6 & 39.5 & 33.38 \\
$1972^{\mathrm{e}}$ & 2.3 & 2.9 & 27.5 & 0.54 & 0 & 0.51 & 24.5 & 39.4 & 40.2 \\
$1974^{\mathrm{e}}$ & 2.5 & 2.9 & 27.9 & 0.60 & 0.10 & 0.33 & 22.9 & 39.4 & 39.0 \\
$0 \mathrm{cean}$ & 1.2 & 3.7 & 30.6 & 1.1 & -- & 0.04 & 7.7 & 55.5 & 34.5 \\
\hline
\end{tabular}

Data from Carpelan. ${ }^{33}$

${ }^{\mathrm{b}} \mathrm{CO}_{3}+\mathrm{HCO}_{3}$.

$\mathrm{C}_{\mathrm{Na}}+\mathrm{K}$.

${ }^{d}$ Data from California Department of Water Resources. ${ }^{34}$

Data from Spenser. ${ }^{35}$

Table 4.15. Salt constituents of inflow and outflow of Imperial Valley
Irrigation System.

Ion

Inflow, \%

Outf1ow, \%

\begin{tabular}{lcc}
$\mathrm{SO}_{4}$ & 41 & 32 \\
$\mathrm{Na}+\mathrm{K}$ & 17 & 22 \\
$\mathrm{Cl}$ & 16 & 30 \\
$\mathrm{Ca}$ & 12 & 8 \\
$\mathrm{HCO}_{3}$ & 11 & 5 \\
$\mathrm{Mg}$ & 4 & 4 \\
\hline
\end{tabular}


Table 4.16. Fish and invertebrates of the Salton Sea and their origin. 32

\begin{tabular}{|c|c|c|c|c|c|}
\hline Common name & Scientific name & $\begin{array}{l}\text { Introduced } \\
\text { directly }\end{array}$ & Date & $\begin{array}{l}\text { Introduced } \\
\text { through } \\
\text { irrigation } \\
\text { canals }\end{array}$ & $\begin{array}{l}\text { Native } \\
\text { Eisunil }\end{array}$ \\
\hline \multicolumn{6}{|l|}{ Fishes } \\
\hline Orangemouth corvina & Cynoscion xanthulus & $\mathrm{x}$ & $1950-56$ & & \\
\hline Sargo & Anisotremus davidsoni & $\mathrm{X}$ & 1951 & & \\
\hline Gulf croaker & Bairdiella icistius & $\mathrm{x}$ & $1950-51$ & & \\
\hline Mudsucker & Gillichthys mirabilis & $\mathrm{x}$ & 1930 & & \\
\hline Striped mullet & Mugil cephalus & & & $x$ & \\
\hline Threadfin shad & Dorosoma petenense & & & $x^{a}$ & \\
\hline Mosquitofish & Gambusia affinis & & & & $\mathrm{x}^{\mathrm{b}}$ \\
\hline Desert pupfish & Cyprinodon macularius & & & & $X$ \\
\hline \multicolumn{6}{|l|}{ Invertebrates } \\
\hline Pile worm & Neanthes succinea & $\mathrm{x}$ & 1930 & & \\
\hline Amphipod & $\frac{\text { Carinogammarus }}{\text { mucronatum }}$ & $\mathrm{x}$ & 1957 & & \\
\hline Barnacle & Balanus amphitrite & $\mathrm{x}$ & 1944 & & \\
\hline Copepod & Cyclops dimorphus & & & & $\mathrm{x}$ \\
\hline Nematod & Spilophorella sp. & & & & $\mathrm{X}$ \\
\hline Rotifer & Brachionus plicatilis & & & & $X$ \\
\hline Protozoa & Numerous species & & & & $\mathrm{X}$ \\
\hline
\end{tabular}

\footnotetext{
antroduced by man into Colorado River.

${ }^{b}$ Introduced by man into area prior to sea formation.
}

On the bottom of the sea, there is a large detrital pool caused by the tremendous productivity noted above. This detrital pool and productivity is so high that the sea below a depth of $25 \mathrm{ft}$, becomes anoxic during the summer months. 41 The pile worm, Neanthes (or Nereis) succinea, lives on the bottom of the sea, feeding on the detritus. Carpelan and Linsley ${ }^{41}$ estimated the average standing crops of Neanthes to be
$300 \mathrm{lb} /$ acre in the spring, $75 \mathrm{Ib} /$ acre in the summer, and $125 \mathrm{lb} /$ acre in the fall. The low summer value reflects the anoxic condition in the deep piarts of the lake.

The barnacle Balanus amphitrite probably feeds mainly on phytoplankton and po:ssibly on zooplankton. The mullet lugiz cephalus feeds almost exclusively on phytoplant:ton while taking in some zooplankton (copepids). 39,42 The mudsucker 
Table 4.17. Volume of phytoplankton organisms taken from the Salton Sea. 38

\begin{tabular}{lccc}
\hline \multicolumn{1}{c}{ Species } & $\begin{array}{c}\text { Maximum } \\
\mu^{3}\end{array}$ & $\begin{array}{c}\text { Volume of } \\
\text { in } \begin{array}{c}\text { Number/cm } \\
\text { collections }\end{array}\end{array}$ & $\begin{array}{c}\text { maximum number, } \\
\mu^{3} \times 10\end{array}$ \\
\hline Pleurosigma sp. & 800 & 375 & 0.3 \\
Thallasionema nitzschoides & 175 & 9,000 & 1.58 \\
Nitzschia longissima & 200 & 56,000 & 11.2 \\
Cyclotella caspia & 400 & 53,000 & 21.2 \\
Glenodinium sp. & 3,000 & 41,000 & 123. \\
Exuviella compressa & 400 & 63,000 & 25. \\
Westella sp. & 5 & 160,000 & 0.8 \\
Dictyocha sp. & 7,000 & 450 & 3.1 \\
Eutreptia lanorvii & 4,000 & 1,300 & 5.2 \\
\hline
\end{tabular}

Gizlichthys mirabizis eats mainly punky fly larvae as a young fry $(<15 \mathrm{~mm})$, but as an adult its diet is almost entirely Neanthes with occasional shore insects. 43 At certain times of the year, Neanthes are an important item in corvina diets. The thread fin shad Dorosoma petenense eats zooplankton almost exclusively, with small amounts of phytoplankton. 44 The sargo

Anisotremus davidsoni eats primarily pileworms and, secondarily, barnacles. 39,45 During the summer months when Neanthes die from anoxia, Bairdielza often die as we11. 46 Corvina young, Cynoscion xanthulus, feed on copepods, barnacle larvae, and Neanthes. 39,47 After attaining a size of 70 to
$80 \mathrm{~mm}$, corvina switch over exclusively to fish. They feed on Bairdiezza, shad and mudsucker. 39,47 The corvina population in 1956 was estimated to be 40,000 , and in $1957,800,000$.

This food web information is summarized in Fig. 4.6.

\section{$\underline{\text { Abnormalities }}$}

Several implications can be drawn Erom observations made by Hendricks 42 and Whitney 46,47 on abnormalities in Salton Sea fish species. First, abnormalities occur at a high rate in the Salton Sea. Because of a large food supply for all species, abnormal fish can survive well. The exception to this rule is in circumstances of strong competition. In this case, 


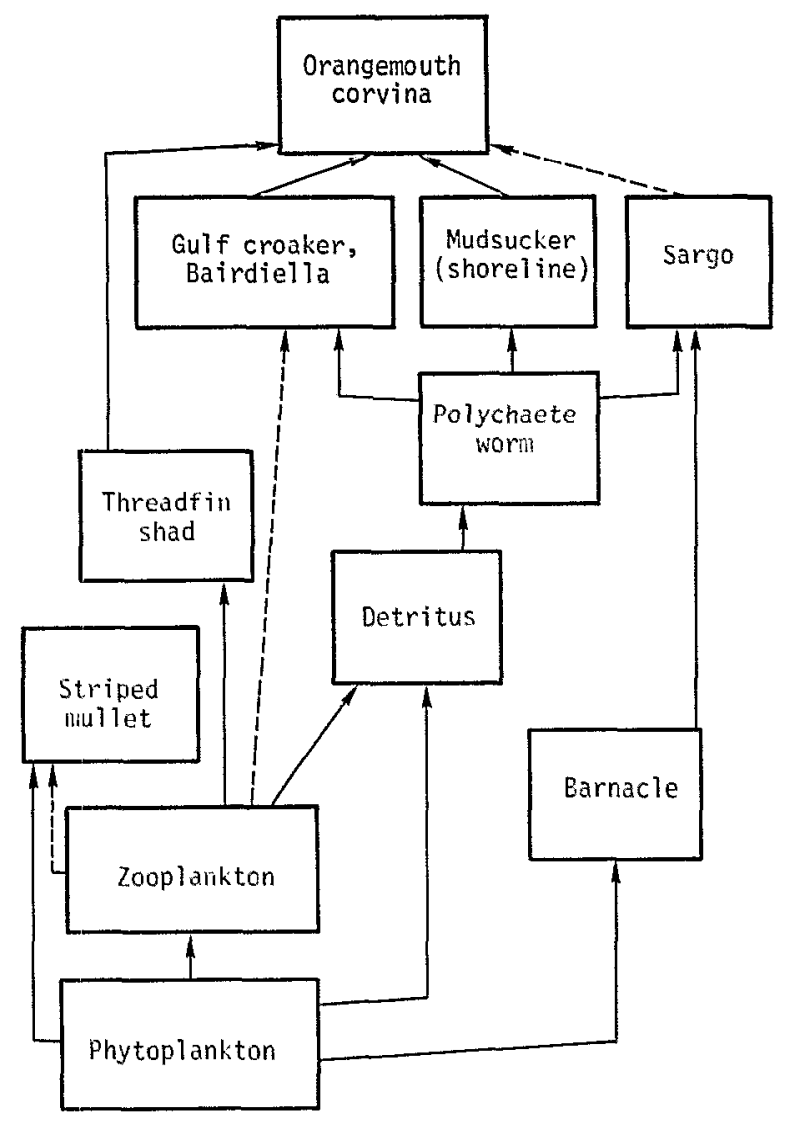

Fig. 4.6. Trophic levels and food web for Salton Sea biota. The major taxa in the Salton Sea food web are shown in their trophic relationships. Solid lines refer to principal diet items; dotted lines refer to occasional diet items.

abnormal fish are weeded out. Specifically, Whitney ${ }^{46}$ notes large numbers of abnormalities in Baixaielza born in 1952. The fish born in 1953 had a far lower incidence of abnormalities and the abnormality rate of the 1952 group dropped in 1953. Whitney attributes this to increased competition in 1953.

\subsection{ENDANGERED SPECIES AND HILDLIFE REFUGES}

Although the Endangered Specie: Conservation Act of 1969 does not set forth specific criteria for determining which species are tinreatened with extinction, it does direct tho Secretary of the Interior to sed: the counsel of specialists and agencies with expertise on the subject, and to rely upon their combined judgement. 48 A later reinforced version of the 1969 Act, the Federal Endangered Species Act of 1973, extends federal authority to the Secretary of the Interior over both migratory, resident, and foreign species of plants and antmals declared endingerud or threatened.

The term endangered species has: been redefined and threatened species has $r \in p l a c e d$ the federal designation of rare.

- Endangered species means any species that is in danger of extinction throughout all or a significant portion of its range, other than a species of class Insecta, determined by the Secretary to constitute a pest whose protection under the provisions of the Act would 
present an overwhelming and overriding risk to man.

- Threatened species means any species that is likely to become an endangered species within the foreseeable future throughout all or a significant portion of its range. 49

The Secretary of the Interior and the California Fish and Game Commission have listed five endangered species known to occur in the Salton Sea KGRA; they are the California brown pelican, southern bald eagle, American peregrine falcon, Yuma clapper rail, and the California least tern. Of these, the Yuma clapper rail is the only nesting inhabitant. The others are largely migratory with sightings of the California least tern reported as casual or accidental. 50

\section{Wildilfe Refuges}

The Salton Sea KGRA region plays a vital role in providing winter quarters for migratory birds, waterfowl, and other water associated birds for a number of reasons. It is located directly on the Pacific Flyway, the Salton Sea National Wildlife Refuge (Fig. 4. $7^{51}$ ) and the Imperial Wildlife Management Area are in the vicinity, and extensive marsh cover and mudflats are within the boundaries of the KGRA.
The Salton Sea National Wildlife Refuge was established in 1930 by President Hoover. This refuge is maintained by the U.S. Bureau of Fisheries and Wildlife as a waterfowl resting and feeding area, as provided in treaties with Mexico and Great Britain (for Canada), and to alleviate agricultural crop depredations by waterfowl in Imperial Valley. ${ }^{52}$ No other comparable area in the West has the tremendous flocks of shorebirds that use the sea and surrounding area every year. Thirty-five species of shorebirds and 47 species of waterbirds, other than swans, ducks, geese, cranes, and rails, have been recorded. 50

The California Department of Fish and Game's Imperial Wildlife Management Area consists of 8,400 acres and is farmed for wildlife food production. The Wister Unit comprises 3,900 acres of the refuge and serves 3 basic purposes:

- To help preserve California's waterfowl resource and associated wildlife,

- To attract and hold wintering waterfowl off Imperial Valley's multimillion dollar agricultural crops, and

- To provide public hunting, fishing, nature study, and related uses. ${ }^{3}$ 


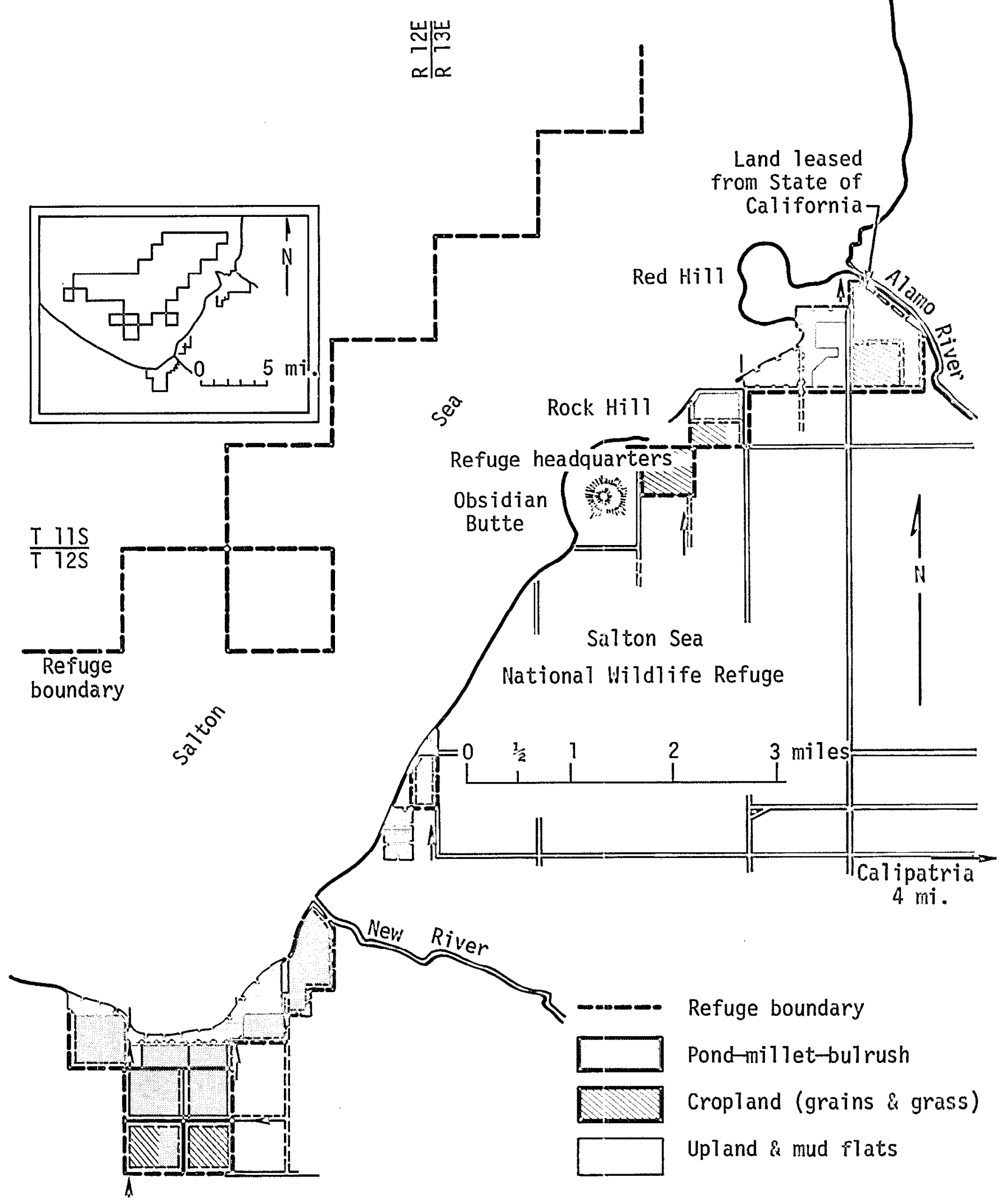

Fig. 4.7. Map of Salton Sea National Vildlife Refuge. Shom are segments of refuge as of June 1971. The refuge is located along southeast shore of the sea. Map taken from U.S. Department of Interior. 51 
The Salton Sea Refuge is also seasonally opened to hunting according to regulations as prescribed by law. McCaskie 53 provides a more detailed accounting of specific shorebird and waterbird use of the Salton Sea.

\section{Endangered Species}

Except as noted the following accounts are taken from Refs. 48 and 49 and describe the five endangered species.

- California brown pelican (Pelecanus occidentalis Califomicus) Description: This is a large, dark gray-brown coastal bird with a bare skin pouch on the underside of its long bill. It flies with its neck and head folded back on its shoulders and with alternate wing flapping and sailing. Adults have mostly white heads and necks, whereas immature birds are dark-headed.

Distribution: This bird breeds locally on islands along the Pacific Coast from Canada to Mexico. It nests on California's Channel Islands, on the coastal islands off lower California, and in the Gulf of California. Post breeding movement of birds progresses northward along the Pacific Coast in late summer and fall.
Status: A 1972 survey indicated that the total population is approximately 100,000 birds with 20,000 pelicans frequenting California's Coast from August through November. California's only remaining nesting colony on Anacapa Island (Ventura County, California), current1y numbering 400 pairs, is incapable of maintaining itself. The decline of the pelican is attributed to poor reproductive success resulting from collapsed eggs because of thin she11s. This is suspected to result from food contamination with DDE (an insecticide) and/or other pollutants.

- Southern bald eagle (HaZiacetus Leucocephatus Leucocephatus) Description: The mature bird of the species is a large, hawk-like soaring bird with mainly dark brown plumage and a pure white head and tail. Immature birds are brown, blotched with white a.11 over.

Distribution: This is the only eagle restricted to North America. It occurs statewide, particularly along the coast and in interior California near large lakes, reservoirs, and wetlands. It nests in the vicinity of large lakes, rivers, 
and reservoirs from Fresno

County north.

Status: The total number of southern bald eagles has diminished rapidly. Reasons for its decline include irresponsible shooting, increase in human population in primary nesting and feeding areas, removal of nest trees, power line electrocution, environmental pollution, and possible reduced reproduction resulting from pesticidecontaminated food ingested by adults.

- American peregrine falcon (Falco peregrinus anatum) Description: This bird is commonly called the duck hawk. It is a mediumsized, blue-gray hawk with long pointed wings and is distinguished from other falcons by its black cap and black cheek patches.

Distribution: The American peregxine, extinct as a breeding bird east of the Rocky Mountains, breeds in California along the coast and in higher inland mountains.

Status: Since the mortality exceeds recruitment, the situation is quite serious. Food chain contamination by persistent pesticides and other contaminants, illegal poaching by falconers, human disturbance, and occasional shooting are contributing to its decline. In the $1940^{\prime} \mathrm{s}$, the breeding bird population in California was 100 pairs. By 1970 this population had declined to $10 \mathrm{birds}$, of which 2 pairs produced 4 young. In 1975, encouraging1y, 8 pairs wero found, 6 of which fledged 14 young.

- California least tern (Stew) albifions biowit) Description: Smallest of the terns, this 9-in-long bird is recognized by its rhite body, gray ringe, black wing tips, blacl-capped head, and black-tipped yellow bi11. Its quick wing beats and hovering action help distinguish it from the larger terns.

Distribution: Migratory. From April to Septomber it apfears along the Pacifle Coast From San Francisco Bay to Central Baja California; breseding colonies are distributed discontinuousiy alon: the coust. The bird's vintering: area is not lino:n although it may inelude coastal arcas of Central or South Americi.

Status: The least tern is threatened with extinction because of continuing destruction of its fey remaining feeding and 
nesting habitats, human disturbance, and animal predators. Nesting colonies require flat areas characterized by complete or nearly complete lack of vegetation, loose substrate, freedom from disturbance, and nearness to an estuary with a good supply of small fish.

Yuma clapper rail (RaZlus Zongirostris yumanensis)

Description: In size and appearance the Yuma clapper rail is similar to a small hen, but has a long, slender, slightly decurved bill, and longish legs. This is the smallest of the clapper rails. It is the only one inhabiting freshwater marshes in southeastern California from April to November.

Distribution: This bird is restricted in the breeding season to cattail-tule marshes along the lower Colorado River from the Colorado Delta in Mexico north to Needles, California, in two small marshes along the lower Gila River, in two small marshes immediately south of Phoenix, Arizona, and at the lower end of the Salton Sea. The species is concentrated mainly in Havasu Lake, Cibola, and Imperial
National Wildlife Refuges. These birds migrate, but their winter range is unknown. In the Imperial Valley, the major habitats are the deltas of the New and Alamo River and the marshlands scattered between the two deltas. These 1ie inside the Salton Sea KGRA.

Status: Of the five endangered species listed, the Yuma clapper rail is of prime interest because one of its limited breeding habitats is located in the Salton Sea area. Members of this species occur in small numbers, are very secretive by nature, and inhabit dense marsh habitats where they are seldom seen. At the Salton Sea site, increasing salinity of the water and destruction of vegetation in Colorado River drainage canals has drastically reduced suitable habitats. From observations made in 1969, it appeared that Yuma clapper rails at the Salton Sea appeared to be confined to the extreme southern end, where cattail-tule marshes were not limited by a high salt content of the water. ${ }^{5}$ The winter habitat of the Yuma clapper rail is unknown; most sources believe the species to be migratory. Neither direct observations nor taped clapper 
rail calls yielded any responses or sightings of Yuma clapper rails along the Colorado River between October and May 1969-70 and 1970-71; clappers further south along the coast of Sonora, Mexico, responded to the same taped calls during all winter months. 53 It was therefore concluded that Yuma clapper rails probably were not present north of the border during the winter.

\subsection{VEGETATIVE COMMUNITIES AND WILDLIFE}

The Salton Sea, Glamis, Dunes, and East Mesa KGRAs have nonagricultural vegetative communities that provide habitats for various types of wildlife. Descriptions of those communities and their related wildlife are summarized from the Department of the Interior Final Environmental Statement for Geothermal Leasing. 54

\section{Salton Sea KGRA}

Vegetation along the shoreline grows for the most part near freshwater drainages. Vegetation consists mainly of salt brush, salt grass, cattails, nutgrass, salt cedar, cane, arrowweed, and heliotrope. Freshwater marshes exist on the State and Federal Wildlife Refuges with alkali bulrush and cattails.
The deltas of the New and Alamo Rivers have populations of coyote, desert fox, raccoon, bobcat, skunl: badger, muskrat, cottontail, jackrabbit, ground squirrel, valley pocket gopher, desert pocket mouse, and desert kangaroo rat.

Numerous shorebirds and waterfowl inhabit the area as discussed earlier. Other desert species such as roatdrunner, cactus wren, and verdin are found in the areas. Amphibians present are southwestern wood home toad, redspotted toad, and bullfrog. Reptiles include zebratailed lizard, fringe-toed lizards, rattlesnakes, western blind snals, and desert glassy snake.

\section{Glamis KGRA}

Thirty percent of the Glamis KGRA is occupied by the bajada (alluvial fans) mized community, consisting of ironwood, creosote, ocotillo, mesquite, paloverde, smol:c tree, desert willow, desert gold, browneyed primrose, dune primrose, and plantago. The forb season is at its height in the late vinter. Tho soil is stable in this community. Approximately $55 \%$ of the Glamia KGRA is in shifting dunes communtties. Thera are several plant species in the dunes communities that are not found elsewhere in the United States. Herbaceous species such as wild sunflower, croton palafoxia, desert 
lily, desert buckwheat are present, as well as sonoran desert trees such as ironwood, desert willow, smoke tree and mesquite.

Fifteen percent of the Glamis KGRA is a creosote forest along the western edge of the KGRA associated with the Coachella Canal. Creosote bush in this area is unusual in nature in both abundance (700 plants/ acre) and size (up to 15 feet). The desert shrub does not go into wilt in this area. This is exceptional for this desert shrub. Ephedra, mesquite, happlopapas, and desert buckwheat are also present and thriving.

These three habitats are the most varied of those in the desert KGRAs. Mammals are similar to East Mesa mammals, but the abundance is greater in the Glamis KGRA. So far, 23 species of mammals, 39 species of birds, 26 species of reptiles, 2 species of amphibians, and 59 species of insects have been identified in the Glamis KGRA. The creosote forest offers a special habitat for desert species. Seeps exist in the forest that contain ponds with complete food chains, including bass as the top carnivore.

\section{Dunes KGRA}

Fifty percent of the Dunes KGRA is covered by a creosote community. Densities of creosote shrubs do not exceed 100 plants/acre and existing plants are under high water stress and are often wilted. Fifty percent of the Dunes KGRA is occupied by dunes communities of the type discussed above. These dunes communities are undistinguished.

\section{East Mesa KGRA}

Fifty percent of the East Mesa KGRA is covered by creosote community. Ninety percent of the shrub species are creosote with the remaining $10 \%$ including ephedra, cheese bush, brittle bush, and coldera. Winter ephemerals are spectacle-pod, desert gold, plantage, and crytantha.

Summer ephemerals include a small number of species such as sand mat, gramma, and mustards. Both summer and winter herbaceous vegetation have a long residence time as litter. Forty percent of the East Mesa KGRA is dunes community similar to those discussed above.

The habitats of the East Mesa KGRA are uniform, and there is not a wide variety of animals present. The species present are abundant, however. The majority of mammals consist of ground squirrels, mice, kangaroo rats, coyote, badger, and bobcat. There are 20 species of reptiles present. Many of these are considered threatened. The desert iguana and western chuckwalla seem to be increasing in the East Mesa 
KGRA. Thirty species of birds have been identified, many of them being winter migrants. Over 50 species of invertebrates have been found, and it is thought that over half are endemic to eastern Imperial County.

\section{REFERENCES}

1. C.M. Finne1, Imperial County Agriculture, 1974. Office of the Agricultura1 Commissioner, E1 Centro, California (1974).

2. C.W. Johnson and V.B. Coleman, "Semi-automatic crop inventory from sequential ERTS-1 imagery," in Symposium on Significant Results obtained from the Earth Resources Technoloyy satellite-1. Vol. I. NASA SP-327. S.C. Freden, E.P. Mercanti, and M.A. Becker, Eds. (NASA Scientific and Technical Information Office, Washington, D.C., 1973), p. 19 .

3. R.R. Thaman and L.W. Senger, "Analysis of Agricultural Resources in the Imperial Valley, California," in Monitoning Earth Resources from Aireraft and Spacecraft, R.N. Colwel1, Ed. (NASA, Washington, D.C., 1971), p. 65 .

4. E.R. Perrier, A.J. Mackenzie, and R.P. Zimmerman, Fhysieal and Chemieal. Properties of Major Imperial ValZey Soils, Agricultural Research Service, Western Region. U.S. Dept. of Agriculture ARSH-17 (1974).

5. Imperial Irrigation District, The Colorado River and Impemial Valtay Soizs, Community Special Services, Imperial Irrigation District, Bulletin No. 373 (1973).

6. D.A. Twogood, Ammal Inventory of Areas Rveeiving baten Yeame 1975, 1974, 1973, Community and Special Services, Imperial Irrigation District, E1 Centro, California (1976).

7. Imperial Irrigation District, Annul Inventory of Areas Receiving Watex (Years 1960 thru 1975), Imperial Irrigation District, E1 Centro, California (1960-75).

8. K.S. Mayberry, Vegetable Production CaZendar Imperial County, University of California Agricultural Extension Service, Imperial County, El Centro, Circular 102 (1974).

9. D.W. Cudney, Imperial County Field Crops CaZendar, University of California Agricultural Extension Service, Imperial County, El Centro, Circular 113 (1967). 
10. C.M. Finne1, Agricultural Commissioner, Imperial County, private communication (1976).

11. L.C. Oglesby and W.O. Wirtz II, The Salton Sea: A tour of the geology and biology of an accidental desert lake, Ives' Community Office, Claremont, California (1973).

12. A.J. Strahorn, E.B. Watson, A.E. Kocher, E.C. Eckmann, and J.B. Hammon, Soil Survey of the EI Centro Area, California. U.S. Department of Agriculture, Bureau of Soils, Gov't. Printing Office, Washington, D.C. (1922).

13. M.T. Kaddah, Imperial Valley Conservation Research Center, Brawley, Ca., private communication (1976).

14. D.E. Pierson, "Imperial County Department of Public Works Presentation to Geothermal Resources Board," in: Compendium of Papers Imperial Valley-Salton Sea Area Geothermal Hearing, State of California Geothermal Resources Board, Section C, (1970).

15. H.O. Buckman and N.C. Brady, The Nature and Properties of Soits (MacMillian Company, New York, 196 ) 6th ed.

16. Imperial Irrigation District, Report for General SoiZ Map, Imperial County, California, Imperial, Calif. (1967).

17. L.B. Grass and W. Simpson, Imperial County Monthly Farm Bureau. Dec. 1968-Jan. 1969.

18. L.B. Grass, J. Soil Water Conserv. 24 (4) 135 (1969).

19. L.B. Grass and A.J. MacKenzie, SuZphur Inst. J., (Spring 1970), p. 8.

20. G.F. Snow, "An overview of Salton Sea Agriculture," in Compendium of Papers Imperial Valley-Salton Sea Geothermal Hearing, Section F, State of California Geothermal Resources Board (1970).

21. K.S. Mayberry, Salt Tolerance, University of California Agricultural Extension Service, E1 Centro, IC-OSA 非 (1974).

22. R.D. Collins, Iowa State University Veterinarian 31 (3) 110 (1969).

23. C.M. Finnel, Imperial County Agricultural Report, Office of Agricultural Commissioner, Imperial County, California (1960-1974).

24. D.W. Cudney, Common Weeds and Herbicides in the Imperial ValZey. University of California Agricultural Extension Service, Imperial County, E1 Centro, Ca1ifornia, Circular 137 (1976). 
25. D.W. Cudney, R.W. Hagemann, T.H. Hinton, D.G. Kontaris, K.S. Mayberry, R.K. Sharma, and A.F. Van Maren, GuideZines to Froduction Coato und Partices Imperial County Crops, Circular 104, University of California Agricultural Extension Service, E1 Centro, California (1975).

26. D.G. Kontaxis, Imperial Agricultural Briefs, Nov. 22, 1974. p.3; Imperial Agricultural Briefs, July 20, 1973, p.4.

27. R.K. Sharma, Imperial Agricultural Briefs, April 19, 1973, p. 6; Implint Agricultural Briefs, Sept. 21, 1973, p. 6; Imperial Agrimultural Bidifa, Dec. 28, 1973, p. 11.

28. H.S. Meister, Imperial Agricultural Briefs, Aug. 29, 1969, p.7; Imonkid. Agricultural Briefs, 0ct. 31, 1969, p. 7; Imperial Agricultural Beicfa, Dec. 26, 1969 p. 7; Imperial Agricultural Briefs, April 17, 1970 p. 6.

29. California Department of Food and Agriculture, Fertizizing Materiala, Oct-Nov-Dec. 1975, Tonnage Report. ACF 58-018. (1976).

30. Ming-Yu Li, Environmental Toxicology, University of California, Davis, private communication, (1976).

31. J.W. Hedgpeth, Arch. Oceanogr. Limnol. (Supp1.) 11, 111 (1959).

32. R.R. Whitney, Contrib. Mar. Sci. 12, 262 (1967).

33. L.H. Carpelan, "Physical and Chemical Characteristics," in The Erology of the Salton Sea, California, in Relation to the Spontfiehing, B.H. Walker, ed. Fish Bulletin No. 113, California Department of Fish and Game, Sacramento, Calif. (1961), p. 17.

34. California Department of Water Resources, Geothermal Hasteo and the Watro Resources of the Salton Sea Area. Bulletin No. 143-7, Sacramento, Calif. (1970).

35. J.R. Spenser, Coachella Valley County Vater District, unpublished data.

36. L.H. Carpelan, "History of the Salton Sea," in The Ecology of" tha Salton Sea, California, in Relation to the Sportfishing, B.W. Walker, Ed. Fish Bulletin No. 113, California Department of Fish and Game, Sacramento (1961), p. 19.

37. L.C. Oglesby, private communcation.

38. L.H. Carpelan, "Phytoplankton and plant productivity," in B.W. Wa1ker op. cit., p. 33.

39. D.R. Young, The Distribution of Cesium, Fubidium, and Potassium in the Quasi-Marine Ecosystem of the Salton Sea, Ph.D. thesis, University Microfilms No. 71-17678. (1970). 
40. L.H. Carpelan, "Zooplankton," in E.W. Walker, op. cit., p. 49.

41. L.H. Carpelan and R.H. Linsley, "The Pile Worm, Neanthes Succinea," in B.W. Walker, op. cit., p. 63.

42. L.J. Hendricks, "The striped Mullet, Mugit cephalus," in B.W. Walker, op. cit., p. 95.

43. B.W. Walker, R.R. Whitney, and G.W. Barlow, "The fishes of the Salton Sea," in B.W. Walker, op. cit., p. 77.

44. L.J. Hendricks, "The threadfin shad, Dorosoma petenense," in B.W. Walker, op. cit., p. 93.

45. J.C. Quast, "The food of the Bairdiella," in B.W. Walker, op. cit., p. 158.

46. R.R. Whitney, "The Bairdiella, Bairdiella icistuis," in B.W. Walker, op. cit., p. 105.

47. R.R. Whitney, "The orangemouth corvina, Cynoscion xanthulus," in B.W. Walker, op. cit., p. 165.

48. U.S. Department of the Interior, Fish and Wildlife Service, Threatened Wizdlife of the United States, 1973 edition.

49. California Department of Fish and Game, At the Crossroads 1976: A Report on California's Endangered and Rare Fish and Wildlife, (January 1976), p. 101.

50. U.S. Department of the Interior, Final Environmental Statement for the Geothermal Leasing Program, Vol. II (1973), p. 300.

51. U.S. Department of the Interior, Salton Sea National Wilalife RefugeCalifornia, Master Plan, Bureau of Sport Fisheries and Wildlife, (June 1971).

52. U.S. Department of the Interior and the Resources Agency of California, Draft Environmental Statement: Salton Sea Project, California, (1974).

53. G. McCaskie, "Shorebird and waterbird use of the Salton Sea," Calif. Fish Game 56 (2), 87 (1970).

54. U.S. Department of the Interior, Final Environmental Statement for the Geothermal Leasing Program, Vol. II. (U.S. Govt. Printing office, Washington, D.C., 1973). 


\section{Section 5 \\ Geothermal Resources}

Donald Ermak and Mary Fiuchanan

\subsection{RESOURCE IDENTIFICATION}

The U.S. Geological Survey has designated a number of areas in the State of California as Known Geothermal Resource Areas (KGRAs). Six KGRAs are located within the Imperial Valley. They are the Brawley, Dunes, East Mesa, Glamis, Heber, and Salton Sea KGRAs. Figure 5.1 shows their location, boundaries, and areas. These areas were designated as KGRAs on the basis of temperature gradient measurements made within the valley. An additional area east of Brawley appears to contain a deep-seated heat source; however, this area has not undergone extensive exploration and has not been designated as a KGRA.

Geothermal resources can be classified into five categories: vapordominated, liquid-dominated, geopressurized, impermeable dry rock, and magma systems. All the geothermal systems in the Imperial Valley are liquid-dominated. The geothermal fluids are 10 to $30 \%$ water vapor by weight when produced, with the remainder of the fluid in the liquid state. The heat energy of the geothermal field is brought to the surface by drilling wells. Generally, after initial stimulation of vell flow, the geothermal fluid flove 110 the vell without additional stimuiation.

Geothermal resources are often divided into two temperature ranges: above $150^{\circ} \mathrm{C}$ and below $150^{\circ} \mathrm{C}$.

Geothermal resources at a tempcrature of about $150^{\circ} \mathrm{C}$ may be considered for generation of electricity, while those below $150^{\circ} \mathrm{C}$ are attractive tor space and process heating. of tho six Iaperial Valley KGRL, four hove resources above $150^{\circ} \mathrm{C}$ : Branley, East Mesa, Heber, and Salton Sat. These areas are also sufficient $1 \mathrm{y}$ large to be of potential economic value. The areas at Dunes and Climl: are probably too small to be economically productive.

Figure 5.1 also shows the rerton. of Inperial county that are irrisited for Egricultural purposes and the bouncaries of the Salton Sea. Threa of the KGRAs (Branley, Heber, and Salton Sea) lie almost entirely within the region of irrigated agriculture. About 10 of the Erit Mesa area is also vithin this ruin... In addition, about $50 \%$ of the silton Sea ligRA is under the salton Sea.

The total land area of tho Imperial Valley LCRAs is 254,827 


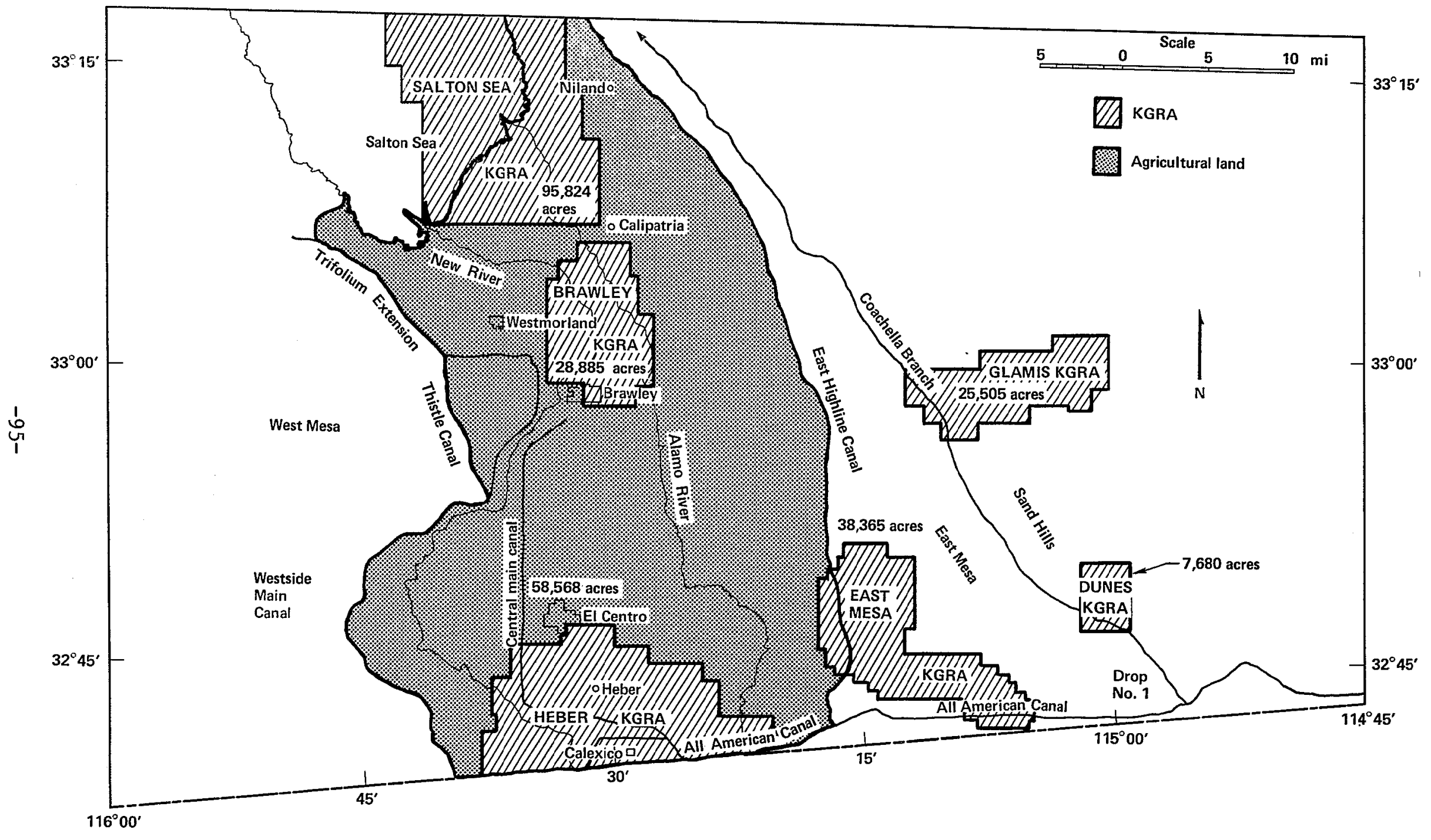

Fig. 5.1. Locations of the six known geothermal resource areas in Imperial Valley. 
acres, which is about $9 \%$ of the

Imperial County land area $(2,942,340$

acres). About 475,000 acres of land

is irrigated for agriculture each

year in the Imperial Valley. of

this land about 140,000 acres

(approximately 30\%) is also designated

as KGRAs. The Salton Sea occupies a

land area of over 200,000 acres.

\subsection{ESTIMATION OF GEOTHERMAL ENERGY RESOURCE}

Evaluations of geothermal resource capacity depend upon knowledge of the reservoir temperature, volume, heat capacity, and water-to-rock ratio. In the Salton Trough there is very little hard data. Towse ${ }^{1}$ estimates that on $1 y 5 \%$ of the resources have been explored by the drilling of wells. Consequently, certain assumptions must be made to estimate the capacity of the resources. In the 1968 to 1972 period, a number of estimates that ranged over 5 orders of magnitude were made of the total heat in storage in the Salton Trough. 2-6 In the last year, several estimates have been made that are all within an order of magnitude of each other. $1,7,8,9$

The estimates of Renner et al., 7 Nathenson and Muffler, ${ }^{9}$ Towse, ${ }^{1}$ and Biehler ${ }^{8}$ are summarized in Table 5.1. Different methods (especially in calculating the reservoir volume) were used to arrive at each set of results.
Renner et al. estimated the total heat stored in the Salton Trough to be $175 \times 10^{18}$ joules $(\mathrm{J})$. Their results were based upon estimates of the reservoir volume to a depth of $10,000 \mathrm{ft}$, reservoir terperatures above $150^{\circ} \mathrm{C}$, and the assumption of a volumetric specific heat of $0.6 \mathrm{cal} / \mathrm{cm}^{3}-{ }^{\circ} \mathrm{C}$. Nathenson and Huffler estimated the recoverable heat of these resources to be $42.5 \therefore 10^{18}$ $J$ by using the figures of Renner et a1. and assuming a net recovery factor of $25 \%$. Recovery factor $1 \mathrm{~s}$ the product of the fraction of rock: that is porous and permeable multiplied by the fraction of thormal energy in the porous permeable part that is recoverable. They used a value of 0.5 for these two factors and on $1 y$ considered resources with a temperature greater than $150^{\circ} \mathrm{C}$. The Towse estimate is $20 \because 10^{18} \mathrm{~J}$ and considers only geothermal fluids at a temperature of $230^{\circ} \mathrm{C}$ or greater. He used the temperature gradient maps of Combs ${ }^{3}$ to calculate the reservotr volume. Usable geothermal fluid was assumed to extend $1,000 \mathrm{ft}$ below the $230^{\circ} \mathrm{C}$ isothermal surface or to a maximum depth of $7,000 \mathrm{ft}$, whichever is lesis. To complete the calculation, he assumed a well head enthalpy of between 1.0 to $1.3 \approx 10^{6} \mathrm{~J} / \mathrm{kg}$, a specilic yield of 0.16 (specific yield = fluid volume/reservoir volume), and a fluid density of 1 
Table 5.1. Estimates of the stored heat in the geothermal resources of Imperial Valley.

\begin{tabular}{|c|c|c|c|c|}
\hline & $\mathrm{A}$ & $\underset{\mathrm{B}}{\text { Stored heat }}\left(10^{18}\right.$ & $\begin{array}{l}\mathrm{J}) \\
\mathrm{C}\end{array}$ & $\mathrm{D}$ \\
\hline Salton Sea & 87.9 & 22.0 & 11.0 & 31.2 \\
\hline Heber & 46.0 & 11.5 & 3.5 & 12.5 \\
\hline East Mesa & 23.0 & 5.8 & 3.0 & 15.6 \\
\hline Brawley & 12.6 & 3.2 & 1.0 & 19.8 \\
\hline Glamis & 1.7 & - & 1.0 & - \\
\hline Dunes & 2.5 & - & 0.5 & 0.8 \\
\hline E. Brawley & 0.8 & - & - & - \\
\hline Total & 174.5 & 42.5 & 20.0 & 79.1 \\
\hline
\end{tabular}

$A=$ Total heat in rock and water. ${ }^{7}$

$B=$ Heat in geothermal resource. (Data of Renner et $\underline{\text { al }} .{ }^{7}$ were used and a specific yield of 0.25 was assumed.)

$\mathrm{C}=$ Heat in geothermal resource. (Specific yield of 0.16 assumed. ${ }^{1}$ )

$\mathrm{D}=$ Heat in geothermal resource. (Specific yield of 0.16 assumed.) Three estimates in the ratio 0.67 to 1 to 2 were made. The middle estimate is listed. 8

$\mathrm{gm} / \mathrm{cm}^{3}$. Biehler's estimates range

from $53 \times 10^{18}$ to $160 \times 10^{18} \mathrm{~J}$.

Using Bouger gravity maps, he calculated

the reservoir volume on the basis of

the residual gravity anomalies

associated with each geothermal

reservoir. He also assumed a specific

yield of 0.16 , an enthalpy of $1.3 \times$

$10^{6} \mathrm{~J} / \mathrm{kg}$ and a fluid density of $1 \mathrm{~g} / \mathrm{cm}^{3}$.

A11 estimates shown in Table 5.1 are within less than 10 times of each other with the Towse value being the smallest and the Renner value being the largest. Renner and Towse estimate that 50 to $55 \%$ of the heat in storage is associated with the Salton Sea field. The Biehler estimate for the Salton Sea area is about $40 \%$ of the total for the Salton Trough. Only the Salton Sea, Heber, East Mesa, and Brawley fields are expected to be economical on a commercial basis. The other fields are too small or are too low in temperature. 
Production of electrical energy is accomplished by using the high temperature, high pressure geothermal fluid to either directly or indirectly turn a turbine that then runs an electric generator. Not all of the heat energy in the geothermal fluid can be converted to mechanical energy in the turbine. For steam turbines, the fraction of convertible energy depends upon the input and output temperature of the steam. To maximize this fraction, the input temperature must be as high as possible and the output temperature as low as possible. For a geothermal plant, the input temperature cannot be higher than the temperature of the geothermal fluid. Depending upon the temperature of the geothermal fluid, efficiencies from 8 to $18 \%$ can be expected from geothermal plants. In contrast, modern fossil fuel plants operate at efficiencies of about $36 \%$ by superheating the steam $\left(540^{\circ} \mathrm{C}\right)$ to be used in the turbine.

Estimates of the potential electrical energy production by Nathenson and Muffler ${ }^{9}$ and those of Towse ${ }^{1}$ are given in Table 5.2. Nathenson and Muffler considered conversion to electrical power by the flashed steam process and only considered fluids above $150^{\circ} \mathrm{C}$. They used the following conversion efficiencies: 150 to $200^{\circ} \mathrm{C}$, $\mathrm{e}=0.08 ; 200$ to $250^{\circ} \mathrm{C}$, e $=0.10$; and 250 to $300^{\circ} \mathrm{C}$, e $=0.12$. Touse con- sidered several geothermal power technologies: binary, flashed steam, and total flow. The minimum resource temperature considered was $230^{\circ} \mathrm{C}$. Tho conversion efficiencies used by Towac were those of Austin et a1. ${ }^{10}$ The efficiencies shown in Table 5.2 for Towse's data are the average values calculated from his estimated geothermal heat energy and his estimated clocm trical energy production. Dosplte the different assumptions nade by these. authors, the final estinates for the total potential for electric cnergy production of the Salton Basin region are in good agreement. They are $4580 \mathrm{HN}$ (Nathenson $\&$ luffeler ${ }^{9}$ ) and $3350 \mathrm{NJ}$ (Towse ${ }^{1}$ ) for a $30-y r$ period.

\subsection{FHYSICAL AND CHEUICAL CHARACTERIZATION OF THE (EEOTHERHAL FLUIDS}

The physical and chemical characteristics of the geothemal fluids determine its potential for conversion to electric power, the type of conversion technology that can be used (e.g., binary, total flow, etc.), the expected electrical power, and the potential hazards to the environment through the emission of gaseous and liquid wastes. While some data exist for each of the KGRAs, in general the information is quite scanty. Most of the information that has been collected to date is for the Salton Sea and East Mesa 
Table 5.2. Estimated electrical energy production, 30-yr equivalent - MW.

\begin{tabular}{|c|c|c|c|c|c|}
\hline & \multicolumn{3}{|c|}{ Reference 9} & \multicolumn{2}{|c|}{ Reference 1} \\
\hline & e & $\mathrm{T}$ & $P$ & $\mathrm{e}$ & $\mathrm{P}$ \\
\hline Sa1ton Sea & 0.12 & 340 & 2787 & 0.164 & 2000 . \\
\hline Heber & 0.08 & 190 & 973. & \multicolumn{2}{|c|}{ A11 others: } \\
\hline East Mesa & 0.08 & 180 & 487. & 0.12 & 1350 \\
\hline Brawley & 0.10 & 200 & 333. & & \\
\hline Glamis/Dunes/E. Brawley & & 135 & 0 . & & \\
\hline Total & & & 4580 . & & 3350 . \\
\hline
\end{tabular}

e = Average electrical energy conversion efficiency.

$\mathrm{T}=$ Geothermal resource temperature ${ }^{\circ} \mathrm{C}$.

$\mathrm{P}=$ Electrical power in MW for 30-yr period.

KGRAs. Palmer ${ }^{11}$ and Hoffman ${ }^{12}$ each present the characteristics of about 20 geothermal wells located in the Salton Sea field. The characteristics of six East Mesa wells are given by the Bureau of Reclamation in a 1974 Status report: 13

Geothermal Reservoir Temperature and Pressure

Renner et al. ${ }^{7}$ give representative temperatures for each of the KGRAs in the Imperial Valley (see Table 5.2). On the basis of geothermal fluid temperature, the KGRAs can be graded in decreasing order as Salton Sea, Brawley, Heber, East Mesa, Dunes, and Glamis. The average well bottom temperature of 16 Salton Sea wells taken from Palmer ${ }^{11}$ and Hoffman ${ }^{12}$ is $286 \pm 45^{\circ} \mathrm{C}$. This value for the average temperature of the salton Sea KGRA is considerably less than the value used by Renner et a1., 7 (Table 5.2), but still leaves the Salton Sea field as the hottest in the valley. The average temperature of 6 East Mesa wells is $180 \pm 13^{\circ} \mathrm{C}$. This result is in very good agreement with the temperature used by Renner et al.

Figure 5.2 presents the temperature and hydrostatic pressure profiles of the Bureau of Reclamation's Mesa 8-1 geothermal well. The profiles increase in both temperature and pressure with increased well depth as is typical of geothermal wells. 


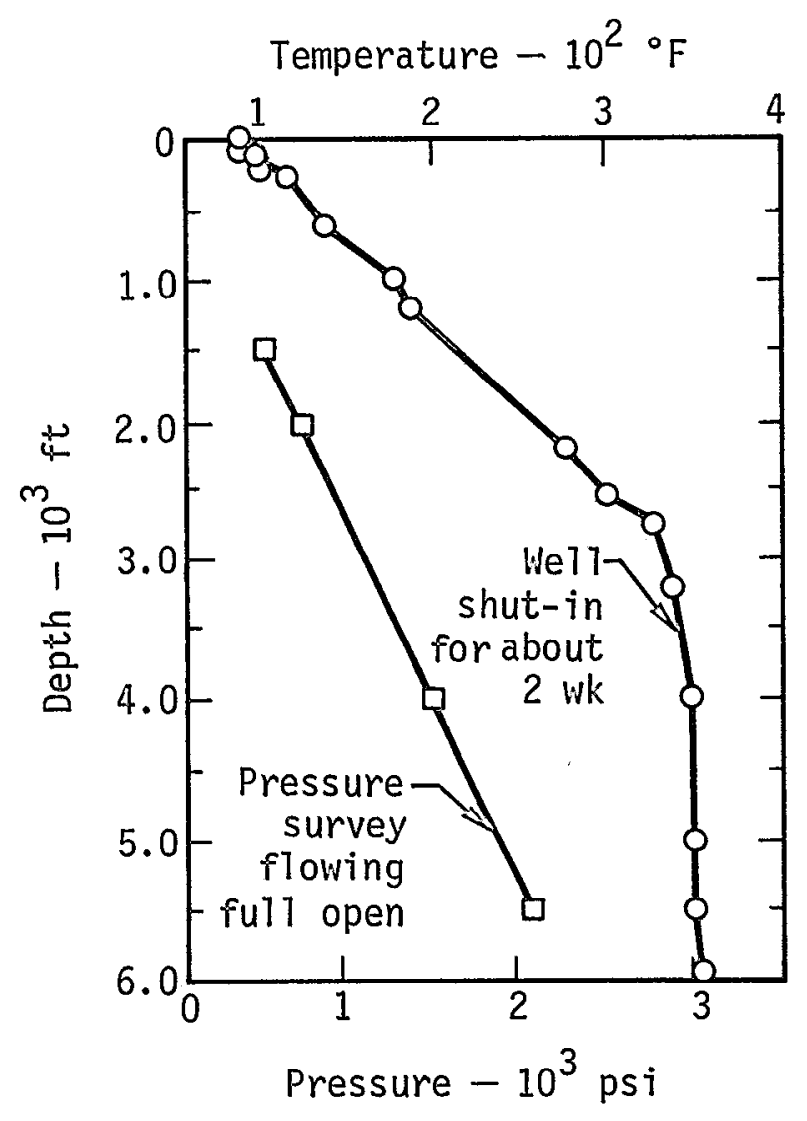

Fig. 5.2. Temperature and pressure profiles for the Mesa 8-1 well, Imperial Valley, California, May $29,1974.13$

The hydrostatic pressure at a depth of $6,000 \mathrm{ft}$ is about 2,600 pounds per square inch (psi) in the Salton Sea field and about 2,300 psi in the East Mesa field. Based upon data from six wells, Helgeson ${ }^{5}$ indicates that fluid pressures in the Salton Sea field are normal hydrostatic pressures. Presumably this is true throughout the Imperial Valley. 10 The pressure profile of six Salton Sea wells and two East Mesa wells is given in Fig. 5.3 with a curve for pure water as a comparison.
Physical Characteristics at the Vo11 Head

Production rates of geotherma1 wells depend upon the well-head pressure, well-orifice size, and duration of flow. The well-head temperature and flow rate as a function of we11-head pressure are given in Fig. 5.4 for two East Mesa wells. As the we:L1-head pressure is increased the well-head temperature increascs; however, the flow rate decreases. The fraction of geothermal fluid that is ste.am ranges from 10 to $20 \%$. Using thermodynamic considerations, the thəoretical available energy from the Hesa 6-1 well is $3.4 \mathrm{MH}$.

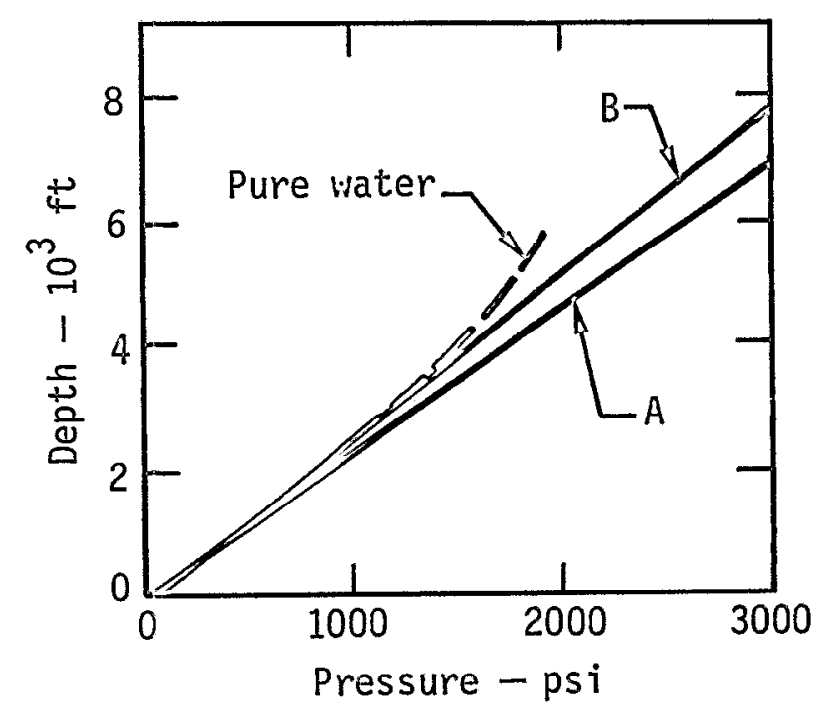

Fig. 5.3. Pressure-depth profiles for selected geothermal Wells, 13September 1974. A = Salton Sea wells, hydrostatic gradient = 0.384 psi/ft; $B=$ East Mesa wells, hydrostatic gradient $=0.434$ psi/ft.10,13 

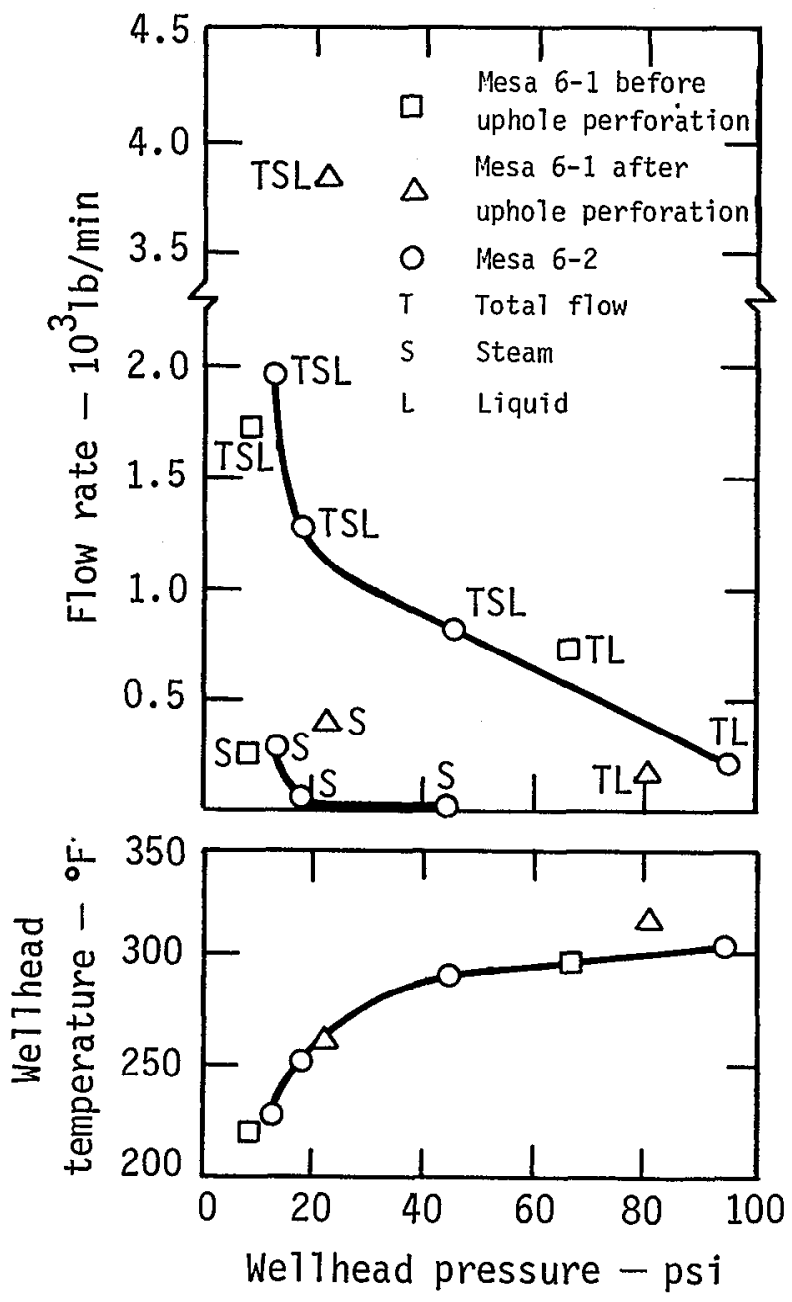

Fig. 5.4. Temperature, pressure, and flow rates for Mesa $6-1$ and Mesa 6-2 wel1s, Imperial Valley, California. 13

Flow rates for wells in the Salton Sea KGRA are quite high. Palmer ${ }^{11}$ presents production characteristics for 10 wells in the Salton Sea field. The average flow rate for these wells is $435,000 \mathrm{lb} / \mathrm{hr}$ at an average pressure of $215 \mathrm{psi}$ and an average of $19 \%$ steam by weight. The fraction of steam ranged from 10 to $25 \%$. Well orifice size for the 10 wells ranged from 5 1/2-to 8 -in diameter. The average fluid enthalpy of 10 Salton Sea wells characterized by Hoffman ${ }^{12}$ is $242 \pm 26$ calories/gram $(\mathrm{cal} / \mathrm{g})$. Helgeson ${ }^{5}$ reports that several Salton Sea wells have produced for up to 18 months without appreciable decrease in the flow rate, temperature, and enthalpy.

\section{Chemical Composition of the Brines}

The usefulness of a liquiddominated geothermal resource depends on the salinity of the fluid, its thermal properties, and on the ability to bring the fluid to the surface. Fluids that have a high salt content will cause severe corrosion and scaling problems in the wells and support equipment. These problems, if severe enough, will require costly maintenance expenses and can make a power plant uneconomical to operate.

The geothermal fluids in the Imperial Valley KGRAs are generally quite saline. There appears to be a salinity gradient such that the saIinity is lowest near the Colorado River side of the valley and increases northwestward toward the Salton Sea. The total dissolved solids (TDS) in the Salton Sea KGRA averages about 210,000 ppm. In the East Mesa KGRA, TDS averages about 2,100 ppm and in the Heber KGRA about $20,000 \mathrm{ppm}$. For comparison, the salinity of sea water is about $33,000 \mathrm{ppm}$ and the salinity of the Salton sea is 39,000 ppm TDS. 
Table 5.3 summarizes the chemical composition data for geothermal brine collected for the wells in the Salton Sea and East Mesa KGRAs. For some constituents, the standard deviation is as large or larger than the average concentration, indicating a large variance from well to well. In addition, the constituent concentration for a single well often varied by 25 to $50 \%$ when measured at different times. One well in the East Mesa that had a TDS content 10 times higher. than the other wells was loft out of the statistics since it is not considered to be representative of the field. 14

The brines of the Salton Sca KGRA have extremely high salinity (about 10 times that of sea mater). Consequently, while the Salton Sca KGRA is estimated to contain about $50 \%$ of the total geothermal resource in the Imperial Valley, it also has the highest salinity. The problems of corrosion and scaling must be overcome before these fluids can be

Table 5.3. Geothermal brine composition of Salton Sea and East Mesa rells.

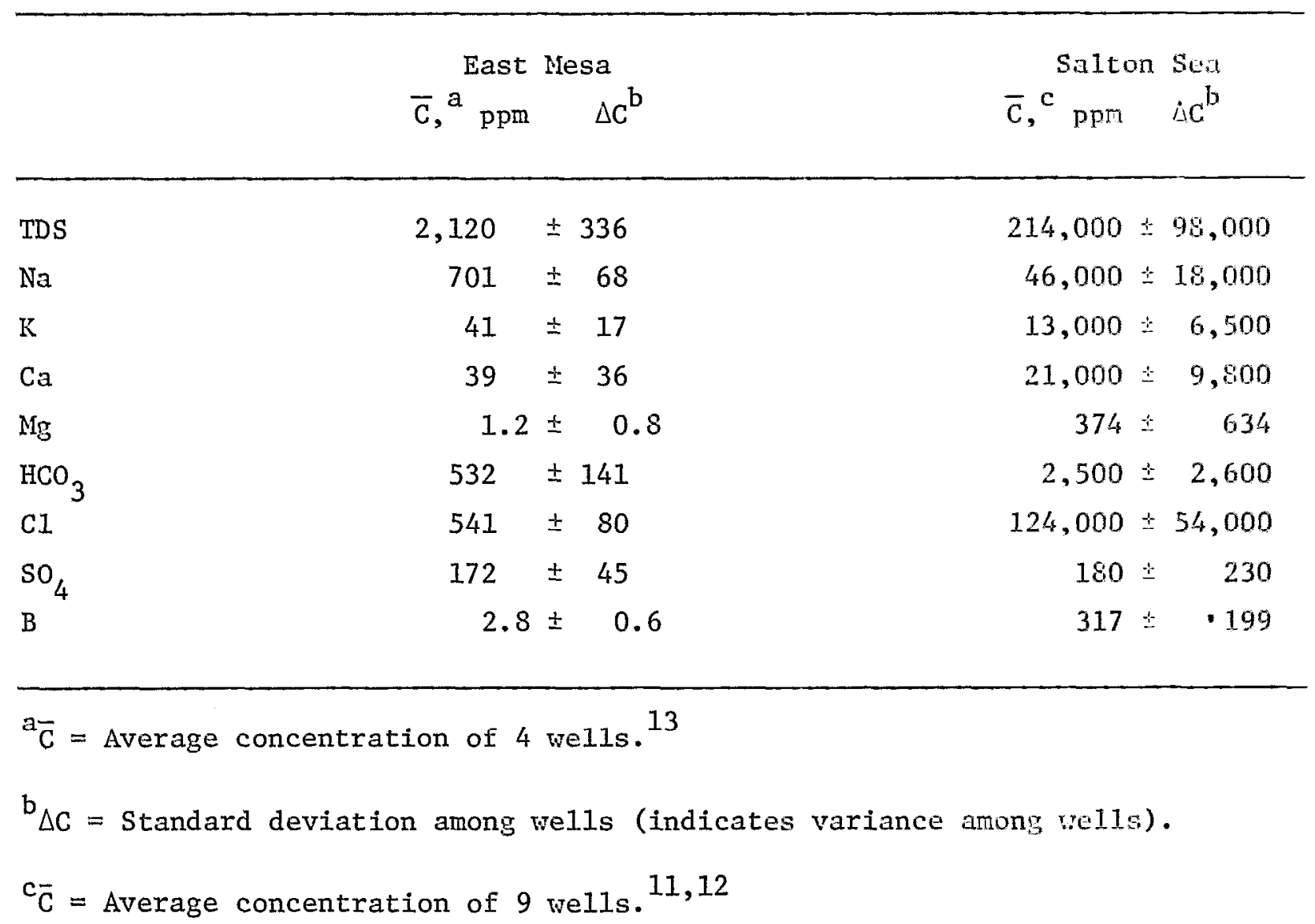


used economically for the production of electrical energy.

\section{Noncondensable Gases}

A small fraction of the geothermal fluids is composed of noncondensable gases that are ultimately emitted to the atmosphere during the conversion from geothermal heat energy to electrical energy. The fraction of noncondensable gases in the Imperial Valley resources is estimated to be about $1 \% .^{15}$ While the composition of this gas fraction is highly variable, $\mathrm{CO}_{2}$ is always the major fraction with lesser amounts of $\mathrm{H}_{2} \mathrm{~S}, \mathrm{H}_{2}, \mathrm{CH}_{4}, \mathrm{NH}_{3}$, and $\mathrm{N}_{2} \cdot{ }^{16}$ Even though $\mathrm{CO}_{2}$ constitutes the major fraction of the noncondensable gas emitted from a geothermal plant, the emissions of $\mathrm{CO}_{2}$ from an equivalent fossil-fuel plant are about 20 times higher. ${ }^{17}$

There are to date no known measurements of the noncondensable gas fraction from the KGRAs in the Imperial Valley. As a crude indicator, the emissions at the Geysers geothermal plant in Sonoma County, California are given in Table 5.4. The Geysers plant is a vapor-dominated system and presently products 502 MWe. The fraction of emitted steam that is noncondensable gas is $0.4 \%$ by weight. Also listed in Table 5.4 are the atmospheric emissions of solids in cooling tower drift.
The geothermal emission of major concern is $\mathrm{H}_{2} \mathrm{~S}$ with possibly $\mathrm{NH}_{3}$ as a distant second. Hydrogen sulfide is toxic at high concentrations, but these high levels are not expected to occur as a result of geothermal operations. The problem is odor. The California State standard to prevent odor from $\mathrm{H}_{2} \mathrm{~S}$ is $0.03 \mathrm{ppm}$, which is $42 \mu \mathrm{g} / \mathrm{m}^{3}$. The odor threshold for ammonia is 46.8 $\mathrm{ppm}$, which is about $35,000 \mu \mathrm{g} / \mathrm{m}^{3}$. The odor of hydrogen sulfide has been detected at the Geysers. At Cerro Prieto, which lies in the southern part of the Salton Trough in Mexico, Axtmann ${ }^{19}$ estimates the $\mathrm{H}_{2} \mathrm{~S}$ emissions to be 15 times higher than at the Geysers. In addition to the odor problem, $\mathrm{H}_{2} \mathrm{~S}$ is believed to be converted in the atmosphere to $\mathrm{SO}_{2}$ and sulfate compounds.

\subsection{OVERVIEW OF GEOTHERMAL DEVELOPMENT}

The development of Imperial Valley's geothermal resources has been characterized by years of efforts thwarted by insufficient interest and funds, as well as underdeveloped technology, Early exploratory drilling recovered $\mathrm{CO}_{2}$ and potash primarily as commercially usable by-products. Problems resulting from drilling highly saline brine made initial geothermal drilling 
Table 5.4. Noncondensable gas and solid emissions at the Geysers geothermal plant. ${ }^{a}$

\begin{tabular}{lccc} 
& \multicolumn{2}{c}{ Noncondensable gases } & Solids \\
& $\mathrm{kg} / \mathrm{MN} \cdot \mathrm{hr}$ & Percent & $\mathrm{kg} / \mathrm{MH} \cdot \mathrm{yr}$ \\
$\mathrm{CO}_{2}$ & 25.93 & 81.9 & \\
$\mathrm{H}_{2} \mathrm{~S}$ & 1.80 & 5.7 & \\
$\mathrm{NH}_{3}$ & 1.54 & 4.8 & \\
$\mathrm{CH}_{4}$ & 1.54 & 4.8 & \\
$\mathrm{H}_{2}$ & 0.45 & $\mathrm{I.4}$ & \\
$\mathrm{N}_{2}$ & 0.42 & 1.3 & 0.017 \\
$\mathrm{As}$ & & & 0.001 \\
$\mathrm{~B}$ & & & \\
$\mathrm{Hg}$ & & & \\
\hline
\end{tabular}

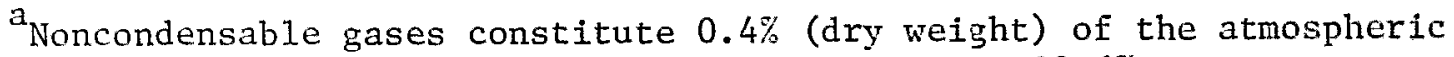
emissions with steam constituting the remaining $99.6 \%$.

${ }^{b}$ Percent of the noncondensable gas fraction.

sites either economically unfeasible or at hest short-1ived undertakings. It was finally the unproductive wildcat tests for oil and natural gas that fostered enough interest in site studies, equipment improvements, and drilling techniques to insure the support of the private, industrial, and governmental sectors necessary to fully explore the geothermal potential of the valley. Table 5.5 summarizes the significant drillings and studies that have taken place in the valley. ${ }^{20}$ current and on-gaing operations are shown in Tabl€: 5.6 . 
Table 5.5. Significant drillings and studies conducted in Imperial Valley.

Year Location $\quad$ Organization $\begin{gathered}\text { Well depth and type } \\ \text { of findings }\end{gathered}$

\begin{tabular}{|c|c|c|c|}
\hline 1927 & $\begin{array}{l}\text { Mullet Island } \\
\text { (Sa1ton Sea area) }\end{array}$ & $\begin{array}{l}\text { Pioneer Development } \\
\text { Company }\end{array}$ & $\begin{aligned} & 449 \mathrm{~m}- \text { Steam, carbon } \\
& \text { dioxide and hot } \\
& \text { water }\end{aligned}$ \\
\hline
\end{tabular}

1934- Mullet Island

1954 vicinity
150-200 m - Carbon dioxide and hot water
We11 abandoned; steam lacked sufficient pressure for power generation.

$\stackrel{1}{\stackrel{1}{0}}$

\section{Mullet Island vicinity}

\section{7- Niland}

1958

\section{Salton Sea area}

Joseph I. O'Neill, Jr.

\section{Kent Imperial} Corporation

$$
1400 \mathrm{~m}-600^{\circ} \mathrm{F} \text { Brine }
$$

$1440 \mathrm{~m}$ - Hot water and steam $600^{\circ} \mathrm{F}$

$1441 \mathrm{~m}$ - Steam and high salinity water
Approximately 60 wells recovered $\mathrm{CO}_{2}$ from shallow sands containing hot water (as high as $60^{\circ} \mathrm{C}$ ). $\Lambda$ commercial dry ice plant that had operated at the site from 1932 to 1954 was shut down and well drilling discontinued when rising waters of the Salton Sea began to inundate the field.

Wildcat test for oil and natural. gas was unsuccessful.

Sinclair \#1 well produced steam intermittently for 4 months before the highly saline and corrosive brines encountered in the area forced a shut down when the well scaled up at the surface.

Sportsman \#1 we11 was a good steam producer, but had highly saline brine flows. 
Table 5-5. (Continued)

\begin{tabular}{ccc}
\hline Year Location & Organization & $\begin{array}{c}\text { Well depth and type } \\
\text { of findings }\end{array}$
\end{tabular}

1962 Salton Sea area

1963 Brawley vicinity

1965

占

1968 Salton Sea area
Joseph I. O'Neil1, $\mathrm{Jr}$.

5232 ft - Steam and brine $207^{\circ} \mathrm{C}$

$4097 \mathrm{~m}$ - Hot water and steam California

Imperial Therma1

Products, Inc.

(subsidiary of Morton

International) and

Earth Energy Company

(subsidiary of Union

Pure Oil Co.)

University of California at Riverside (UCR) (supported by U.S. Bureau of

Reclamation,

National Science

Foundation (NSF)

and others)
Hypersaline brine caused scaling and corrosion problems.

oil exploration unsuccessful but prompted more extensive geothermal research in area.

Plants were primarily designed to recover potash from brine; however, a drastic drop in the price of this mineral and scaling problems made the projects economically unfeasible.

Study located several KGRAs and other geophysical data. Also estimated that recoverable water in storage was in the magnitude of 1 billion acre-feet. 
Table 5-5. (Continued)

$\begin{array}{ccc}\text { Year Location } & \text { Well depth and type } \\ \text { of findings }\end{array}$

1968 Imperial Valley UCR

\begin{tabular}{|c|c|}
\hline 1972 & $\begin{array}{l}\text { Salton Sea - } \\
\text { Imperial Valley }\end{array}$ \\
\hline
\end{tabular}

1973- Imperial Valley1974 Salton Sea area
California Division of $0 i 1$ and Gas

(Funded by National

Geodetic Survey,

NSF and others)

San Diego Gas \&

Electric Co.

(SDG\&E), Phillips

Petroleum, Lawrence

Livermore Laboratory

(LLL), Chevron Oil Co.,

U.S. Bureau of

Reclamation
Discovered four and possibly five new high heat flow areas, excluding the already known geothermal field in the Buttes area. The high-heat flow areas were named the North Brawley, Heber, Dunes, and Mesa. A moderate anomaly was discovered east of Brawley and designated the east Brawley anomaly.

12 wells were drilled in the Salton Trough, 3 in Salton Sea area, 3 on the Heber anomaly, 1 on the East Mesa anomaly, and $I$ on the Dunes anomaly. A subsidence surveillance network in the Imperial Valley and adjacent lands was also established.

12 geothermal wells were drilled in the Imperial Valley: 4 in the centra valley, 4 at East Mesa, 1 in the Salton Sea area, and 3 in the Heber area. 
Table 5.6. Geothermal research and development projects in Tmperia1 Valley.

\begin{tabular}{|c|c|c|c|c|}
\hline Source funding & Type project & $\begin{array}{l}\text { Performing } \\
\text { organizations (s) }\end{array}$ & $\begin{array}{l}\text { Principal } \\
\text { investigator (s) }\end{array}$ & Research and development \\
\hline $\begin{array}{l}\text { National Science } \\
\text { Foundation (NSF) }\end{array}$ & $\begin{array}{l}\mathrm{H}_{2} \mathrm{~S} \\
\text { effects }\end{array}$ & $\begin{array}{l}\text { University of Cali- } \\
\text { fornia, Riverside } \\
\text { (UCR) }\end{array}$ & C. Ray Thompson & $\begin{array}{l}\text { Behavior of } \mathrm{H}_{2} \mathrm{~S} \text { in the } \\
\text { atmosphere and its effects } \\
\text { on vegetation. }\end{array}$ \\
\hline NSF & $\begin{array}{l}\text { Computer } \\
\text { simulation }\end{array}$ & $\begin{array}{l}\text { Systems, Science } \\
\text { and Software }\end{array}$ & $\begin{array}{l}\text { John Pritchett and } \\
\text { Larry Rice }\end{array}$ & A reservoir modeling study. \\
\hline NSF & $\begin{array}{l}\text { Geothermal } \\
\text { metals }\end{array}$ & Battelle Northwest & David E. Robertson & $\begin{array}{l}\text { An investigation of the } \\
\text { nature and environmental } \\
\text { aspects of heavy metals } \\
\text { released during geothermal } \\
\text { energy development. }\end{array}$ \\
\hline NSF & $\begin{array}{l}\text { Noise } \\
\text { effects }\end{array}$ & $\begin{array}{l}\text { St. Mary's College, } \\
\text { Dept. of Biology, } \\
\text { Moraga, Ca. }\end{array}$ & Phillip Leitner & $\begin{array}{l}\text { The environmental effects of } \\
\text { noise (on animals) from } \\
\text { geothermal rcsource development. }\end{array}$ \\
\hline NSF & $\begin{array}{l}\text { Trace } \\
\text { elements }\end{array}$ & $\begin{array}{l}\text { University of } \\
\text { Southern Califor- } \\
\text { nia, Environmental } \\
\text { Engineering Program. }\end{array}$ & Kenneth Chen & $\begin{array}{l}\text { Study of trace elements found } \\
\text { in geothermal wastewaters. }\end{array}$ \\
\hline NSF & Geological & UCR & Wilfred Elders & $\begin{array}{l}\text { A geological study of the } \\
\text { Salton Sea Anomaly. }\end{array}$ \\
\hline NSF & $\begin{array}{l}\text { Geothermal } \\
\text { development } \\
\text { planning }\end{array}$ & & $\begin{array}{l}\text { Dayid E. Pierson } \\
\text { et al }\end{array}$ & $\begin{array}{l}\text { The Imperial County Geothermal } \\
\text { Element Study. }\end{array}$ \\
\hline
\end{tabular}


Table 5-6. (Continued)

Source funding Type project $\begin{gathered}\text { Performing } \\ \text { organization(s) }\end{gathered} \quad \begin{gathered}\text { Principal } \\ \text { investigator(s) }\end{gathered} \quad$ Research and development

$\overline{\text { NSF }}$

Energy Research and Development Agency (ERDA) in cooperation with industry, local, state and other federal agencies

ERDA

San Diego Gas \&

Magma Power Co., and ERDA

$\begin{array}{ll}\text { ERDA and } & \text { Pilot } \\ \text { Southern } & \text { plant } \\ \text { California } & \\ \text { Edison } & \end{array}$

\section{Systems Control Inc., E. John Finnemore Palo Alto, CA.}

Environmental effects

Lawrence Livermore Laboratory (LLL)

Lynn Anspaugh and Paul Phelps

Arthur L. (Roy) Austin

SDG\&E and Magma Power Co.

Southern California Edison, Phillips Petroleum, and Southern Pacific Land Co.

\begin{abstract}
Geothermal
development
\end{abstract}

LLL James Nugent and
Robert Lacey

J. Lynn Rasband, Craig Racine, and William Berge

\section{Subsidence study.}

An environmental baseline data and integrated assessment study.
Studies on geothermal technology development. Most of the research program is based on the total flow concept and scale and corrosion control.

A proposed 10-MW pilot plant operation in the Salton Sea anomaly. The primary interest is in the development of a binary cycle system for power generation.

A proposed 10-MW pilot plant operation in the Sinclair tract. The main goals are continuous and reliable production of geothermal brine, development of suitable reinjection systems, and controlling the problem of scaling and corrosion. 
Table 5-6. (Continued)

\begin{tabular}{|c|c|c|c|c|}
\hline Source funding & Type project & $\begin{array}{l}\text { Performing } \\
\text { organization(s) }\end{array}$ & $\begin{array}{l}\text { Principal } \\
\text { investigator (s) }\end{array}$ & Research and development \\
\hline $\begin{array}{l}\text { U.S. Bureau of } \\
\text { Reclamation }\end{array}$ & Desalination & $\begin{array}{l}\text { U.S. Bureau of } \\
\text { Reclamation, ERDA, } \\
\text { Bechtel and TRH }\end{array}$ & $\begin{array}{l}\text { Manuel Lopez and } \\
\text { M. K. Fulcher }\end{array}$ & $\begin{array}{l}\text { A desalination demonstration } \\
\text { project taking place in the East } \\
\text { Mesa as part of continuing } \\
\text { operations. }\end{array}$ \\
\hline ERDA & Design Work & TRW Corporation & $\begin{array}{l}\text { Robert Douglas and } \\
\text { Joseph Kennedy }\end{array}$ & $\begin{array}{l}\text { Hardware test facility in East } \\
\text { Mesa area. }\end{array}$ \\
\hline ERDA & $\begin{array}{l}\text { Equipment } \\
\text { study }\end{array}$ & Sperry-Sun & Warren McBee & $\begin{array}{l}\text { Test of Sperry-Sun down-hole } \\
\text { pump at Heber. }\end{array}$ \\
\hline ERDA & Socioeconomic & $\begin{array}{l}\text { Battelle Memorial } \\
\text { Institute, Human } \\
\text { Affairs Research } \\
\text { Center }\end{array}$ & $\begin{array}{l}\text { C. Richard Schuller } \\
\text { and Roland Cole }\end{array}$ & $\begin{array}{l}\text { A study of the legal institutional } \\
\text { and political problems confronting } \\
\text { geothermal development in } \\
\text { California. }\end{array}$ \\
\hline ERDA & Economics & Bechtel Corp. & Jerry H. Hankin & $\begin{array}{l}\text { Conceptual design and capital cost } \\
\text { estimates for two 50-1W plants. }\end{array}$ \\
\hline $\begin{array}{l}\text { ERDA and } \\
\text { California } \\
\text { Energy } \\
\text { Commission }\end{array}$ & $\begin{array}{l}\text { Regional } \\
\text { planning } \\
\text { project }\end{array}$ & $\begin{array}{l}\text { Jet Propulsion } \\
\text { Laboratory (JPL), } \\
\text { Pasadena, Ca. and } \\
\text { Stanford Research } \\
\text { Institute (SRI), } \\
\text { Palo Alto, Ca. }\end{array}$ & $\begin{array}{l}\text { Richard Maullin } \\
\text { and Charles } \\
\text { Frederickson }\end{array}$ & $\begin{array}{l}\text { An assessment of geothermal } \\
\text { resources in California with } \\
\text { consideration of the various } \\
\text { implications of energy development. }\end{array}$ \\
\hline $\begin{array}{l}\text { U.S. Bureau } \\
\text { of Reclamation }\end{array}$ & $\begin{array}{l}\text { Reservoir } \\
\text { study }\end{array}$ & Inter-Comp & Larry Rice & \\
\hline
\end{tabular}


Table 5-6. (Continued)

Source funding Type project $\begin{gathered}\text { Performing } \\ \text { organization(s) }\end{gathered} \begin{gathered}\text { Principal } \\ \text { investigator(s) }\end{gathered} \quad$ Research and development

$\begin{array}{lll}\text { Environmental } & \text { Environmental } & \text { Don Gilmore } \\ \text { effects } & \text { Protection Agency } & \\ & \text { (EPA), Las Vegas }\end{array}$

ERDA

Data base

Lawrence Berkeley Laboratory (LBL)

Sidney Phillips

ERDA in cooperation with U.S.

Bureau of

1 Reclamation

ERDA

ERDA and State

(California)

Energy Resource

Conservation

and Development

Commission

ERDA

U.S. Bureau of Reclamation
Test

facility

Test

facility

Computer

simulation

Socio-

economic

Desalination

Consultants

Bechtel Corp.

TRW, LBL
U.S. Bureau of Reclamation and LBL

Jet Propulsion

Laboratory (JPL)

and Stanford Research

Institute (SRI)

Isabel Reiff and Mike Franfel

Jerry N. Hankin, Leon Awerback, Thomas Lindemuth, and Emile Houle
Assesses the environmental

impact of extraction, conversion, and waste disposal of geothermal systems.

Project seeks to establish a National Geothermal Information

Resource (both library and computer based).

Development of a geothermal test facility at the East Mesa Test Facility.

This is a continuation of test facility development at the East Mesa Test Facility.

Operations research/system analysis planning for geothermal development.

Study of socioeconomic aspects of geothermal development.

Two experimantal desalination plants operating in the East Mesa are available to researchers studying the characteristics of geothermal fluids. Each unit is designed to produce 75 to $190 \mathrm{kl}$ of distilled water per day. 
Table 5-6. (Continued)

\begin{tabular}{|c|c|c|c|c|}
\hline Source funding & Type project & $\begin{array}{l}\text { Performing } \\
\text { organization (s) }\end{array}$ & $\begin{array}{l}\text { Principal } \\
\text { investigator (s) }\end{array}$ & Research and development \\
\hline $\begin{array}{l}\text { Electric Power } \\
\text { Research } \\
\text { Institute } \\
\text { (EPRI) }\end{array}$ & $\begin{array}{l}\text { Feasibility } \\
\text { study }\end{array}$ & $\begin{array}{l}\text { Ben Holt, ProCon, } \\
\text { Inc., and Geonomics, } \\
\text { Inc. (Subcontractors) }\end{array}$ & $\begin{array}{l}\text { Vasel Roberts, } \\
\text { Phillip La Mori, } \\
\text { Charles Best, George } \\
\text { Wiegele, Tsvi } \\
\text { Meidav, and Mae } \\
\text { Meidav }\end{array}$ & $\begin{array}{l}\text { A study of the effects and } \\
\text { feasibility of developing } \\
\text { geothermal resources in the } \\
\text { Heber anomaly. }\end{array}$ \\
\hline $\begin{array}{l}\text { EPRI and } \\
\text { SDG\&E }\end{array}$ & $\begin{array}{l}\text { Environmental } \\
\text { effects }\end{array}$ & $\begin{array}{l}\text { Envi Con, etc. } \\
\text { (Subcontractors) }\end{array}$ & $\begin{array}{l}\text { Vase1 Roberts } \\
\text { and Phillip La Hori }\end{array}$ & $\begin{array}{l}\text { Baseline environmental study } \\
\text { in the Heber area. }\end{array}$ \\
\hline $\begin{array}{l}\text { Republic Geo- } \\
\text { thermal, Inc. } \\
\text { and City of } \\
\text { Burbank }\end{array}$ & $\begin{array}{l}\text { Geothermal } \\
\text { exploration }\end{array}$ & $\begin{array}{l}\text { Republic Geothermal, } \\
\text { Inc. }\end{array}$ & $\begin{array}{l}\text { Robert Rex, Tim } \\
\text { Evans, and } \\
\text { Martindale Kile }\end{array}$ & Exploration program. \\
\hline $\begin{array}{l}\text { Magma Power, } \\
\text { Inc. }\end{array}$ & $\begin{array}{l}\text { Geothermal } \\
\text { exploration } \\
\text { and development }\end{array}$ & Magma Power, Inc. & Thomas Hindrichs & $\begin{array}{l}\text { Continuing exploration activities as } \\
\text { well as the drilling of two wells in } \\
\text { the East Mesa anomaly area. Planning } \\
\text { started on a 10-MN pilot plant }\end{array}$ \\
\hline Union $0 i 1$ Co. & $\begin{array}{l}\text { Geothermal } \\
\text { exploration }\end{array}$ & $\begin{array}{l}\text { Union 0i1 Co. of } \\
\text { California }\end{array}$ & $\begin{array}{l}\text { Care1 otte and } \\
\text { Anthony } \mathrm{J} . \text { Chasteen }\end{array}$ & $\begin{array}{l}\text { Exploration drilling in the } \\
\text { Brawley and Heber anomalies. }\end{array}$ \\
\hline $\begin{array}{l}\text { Geothermal Energy } \\
\text { Systems, Inc. }\end{array}$ & $\begin{array}{l}\text { Equipment } \\
\text { study }\end{array}$ & $\begin{array}{l}\text { Energy Systems Inc., } \\
\text { Nerport Beach, Ca. }\end{array}$ & Ray Rodde & $\begin{array}{l}\text { Project to develop a doin-hole } \\
\text { heat exchanger. }\end{array}$ \\
\hline
\end{tabular}


Table 5-6. (Continued)

Source funding Type project $\begin{gathered}\text { Performing } \\ \text { organization(s) }\end{gathered} \quad \begin{gathered}\text { Principal } \\ \text { investigator(s) }\end{gathered}$ Research and development

\begin{tabular}{|c|c|c|c|c|}
\hline $\begin{array}{l}\text { SDG\&E (in } \\
\text { relation to } \\
\text { Palo Verde Sun }\end{array}$ & $\begin{array}{l}\text { Equipment } \\
\text { study }\end{array}$ & SDG\&E & James Nugent & $\begin{array}{l}\text { Transmission corridor study } \\
\text { that may have application to } \\
\text { geothermal power transmission. }\end{array}$ \\
\hline
\end{tabular}

Desert nuclear

\section{Californta}

Division of $\mathrm{Oil}$

and Gas, LLL,

USGS and other

$\stackrel{\stackrel{\omega}{\omega}}{\stackrel{\omega}{\omega}}$

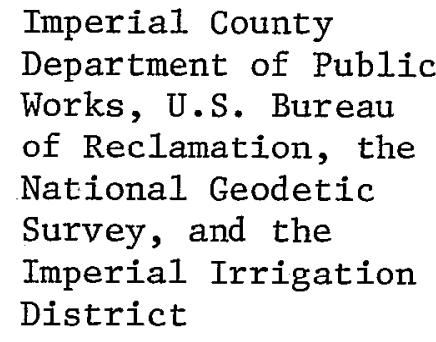

Imperial County Department of Public Works, U.S. Bureau of Reclamation, the National Geodetic Survey, and the Imperial Irrigation District

UCR

USGS and CIT

Seismo-

logical

Institute of

Technology (CIT)

with partial

funding from LLL

ERDA/LLL

U.S. Bureau of Reclamation

networks

U.S. Bureau of

Reclamation
David Estes, Ben

Lofgren, and

Harold Ganow

Shawn Biehler

Gary Fuis

Neil Crow and

Paul Kasameyer

Kim Mathias
Valley wide

An on-going program that monitors the subsidence survey network.

Salton Sea area

Salton Sea area

East Mesa 
Table 5-6. (Continued)

\begin{tabular}{|c|c|c|c|c|}
\hline Source funding & Type project & $\begin{array}{l}\text { Performing } \\
\text { organization(s) }\end{array}$ & $\begin{array}{c}\text { Principal } \\
\text { investigator (s) }\end{array}$ & Research and development \\
\hline
\end{tabular}

\begin{tabular}{|c|c|c|c|c|}
\hline USGS & Geology & $\begin{array}{l}\text { USGS, Menlo Park, } \\
\text { Ca. }\end{array}$ & $\begin{array}{l}\text { L.J.P. Muffler } \\
\text { and Ben Lofgren }\end{array}$ & Geologic research program. \\
\hline $\begin{array}{l}\text { Bob Bonds \& } \\
\text { Assoc. }\end{array}$ & $\begin{array}{l}\text { Resource } \\
\text { investigation }\end{array}$ & $\begin{array}{l}\text { Bob Bonds \& Assoc., } \\
\text { Montgomery, Texas }\end{array}$ & H.R. Bond & $\begin{array}{l}\text { Study designed to determine } \\
\text { feasibility of mineral extraction } \\
\text { from geothermal brines. }\end{array}$ \\
\hline $\begin{array}{l}\text { Morton- } \\
\text { Norwich }\end{array}$ & $\begin{array}{l}\text { Resource } \\
\text { investigation }\end{array}$ & $\begin{array}{l}\text { Imperial Thermal } \\
\text { Products }\end{array}$ & Charles Yeater & $\begin{array}{l}\text { Feasibility studies on mineral } \\
\text { extraction from geothermal brines. }\end{array}$ \\
\hline $\begin{array}{l}\text { EPRI and } \\
\text { State (Cali- } \\
\text { fornia) Energy } \\
\text { Commission }\end{array}$ & Equipment study & $\begin{array}{l}\text { Ben Holt, Inc. } \\
\text { (Subcontractors: } \\
\text { Pro-con, } \\
\text { Geonomics) }\end{array}$ & Ed Ghormley & $\begin{array}{l}\text { Heat exchanger test at the } \\
\text { Chevron site at Heber. }\end{array}$ \\
\hline ERDA & $\begin{array}{l}\text { Feasibility } \\
\text { study }\end{array}$ & TRH and Holly Sugar & Russ Pierson & $\begin{array}{l}\text { Study to determine how geothermal } \\
\text { Energy can bc uscd in the Holly } \\
\text { Sugar Plant. }\end{array}$ \\
\hline ERDA & $\begin{array}{l}\text { Feasibility } \\
\text { study }\end{array}$ & $\begin{array}{l}\text { Valley Nitrogen } \\
\text { Products, Uest Tech } \\
\text { Services }\end{array}$ & Bill Johnson & $\begin{array}{l}\text { Research on direct heat utiliza- } \\
\text { tion by Valley Nitrogen Products. }\end{array}$ \\
\hline
\end{tabular}




\section{REFERENCES}

1. D. Towse, An Estimate of the Geothermal Energy Resource in the Salton Trough, California, Lawrence Livermore Laboratory, Rept. UCRL-5185I (1975).

2. D.N. Anderson and L.H. Axte11, "Geothermal Resources in California," in Geothermal Overviews of the Westem United States, (Geothermal Resources Council, Davis, California, Special Rept. No. 1, 1972).

3. J. Combs, "Heat Flow and Geothermal Resource Estimates for the Imperial Valley," in Cooperative Geophysical-Geochemical Investigations of California (University of California, Riverside, 1971).

4. L.C. Dutcher, W.F. Hardt, and W.R. Moyle, Jr., PreZiminary Appraisal of Ground Water in Storage with Reference to Geothermal Resources in the Impexial Valley Area, California, U.S. Geological Survey, Washington, D.C., Circular 649 (1972).

5. H.C. Helgeson, Amer. J. Sci., 226, 129 (1968).

6. R.W. Rex, "Investigation of Geothermal Resources in the Imperial Valley and their Potential Value for Desalination of Water and Electricity Production," in Compendium of Papers Presented at the Imperial ValleySalton Area Geothermal Hearing (State of California Geothermal Resources Board, Sacramento, 1970).

7. J.L. Renner, D.E. White, and D.L. Williams, "Hydrothermal Convection Systems," in Assessment of Geothermal Resources of the United States, U.S. Geological Survey, Circ. 726 (1975).

8. S. Biehler, A Preliminary Report on a Resource Assessment of the Imperial VaZley. Part I, University of California, Riverside, Rept. IGPP-UCRL-76-8 (1976).

9. M. Nathenson and L.J.P. Muffler, "Geothermal Resources in Hydrothermal Convection Systems and Conduction-Dominated Areas," in Assessment of Geothermal Resources of the United States, U.S. Geological Survey, Circ. 726 (1975).

10. A.L. Austin, G.H. Higgins, and J.H. Howard, The Total Flow Concept for Recovery of Energy from Geothermal Hot Brine Deposits, Lawrence Livermore Laboratory, Rept. UCRL-51366 (1973).

11. T.D. Palmer, Characteristics of Geothermal Wells Located in the Salton Sea Geothermal Field, Imperial County, California, Lawrence Livermore Laboratory, Rept. UCRL-51976 (1975). 
12. M.R. Hoffman, Brine Chemistry-Sealing and Corrosion, Environmental Quality Laboratory, Memorandum No. 14 (1975).

13. Geothermal Resource Investigations, East Mesa Test Site, Imperial Valley, California, U.S. Bureau of Reclamation, Status Report (November 1974).

14. K. Mathias, U.S. Bureau of Reclamation, private communication (May 18, 1976).

15. J.B. Koenig, "Worldwide Status of Geothermal Resources Development," in Geothermal Energy, P. Kruger and C. Otte, Eds. (Stanford University Press, 1973).

16. 0.H. Krikorian, "Corrosion and Scaling in Nuclear-Stimulated Geotherma1 Power Plants," in Geothermal Energy, P. Kruger and C. Otte, Eds. (Stanford University Press, 1973).

17. R.G. Bowen, "Environmental Impact of Geothermal Development," in Geothemult Energy, P. Kruger and C. Otte, Eds. (Stanfard University Press, 1973).

18. Pacific Gas \& Electric Company, Amended Enrironmental Lata Statement, Geysers 13, (1975).

19. R.C. Axtmann, "Emission Control of Gas Effluents from Ceothermal Power Plants," Environ. Lett. 8, 136 (1975).

20. T.D. Palmer, J.H. Howard, and D.P. Lande, Geothemal Development of the Salton Trough, Califomia and Mexico, Lawrence Livermore Laboratory, Rept. UCRL-51775 (1975). 


\section{Section 6 \\ County Economic Characterization}

Kenda11 Haven

\subsection{COUNTY ECONOMIC COMPOSITION:}

The economy of Imperial County has been dominated by agricultural activities since the early 1900 's. The sectors currently active in the county economy are described in California County Business Patterns ${ }^{1}$ collected annually by the U.S. Department of Commerce for employment for payrol1, and more recently by Lofting ${ }^{2}$ for sector dollar transactions in a county input/output (I/O) mode1. The County Business Patterns gives activities by Standard Industrial Codes (SIC's) but include only those activities covered by state employee compensation coverage. Thus, most agricultural activities, railroads, and governmental activities are not included. These are very significant omissions for Imperial County, since agricultural and governmental (federal, state, and 1ocal) activities are two of the largest sectors in the county for both payroll and employment. The principal value of the County Business Patterns data is in analyzing county labor force distributions, sector employment potentials, mean salary levels, etc.

The Lofting I/O model, showing dollar flows between economic business sectors, can be used to analyze the infrastructure of the county economy to predict overal1 changes in the economy resulting from changes in any one sector. The sector designations in this model do not correspond directly to the SIC's in County Business Patterns and, in general, direct comparison of data in the $I / O$ model and County Business Patterns is not possible. Such direct comparison can only be made at the level of major economic divisions (manufacturing, wholesale, etc.). Finally, the $I / O$ model does include government and agriculture, which is subdivided into eight subsectors (ffeld crops, fruits and vegetables, beef cattle and livestock, etc.).

Neither the I/0 model nor the complete County Business Pattern tables are reproduced in this report. Table 6.1 lists County Business Patterns. payroll and employment data for Imperial County by SIC. Employment data collected by Lofting in support of his model development are given where significant discrepancies exist between his and the data from County Business Patterns. These discrepancies bordering on two different sectors are assigned under different sector headings. Additional discrepancies can develop as a result of the differing 
Table 6.1. Imperial County sector employment and payrol1 for $1972 .^{1}$

\begin{tabular}{|c|c|c|c|c|c|}
\hline \multirow[b]{2}{*}{$\begin{array}{l}\text { SIC } \\
\text { number }\end{array}$} & \multirow[b]{2}{*}{ Sector name } & \multicolumn{2}{|c|}{ Employment $^{a}$} & \multicolumn{2}{|c|}{ Payrol1 } \\
\hline & & $\frac{\text { County }}{\frac{\text { Business }}{\text { Patterns }} 1}$ & $\begin{array}{l}\text { Data, } 2 \\
\text { Lofting }\end{array}$ & $\begin{array}{l}\text { First } \\
\text { quarter } \\
\text { payrol1 } \\
1972-\$ \mathrm{~K}\end{array}$ & $\begin{array}{l}\text { Pay/ } \\
\text { cmployeo } \\
\text { ratio }\end{array}$ \\
\hline 07 & $\begin{array}{l}\text { Agricultural } \\
\text { services, forestry, } \\
\text { fishing }\end{array}$ & 681 & & 1651 & 2.42 \\
\hline 0700 & $\begin{array}{l}\text { Agricultural } \\
\text { services and hunting }\end{array}$ & 681 & & 1651 & 2.42 \\
\hline 0710 & $\begin{array}{l}\text { Miscellaneous } \\
\text { agricultural } \\
\text { services }\end{array}$ & 488 & & 1333 & 2.73 \\
\hline 0720 & Animal husbandry & 176 & & 310 & 1.76 \\
\hline 10 & Mining & 16 & & 15 & 0.94 \\
\hline 15 & $\begin{array}{l}\text { Contract } \\
\text { construction }\end{array}$ & 620 & & 1243 & 2.00 \\
\hline 1500 & $\begin{array}{l}\text { General building } \\
\text { construction }\end{array}$ & 153 & & 238 & 1.56 \\
\hline 1600 & Heavy construction & 151 & & 306 & 2.03 \\
\hline 1700 & Special trade & 316 & & 699 & 2.21 \\
\hline 1710 & $\begin{array}{l}\text { Plumbing, heating, } \\
\text { air conditioning }\end{array}$ & 75 & & 175 & 2.33 \\
\hline 1730 & Electrical & 45 & & 107 & 2.38 \\
\hline 19 & Manufacturing & 1475 & 1508 & 2776 & 1.99 \\
\hline 2000 & Food and kindred & 490 & & 940 & 1.92 \\
\hline 2040 & Grain mills & 165 & 189 & 284 & 1.72 \\
\hline 2042 & $\begin{array}{l}\text { Prepared feed for } \\
\text { animals }\end{array}$ & 165 & & 283 & 1.72 \\
\hline 2060 & Sugar & 139 & & 280 & 2.01 \\
\hline 2063 & Beet sugar & 139 & & 280 & 2.01 \\
\hline 2300 & $\begin{array}{l}\text { Appare1, other } \\
\text { textiles }\end{array}$ & 34 & & 42 & 1.24 \\
\hline 2400 & Lumber and wood & 136 & & 228 & 1.68 \\
\hline 2700 & $\begin{array}{l}\text { Printing and } \\
\text { publishing }\end{array}$ & 150 & & 216 & 1.44 \\
\hline 2710 & Newspapers & 122 & & 175 & 1.43 \\
\hline 2800 & $\begin{array}{l}\text { Chemical and allied } \\
\text { manufacturing }\end{array}$ & 108 & 130 & 374 & 2.88 \\
\hline
\end{tabular}

${ }^{a}$ Enployment in subcategories will not total to one-digit SIC because only certain subcategories (two-, three-, and four-digit SIC) are listed. 


\begin{tabular}{|c|c|c|c|c|c|}
\hline \multirow[b]{2}{*}{$\begin{array}{l}\text { SIC } \\
\text { number }\end{array}$} & \multirow[b]{2}{*}{ Sector name } & \multicolumn{2}{|c|}{ Employment ${ }^{a}$} & \multicolumn{2}{|c|}{ Payro 11} \\
\hline & & $\begin{array}{l}\frac{\text { County }}{\text { Business }} \\
\text { Patterns }\end{array}$ & $\begin{array}{l}\text { Data, } \\
\text { Lofting }\end{array}$ & $\begin{array}{l}\text { First } \\
\text { quarter } \\
\text { payrol1 } \\
1972-\$ \mathrm{~K}\end{array}$ & $\begin{array}{l}\text { Pay/ } \\
\text { employee } \\
\text { ratio }\end{array}$ \\
\hline 2870 & $\begin{array}{l}\text { Agricultural } \\
\text { chemicals }\end{array}$ & 103 & 130 & 296 & 2.87 \\
\hline 3200 & $\begin{array}{l}\text { Stone, clay and } \\
\text { glass manufacturing }\end{array}$ & 451 & & 1041 & 2.31 \\
\hline 3270 & Concrete, plaster & 451 & & 1041 & 2.31 \\
\hline 3273 & Ready mix & 139 & & 320 & 2.30 \\
\hline 3275 & Gypsum products & 273 & & 630 & 2.31 \\
\hline 3500 & $\begin{array}{l}\text { Machinery except } \\
\text { electrical }\end{array}$ & 58 & & 123 & 2.12 \\
\hline 3900 & $\begin{array}{l}\text { Misce1laneous } \\
\text { manufacturing }\end{array}$ & 48 & & 89 & 1.85 \\
\hline 40 & $\begin{array}{l}\text { Transportation and } \\
\text { public utilities }\end{array}$ & 2042 & & 1733 & 1.96 \\
\hline 4100 & $\begin{array}{l}\text { Local and interurban } \\
\text { transportation }\end{array}$ & 117 & & 211 & 1.80 \\
\hline 4200 & $\begin{array}{l}\text { Trucking and } \\
\text { warehousing }\end{array}$ & 351 & & 697 & 1.99 \\
\hline 4210 & Trucking & 294 & & 600 & 2.04 \\
\hline 4211 & $\begin{array}{l}\text { Trucking without } \\
\text { storage }\end{array}$ & 289 & & 589 & 2.04 \\
\hline 4700 & $\begin{array}{l}\text { Transportation } \\
\text { services }\end{array}$ & 20 & & 35 & 1.75 \\
\hline 4800 & Communications & 283 & & 531 & 1.88 \\
\hline 4810 & Telephone & 218 & & 409 & 1.88 \\
\hline 4900 & $\begin{array}{l}\text { Electricity, gas, } \\
\text { and sanitary } \\
\text { utilities }\end{array}$ & 1271 & & 3519 & 2.77 \\
\hline 4910 & Electrical utilities & 100 & & 297 & 2.97 \\
\hline 4950 & Sanitary services & 32 & & 72 & 2.25 \\
\hline 4970 & Irrigation systems & 1139 & & 1563 & 1.37 \\
\hline 50 & Wholesale & 1422 & & 2469 & 1.74 \\
\hline 5040 & $\begin{array}{l}\text { Groceries and } \\
\text { related }\end{array}$ & 558 & & 1026 & 1.84 \\
\hline
\end{tabular}




\begin{tabular}{|c|c|c|c|c|c|}
\hline \multirow[b]{2}{*}{$\begin{array}{l}\text { SIC } \\
\text { number }\end{array}$} & \multirow[b]{2}{*}{ Sector name } & \multicolumn{2}{|c|}{ Emp loyment ${ }^{a}$} & \multicolumn{2}{|c|}{ Payrol1 } \\
\hline & & $\frac{\frac{\text { County }}{\text { Business }}}{\text { Patterns }}$ & $\begin{array}{l}\text { Data, } \\
\text { Lofting }\end{array}$ & $\begin{array}{l}\text { First } \\
\text { quarter } \\
\text { payroll } \\
1972-\$ \mathrm{~F}\end{array}$ & $\begin{array}{l}\text { Pay/ } \\
\text { employce } \\
\text { ratio }\end{array}$ \\
\hline 5048 & $\begin{array}{l}\text { Fresh fruit and } \\
\text { vegetables }\end{array}$ & 464 & & 828 & 1.78 \\
\hline 5050 & $\begin{array}{l}\text { Farm products - } \\
\text { raw materials }\end{array}$ & 240 & & 212 & 0.88 \\
\hline 5052 & Cotton & 74 & & 65 & 0.88 \\
\hline 5090 & $\begin{array}{l}\text { Miscellaneous food } \\
\text { and kindred } \\
\text { manufacturing }\end{array}$ & 383 & & 782 & 2.04 \\
\hline 5092 & Petroleum products & 61 & & 101 & 1.66 \\
\hline 5099 & $\begin{array}{l}\text { Miscellaneous } \\
\text { manufacturing }\end{array}$ & 135 & & 285 & 2.11 \\
\hline 52 & Retail trade & 4586 & 4611 & 5690 & 1.25 \\
\hline 5200 & $\begin{array}{l}\text { Building materials } \\
\text { and farm equipment }\end{array}$ & 313 & 356 & 531 & 1.70 \\
\hline 5250 & $\begin{array}{l}\text { Hardware and } \\
\text { farm equipment }\end{array}$ & 210 & & 331 & 1.58 \\
\hline 5251 & Hardware stores & 106 & & 167 & 1.58 \\
\hline 5252 & $\begin{array}{l}\text { Farm equipment } \\
\text { dealers }\end{array}$ & 104 & & 163 & 1.57 \\
\hline 5300 & General retail & 600 & & 659 & 1.10 \\
\hline 5310 & Department stores & 236 & & 289 & 1.20 \\
\hline 5330 & Variety stores & 172 & & 137 & 0.80 \\
\hline 5390 & $\begin{array}{l}\text { Miscellaneous } \\
\text { general retail }\end{array}$ & 157 & & 162 & 1.03 \\
\hline 5400 & Food and kindred & 686 & & 1124 & 1.64 \\
\hline 5410 & Grocery stores & 605 & & 1011 & 1.67 \\
\hline 5500 & $\begin{array}{l}\text { Auto and } \\
\text { auto services }\end{array}$ & 985 & & 1458 & 1.48 \\
\hline 5510 & $\begin{array}{l}\text { New and used } \\
\text { car dealers }\end{array}$ & 419 & & 772 & 1.84 \\
\hline 5530 & $\begin{array}{l}\text { Tires, batteries, } \\
\text { and accessories }\end{array}$ & 164 & & 278 & 1.70 \\
\hline 5540 & Gas station services & 372 & & 380 & 1.02 \\
\hline
\end{tabular}


Table 6.1. (Continued)

\begin{tabular}{|c|c|c|c|c|c|}
\hline \multirow[b]{2}{*}{$\begin{array}{l}\text { SIC } \\
\text { number }\end{array}$} & \multirow[b]{2}{*}{ Sector name } & \multicolumn{2}{|c|}{ Employment ${ }^{a}$} & \multicolumn{2}{|c|}{ Payro11 } \\
\hline & & $\frac{\frac{\text { County }}{\text { Business }}}{\text { Patterns }}$ & $\begin{array}{l}\text { Data, } 2 \\
\text { Lofting }\end{array}$ & $\begin{array}{l}\text { First } \\
\text { quarter } \\
\text { payrol1 } \\
1972-\$ \mathrm{~K}\end{array}$ & $\begin{array}{l}\text { Pay/ } \\
\text { employee } \\
\text { ratio }\end{array}$ \\
\hline 5600 & $\begin{array}{l}\text { Appare1 and } \\
\text { accessories }\end{array}$ & 432 & & 395 & 0.91 \\
\hline 5620 & $\begin{array}{l}\text { Women's } \\
\text { ready-to-wear }\end{array}$ & 157 & & 126 & 0.80 \\
\hline 5650 & Family clothes & 201 & & 181 & 0.90 \\
\hline 5700 & Furniture & 125 & & 177 & 1.42 \\
\hline 5800 & $\begin{array}{l}\text { Eating and drinking } \\
\text { places }\end{array}$ & 910 & & 637 & 0.70 \\
\hline 5900 & Miscellaneous retail & 517 & & 683 & 1.23 \\
\hline 5910 & Drug stores & 197 & & 306 & 1.55 \\
\hline 5920 & Liquor stores & 68 & & 60 & 0.88 \\
\hline 5930 & Antique stores & 32 & & 37 & 1.16 \\
\hline 5960 & Farm and garden & 84 & & 141 & 1.68 \\
\hline 5990 & Miscellaneous retail & 53 & & 42 & 0.79 \\
\hline 60 & $\begin{array}{l}\text { Finance, insurance, } \\
\text { real estate }\end{array}$ & 624 & & 1055 & 1.69 \\
\hline 6000 & Banking & 310 & & 594 & 1.92 \\
\hline 6020 & Commercia1 banking & 310 & & 594 & 1.92 \\
\hline 6100 & Credit agencies & 84 & & 146 & 1.74 \\
\hline 6140 & Personal credit & 52 & & 90 & 1.73 \\
\hline 6400 & Insurance & 58 & & 83 & 1.00 \\
\hline 6500 & Real estate & 87 & & 78 & 0.90 \\
\hline 6510 & $\begin{array}{l}\text { Real estate } \\
\text { operator and lessor }\end{array}$ & 71 & & 66 & 0.93 \\
\hline 70 & Services & 2050 & & 2462 & 1.20 \\
\hline 7000 & $\begin{array}{l}\text { Hotel and } \\
\text { other lodging }\end{array}$ & 244 & & 257 & 0.75 \\
\hline 7010 & Hotel, motel & 326 & & 242 & 0.74 \\
\hline 7200 & Personal services & 280 & & 309 & 1.10 \\
\hline 7210 & Laundries & 143 & & 156 & 1.09 \\
\hline 7230 & Beauty shops & 98 & & 90 & 0.92 \\
\hline
\end{tabular}




\begin{tabular}{|c|c|c|c|c|c|}
\hline \multirow[b]{2}{*}{$\begin{array}{l}\text { SIC } \\
\text { number }\end{array}$} & \multirow[b]{2}{*}{ Sector name } & \multicolumn{2}{|c|}{ Employment ${ }^{a}$} & \multicolumn{2}{|c|}{ Payrol1 } \\
\hline & & $\frac{\frac{\text { County }}{\text { Business }}}{\text { Patterns }} 1$ & $\begin{array}{l}\text { Data, } 2 \\
\text { Lofting }\end{array}$ & $\begin{array}{l}\text { First } \\
\text { quarter } \\
\text { payrol1 } \\
1972-\$ \mathrm{~K}\end{array}$ & $\begin{array}{l}\text { Payl } \\
\text { eriployed } \\
\text { rat lo }\end{array}$ \\
\hline 7300 & $\begin{array}{l}\text { Miscellaneous } \\
\text { business services }\end{array}$ & 184 & & 239 & 1.30 \\
\hline 7390 & $\begin{array}{l}\text { Miscellaneous } \\
\text { business services }\end{array}$ & 113 & & 163 & 1.44 \\
\hline 7500 & $\begin{array}{l}\text { Auto repair and } \\
\text { services }\end{array}$ & 126 & & 157 & 1.25 \\
\hline 7530 & Auto repair & 104 & & 143 & 1.38 \\
\hline 7538 & General auto repair & 53 & & 73 & 1.38 \\
\hline 7600 & $\begin{array}{l}\text { Miscellaneous auto } \\
\text { repair }\end{array}$ & 80 & & 120 & 1.50 \\
\hline 7690 & $\begin{array}{l}\text { Miscellaneous auto } \\
\text { repair shops }\end{array}$ & 41 & & 65 & 1.59 \\
\hline 7900 & $\begin{array}{l}\text { Amusement and } \\
\text { recreation }\end{array}$ & 89 & & 87 & 0.98 \\
\hline 8000 & $\begin{array}{l}\text { Medical and other } \\
\text { health }\end{array}$ & 359 & & 560 & 1.56 \\
\hline 8010 & Offices of doctors & 204 & & 367 & 1.80 \\
\hline 8020 & office of dentists & 54 & & 58 & 1.07 \\
\hline 8100 & Legal services & 52 & & 87 & 1.62 \\
\hline 8600 & $\begin{array}{l}\text { Nonprofit } \\
\text { organizations }\end{array}$ & 261 & & 267 & 1.02 \\
\hline 8640 & $\begin{array}{l}\text { Civic and social } \\
\text { groups }\end{array}$ & 62 & & 34 & 0.55 \\
\hline 8660 & Religious services & 34 & & 18 & 0.53 \\
\hline 8690 & N.E.C. services & 103 & & 141 & 1.37 \\
\hline 8900 & $\begin{array}{l}\text { Miscellaneous } \\
\text { services }\end{array}$ & 107 & & 216 & 2.02 \\
\hline 8930 & Accounts auditing & 51 & & 102 & 2.00 \\
\hline 99 & Unclassified & 119 & & 115 & 0.97 \\
\hline 100 & Al1 agriculture & 6840 & & 4537 & 0.71 \\
\hline Total & & $13635^{\mathrm{b}}$ & & 19210 & 1.55 \\
\hline
\end{tabular}

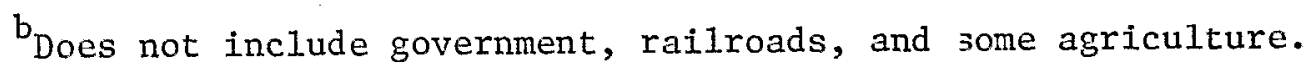


methodologies used for filling in information omitted from the data by the Department of Commerce because of federal disclosure laws. The data in Table 6.1 serve as a basic description of economic activities in Imperial County and their relative magnitude and importance; some analysis of this information is provided in section 6.5 of this report. A more detailed analysis of agriculture is given in sections 6.2 and 6.3 which deal with agriculture and county employment, respectively.

\section{Economic Sector Description}

The data in Table 6.1 provide some interesting insights into the Imperial County economy. First, manufacturing employment is very low in Imperial County, and the county is slightly below state average for construction. These are two relatively high paying economic sectors as indicated by their respective pay/employee ratios. Agricultural services (SIC-07) has the highest pay/employee ratio of the major economic divisions, while the highest sector ratio is in chemical manufacturing (SIC-2800). Imperial County is one of the state centers for SIC-07 activity and is near average in sector 2800 .

Relatively high paying local activities increase the circulation of monies within the local economy by increasing household sector purchases. Lower paying jobs tend to result in a concentration of household expenditures in a narrower range of basic goods, while higher pay results in a wider diversification of household spending and, as a result, increased employment in more diverse wholesale, retail, and service sectors. Manufacturing activity is very important to a local economy because manufactured products tend to be exported outside the local area. These export activities (basic sectors) such as agricultural and manufacturing activities bring new money into the local economy. Nonbasic activities (wholesale, retail, services, etc.) circulate and redistribute money within the local economy but tend not to generate new money. The data in Table 6.1 illustrates that basic sectors tend to have higher pay/employee ratios than nonbasic sectors. The principal exception is agriculture, which traditionally has paid low wages.

Table 6.2 lists the major sectoral omissions in the 1972 employment data that are now part of Imperial County's economy. Several of these industries $(1300,1381$, 1382) are ássociated with geothermal activity. Since 1972, several new industrial activities have located 
Table 6.2. Major sectors absent from the Imperial County employment data in 1972.
SIC number
Industry title

1300

1381

1382

2010

2030

2200

2600

3400

3520

3530

8060

8200
Oil and gas extraction

Drilling wells

Exploration services

Meat products nanufacturing

Canned cured and frozen food manufacturing

Textile mill products

Paper and allied products manufacturing

Fabricated metal manufacturing

Farm machinery manufacturing

Construction machinery manufacturing

Hospitals

Education services in Imperial Valley and are listed in Table 6.3. These listings reduce Table 6.2 omissions by several sectors. While some of the major sectors not found in the Imperial County economy can be significant paths for the export of dollars out of a local economy (i.e., SIC's 2010, 2030, 2600 and 3520), the list is small for a county with a population of approximately 83,000. Further analysis of the economic position of Imperial County is included in section 6.4 of this report.

\section{Multiplier Effects}

A sector multiplier measures the total dollar amount of economic activity that will be generated in a local economy by a $\$ 1$ increase in external demand for a product of that local economy. If a demand is generated outside of the county econony (as represented by an input/ output model) for a product of that econoriy (agricultural products, geothermal energy, Salton Sca recreation, etc.) then the I/O mode1 tells us that the increase in activity in that one sector will also cause increased activity in other sectors. A multiplier measures this total generated activity. Lofting's I/O model has been used to generate various multipliers for each sector in the Imperial County economy. 
Table 6.3. New industrial activities in Imperial County (1972-1974). a

Company

Estimated

Location

jobs

1. Anthony Williams Inc.

Clothing manufacturing

60

E1 Centro

2. A. \& J. Manufacturing Company

Golf equipment manufacturing

40

E1 Centro

3. Chem Fab Corporation

Chemical milling processing

30

Imperial

4. 3 J Manufacturing Company

Clothing manufacturing

100

Calexico

5. Ameron Steel

Steel products (Under construction)

Imperial

6. E1 Centro Garment Manufacturing Inc.

150

E1 Centro

7. Kona Kai (Under construction)

100

Calexico

8. Suzy Manufacturing Company (Under

construction)

Curtain manufacturing

150

Calexico

9. Imperial Yarn \& Needle

Manufacturing mops

65

Calexico

10. Earley's Textiles Center Inc.

35

Ho1tville

11. Dune Buggy Enterprises

Assemblying dune buggies

8

Brawley

12. R. R. Ornamental Design

Wrought iron - fabricators

3

Brawley

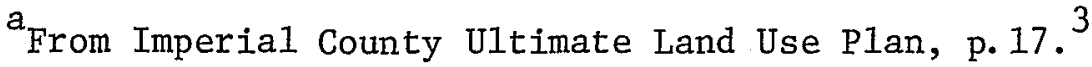

(See Tab1es $6.4 \mathrm{a}, 6.4 \mathrm{~b}, 6.4 \mathrm{c}$, and 6.4d.)

A11 multipliers in Tables 6.4a-d are for both direct and indirect (total) effects of the increase in sector unit demand. In explanation of the column headings of Table 6.4a, an output multiplier represents the total number of dollars generated in the local economy by. a dollar increase in sector final demand, employment multiplier is the change in employment per million dollars of sector output generated by a unit increase in sector final demand, and value added multipliers measure total value added throughout the local economy as a result of a unit increase 
Table 6.4a. Imperial County economy multipliers. ${ }^{2}$

Sector multipliers

\begin{tabular}{|c|c|c|c|c|}
\hline $\begin{array}{l}\text { Column } \\
\text { number }\end{array}$ & $\begin{array}{l}\text { Sector multipliers } \\
\text { Sector name }\end{array}$ & $\begin{array}{l}\text { Output } \\
\text { multiplier }\end{array}$ & $\begin{array}{l}\text { Employment } \\
\text { multiplier }\end{array}$ & $\begin{array}{l}\text { Value } \\
\text { added } \\
\text { multiplier }\end{array}$ \\
\hline 1 & Dairies & 1.51225 & 1.2252 & 1.5494 \\
\hline 2 & Poultry and eggs & 1.79968 & 1.5544 & 2.9848 \\
\hline 3 & $\begin{array}{l}\text { Meat animals and misc. } \\
\text { livestock }\end{array}$ & 2.00638 & 2.1831 & 2.3905 \\
\hline 4 & Cotton & 1.36598 & 1.2612 & 1.6232 \\
\hline 5 & $\begin{array}{l}\text { Food feed grains and grass } \\
\text { seeds }\end{array}$ & 1.36380 & 4.4723 & 1.3719 \\
\hline 6 & Fruits and tree nuts & 1.23597 & 1.1968 & 1.2395 \\
\hline 7 & $\begin{array}{l}\text { Vegetables, sugar beets, and } \\
\text { misc. crops }\end{array}$ & 1.25002 & 1.8597 & 1.2414 \\
\hline 8 & $\begin{array}{l}\text { Greenhouse and nursery } \\
\text { products }\end{array}$ & 1.16118 & 1.2019 & 1.1171 \\
\hline 9 & $\begin{array}{l}\text { Agriculture, forestry, } \\
\text { fishery service }\end{array}$ & 1.01886 & 1.0091 & 1.0160 \\
\hline 10 & $\begin{array}{l}\text { Stone and clay mining and } \\
\text { quarrying }\end{array}$ & 1.19785 & 1.3740 & 1.1969 \\
\hline 11 & $\begin{array}{l}\text { Chemical and fertilizer } \\
\text { mineral mining }\end{array}$ & 1.08607 & 1.2142 & 1.0668 \\
\hline 12 & $\begin{array}{l}\text { New construction, resident, } \\
\text { (nonfarm) }\end{array}$ & 1. & 1.4723 & 1.6033 \\
\hline 13 & New construction, nonresident & 1.27763 & 1.4557 & 1.5539 \\
\hline 14 & $\begin{array}{l}\text { New construction, public } \\
\text { utilities }\end{array}$ & 1.19910 & 1.2693 & 1.3935 \\
\hline 15 & New construction, highways & 1.31991 & 1.4868 & 1.5027 \\
\hline 16 & New construction, all other & 1.21795 & 1.5674 & 1.3365 \\
\hline 17 & $\begin{array}{l}\text { Maintenance and repair } \\
\text { construction, residential }\end{array}$ & 1. 19176 & 1.1495 & 1.2665 \\
\hline 18 & $\begin{array}{l}\text { Maintenance and repair con- } \\
\text { struction, all other }\end{array}$ & 1. 14039 & 1.1996 & 1.1531 \\
\hline 19 & Neat products & 2.27025 & 3.1966 & 3.9571 \\
\hline 20 & $\begin{array}{l}\text { Flour and other grain mill } \\
\text { products }\end{array}$ & 1.46946 & 1.8714 & 1.7688 \\
\hline 21 & $\begin{array}{l}\text { Prepared feeds for animals } \\
\text { and fowls }\end{array}$ & 1.63371 & 1.9161 & 2.9878 \\
\hline
\end{tabular}

${ }^{a}$ Column numbers correspond to those used by Lofting. ${ }^{2}$ 
Table 6.4a. (Continued)

\begin{tabular}{|c|c|c|c|c|}
\hline $\begin{array}{l}\text { Column } \\
\text { number }\end{array}$ & Sector multipliers & $\begin{array}{l}\text { Output } \\
\text { multiplier }\end{array}$ & $\begin{array}{l}\text { Employment } \\
\text { multiplier }\end{array}$ & $\begin{array}{c}\text { Value } \\
\text { added } \\
\text { multiplier }\end{array}$ \\
\hline 22 & Sugar & 2.26589 & 3.0430 & 2.9758 \\
\hline 23 & $\begin{array}{l}\text { Bottled and canned soft } \\
\text { drinks }\end{array}$ & 1.21720 & 1.1670 & 1.2419 \\
\hline 24 & $\begin{array}{l}\text { Animal and marine fats and } \\
\text { oils }\end{array}$ & 1.44362 & 1.6675 & 1.4747 \\
\hline 25 & Manufactured ice & 1.29195 & 1.1781 & 1.3116 \\
\hline 26 & $\begin{array}{l}\text { Apparel made from purchased } \\
\text { materials }\end{array}$ & 1.07409 & 1.0644 & 1.1216 \\
\hline 27 & $\begin{array}{l}\text { Prefabricated wood } \\
\text { structures }\end{array}$ & 1.14459 & 1.1434 & 1.2797 \\
\hline 28 & Wooden containers & 1.09982 & 1.1279 & 1.1745 \\
\hline 29 & Newspapers & 1.12083 & 1.0620 & 1.1232 \\
\hline 30 & Miscellaneous publishing & 1.14008 & 1.1322 & 1.1272 \\
\hline 31 & Commercial printing & 1.09504 & 1.0963 & 1.1115 \\
\hline 32 & Fertilizers & 1.17600 & 1.3927 & 1.3340 \\
\hline 33 & Agricultural chemicals & 1.06508 & 1.1684 & 1.1200 \\
\hline 34 & $\begin{array}{l}\text { Miscellaneous plastics } \\
\text { products }\end{array}$ & 1.09695 & 1.1163 & 1.1423 \\
\hline 35 & Concrete products & 1.19286 & 1.1835 & 1.2003 \\
\hline 36 & Ready-mixed concrete & 1.22910 & 1.3702 & 1.3866 \\
\hline 37 & Gypsum products & 1.13692 & 1.1886 & 1.1481 \\
\hline 38 & Cut stone and stone products & 1.17827 & 1.1751 & 1.1640 \\
\hline 39 & Nonmetallic mineral products & 1.28278 & 1.2466 & 1.3412 \\
\hline 40 & Sheet metalwork & 1.09175 & 1.1090 & 1.1560 \\
\hline 41 & Farm machinery & 1.10598 & 1.1823 & 1.1590 \\
\hline 42 & Machine shop products & 1.12466 & 1.1074 & 1.1186 \\
\hline 43 & Sporting and athletic goods & 1.16711 & 1.1805 & 1.2199 \\
\hline 44 & $\begin{array}{l}\text { Signs and advertising } \\
\text { displays }\end{array}$ & 1.13824 & 1.1503 & 1.1722 \\
\hline 45 & Railroads & 1.08264 & 1.0900 & 1.0717 \\
\hline
\end{tabular}


Table 6.4a. (Continued)

\begin{tabular}{|c|c|c|c|c|}
\hline $\begin{array}{l}\text { Column } \\
\text { number }\end{array}$ & $\begin{array}{l}\text { Sector multipliers } \\
\text { Sector name }\end{array}$ & $\begin{array}{l}\text { Output } \\
\text { multiplier }\end{array}$ & $\begin{array}{l}\text { Employment } \\
\text { multiplier }\end{array}$ & $\begin{array}{l}\text { Value } \\
\text { added } \\
\text { multiplier }\end{array}$ \\
\hline 46 & $\begin{array}{l}\text { Local, suburban, } \\
\text { interurban transportation }\end{array}$ & 1.11682 & 1.0986 & 1.1164 \\
\hline 47 & $\begin{array}{l}\text { Motor freight transportation } \\
\text { and warehousing }\end{array}$ & 1.24710 & 1.2462 & 1.2565 \\
\hline 48 & Transportation services & 1.05974 & 1.0363 & 1.0456 \\
\hline 49 & $\begin{array}{l}\text { Communication except radio } \\
\text { and TV }\end{array}$ & 1.04581 & 1.0404 & 1.0297 \\
\hline 50 & $\begin{array}{l}\text { Radio and television } \\
\text { broadcasting }\end{array}$ & 1.46857 & 1.5086 & 1.3863 \\
\hline 51 & $\begin{array}{l}\text { Electric companies and } \\
\text { systems }\end{array}$ & 1.16884 & 1.6397 & 1.1734 \\
\hline 52 & Water and sanitary services & 1.19601 & 1.4537 & 1.5945 \\
\hline 53 & Wholesale trade & 1.13524 & 1.1103 & 1.1038 \\
\hline 54 & $\begin{array}{l}\text { Retail trade - general } \\
\text { merchandise }\end{array}$ & 1.08 .163 & 1.0395 & 1.0825 \\
\hline 55 & Banking & 1.11077 & 1.1242 & 1.0876 \\
\hline 56 & $\begin{array}{l}\text { Credit agencies other than } \\
\text { banks }\end{array}$ & 1.60238 & 1.3535 & 0.0000 \\
\hline 57 & $\begin{array}{l}\text { Insurance agents, brokers, } \\
\text { and service }\end{array}$ & 1.23351 & 1.2595 & 1.2189 \\
\hline 58 & Owner occupied dwellings & 1.05663 & 0.0000 & 1.0405 \\
\hline 59 & Real estate & 1.18286 & 1.5230 & 1.1395 \\
\hline 60 & $\begin{array}{l}\text { Hotels, rooming houses, } \\
\text { camps, etc }\end{array}$ & 1.17622 & 1.0347 & 1.1289 \\
\hline 61 & Personal services & 1.07235 & 1.0420 & 1.0527 \\
\hline 62 & Barber and beauty shops & 1.00143 & 1.0004 & 1.0012 \\
\hline 63 & $\begin{array}{l}\text { Miscellaneous business } \\
\text { services }\end{array}$ & 1.15393 & 1.1208 & 1.1434 \\
\hline 64 & Miscellaneous services & 1.21328 & 1.1743 & 1.1622 \\
\hline 65 & $\begin{array}{l}\text { Car repair, services, and } \\
\text { garages }\end{array}$ & 1.26466 & 1.4779 & 1.6653 \\
\hline 66 & Motion pictures & 1.80112 & 1.9235 & 1.8605 \\
\hline 67 & $\begin{array}{l}\text { Amusement and recreation } \\
\text { services }\end{array}$ & 1.20222 & 1.0909 & 1.1581 \\
\hline
\end{tabular}


Table 6.4a. (Continued)

Sector multipliers

\begin{tabular}{|c|c|c|c|c|}
\hline $\begin{array}{l}\text { Column } \\
\text { number }\end{array}$ & 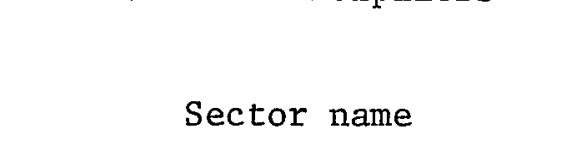 & $\begin{array}{l}\text { Output } \\
\text { multiplier }\end{array}$ & $\begin{array}{l}\text { Employment } \\
\text { multiplier }\end{array}$ & $\begin{array}{c}\text { Value } \\
\text { added } \\
\text { multiplier }\end{array}$ \\
\hline 68 & Doctors and dentists & 1.19603 & 1.2700 & 1.1803 \\
\hline 69 & Health and allied services & 1.25455 & 1.1572 & 1.2729 \\
\hline 70 & Educational services & 1.23307 & 1.1139 & 1.2685 \\
\hline 71 & Nonprofit organization & 1.27940 & 1.1100 & 1.2874 \\
\hline 72 & $\begin{array}{l}\text { Federa1 government } \\
\text { enterprises }\end{array}$ & 1.17700 & 1.1357 & 1.1255 \\
\hline 73 & $\begin{array}{l}\text { State and local government } \\
\text { enterprises }\end{array}$ & 1.14652 & 1.1016 & 1.1242 \\
\hline 74 & $\begin{array}{l}\text { Business trave1, } \\
\text { entertainment, gifts }\end{array}$ & 1.24467 & 0.0000 & 0.0000 \\
\hline 75 & Office supplies & 1.90715 & 0.0000 & 0.0000 \\
\hline 76 & $\begin{array}{l}\text { Scrap, used, secondhand } \\
\text { goods }\end{array}$ & 1.05418 & 0.0000 & 0.0000 \\
\hline
\end{tabular}

in any single sector. Sectors with high multiplier values generate the greatest benefit for the economy as a whole when their output is increased. Thus from the ranking in Table $6.4 \mathrm{~b}$, it is seen that increases in feed lot operations and meat production generate considerable total activity, while an increase in barber shops will have no real effect on the economy. Because the Imperial County economy has traditionally been centered around agricultural products, those sectors associated with agriculture tend to have a greater effect upon other sectors in the economy and, therefore, tend to have higher multipliers.

\subsection{AGRICULTURAL SECTOR ANALYSIS}

The value and amount of planted acreage of the major Imperial Valley crops are well-documented by the county and the state. Data from recent years are summarized in Table 6.5. Recent increases in total planted acreage can be attributed primarily to an increase in double planting on many fields. The distribution and characteristics of crops within the valley are described in Section 4 (Biological Resources) of this report, as well 
Table 6.4b. Ranked sector multipliers: output multiplier.

\begin{tabular}{|c|c|c|c|}
\hline $\begin{array}{l}\text { Column } \\
\text { number }\end{array}$ & Sector name & $\begin{array}{l}\text { Output } \\
\text { multiplier }\end{array}$ & $\begin{array}{c}\text { Output } \\
\text { multiplior } \\
\text { ranl: }\end{array}$ \\
\hline 19 & Meat products & 2.2702498 & 1 \\
\hline 22 & Sugar & 2.2658885 & 2 \\
\hline 3 & Meat animals and misc. livestock & 2.0063831 & 3 \\
\hline 66 & Motion pictures & 1.8011162 & 4 \\
\hline 2 & Poultry and eggs & 1.7996829 & 5 \\
\hline 21 & Prepared feeds for animals and fowls & 1.6337148 & 6 \\
\hline 56 & Credit agencies other than banks & 1.6023821 & 7 \\
\hline 1 & Dairies & 1.5122523 & 8 \\
\hline 20 & Flour and other grain mill products & 1.4694599 & 9 \\
\hline 50 & Radio and television broadcasting & 1.4685672 & 10 \\
\hline 24 & Animal and marine fats and oils & 1.4436233 & 11 \\
\hline 4 & Cotton & 1.3659842 & 12 \\
\hline 5 & Food feed grains and grass seeds & 1.3638000 & 13 \\
\hline 15 & New construction, highways & 1.3199098 & 14 \\
\hline 12 & New construction, resident, (nonfarm) & 1.3011469 & 15 \\
\hline 25 & Manufactured ice & 1.2919542 & 16 \\
\hline 39 & Nonmetallic mineral products, nec & 1.2827828 & 17 \\
\hline 71 & Nonprofit organization & 1.2794011 & 18 \\
\hline 13 & New construction, nonresident & 1.2776295 & 19 \\
\hline 65 & Car repair, services, and garages & 1.2646639 & 20 \\
\hline 69 & Health and allied services, nec. & 1.2545470 & 21 \\
\hline 7 & Vegetables, sugar beets, and misc. crops & 1.2500204 & 22 \\
\hline 47 & Notor freight transportation and warehousing & $\mathrm{g} 1.2471033$ & 23 \\
\hline 74 & Business travel, entertainment, gifts & 1.2446683 & 24 \\
\hline 6 & Fruits and tree nuts & 1.2359728 & 25 \\
\hline 57 & Insurance agents, brokers, and service & 1.2335088 & 26 \\
\hline 70 & Educational services & 1.2330731 & 27 \\
\hline 36 & Ready-mixed concrete & 1.2291017 & 28 \\
\hline 16 & INew construction, all other & 1.2179537 & 29 \\
\hline
\end{tabular}




\begin{tabular}{|c|c|c|c|}
\hline $\begin{array}{l}\text { Column } \\
\text { number }\end{array}$ & Sector name & $\begin{array}{l}\text { Output } \\
\text { multiplier }\end{array}$ & $\begin{array}{c}\text { Output } \\
\text { multiplier } \\
\text { rank }\end{array}$ \\
\hline 23 & Bottled and canned soft drinks & 1.2172030 & 30 \\
\hline 64 & Miscellaneous services & 1.2132768 & 3.1 \\
\hline 67 & Amusement and recreation services & 1.2022155 & 32 \\
\hline 14 & New construction, public utilities & 1.1991023 & 33 \\
\hline 10 & Stone and clay mining and quarrying & 1.1978533 & 34 \\
\hline 68 & Doctors and dentists & 1.1960322 & 35 \\
\hline 52 & Water and sanitary services & 1.1960103 & 36 \\
\hline 35 & Concrete products & 1.1928626 & 37 \\
\hline 17 & $\begin{array}{l}\text { Maintenance and repair construction, } \\
\text { residential }\end{array}$ & 1.1917602 & 38 \\
\hline 59 & Real estate & 1.1828574 & 39 \\
\hline 38 & Cut stone and stone products & 1.1782667 & 40 \\
\hline 72 & Federal government enterprises & 1.1770037 & 41 \\
\hline 60 & Hotels, rooming houses, camps, etc & 1.1762201 & 42 \\
\hline 32 & Fertilizers & 1.1760041 & 43 \\
\hline 51 & Electric companies and systems & 1.1688362 & 44 \\
\hline 43 & Sporting and athletic goods & 1.1671086 & 45 \\
\hline 8 & Greenhouse and nursery products & 1.1611817 & 46 \\
\hline 63 & Miscellaneous business services & 1.1539346 & 47 \\
\hline 73 & State and local government enterprises & 1.1465184 & 48 \\
\hline 27 & Prefabricated wood structures & 1.1445854 & 49 \\
\hline 18 & $\begin{array}{l}\text { Maintenance and repair construction, } \\
\text { a11 other }\end{array}$ & 1.1403895 & 50 \\
\hline 30 & Miscellaneous publishing & 1.1400810 & 51 \\
\hline 44 & Signs and advertising displays & 1.1382421 & 52 \\
\hline 37 & Gypsum products & 1.1369190 & 53 \\
\hline 53 & Wholesale trade & 1.1362421 & 54 \\
\hline 42 & Machine shop products & 1.1246599 & 55 \\
\hline 29 & Newspapers & 1.1208275 & 56 \\
\hline 46 & Local, suburban, interurban trans. & 1.1168203 & 57 \\
\hline
\end{tabular}




\begin{tabular}{|c|c|c|c|}
\hline $\begin{array}{l}\text { Column } \\
\text { number }\end{array}$ & Sector name & $\begin{array}{c}\text { Output } \\
\text { multiplier }\end{array}$ & $\begin{array}{c}\text { Output } \\
\text { multiplier } \\
\text { rant }\end{array}$ \\
\hline 55 & Banking & 1.1107723 & 58 \\
\hline 41 & Farm machinery & 1.1059827 & 59 \\
\hline 28 & Wooden containers & 1.0998196 & 60 \\
\hline 75 & Office supplies & 1.0971536 & 61 \\
\hline 34 & Miscellaneous plastics products & 1.0969475 & 62 \\
\hline 31 & Commercial printing & 1.0950372 & 63 \\
\hline 40 & Sheet metalwork & 1.0917474 & 64 \\
\hline 11 & Chemical and fertilizer mineral mining & 1.0860722 & 65 \\
\hline 45 & Railroads & 1.0826401 & 66 \\
\hline 54 & Retail trade - general merchandise & 1.0816292 & 67 \\
\hline 26 & Apparel made from purchased materials & 1.0740925 & 68 \\
\hline 61 & Personal services & 1.0723509 & 69 \\
\hline 33 & Agricultural chemicals & 1.0650765 & 70 \\
\hline 48 & Transportation services & 1.0597393 & 71 \\
\hline 58 & Owner occupied dwellings & 1.0566330 & 72 \\
\hline 76 & Scrap, used, secondhand goods & 1.0541842 & 73 \\
\hline 49 & Communication except radio and TV & 1.0458066 & 74 \\
\hline 9 & Agric., forestry, and fishery services & 1.0188616 & 75 \\
\hline 62 & Barber and beauty shops & 1.0014331 & 76 \\
\hline
\end{tabular}

as data on temporal cycling of the planting, growing, and harvesting seasons of most major crops.

Long-term trends in the agricultural cropping patterns are very difficult to predict and result from a series of external economic, natural, and social factors, including national market demands and preferences, weather patterns, international trade, existing cropping patterns, etc. There is a significant trend toward mechanization for many crops. This trend is expected to continue as labor prices, currently above $\$ 2.50$ per hour throughout the valley, ${ }^{\dagger}$ climb above the capital

\footnotetext{
The teamster union contract requires a minimum of $\$ 2.95$ for preharvest and $\$ 3.03$ for harvest period. 10
} 
Table 6.4c. Ranked sector multipliers: employment multiplier.

\begin{tabular}{|c|c|c|c|}
\hline $\begin{array}{l}\text { Column } \\
\text { number }\end{array}$ & Sector name & $\begin{array}{l}\text { Employment } \\
\text { multiplier }\end{array}$ & $\begin{array}{c}\text { Employment } \\
\text { multiplier } \\
\text { rank }\end{array}$ \\
\hline 5 & Food feed grains and grass seeds & 4.47228 & 1 \\
\hline 19 & Meat products & 3.19663 & 2 \\
\hline 22 & Sugar & 3.04300 & 3 \\
\hline 3 & Meat animals and misc. livestock & 2.18310 & 4 \\
\hline 66 & Motion pictures & 1.92354 & 5 \\
\hline 21 & Prepared feeds for animals and fowls & 1.91608 & 6 \\
\hline 20 & Flour and other grain mil1 products & 1.87142 & 7 \\
\hline 7 & Vegetables, sugar beets, and misc. crops & 1.85970 & 8 \\
\hline 24 & Animal and marine fats and oils & 1.66747 & 9 \\
\hline 51 & Electric companies and systems & 1.63975 & 10 \\
\hline 16 & New construction, all other & 1.56739 & 11 \\
\hline 2 & Poultry and eggs & 1.55443 & 12 \\
\hline 59 & Real estate & 1.52296 & 13 \\
\hline 50 & Radio and television broadcasting & 1.50858 & 14 \\
\hline 15 & New construction, highways & 1.48682 & 15 \\
\hline 65 & Car repair, services, and garages & 1.47791 & 16 \\
\hline 12 & New construction, resident, (nonfarm) & 1.47227 & 17 \\
\hline 13 & New construction, nonresident & 1.45575 & 18 \\
\hline 52 & Water and sanitary services & 1.45371 & 19 \\
\hline 32 & Fertilizers & 1.39274 & 20 \\
\hline 10 & Stone and clay mining and quarrying & 1.37400 & 21 \\
\hline 36 & Ready-mixed concrete & 1.37019 & 22 \\
\hline 56 & Credit agencies other than banks & 1.35345 & 23 \\
\hline 68 & Doctors and dentists & 1.27003 & 24 \\
\hline 14 & New construction, public utilities & 1.26925 & 25 \\
\hline 4 & Cotton & 1.26120 & 26 \\
\hline 57 & Insurance agents, brokers, and service & 1.25949 & 27 \\
\hline 39 & Nonmetallic mineral products & 1.24664 & 28 \\
\hline 47 & Motor freight transp. and warehousing & 1.24620 & 29 \\
\hline
\end{tabular}


Column number:
Employment

Employment multiplier multiplier

rank:

\begin{tabular}{|c|c|c|}
\hline 1 & Dairies & 1.22517 \\
\hline 11 & Chemical and fertilizer mineral mining & 1.21419 \\
\hline 8 & Greenhouse and nursery products & 1.20193 \\
\hline 18 & $\begin{array}{l}\text { Maintenance and repair construction, } \\
\text { all other }\end{array}$ & 1.19957 \\
\hline 6 & Fruits and tree nuts & 1.19681 \\
\hline 37 & Gypsum products & 1.18857 \\
\hline 35 & Concrete products & 1.18348 \\
\hline 41 & Farm machinery & 1.18231 \\
\hline 43 & Sporting and athletic goods & 1.18052 \\
\hline 25 & Manufactured ice & 1.17815 \\
\hline 38 & Cut stone and stone products & 1.17508 \\
\hline 64 & Miscellaneous services & 1.17430 \\
\hline 33 & Agricultural chemicals & 1.16845 \\
\hline 23 & Bottled and canned soft drinks & 1.16700 \\
\hline 69 & Health and allied services & 1.15717 \\
\hline 44 & Signs and advertising displays & 1.15033 \\
\hline 17 & $\begin{array}{l}\text { Maintenance and repair construction, } \\
\text { residential }\end{array}$ & 1.14945 \\
\hline 27 & Prefabricated wood structures & 1.14335 \\
\hline 72 & Federal government enterprises & 1.13570 \\
\hline 30 & Miscellaneous publishing & 1.13219 \\
\hline 28 & Wooden containers & 1.12765 \\
\hline 55 & Banking & 1.12420 \\
\hline 63 & Miscellaneous business services & 1.12076 \\
\hline 34 & Miscellaneous plastics products & 1.11626 \\
\hline 70 & Educational services & 1.11393 \\
\hline 53 & Wholesale trade & 1.11033 \\
\hline 71 & Nonprofit organization & 1.11005 \\
\hline
\end{tabular}




\begin{tabular}{|c|c|c|c|}
\hline $\begin{array}{l}\text { Column } \\
\text { number }\end{array}$ & Sector name & $\begin{array}{l}\text { Employment } \\
\text { multiplier }\end{array}$ & $\begin{array}{c}\text { Employment } \\
\text { multiplier } \\
\text { rank }\end{array}$ \\
\hline 40 & Sheet metalwork & 1.10900 & 57 \\
\hline 42 & Machine shop products & 1.10739 & 58 \\
\hline 73 & State and local government enterprises & 1.10158 & 59 \\
\hline 46 & Local, surburban, interurban transportation & 1.09860 & 60 \\
\hline 31 & Commercial printing & 1.09625 & 61 \\
\hline 67 & Amusement and recreation services & 1.09090 & 62 \\
\hline 45 & Railroads & 1.09000 & 63 \\
\hline 26 & Apparel made from purchased materials & 1.06439 & 64 \\
\hline 29 & Newspapers & 1.06203 & 65 \\
\hline 61 & Personal services & 1.04205 & 66 \\
\hline 49 & Communication except radio and TV & 1.04038 & 67 \\
\hline 54 & Retail trade - general merchandise & 1.03949 & 68 \\
\hline 48 & Transportation services & 1.03633 & 69 \\
\hline 60 & Hotels, rooming houses, camps, etc & 1.03473 & 70 \\
\hline 9 & $\begin{array}{l}\text { Agricultural, forestry, and fishery } \\
\text { services }\end{array}$ & 1.00907 & 71 \\
\hline 62 & Barber and beauty shops & 1.00043 & 72 \\
\hline 58 & Owner occupied dwellings & 0.00000 & 73 \\
\hline 74 & Business travel, entertainment, gifts & 0.00000 & 74 \\
\hline 75 & Office supplies & 0.00000 & 75 \\
\hline 76 & Scrap, used, secondhand goods & 0.00000 & 76 \\
\hline
\end{tabular}

and operational costs of mechanized systems. An important factor in either shifts to mechanization or shifts to alternate crops is the cost of production for that crop. These costs are compared in Table 6.6 for selected crops.
The data in Tables 6.5 and 6.6 reveal a considerable amount of information about Imperial Valley agriculturial economics. As seen in Table 6.5, field crops with a total value of $\$ 284,242,000$ became the first crop group to break 
Table 6.4d. Ranked sector multipliers: value added multiplier.

\begin{tabular}{|c|c|c|c|}
\hline $\begin{array}{l}\text { Column } \\
\text { number }\end{array}$ & Sector name & $\begin{array}{c}\text { Value } \\
\text { added } \\
\text { multiplier }\end{array}$ & $\begin{array}{c}\text { Valuc } \\
\text { added } \\
\text { multiplier } \\
\text { ranl: }\end{array}$ \\
\hline 19 & Meat products & 3.95713 & 1 \\
\hline 21 & Prepared feeds for animals and fowls & 2.98783 & 2 \\
\hline 2 & Poultry and eggs & 2.98483 & 3 \\
\hline 22 & Sugar & 2.97584 & 4 \\
\hline 3 & Meat animals and misc. livestock & 2.39046 & 5 \\
\hline 66 & Motion pictures & 1.86049 & 6 \\
\hline 20 & Flour and other grain mill products & 1.76881 & 7 \\
\hline 65 & Car repair, services, and garages & 1.66532 & 8 \\
\hline 4 & Cotton & 1.62320 & 9 \\
\hline 12 & New construction, residential, (nonfarm & 1.60329 & 10 \\
\hline 52 & Water and sanitary services & 1.59451 & 11 \\
\hline 13 & New construction, nonresidential & 1.55386 & 12 \\
\hline 1 & Dairies & 1.54944 & 13 \\
\hline 15 & New construction, highways & 1.50274 & 14 \\
\hline 24 & Animal and marine fats and oils & 1.47469 & 15 \\
\hline 14 & New construction, public utilities & 1.39351 & 16 \\
\hline 36 & Ready-mixed concrete & 1.38664 & 17 \\
\hline 50 & Radio and television broadcasting & 1.38631 & 18 \\
\hline 5 & Food feed grains and grass seeds & 1.37191 & 19 \\
\hline 39 & Nonmetallic mineral products & 1.34116 & 20 \\
\hline 16 & New construction, all other & 1.33652 & 21 \\
\hline 32 & Fertilizers & 1.33399 & 22 \\
\hline 25 & Manufactured ice & 1.31159 & 23 \\
\hline 71 & Nonprofit organization & 1.28739 & 24 \\
\hline 27 & Prefabricated wood structures & 1.27968 & 25 \\
\hline 69 & Health and allied services & 1.27285 & 26 \\
\hline 70 & Educational services & 1.26851 & 27 \\
\hline 17 & $\begin{array}{l}\text { Maintenance repair and construction, } \\
\text { residential }\end{array}$ & 1.26654 & 28 \\
\hline
\end{tabular}


Column

number
Sector name
Value

Value

added

multiplier added multiplier rank

47 Motor freight transportation and warehousing

1.25653

29

23 Bottled and canned soft drinks

1.24185

30

7 Vegetables, sugar beets, and misc. crops

1.24137

31

6 Fruits and tree nuts

1.23953

32

43 Sporting and athletic goods

1.21990

33

57 Insurance agents, brokers, and service

1.21895

34

35 Concrete products

1.20026

35

10 Stone and clay mining and quarxying

1.19689

36

68 Doctors and dentists

1.18026

37

28 Wooden containers

1.17446

38

51 Electric companies and systems

1.17335

39

44 Signs and advertising displays

1.17225

40

38 Cut stone and stone products

1.16395

41

64 Miscellaneous services

1.16218

42

41 Farm machinery

1.15904

43

67 Amusement and recreation services

1.15809

44

40 Sheet metalwork

1.15599

18 Maintenance and repair construction, all other

1.15313

46

37 Gypsum products

1.14805

47

63 Miscellaneous business services

1.14338

48

34 Miscellaneous plastics products

1.14229

49

59 Real estate

1.13947

50

60 Hotels, rooming houses, camps, etc

1.12889

51

30 Miscellaneous publishing

1.12721

52

72 Federal government enterprises

1.12549

53

73 State and local government enterprises

1.12421

54

29 Newspapers

1. 12321

55 
Table 6.4d. (Concluded)

\begin{tabular}{|c|c|c|c|}
\hline $\begin{array}{l}\text { Column } \\
\text { number }\end{array}$ & Sector name & $\begin{array}{c}\text { Value } \\
\text { added } \\
\text { multiplier }\end{array}$ & $\begin{array}{c}\text { value } \\
\text { added } \\
\text { multiplier } \\
\text { ranl }\end{array}$ \\
\hline 26 & Apparel made from purchased materials & 1.12161 & 56 \\
\hline 33 & Agricultural chemicals & 1.12003 & 57 \\
\hline 42 & Machine shop products & 1.11855 & 58 \\
\hline 8 & Greenhouse and nursery products & 1.11706 & 59 \\
\hline 46 & Local, suburban, interurban transportation & 1.11638 & 60 \\
\hline 31 & Commercial printing & 1.11152 & 61 \\
\hline 53 & Wholesale trade & 1.10384 & 62 \\
\hline 55 & Banking & 1.08757 & 63 \\
\hline 45 & Railroads & 1.07165 & 64 \\
\hline 11 & Chemical and fertilizer mineral mining & 1.06681 & 65 \\
\hline 54 & Retail trade - general merchandise & 1.06248 & 66 \\
\hline 61 & Personal services & 1.05273 & 67 \\
\hline 48 & Transportation services & 1.04565 & 68 \\
\hline 58 & Owner occupied dwellings & 1.04054 & 69 \\
\hline 49 & Communication except radio and TV & 1.02971 & 70 \\
\hline 9 & Agriculture, forestry, and fishery services & 1.01603 & 71 \\
\hline 62 & Barber and beauty shops & 1.00118 & 72 \\
\hline 56 & Credit agencies other than banks & 0.00000 & 73 \\
\hline 74 & Business travel, entertainment, gifts & 0.00000 & 74 \\
\hline 75 & Office supplies & 0.00000 & 75 \\
\hline 76 & Scrap, used, secondhand goods & 0.00000 & 76 \\
\hline
\end{tabular}


Table 6.5. Acreage and value of major Imperial Valley agriculture for selected years.

\begin{tabular}{|c|c|c|c|c|c|c|c|}
\hline \multirow[b]{2}{*}{ Crop } & \multicolumn{2}{|c|}{$1968^{4,5}$} & \multicolumn{2}{|c|}{$1969^{4,5,6}$} & \multicolumn{2}{|c|}{$1973^{7,8}$} & \multirow[b]{2}{*}{ Acrea } \\
\hline & Acreage & Value & Acreage & Value & Acreage & Value & \\
\hline Field crops & & $\$ 92,220,000$ & & $\$ 90,490,000$ & 473,422 & $\$ 176,311,000$ & 475,6 \\
\hline Alfalfa & 136,000 & & 140,000 & $27,608,000$ & 124,567 & $53,424,000$ & 125,6 \\
\hline Alfalfa seed & 15,000 & $1,512,000$ & 7,300 & 648,000 & 1,660 & & 2,3 \\
\hline Alicia grass & & & & & 2,722 & & 2,7 \\
\hline Barley & 99,000 & & 74,000 & $7,696,000$ & 17,433 & $2,520,000$ & 5,3 \\
\hline Bermuda grass & & & & & 1,968 & & 2,4 \\
\hline Bermuda grass (seed) & & & & & 964 & & 9 \\
\hline Cotton & 38,190 & & 44,000 & $17,499,000$ & 36,857 & $30,160,000$ & 78,8 \\
\hline Flax & 2,000 & & 1,800 & 246,000 & 80 & & \\
\hline Oats & & & & & 1,245 & & 1,0 \\
\hline Rape & & & & & - & & \\
\hline Rye grass & & & & & 27,456 & $3,770,000$ & 18,8 \\
\hline Rye .grass (seed) & 620 & 13,000 & 80 & 2,600 & 509 & & 2 \\
\hline Safflower & 450 & & - & & 16 & & - \\
\hline Sesbania & & & & & - & & - \\
\hline Sesbania (seed) & 230 & 17,000 & 225 & 27,400 & 79 & - & - \\
\hline Sorghum grain & 75,000 & & 60,000 & $7,350,000$ & 39,389 & $8,280,000$ & 31,6 \\
\hline Sorghum silage & 1,000 & & 2,000 & 220,000 & 1,032 & 138,000 & 4 \\
\hline Sudan grass & & & & & 13,224 & & 14,4 \\
\hline Sugar beets & 59,200 & & 76,000 & $22,876,000$ & 69,812 & $31,936,000$ & 69,1 \\
\hline Wheat & 1,000 & & 10,000 & $1,300,000$ & 94,407 & $26,000,000$ & 101,4 \\
\hline Pasture (genera1) & 240,000 & & 243,000 & $3,062,000$ & 195,000 & $4,086,000$ & 192,0 \\
\hline
\end{tabular}


Table 6.5. (Continued)

\begin{tabular}{|c|c|c|c|c|c|c|c|}
\hline \multirow[b]{2}{*}{ Crop } & \multicolumn{2}{|c|}{1968} & \multicolumn{2}{|c|}{1969} & \multicolumn{2}{|c|}{1973} & \multirow[b]{2}{*}{ Acre } \\
\hline & Acreage & Value & Acreage & Value & Acreage & Value & \\
\hline Vegetable crops & 64,170 & $\$ 66,231,000$ & 64,625 & $\$ 66,522,000$ & 74,870 & $\$ 119,102,000$ & 84, \\
\hline Asparagus & 2,200 & $1,963,000$ & 2,400 & $2,523,000$ & 4,500 & $5,832,000$ & 4, \\
\hline Cabbage & 2,710 & $1,723,000$ & 1,300 & 782,000 & 470 & 619,000 & 1, \\
\hline Carrots & 4,900 & $5,145,000$ & 4,200 & $4,150,000$ & 4,500 & $7,092,000$ & 5, \\
\hline Cucumbers & 380 & 560,000 & 390 & 277,000 & 520 & 527,000 & \\
\hline Let tuce & 41,500 & $34,109,000$ & 44,000 & $33,683,000$ & 42,000 & $73,200,000$ & 49, \\
\hline Melons (other) & 930 & $1,136,000$ & 720 & $1,100,000$ & 760 & $1,515,000$ & \\
\hline Onions & 1,700 & $2,617,000$ & 2,300 & $1,495,000$ & 1,500 & $6,304,000$ & 1 \\
\hline Onions (dehydrated) & 1,070 & 444,000 & 1,240 & 560,000 & 1,680 & 740,000 & 3 \\
\hline Syuasin & 750 & $6 ? ? .0 \mathrm{nn}$ & 340 & 320,000 & 940 & 749,000 & \\
\hline Tomatoes & 1,940 & $2,943,000$ & 2,200 & $3,414,000$ & 2,000 & $4,388,000$ & 3 , \\
\hline Tomatoes (caunery) & 280 & 147,000 & - & - & - & & - \\
\hline Wat ermelons & 4,410 & $1,588,000$ & 4,100 & $1,613,000$ & 4,000 & $2,935,000$ & 2 \\
\hline Mixed vegetables & 1,400 & $1,365,000$ & 1,435 & $1,605,000$ & 2,500 & $2,697,000$ & 2 \\
\hline Cantaloupes & - & - & - & - & 9,500 & $12,504,000$ & $8:$ \\
\hline Garlic & & & & & - & - & \\
\hline
\end{tabular}


Table 6.5. (Continued)

\begin{tabular}{|c|c|c|c|c|c|c|c|}
\hline \multirow[b]{2}{*}{ Crop } & \multicolumn{2}{|c|}{1968} & \multicolumn{2}{|c|}{1969} & \multicolumn{2}{|c|}{1973} & \multirow[b]{2}{*}{ Ac } \\
\hline & Acreage & Value & Acreage & Value & Acreage & Value & \\
\hline Fruits and nuts & 1,680 & $\$ 785,000$ & 2,030 & $\$ 988,000$ & 2,916 & $\$ 2,235,000$ & : \\
\hline Dates & 70 & 91,000 & 35 & 119,000 & 140 & 212,000 & \\
\hline Grapefruit & 400 & 266,000 & 300 & 156,000 & 400 & 168,000 & \\
\hline Lemons & 130 & 39,000 & 230 & 230,000 & 620 & 654,000 & \\
\hline Oranges (Valencia) & 750 & 251,000 & 750 & 311,000 & 920 & 406,000 & \\
\hline Tangerines & 330 & 123,000 & 400 & 161,000 & 736 & 486,000 & \\
\hline Mixed fruit & & 15,000 & & 11,000 & 100 & 18,000 & \\
\hline Citrus by-product & & & & & & 291,000 & \\
\hline Apiary & & $\$ 273,500$ & & $\$ 338,000$ & & $\$ 1,478,000$ & \\
\hline Honey & 36,000 & 106,000 & 31,000 & 164,000 & 39,000 & $1,196,000$ & 4: \\
\hline Wax & 36,000 & 10,500 & 31,000 & 15,000 & 39,000 & 32,000 & 4: \\
\hline \multirow[t]{2}{*}{ Pollination } & 32,000 & 157,000 & 32,700 & 159,000 & 29,000 & 250,000 & $2 !$ \\
\hline & \multicolumn{2}{|c|}{1968} & \multicolumn{2}{|c|}{1969} & \multicolumn{2}{|c|}{1973} & \\
\hline Crop & Head & Value & Head & Value & Head & Value & $\mathrm{H} \epsilon$ \\
\hline Livestock & & $\$ 72,892,000$ & & $\$ 88,393,000$ & & $\$ 190,644,000$ & \\
\hline Cattle & 560,000 & $68,264,000$ & 578,000 & $84,623,000$ & 798,000 & $186,461,000$ & $72 C$ \\
\hline Sheep & 160,000 & $2,362,000$ & 100,000 & $1,641,000$ & 160,000 & $2,873,000$ & $16 c$ \\
\hline Wool & 167,000 & 366,000 & 110,000 & 219,000 & 180,000 & 689,000 & 162 \\
\hline Milk & & $1,799,000$ & & $1,808,000$ & & 599,000 & \\
\hline $\begin{array}{l}\text { Miscellaneous } \\
\text { livestock }\end{array}$ & & & & & & 22,000 & \\
\hline Hogs & 2,000 & 84,000 & 1,500 & 72,000 & & & \\
\hline Chicken (hens) & 2,500 & 600 & 2,500 & 1,000 & & & \\
\hline Eggs & & 26,300 & & 29,000 & & & \\
\hline
\end{tabular}


Table 6.6. Approximate (1975 dollars) costs of major Imperial Valley crops per acre per

\begin{tabular}{|c|c|c|c|c|c|}
\hline Crop & Fixed costs & Land preparation & $\begin{array}{c}\text { Planting } \\
\text { (Crop } \\
\text { establishment) }\end{array}$ & $\begin{array}{l}\text { Cu1tural } \\
\text { costs }\end{array}$ & $\mathrm{Ha}$ \\
\hline Alfalfa & $71.74^{\mathrm{a}}$ & $\$ 31.30^{\mathrm{a}}$ & $\$ 18.17^{\mathrm{a}}$ & $\$ 32.23^{a}$ & 4: \\
\hline Cereal crops & 130.30 & 50.50 & 17.00 & 34.55 & 3: \\
\hline Cotton & 188.65 & 65.75 & 26.95 & 209.65 & 101 \\
\hline Rye grass & 107.29 & 38.50 & 11.70 & 51.75 & 1 \\
\hline Sorghum (grain) & 131.72 & 55.00 & 11.90 & 40.25 & 3: \\
\hline Sugar beets & 200.29 & 103.65 & 27.50 & 207.45 & 91 \\
\hline Asparagus & $236.89-278.26^{b}$ & $120.10^{\mathrm{a}}$ & 88.25 & $\begin{array}{l}365.75 \mathrm{c}^{-} \\
243.00^{\mathrm{c}}\end{array}$ & $\begin{array}{r}401 \\
1,351\end{array}$ \\
\hline Cabbage & 237.05 & 99.38 & 35.50 & 440.50 & 1,00 \\
\hline Carrots & 215.49 & 131.88 & 19.50 & 244.38 & 16 \\
\hline Lettuce & 236.72 & 99.38 & 39.50 & 433.75 & 851 \\
\hline Tomatoes & 244.56 & 109.30 & 26.25 & 502.45 & 1,65 \\
\hline
\end{tabular}

One-third of total costs for 3-yr stand of alfalfa.

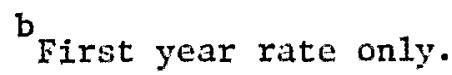

c Last figures for established field. 
the lead status of livestock as the most valuable activity in 1974. Over the 1969 to 1974 period, field crops, especially alfalfa, have been increasing in value and in planted acreage much faster than any other agricultural group and, as noted, much faster than livestock. In fact, of the five major groups listed in Table 6.5 field crops is the only one that did not decline in value in 1974 even though planted acreage for field crops rose only $0.5 \%$, while vegetable crops rose $15 \%$ in planted acreage and fruits and nuts rose 10\%. Over the 1972 to 1974 period there has been a steady decline in the number of cattle ralsed in Imperial Valley and, associated with this decline, a steady decline in the unit value of cattle after feed lot fattening. These trends seem to indicate that farmers are shifting toward field crops over other uses. However, when crop value per acre is calculated from Table 6.5, we see that field crops and fruits and nuts yielded identical gross returns of $\$ 662.50$ per acre, while vegetable crops yielded almost twice that amount ( $\$ 1,222.80$ per acre). This high return per acre for vegetables should have driven many more acres of production toward vegetables than it has. The reason for a continued growth of field crops relative to vegetables, rather than the reverse, can be found in Table 6.6, which gives capital costs associated with each major crop. Vegetable crops are three to nine times more capital intensive than field crops. Thus the increased dollar yield per acre of vegetable crops does not represent a significantly higher rate of return on initial investment.

\subsection{IMPERIAL COUNTY EMPLOYMENT AND LABOR FORCE}

Nonagricultural Employment

Employment data for Imperial County are compiled annually according to nonagricultural employment categories by the State Office of Employment Data and Research and are presented for 1971 to 1975 in Table 6.7. There is a general similarity between the employment category headings in Table 6.7 and the major economic sector divisions in section 6.1 (Table 6.1). Over the 6-yr period covered by Table 6.7 , county manufacturing employment rose $26.7 \%$, about the same as most other sectors. However essentially all of this growth came under Other manufacturing ( $81.8 \%$ growth) and is associated primarily with the new industrial activities listed in Table 6.3. Food manufacturing employment rose much less than did other sectors, while stone, clay, and glass manufacturing was the only sector 
Table 6.7. Imperial County nonagricultural employment. ${ }^{12,13}$

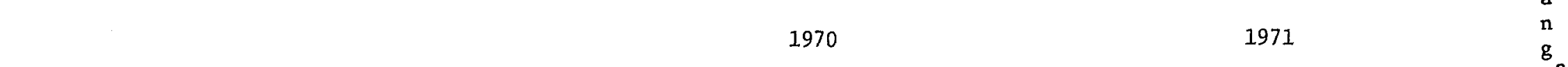

Employment category

Mar. Jun. Sep.

\begin{tabular}{|c|c|c|c|c|c|c|c|c|c|}
\hline Civilian labor force & 26,550 & 26,800 & 22,900 & 27,500 & 29,500 & 29,450 & 24,900 & 29,050 & +7.4 \\
\hline $\begin{array}{l}\text { Unemployment } \\
\text { Unemployment rate, seasonal adjustment } \\
\text { Unemployment rate }\end{array}$ & $\begin{array}{r}2,700 \\
9.1 \\
10.2\end{array}$ & $\begin{array}{r}2,150 \\
8.7 \\
8.0\end{array}$ & $\begin{array}{r}1,800 \\
8.6 \\
7.9\end{array}$ & $\begin{array}{r}3,300 \\
10.8 \\
12.0\end{array}$ & $\begin{array}{r}3,900 \\
11.9 \\
13.2\end{array}$ & $\begin{array}{r}3,200 \\
11.9 \\
10.9\end{array}$ & $\begin{array}{r}2,45.0 \\
10.6 \\
9.8\end{array}$ & $\begin{array}{r}3,700 \\
11.4 \\
12.7\end{array}$ & $\begin{array}{l}+31.3 \\
+22.6 \\
+10.7\end{array}$ \\
\hline $\begin{array}{l}\text { Total civilian employment } \\
\text { Self employed } \\
\text { Domestics }\end{array}$ & $\begin{array}{r}23,850 \\
2,400 \\
350\end{array}$ & $\begin{array}{r}24,650 \\
2,400 \\
350\end{array}$ & $\begin{array}{r}21,100 \\
2,400 \\
350\end{array}$ & $\begin{array}{r}24,200 \\
2,400 \\
350\end{array}$ & $\begin{array}{r}25,600 \\
2,450 \\
350\end{array}$ & $\begin{array}{r}26,250 \\
2,450 \\
350\end{array}$ & $\begin{array}{r}22,450 \\
2,450 \\
350\end{array}$ & $\begin{array}{r}25,350 \\
2,450 \\
350\end{array}$ & $\begin{array}{r}+4.9 \\
+2.1 \\
0\end{array}$ \\
\hline
\end{tabular}

Job based

Nonagricultural wage and salary Manufacturing

Food and kindred

Stone, clay, and glass

Other manufacturing

\section{Nonmanufacturing}

Construction

Transportation, communications, utilities

Trade

Wholesale

Retail

Finances, insurance, and real estate

Services

Government

Federal

State and local

18,000
1,450
450
400
600
16,550
500
1,100
5,800
1,400
4,400
500
2,400
6,250
950
5,300

19,250
1,750
750
400
600
17,500
600
1,100
6,400
2,050
4,350
500
2,350
6,550
950
5,600
17,800
1,450
550
400
500
16,350
550
1,050
5,400
1,050
4,350
500
2,300
6,550
1,000
5,550

18,350

1,350
1,350

40

17,000
550

1,100

6,000

1,250

4,750

2300

6,550

1,000

5,550

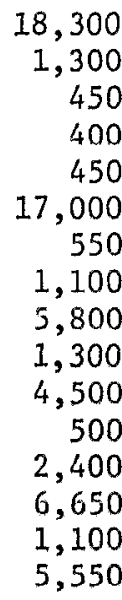

5,550

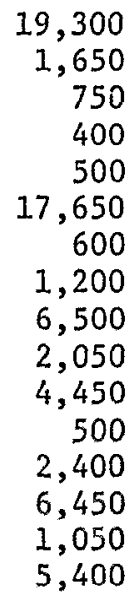
18,050
1,450
550
400
500
16,600
500
1,100
5,550
1,000
4,550
500
2,400
6,530
1,050

5,500

$\begin{array}{cc}18,500 & +1.1 \\ 1,350 & -3.3 \\ 400 & 0 \\ 400 & 0 \\ 550 & -9.1 \\ 17,150 & +1.5 \\ 550 & 0 \\ 1,050 & +4.8 \\ 5,950 & 0 \\ 1,000 & -10.7 \\ 4,950 & +3.1 \\ 550 & 0 \\ 2,450 & +2.1 \\ 6,600 & +2.3 \\ 1,000 & +10.5 \\ 5,600 & +1.0\end{array}$

a Based on annual average change from 1971 to 1972 , from 1972 to 1973 , etc. 
Table 6.7. (Continued)

\begin{tabular}{|c|c|c|c|c|c|c|c|c|c|c|}
\hline \multirow[b]{2}{*}{ Employment category } & \multicolumn{4}{|c|}{1972} & \multicolumn{2}{|l|}{$\begin{array}{l}\% \\
\mathrm{C} \\
\mathrm{h} \\
\mathrm{a} \\
\mathrm{n} \\
\mathrm{g}\end{array}$} & \multicolumn{2}{|c|}{1973} & \multirow[b]{2}{*}{ Dec. } & \multirow[t]{2}{*}{$\begin{array}{l}\% \\
\mathrm{C} \\
\mathrm{h} \\
\mathrm{a} \\
\mathrm{n} \\
\mathrm{g} \\
\mathrm{e}\end{array}$} \\
\hline & Mar. & Jun. & Sep. & Dec. & $\mathrm{e}$ & Mar. & Jun. & Sep. & & \\
\hline Civilian labor force & 28,950 & 29,000 & 25,450 & 27,750 & +4.4 & 28,800 & 29,000 & 26,300 & 30,450 & +2.0 \\
\hline $\begin{array}{l}\text { Unemployment } \\
\text { Unemployment rate, seasonal adjustment } \\
\text { Unemployment rate }\end{array}$ & $\begin{array}{r}3,500 \\
11.0 \\
12.1\end{array}$ & $\begin{array}{r}2,800 \\
10.6 \\
9.7\end{array}$ & $\begin{array}{r}2,400 \\
10.0 \\
9.4\end{array}$ & $\begin{array}{r}3,100 \\
10.0 \\
11.2\end{array}$ & $\begin{array}{l}-6.4 \\
-7.0 \\
-7.0\end{array}$ & $\begin{array}{r}3,450 \\
11.0 \\
12.0\end{array}$ & $\begin{array}{r}3,300 \\
12.4 \\
11.4\end{array}$ & $\begin{array}{r}3,300 \\
13.1 \\
12.5\end{array}$ & $\begin{array}{r}3,850 \\
11.4 \\
12.6\end{array}$ & $\begin{array}{l}+15.3 \\
+13.3 \\
+13.3\end{array}$ \\
\hline $\begin{array}{l}\text { Total civilian employment } \\
\text { Self employed } \\
\text { Domestics }\end{array}$ & $\begin{array}{r}25,450 \\
2,450 \\
350\end{array}$ & $\begin{array}{r}26,200 \\
2,450 \\
350\end{array}$ & $\begin{array}{r}23,050 \\
2,450 \\
350\end{array}$ & $\begin{array}{r}24,650 \\
2,450 \\
350\end{array}$ & $\begin{array}{c}+1.6 \\
0 \\
0\end{array}$ & $\begin{array}{r}25,350 \\
2,450 \\
350\end{array}$ & $\begin{array}{r}25,700 \\
2,450 \\
350\end{array}$ & $\begin{array}{r}23,000 \\
2,450 \\
350\end{array}$ & $\begin{array}{r}26,600 \\
2,450 \\
350\end{array}$ & $\begin{aligned} &+ 0.4 \\
& 0 \\
& 0\end{aligned}$ \\
\hline \multicolumn{11}{|l|}{ Job based } \\
\hline $\begin{array}{l}\text { Nonagricultural wage and salary } \\
\text { Manufacturing } \\
\text { Food and kindred } \\
\text { Stone, clay, and glass } \\
\text { Other manufacturing } \\
\text { Nonmanufacturing } \\
\text { Construction } \\
\text { Transportation, communications, utilities } \\
\text { Trade } \\
\text { Wholesale } \\
\text { Retai1. } \\
\text { Finances, insurance, and real estate } \\
\text { Services } \\
\text { Government } \\
\text { Federa1 } \\
\text { State and local }\end{array}$ & $\begin{array}{r}18,900 \\
1,350 \\
500 \\
400 \\
450 \\
17,550 \\
600 \\
1,100 \\
5,800 \\
1,150 \\
4,650 \\
550 \\
2,650 \\
6,850 \\
1,000 \\
5,850\end{array}$ & $\begin{array}{r}19,650 \\
1,700 \\
750 \\
400 \\
550 \\
17,950 \\
750 \\
1,100 \\
6,050 \\
1,300 \\
4,750 \\
550 \\
2,600 \\
6,900 \\
1,000 \\
5,900\end{array}$ & $\begin{array}{r}19,200 \\
1,500 \\
550 \\
450 \\
500 \\
17,700 \\
650 \\
1,050 \\
5,750 \\
1,050 \\
4,700 \\
550 \\
2,700 \\
7,000 \\
1,050 \\
5,950\end{array}$ & $\begin{array}{r}19,450 \\
1,350 \\
400 \\
450 \\
500 \\
18,100 \\
600 \\
1,100 \\
6,300 \\
1,100 \\
5,200 \\
550 \\
2,600 \\
6,950 \\
1,000 \\
5,950\end{array}$ & $\begin{array}{c}+3.3 \\
0 \\
0 \\
0 \\
0 \\
+3.5 \\
+9.1 \\
-4.6 \\
0 \\
-12.0 \\
+3.3 \\
+10.5 \\
+10.4 \\
+4.6 \\
-4.8 \\
+6.3\end{array}$ & $\begin{array}{r}20,300 \\
1,600 \\
550 \\
400 \\
650 \\
18,700 \\
650 \\
1,100 \\
6,450 \\
1,400 \\
5,050 \\
600 \\
2,700 \\
7,200 \\
1,000 \\
6,200\end{array}$ & $\begin{array}{r}20,850 \\
1,850 \\
750 \\
400 \\
700 \\
19,000 \\
650 \\
1,200 \\
6,650 \\
1,700 \\
4,950 \\
550 \\
2,650 \\
7,300 \\
1,000 \\
6,300\end{array}$ & $\begin{array}{r}20,200 \\
1,600 \\
500 \\
400 \\
700 \\
18,600 \\
600 \\
1,150 \\
6,300 \\
1,400 \\
4,900 \\
550 \\
2,650 \\
7,350 \\
1,000 \\
6,350\end{array}$ & $\begin{array}{r}21,250 \\
1,650 \\
550 \\
350 \\
750 \\
19,600 \\
650 \\
1,300 \\
6,850 \\
1,500 \\
5,350 \\
550 \\
2,650 \\
7,600 \\
1,000 \\
6,600\end{array}$ & $\begin{array}{r}+7.6 \\
+17.2 \\
+9.1 \\
0 \\
+40.0 \\
+5.8 \\
+8.3 \\
+9.5 \\
+11.2 \\
+31.8 \\
+\quad 6.4 \\
0 \\
\\
+5 \\
+5.8 \\
0 \\
+6.8\end{array}$ \\
\hline
\end{tabular}


Tab1e 6.7. (Continued)

\begin{tabular}{|c|c|c|c|c|c|c|c|c|c|c|c|}
\hline Employment category & Mar. & Jun. & Sep. & Dec. & $\begin{array}{l}\% \\
\mathrm{C} \\
\mathrm{h} \\
\mathrm{a} \\
\mathrm{n} \\
\mathrm{g} \\
\mathrm{e}\end{array}$ & Mar. & Jun. & Sep. & Dec. & $\begin{array}{c}\% \\
\text { Change }\end{array}$ & $\begin{array}{l}\text { Net } \\
\text { change } \\
1970- \\
1975\end{array}$ \\
\hline Civilian labor force & 30,100 & 31,750 & 28,550 & 32,750 & +7.9 & 31,350 & 33,400 & 30,200 & 33,700 & +4.6 &.+24.5 \\
\hline $\begin{array}{l}\text { Unemployment } \\
\text { Unemployment rate, seasonal adjustment } \\
\text { Unemployment rate }\end{array}$ & $\begin{array}{r}3,300 \\
10.2 \\
11.0\end{array}$ & $\begin{array}{r}3,450 \\
11.8 \\
10.9\end{array}$ & $\begin{array}{r}3,950 \\
14.4 \\
13.8\end{array}$ & $\begin{array}{r}4,300 \\
11.9 \\
13.1\end{array}$ & $\begin{array}{l}+8.8 \\
+0.8 \\
+0.8\end{array}$ & $\begin{array}{r}3,900 \\
11.6 \\
12.4\end{array}$ & $\begin{array}{r}5,900 \\
19.2 \\
17.7\end{array}$ & $\begin{array}{r}5,350 \\
18.3 \\
17.7\end{array}$ & $\begin{array}{r}4,550 \\
12.2 \\
13.5\end{array}$ & $\begin{array}{l}+29.7 \\
+24.8 \\
+23.1\end{array}$ & $\begin{array}{l}+100.0 \\
+62.4 \\
+44.7\end{array}$ \\
\hline $\begin{array}{l}\text { Total civilian employment } \\
\text { Self employed } \\
\text { Domestics }\end{array}$ & $\begin{array}{r}26,800 \\
2,450 \\
350\end{array}$ & $\begin{array}{r}28,300 \\
2,450 \\
350\end{array}$ & $\begin{array}{r}24,600 \\
2,450 \\
350\end{array}$ & $\begin{array}{r}28,450 \\
2,450 \\
350\end{array}$ & $\begin{array}{l}+7.8 \\
0 \\
0\end{array}$ & $\begin{array}{r}27,450 \\
2,450 \\
350\end{array}$ & 27,500 & 24,850 & 29,150 & $\begin{aligned} &+ 2.0 \\
& 0 \\
& 0\end{aligned}$ & $\begin{array}{l}+17.8 \\
+\quad 2.1 \\
\quad 0\end{array}$ \\
\hline \multicolumn{12}{|l|}{ Job based } \\
\hline 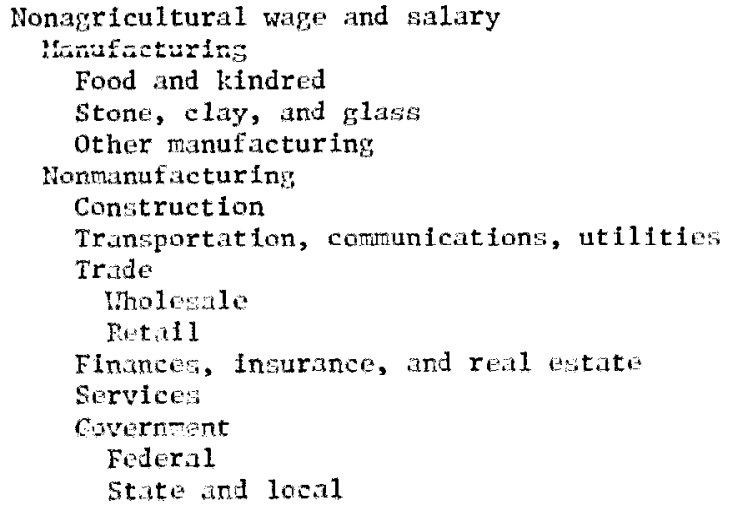 & $\begin{array}{r}21,400 \\
1,650 \\
500 \\
350 \\
800 \\
19,750 \\
650 \\
1,300 \\
6,800 \\
1,850 \\
4,950 \\
650 \\
2,800 \\
7,550 \\
1,050 \\
6,500\end{array}$ & $\begin{array}{r}21,850 \\
2,000 \\
700 \\
350 \\
950 \\
19,850 \\
700 \\
1,450 \\
6,650 \\
1,600 \\
5,050 \\
650 \\
2,800 \\
7,600 \\
1,000 \\
6,600\end{array}$ & $\begin{array}{r}21,300 \\
2,000 \\
650 \\
350 \\
1,000 \\
19,300 \\
600 \\
1,350 \\
6,400 \\
1,350 \\
5,050 \\
600 \\
2,800 \\
7,550 \\
1,000 \\
6,550\end{array}$ & $\begin{array}{r}22,250 \\
1,900 \\
550 \\
350 \\
1,000 \\
20,350 \\
600 \\
1,450 \\
7,250 \\
1,950 \\
5,300 \\
600 \\
2,800 \\
7,650 \\
1,000 \\
6,650\end{array}$ & $\begin{array}{r}+5.1 \\
+8.8 \\
0 \\
-12.5 \\
+28.5 \\
+4.8 \\
0 \\
+17.4 \\
+3.9 \\
+13.8 \\
+1.0 \\
+9.1 \\
+5.7 \\
+2.1 \\
\\
+4 \\
+4.0\end{array}$ & $\begin{array}{r}22,350 \\
1,900 \\
500 \\
300 \\
1,100 \\
20,450 \\
600 \\
1,400 \\
6,950 \\
1,850 \\
5,100 \\
650 \\
3,000 \\
7,850 \\
1,000 \\
6,850\end{array}$ & $\begin{array}{r}22,750 \\
2,150 \\
750 \\
300 \\
1,100 \\
20,600 \\
700 \\
1,500 \\
6,950 \\
1,800 \\
5,150 \\
650 \\
2,950 \\
7,850 \\
1,000 \\
6,850\end{array}$ & $\begin{array}{r}22,250 \\
1,900 \\
550 \\
350 \\
1,000 \\
20,350 \\
700 \\
1,350 \\
6,750 \\
1,450 \\
5,300 \\
650 \\
3,050 \\
7,850 \\
1,000 \\
6,850\end{array}$ & $\begin{array}{r}23,650 \\
1,700 \\
600 \\
350 \\
750 \\
21,950 \\
600 \\
1,300 \\
8,500 \\
2,700 \\
5,800 \\
600 \\
3,000 \\
7,950 \\
1,100 \\
6,850\end{array}$ & $\begin{array}{c}+6.0 \\
+2.7 \\
0 \\
-7.1 \\
+11.1 \\
+5.3 \\
0 \\
0 \\
+4.5 \\
+12.1 \\
+5.9 \\
+4.2 \\
+7.1 \\
+4.3 \\
+2.5 \\
+4.6\end{array}$ & $\begin{array}{r}+25.3 \\
+26.7 \\
+\quad 9.1 \\
-18.8 \\
+81.8 \\
+24.0 \\
+18.2 \\
+28.6 \\
+37.9 \\
+32.1 \\
+21.6 \\
+25.0 \\
+27.7 \\
+22.1 \\
+7.9 \\
+24.5\end{array}$ \\
\hline
\end{tabular}


to actually decline over that

6-yr period. Federal government employment, as is very common when at a distance from major federal installations, rose very slowly; however, state and local government employment increased by $24.5 \%$ or at an even pace with most of the private sector, Unemployment in Imperial County, as in most areas of the state, climbed dramatically over this period. It is noteworthy, however, that the largest single rise in unemployment occurred in 1975 - a period when most areas were beginning to reduce local unemployment rates. Futhermore, the unemployment rate in Imperial County throughout this entire period is higher than might be expected. The employment of Mexican green card holders in various labor fields (see subsection below on Mexican labor force) should act as a partial buffer against a high county unemployment rate since, although unemployed, green card holders are not counted in unemployment figures. Thus unemployment rates should not reflect total unemployment, but rather total unemployment less green card holder unemployment.

Some perspective on the stability of various industries can be obtained from Table 6.7. Wholesale activity was the least stable over this 6-yr period within the Imperial County economy with annual percentage changes of : $-10 \%,-12 \%,+31.8 \%$, and $+12.1 \%$. The general class of other manufacturing followed closely behind with changes of $-9.1 \%, 0 \%,+40.0 \%,+28.5 \%$, and $+11.1 \%$. The large 1973 jump is, again, the result of the advent of new industries listed in Table 6.3. Manufacturing as a whole was much more stable than other manufacturing primarily because of the size of the local food and kindred manufacturing force. Construction, traditionally a nonstable sector, was unusually stable in Imperial County over this period, showing good gain in 1973 and 1974 and no growth in the other 3 years.

In addition to employment data collected industry by industry by the Department of Commerce, data are also collected as part of the census survey for employment by job class. The data from the 1970 census are shown on Table 6.8. While this type of data complements SIC employment figures, no direct industry-by-industry comparison is possible with the data in their present form. The SIC employment figures give the number of persons employed by a given activity regardless of their job class (clerical, craftwork, manager, etc.), and the census data present total county employment for each job class regardless of the industry employing the individuals. 
Table 6.8. Imperial County employment by job cl.ass (1970). 14

Job class
1970 Employment

25,257

23,479

2,489

94

329

986

2,418

1,597

3,592

2, 771

704

986

47

1,033

1,573

1,150

1,057

2,817

352

$3,663^{*}$ $\pi$

$100 \%$

10.6

0.4

1.4

4.2

10.3

6.8

15.3

11.8

3.0

4.2

0.2

4.4

6.7

4.9

4.5

12.0

1.5

15.6

*Does not include most migrant labor or Mexican green card labor.

Unemployment Characteristics and Current Employment Prospects.

Unemployment and employment levels for Imperial County are plotted together in Fig. 6.1 for 1970 through 1975 to show their relationship. Major annual drops in employment occur in late summer. These employment reductions correspond not to periods of increased registe:red unemployment, but rather to the annual low point for registered employment. Two factors can partially explain this phenomenon. First, most summer student help terminates in late summer and returns to school rather than entering unemployment rolls. Second, and by 

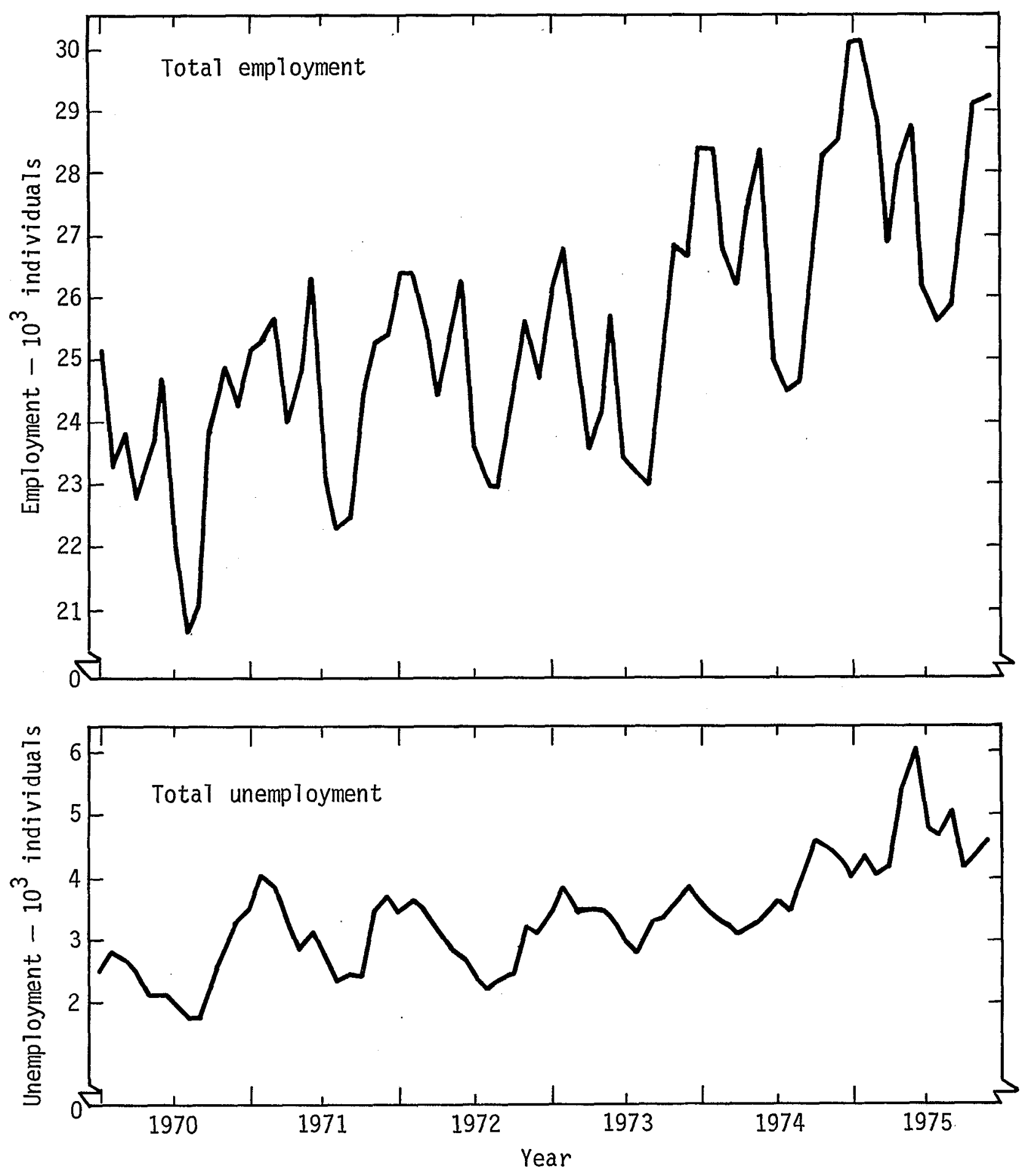

Fig. 6.1. Total employment and unemployment for Imperial County, 1970 to 1975. 
far the larger effect, is an annual outmigration of transient, seasonal agricultural labor. Nonagricultural unemployment for the county shows very low annual fluctuations. Some statistics on registered unemployed persons as of 31 January 1976 are shown in Table 6.9. This breakdown shows no unique or unusual trends in Imperial County compared to other California counties. There is a high percentage of Spanish Americans on the county unemployment rolls; however their presence is not an intrinsic characteristic of the county economy but more significantly a function of its location. Seasonal and migrant farmworkers are not uncommon in an agriculturally based county.

Employment and labor opportunity trends are summarized monthly and projected annually by the office of Employment Data and Research (EDR). Their data showed that in 1975 the employed labor force increased by 2000 persons, while net agricultural employment dropped. This large increase in nonagricultural employment was counterbalanced by an unemployment increase to $14.3 \%$ of the work force. Projections for 1976 show a slowed rate of net nonagricultural employment gain and, consistent with the projections made in Section 6.2 , continued net agricultural employment reductions. Combined with the already high unemployment rate, this means that there vill be an excess in total county labor supply throughout 1976 and into 1977.

Analyzing these EDR trends and projections on an industry-by-industry level, late 1975 employment increases vere attributable to large increases in retail employment followed by smaller gains in construction, wholesale, transportation, utilities, and government. Manufacturing and finance remained unchanged while the services sector declined in employment. Steady growth is projected in retail and, to a lesser extent, in wholesale sectors throughout the foreseeable future. Employment levels for services and for finance, insurance and real estate are expected to be closely tied to population changes. If county population increases follow the grovth pattern of the past 5 years, nodest, steady increasea will occur in service related employment. lanufacturing employment changes should be dependent on the creation of new industrial activities rather than on expansion of cristing facilities, with conetruction employment being somewhat dependent on the influz of new industry. Transportation, communication and utilities are projected to remain relatively stable at curxent employment levels. 
Table 6.9. Imperial County unemployment characteristics as of 31 January 1976.13

\begin{tabular}{|c|c|c|c|c|c|c|c|c|c|c|c|c|c|c|c|}
\hline \multirow{2}{*}{$\begin{array}{l}\text { Characteristics } \\
\text { of applicants }\end{array}$} & \multirow[t]{2}{*}{ Total } & \multirow[t]{2}{*}{ Female } & \multirow{2}{*}{$\begin{array}{l}\text { Econ- } \\
\text { omic- } \\
\text { ally } \\
\text { dis- } \\
\text { advan- } \\
\text { taged }\end{array}$} & \multirow{2}{*}{$\begin{array}{l}\text { Handi- } \\
\text { capped }\end{array}$} & \multicolumn{3}{|c|}{ Minority } & \multirow{2}{*}{$\begin{array}{l}\text { Wel- } \\
\text { fare }\end{array}$} & \multicolumn{3}{|c|}{ Veteran } & \multirow{2}{*}{$\begin{array}{l}\text { UI } \\
\text { claim- } \\
\text { ant }\end{array}$} & \multirow{2}{*}{$\begin{array}{l}\text { Sea- } \\
\text { sonal } \\
\text { worker }\end{array}$} & \multicolumn{2}{|c|}{ Age } \\
\hline & & & & & Total & Black & $\begin{array}{l}\text { Span- } \\
\text { ish } \\
\text { Amer- } \\
\text { ican }\end{array}$ & & Total & $\begin{array}{l}\text { Viet- } \\
\text { nam } \\
\text { era }\end{array}$ & $\begin{array}{l}\text { Spe- } \\
\text { cial } \\
\text { Viet- } \\
\text { nam } \\
\text { era }\end{array}$ & & & $\begin{array}{l}\text { Under } \\
22\end{array}$ & $\begin{array}{l}45 \text { or } \\
\text { older }\end{array}$ \\
\hline B & c & D & E & $\mathrm{F}$ & G & H & I & $\mathrm{J}$ & $\mathrm{K}$ & $\mathrm{L}$ & M & $\mathrm{N}$ & 0 & $P$ & Q \\
\hline $\mathrm{Age}$ & 2216 & 942 & 1238 & 135 & 1499 & 118 & 1366 & 717 & 398 & 221 & 23 & 747 & 530 & 387 & 550 \\
\hline Under 20 & 175 & 58 & 96 & 3 & 114 & 9 & 104 & 35 & 8 & 8 & 1 & 48 & 37 & 175 & 0 \\
\hline $20-21$ & 212 & 80 & 110 & 2 & 141 & 15 & 126 & 37 & 32 & 30 & 3 & 71 & 37 & 212 & 0 \\
\hline $22-24$ & 276 & 90 & 119 & - & 192 & 15 & 176 & 57 & 65 & 64 & 10 & 1.11 & 61. & 0 & 0 \\
\hline $25-29$ & 326 & 119 & 1.55 & 13 & 224 & 19 & 204 & 97 & 73 & 71 & 5 & 125 & 76 & 0 & 0 \\
\hline $30-39$ & 468 & 251 & 293 & 29 & 297 & 27 & 264 & 208 & 66 & 34 & 2 & 148 & 93 & 0 & 0 \\
\hline $40-44$ & 209 & 111 & 147 & 20 & 159 & 10 & 147 & 101 & 24 & 6 & 2 & 70 & 70 & 0 & 0 \\
\hline $45-54$ & 373 & 179 & 233 & 42 & 258 & 14 & 242 & 141 & 86 & 7 & 0 & 122 & 98 & 0 & 373 \\
\hline $55-64$ & 151 & 49 & 78 & 19 & 99 & 9 & 90 & 41 & 37 & 1 & 0 & 41 & 46 & 0 & 151 \\
\hline 65 and over & 26 & 5 & 7 & 3 & 15 & 0 & 13 & 0 & 7 & 0 & 0 & 11 & 12 & 0 & 26 \\
\hline Sex & 2216 & - & 1238 & 135 & 1499 & 118 & 1366 & 717 & 398 & 221 & 23 & 747 & 530 & 387 & 550 \\
\hline Male & 1274 & 0 & 645 & 92 & 843 & 59 & 776 & 301 & 393 & 218 & 23 & 476 & 359 & 249 & 317 \\
\hline Female & 942 & - & 593 & 43 & 656 & 59 & 590 & 416 & 5 & 3 & 0 & 271 & 171 & 136 & 233 \\
\hline Highest school grade & 2216 & 942 & 1238 & 135 & 1499 & 118 & 1366 & 717 & 398 & 221 & 23 & 747 & 530 & 387 & 550 \\
\hline $0-7$ & 667 & 323 & 460 & 48 & 627 & 14 & 610 & 289 & 26 & 2 & 0 & 226 & 294 & 24 & 311 \\
\hline $8-11$ & 720 & 295 & 444 & 54 & 485 & 45 & 436 & 267 & $1.17^{\circ}$ & 56 & 9 & 223 & 163 & 178 & 129 \\
\hline 12 & 573 & 230 & 241 & 17 & 266 & 42 & 220 & 123 & 173 & 120 & 8 & 199 & 54 & 147 & 73 \\
\hline Over 12 & 256 & 94 & 93 & 16 & 121 & 17 & 100 & 38 & 82 & 43 & 6 & 99 & 19 & 38 & 37 \\
\hline Residence & 2216 & 942 & 1238 & 135 & 1499 & 118 & 1366 & 717 & 398 & 221 & 23 & 747 & 530 & 387 & 550 \\
\hline Urban & 1907 & 802 & 1084 & 110 & 1329 & 113 & 1206 & 633 & 344 & 194 & 21 & 637 & 447 & 332 & 474 \\
\hline Rural & 309 & 140 & 154 & 25. & 170 & 5 & 160 & 84 & 54 & 27 & 2 & 110 & 83 & 55 & 76 \\
\hline
\end{tabular}


Table 6.9. (Concluded)

\begin{tabular}{|c|c|c|c|c|c|c|c|c|c|c|c|c|c|c|c|}
\hline \multirow{2}{*}{$\begin{array}{c}\text { Characteristics } \\
\text { of applicants }\end{array}$} & \multirow[t]{2}{*}{ Total } & \multirow[t]{2}{*}{ Female } & \multirow{2}{*}{$\begin{array}{l}\text { Econ- } \\
\text { omic- } \\
\text { ally } \\
\text { dis- } \\
\text { advan- } \\
\text { taged }\end{array}$} & \multirow{2}{*}{$\begin{array}{l}\text { Handi- } \\
\text { capped }\end{array}$} & \multicolumn{3}{|c|}{ Minority } & \multirow{2}{*}{$\begin{array}{l}\text { We1- } \\
\text { fare }\end{array}$} & \multicolumn{3}{|c|}{ Veteran } & \multirow{2}{*}{$\begin{array}{l}\quad \text { UI } \\
\text { claim- } \\
\text { ant }\end{array}$} & \multirow{2}{*}{$\begin{array}{l}\text { Sca- } \\
\text { sonal } \\
\text { worker }\end{array}$} & \multicolumn{2}{|c|}{ Age } \\
\hline & & & & & Total & Black & $\begin{array}{l}\text { Span- } \\
\text { ish } \\
\text { Ameri- } \\
\text { can }\end{array}$ & & Total & $\begin{array}{l}\text { Vict- } \\
\text { nam } \\
\text { era }\end{array}$ & $\begin{array}{l}\text { Spc- } \\
\text { cial } \\
\text { Viet- } \\
\text { nam } \\
\text { era }\end{array}$ & & & $\begin{array}{l}\text { Under } \\
22\end{array}$ & $\begin{array}{l}45 \text { or } \\
\text { older }\end{array}$ \\
\hline B & $\mathrm{C}$ & $\mathrm{D}$ & $\mathrm{E}$ & $\mathrm{F}$ & G & $\mathrm{H}$ & 1 & $\mathrm{~J}$ & $\mathrm{~K}$ & L & $\mathrm{M}$ & $N$ & 0 & $P$ & $Q$ \\
\hline Ethnic group & 2216 & 942 & 1238 & 135 & 1499 & 118 & 1366 & 717 & 398 & 221 & 23 & 747 & 530 & 387 & 550 \\
\hline White & 2057 & 858 & 1131 & 128 & 1354 & 0 & 1354 & 635 & 377 & 210 & 22 & 708 & 514 & 361 & 516 \\
\hline Black & 118 & 59 & 76 & 5 & 118 & - & 3 & 53 & 16 & 8 & 1 & 30 & 13 & 24 & 23 \\
\hline American Indian & 10 & 5 & 9 & 1 & 10 & 0 & 2 & 9 & 2 & 2 & 0 & 1 & 1 & 0 & 0 \\
\hline other & 15 & 6 & 6 & 1 & 15 & 0 & 5 & 3 & 3 & 1 & 0 & 5 & 2 & 1 & 8 \\
\hline Ina & 16 & 14 & 16 & 0 & 2 & 0 & 2 & 16 & 0 & 0 & 0 & 3 & 0 & 1 & 3 \\
\hline Spanish American & 1366 & 590 & 847 & 71 & 1366 & 3 & - & 485 & 138 & 90 & 11 & 491 & 455 & 230 & 345 \\
\hline UI claimant & 747 & 271 & 320 & 27 & 523 & 30 & 491 & 138 & 133 & 85 & 7 & - & 218 & 119 & 174 \\
\hline State & 581 & 226 & 229 & 22 & 400 & 24 & 375 & 111 & 85 & 45 & 2 & 581 & 146 & 87 & 130 \\
\hline Veteran & 398 & 5 & 166 & 40 & 158 & 16 & 138 & 53 & - & 221 & 23 & 133 & 51 & 40 & 130 \\
\hline Fiecentiy depataled & 130 & 2 & 53 & 5 & 62 & 5 & 56 & 12 & 130 & 130 & 15 & 60 & 16 & 34 & 7 \\
\hline Vietnam era & 221 & 3 & 90 & 13 & 101 & 8 & 90 & 34 & 221 & - & 22 & 85 & 22 & 38 & 8 \\
\hline Special Vietnam era & 22 & 0 & 15 & 1 & 12 & 1 & 11 & 5 & 22 & 22 & - & 7 & 7 & 4 & 0 \\
\hline Disabled & 20 & 0 & 9 & 15 & 5 & 0 & 5 & 2 & 20 & 10 & 1 & 5 & 3 & 1 & 9 \\
\hline Handicapped & 135 & 43 & 100 & - & 78 & 5 & 71 & 81 & 40 & 13 & 2 & 27 & 23 & 5 & 64 \\
\hline $\begin{array}{l}\text { Economically } \\
\text { disadvantaged }\end{array}$ & 1238 & - & 1238 & 100 & 934 & 76 & 847 & 679 & 166 & 90 & 15 & 320 & 291 & 206 & 318 \\
\hline Food stamp & 409 & 179 & 378 & 50 & 293 & 27 & 266 & 233 & 39 & 23 & 5 & 94 & 95 & 64 & 117 \\
\hline Welfare & 717 & 416 & 679 & 81 & 548 & 53 & 485 & - & 53 & 34 & 5 & 138 & 89 & 72 & 182 \\
\hline WIN & 693 & 407 & 655 & 80 & 533 & 53 & 470 & 693 & 48 & 31 & 4 & 135 & 32 & 68 & 178 \\
\hline CETA & 9 & 4 & $y$ & 1 & $b$ & 0 & 6 & 8 & 1 & 1 & 0 & 1 & 0 & 3 & 2 \\
\hline Seasonal farrworker & 5.70 & 171 & 291 & 23 & 469 & 13 & 455 & 89 & 31 & 22 & 7 & 218 & - & 74 & 156 \\
\hline Migrant farmoryer & 223 & 63 & 113 & 7 & 191 & 3 & 157 & 23 & 26 & 11 & 2 & et & 223 & 31 & to \\
\hline
\end{tabular}


As of March 1976, the State Employment Development Department (EDD) forecast little activity in hiring for professional skills in a11 employment sectors. Clerical placement prospects are good to fair in retail, wholesale, and government sectors, and slow in other sectors. $^{15}$ Sales and general service employment is projected to be slow because of excess labor supplies. Specialized industrial and construction workers are relatively easy to place, especially in $\mathrm{El}$ Centro and Imperial; however most construction and manufacturing jobs are unionized and are therefore hired through union halls. Little data on these types of positions are available through EDD.

Mexican Labor Force in Imperial County

Staff workers for the Community Services Agency sponsored organization Campesinos Unidos, which is based in Brawley; have gathered considerable unpublished data on the magnitude of the Mexican Labor Force in Imperial County. ${ }^{16}$ Robert E. Nilan, U.S. Immigration Service, Calexico, reported that approximately $20 \%$ of the Imperial Valley population are permanent U.S. resident aliens. This labor force is treated statistically as resident labor. Daily commuting green card holders, however, have a much greater impact on the county. This group acts as an impact buffer for Imperial County employment by absorbing a percentage of sudden changes in demand for employment, thus reducing the fluctuation in employment and unemployment felt by the county and 1ocal governments. The distribution of these green card workers throughout the county economy for a single 3 -month period is shown in Table 6.10. These figures are somewhat misleading since late summer is the annual employment low for agriculture. Unofficial county staff estimates have put peak agricultural green card employment at between 6500 and 8000 daily. To place this volume of traffic in better perspective, daily border crossings were tabulated at each border station and reported by Campesinos Unidos. ${ }^{16}$ Annual border crossings at Calexico ranged from 12.5 million in 1970 to 15.7 million in 1974. (Green card workers represent slightly over $14.0 \%$ of al1 border crossings.)

\section{Imperial County Agricultural Employment}

Agricultural employment data for both seasonal and regular hire labor is gathered for Imperial County by EDR. Their 1976 projection for most Imperial Valley crops is 
Table 6.10. Imperial County daily Mexican green card holder work force for July through Sept. 1975. a

Sector

Number of alien commuters (Mexican residence) identificd July, 1975 August, 1975 September, 1975

$\begin{array}{lrrr}\text { Agriculture } & 4,901 & 4,911 & 4,953 \\ \text { Industry } & 126 & 138 & 153 \\ \text { Construction } & 44 & 44 & 42 \\ \text { Sales/service } & 238 & 243 & 248 \\ \text { Domestic } & 38 & 37 & 39 \\ \text { Other } & 37 & 37 & 37 \\ & 5,384 & 5,410 & 5,472\end{array}$

${ }^{a}$ From Pepe Rena1do, Imperial Valley Characterization for Campesinos Unido: (unpublished). 16

shown in Table 6.11. A simple breakdown into regular hired, farmer/ owner and unpaid family, and seasonal workers for some crops is given in Table 6.12. The nonseasonal workforce for most crops is minimal. No shortage of agricultural labor to meet these large seasonal demands is forecast for 1976 to 1977.

The only long term trend in county agricultural employment is a general, steady downward trend. In their Ultimate Land Use Plan, ${ }^{3}$ the county planning staff reports a $48 \%$ decrease in agricultural employment over the period of 1960 to 1972 . With a continued trend toward mechanization, this decline in agricultural employment should continue.

\subsection{IMPERIAL COUNTY ELECTRICAL ENERGY BUDGET}

Since geothermal resources will be used primarily for electrical power production, a brief characterization of Imperial County's electrical energy use patterns is pertinent. Table 6.13 shows the county electrical energy budget for 1972, 1974, and 1975. Table 6.14 shows the distribution of sales and use of electrical energy within the county and the relative rank of Imperial County in 1972 among the 58 California counties for each sales and use 
Table 6.11. Imperial County total agricultural employment ${ }^{a}$ by crop by month projected for 1976.17

\begin{tabular}{|c|c|c|c|c|c|c|c|c|c|c|c|c|}
\hline Crop & Jan & Feb & Mar & Apr & May & Jun & Ju1 & Aug & Sept & oct & Nov & Dec \\
\hline \multicolumn{13}{|l|}{ Vegetable } \\
\hline Carrot & 160 & 150 & 165 & 155 & 120 & 70 & 0 & 0 & 0 & 0 & 0 & 90 \\
\hline Onion & 100 & 250 & 150 & 40 & 600 & 450 & 50 & 0 & 0 & 0 & 60 & 80 \\
\hline Broccoli & 150 & 50 & 0 & 0 & 0 & 0 & 0 & 0 & 10 & 50 & 60 & 60 \\
\hline Other & 950 & 750 & 480 & 360 & 225 & 225 & 170 & 190 & 240 & 385 & 775 & 950 \\
\hline \multicolumn{13}{|l|}{ Field crops } \\
\hline Sugar beets & 450 & 550 & 600 & 550 & 425 & 500 & 275 & 0 & 350 & 1,200 & 1,200 & 600 \\
\hline Cotton & 175 & 100 & 0 & 10 & 500 & 450 & 325 & 170 & 30 & 10 & 375 & 325 \\
\hline Livestock & 1,000 & 1,000 & 1,000 & 1,000 & 1,000 & 1,000 & 1,000 & 1,000 & 1,000 & 1,000 & 1,000 & 1,000 \\
\hline Miscellaneous & 140 & 150 & 145 & 140 & 130 & 160 & 135 & 135 & 135 & 130 & 140 & 150 \\
\hline Specialty crops & 425 & 550 & 475 & 400 & 400 & 600 & 425 & 400 & 380 & 360 & 425 & 475 \\
\hline Total & 10,760 & 11,085 & 7,240 & 5,620 & 6,595 & 6,620 & 4,710 & 3,735 & 4,165 & 5,425 & 6,565 & 7,630 \\
\hline
\end{tabular}

Man months/month required labor. 
Table 6.12. Imperial County expected crop employment by employment category for 1976.17

\begin{tabular}{|c|c|c|c|c|c|c|}
\hline \multirow[b]{2}{*}{ Crop } & \multicolumn{3}{|c|}{ Total annual labor (man weeks) } & \multicolumn{3}{|c|}{ Pereent of total } \\
\hline & Farmer/family & $\begin{array}{l}\text { Regular } \\
\text { hire }\end{array}$ & Seasone 1 & Farmer/family & $\begin{array}{c}\text { Regular } \\
\text { hire }\end{array}$ & Seational \\
\hline Cotton & 340 & 460 & 9,510 & 3.3 & 4.5 & 92.2 \\
\hline Miscellaneous vegetables & 520 & 520 & 5,8801 & 7.5 & 7.5 & 85.0 \\
\hline Tomatoes & 580 & 870 & 6,150 & 7.7 & 11.6 & 82.0 \\
\hline Sugar beets & 660 & 1,140 & 26,040 & 2.4 & 4.1 & 93.1 \\
\hline Asparagus (harvest) & 190 & 190 & 11,490 & 1.6 & 1.6 & 96.8 \\
\hline Broccoli & 0 & 0 & 1,370 & 0 & 0 & 100.0 \\
\hline Lettuce & 510 & 1,340 & $\begin{array}{l}62,680 \\
43,7013\end{array}$ & 0.8 & 2.8 & 96.4 \\
\hline
\end{tabular}

category. Per capita rankings on Table 6.14 are rankings of each category $\mathrm{kWh}$ divided by county population. Per capita energy use in Imperial County ranks significantly higher than total energy use for most categories; as a result of large scale water pumping for irrigation, the public use category for Imperial County ranks 23 rd overal1 and 12 th on a per capita basis.

Imperial Irrigation District (IID) is responsible for generation, import, export, and sales of all electrical energy within the county. As of 1972, IID was 1 of 24 active electrical energy generating companies in California, producing 782,264,421 $\mathrm{kWh}$ or $0.61 \%$ of the state total. 18 IID was also 1 of 45 companies making direct electrical sales to consumers and sold $988,726,154 \mathrm{kWhr}$ or $0.71 \%$ of the state total. In addition they used 10,729,210 kWhr (primarily for irrigation water pumping) without direct consumer charge for a total sales and nonsales use (total use) of $999,455,364 \mathrm{kTh}$ or $0.69 \%$ of the state total. Their sales totaled $\$ 15,447,041$ in 1972 or $0.65 \%$ of the state total utility revenue from alos to ultimate consumers. Thus IID's average sales rate fell slightly below the state average for that ycar. IID system losses (1ine losses, conversion losses, etc.) vere 130,230 Whr or $11.52 \%$ of company total electrical energy. This figure is higher than state average, eapecially in light of the relatively small area covered by IID.

\subsection{IMPERIAL COUNTY ECONOMIC CHARACTERIZATION AND COIPARISON TO OTHER CALIFORNIA COUNTIES. \\ The information listed in the previous sections must be placed in some perspective for that information}


Table 6.13. Imperial County electrical energy budget for 1972, 1974, $1975 .{ }^{18}$

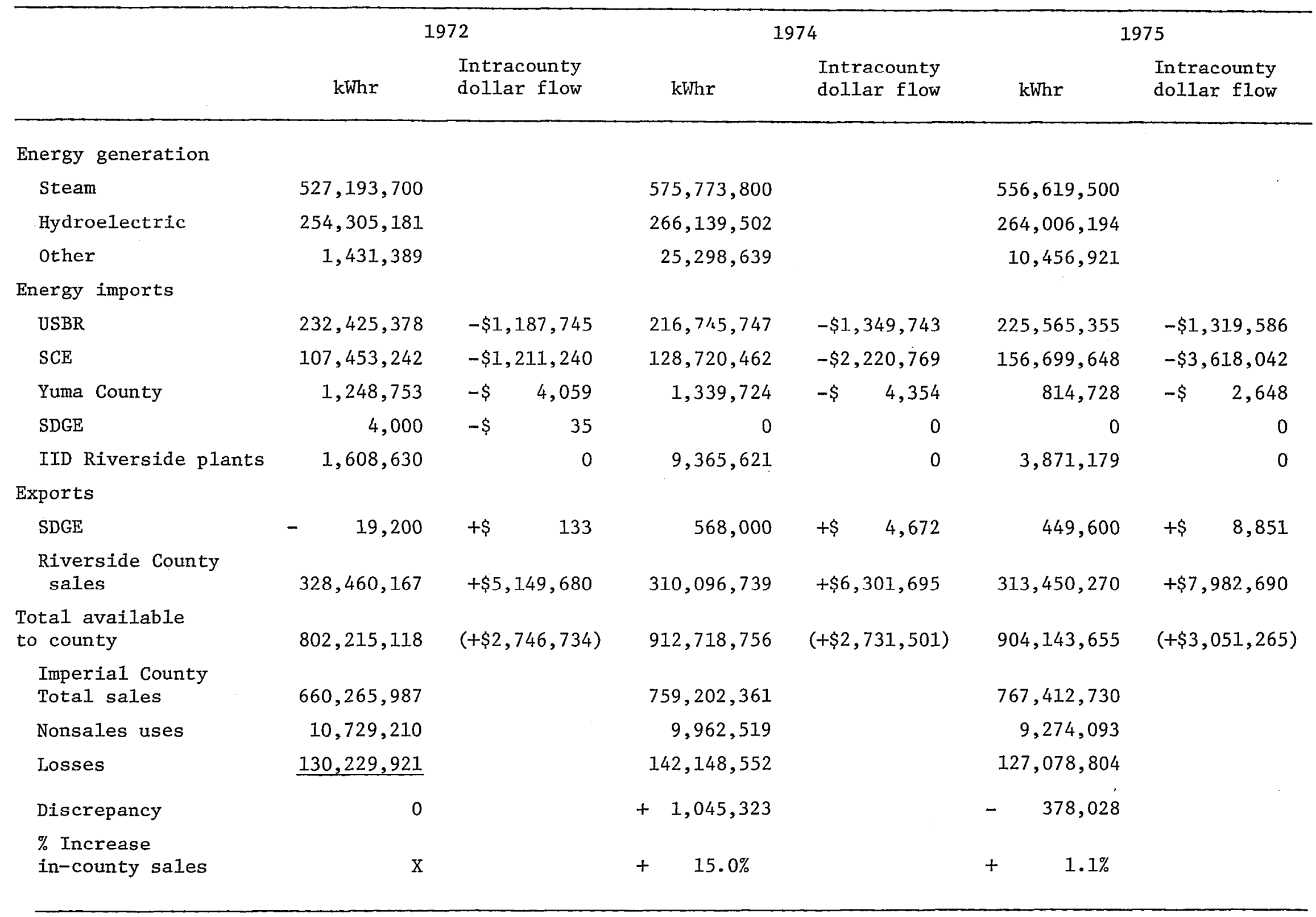


Table 6.14. Imperial County electrical energy sales and use data for $1972 .{ }^{18}$

\begin{tabular}{|c|c|c|c|c|}
\hline & County & total & & Per capita \\
\hline & $\mathrm{kW} \cdot \mathrm{hr}$ & $\%$ State total & Rank & Ranl: \\
\hline Population & & 0.38 & 32 & 32 \\
\hline $\begin{array}{l}\text { Residential electrical } \\
\text { sales }\end{array}$ & $276,337,423$ & 0.69 & 28 & 16 \\
\hline Commercial sales & $256,908,979$ & 0.54 & 30 & 15 \\
\hline Industrial sales & $76,738,153$ & 0.19 & 30 & 34 \\
\hline Sales to public sector & $50,525,443$ & 0.47 & 22 & 12 \\
\hline Total commercial use & $256,921,968$ & 0.54 & 30 & 16 \\
\hline Total industrial use & $76,738,153$ & 0.19 & 30 & 34 \\
\hline Total public use & $61,254,653$ & 0.39 & 23 & 12 \\
\hline Total sales & $660,950,998$ & 0.47 & 28 & 19 \\
\hline Total use & $671,252,176$ & 0.46 & 29 & 20 \\
\hline
\end{tabular}

to be usable for analytical purposes. County Business Pattern information for all 58 California counties was compiled for comparative analysis by calculating the following parameters:

- Location quotients were calculated for 83 SIC industries, including al1 1-digit, many 2-digit, and selected 3- and 4-digit SIC codes. A location quotient measures the presence of a given activity (SIC employment) in a county relative to the presence of that activity in the state as a whole. By definition, $\underset{\text { Tocation }}{\text { quotient }}=\frac{\frac{\text { County SIC employment }}{\text { County total employment }}}{\frac{\text { State SIC employment }}{\text { State total employment }}}$
A value of 1 indicates that an activity is present in a county to the same extent that it is present in the state as a whole. A very high value indicates that an activity is present in an inordinately high percentage in a county, that the county exports some or most of the products assoclated with that activity, and that the county is an improtant element in the state economy for that activity. Coefficients of localization vere calculated, based on data provided by Lofting ${ }^{2}$ for 1972 agricultural activity as whole, srop related employment, and beef, cattle, and other livestock: 
employment. Detailed data on Imperial County crop-by-crop employment have been collected and were presented in section 6.3. It would be desirable to calculate coefficients of localization for each of these specific crops; however such information has not been gathered for other counties or for the state as a whole. Thus, only general calculations are feasible at this time. While a location quotient compares the relative presence of each element within one subregion (county) to that for each element within a larger base economy (state), a coefficient of localization compares the relative presence of a single economic element (SIC) within each subregion of the base economy. Thus a coefficient of localization provides an index of the extent to which an industry is evenly spread among the counties of a state or is concentrated in only a few counties. The coefficient of localization varies from 0 to 1 , with low values indicating a high degree of diversification and high values indicating a high degree of localization. Primary activities (agriculture, mining, etc.) typically have fairly high coefficients of localization (greater than 0.4), while secondary activities such as manufacturing are in the 0.3 to 0.55 range, and tertiary activities (wholesale, retail, services) have very low values (typically around 0.2).

By definition,

$\begin{aligned} & \text { Coefficient } \\ & \text { localization }_{i}\end{aligned}=\frac{\sum_{j=1}^{n} d_{j}, \text { for all } d>0}{100}$ where $\mathrm{n}=$ number of counties within the state

and $\mathrm{d}_{j}=\frac{\text { County }_{j} \text { employment for } \text { SIC }_{i}}{\text { Total county }}$ $\frac{\text { State employment for } \mathrm{SIC}_{i}}{\text { Total state employment }}$.

- Indices of diversification were calculated for each county economy. This index is similar to the location quotient in that it indicates the distribution of activities in each subregion or county. However the index of diversification provides a single coefficient as a measure of the relative diversification of each county economy. The index of diversification varies from 0 to 1 with low values indicating a more diverse economy. This study uses the following form for calculation of 
the refined index of diversification (RID) :

$$
R I D=\frac{S A-A D}{A N-A D},
$$

where $\mathrm{SA}=$ crude index of diversification for the study area,

$\mathrm{AD}=$ crude index of absolute diversification, and

$\mathrm{AN}=$ crude index of absolute nondiversification

Isard ${ }^{19}$ has provided the methodology for computing crude indices of diversification. Typical state and county RID's are graphed with $\mathrm{AN}$ and $\mathrm{AD}$ in Fig. 6.2. RID's were calculated for each county with data from major industrial divisions only. The sectors covered are: agriculture; agricultural service; mining; contract construction; manufacturing; transportion and public utilities; wholesale; retail; finance, insurance, and real estate; and personal services. An RID graph covering these sectors for Imperial County is shown in Fig. 6.3.

Data for these three parameters are contained in Tables 6.15, 6.16, and 6.17 , respectively. RID elements for Imperial County are plotted with $\mathrm{AN}$ and $\mathrm{AD}$ in Fig. 6.3. Table 6.18 shows the SIC numbers analyzed in Tables 6.15 and 6.16 , and associated sector titles. The Imperial County sectors with exceptionally high location quotients are listed in Table 6.19 and compared to Kern and Fresno counties (two other asriculturally based counties) in Tab1 6.20 .

Location quotient data contained in Table 6.15 is relatively selfexplanatory. Imperial County is weak in mining and manufacturing, close to average in retal1, Einance and services, slightly above average

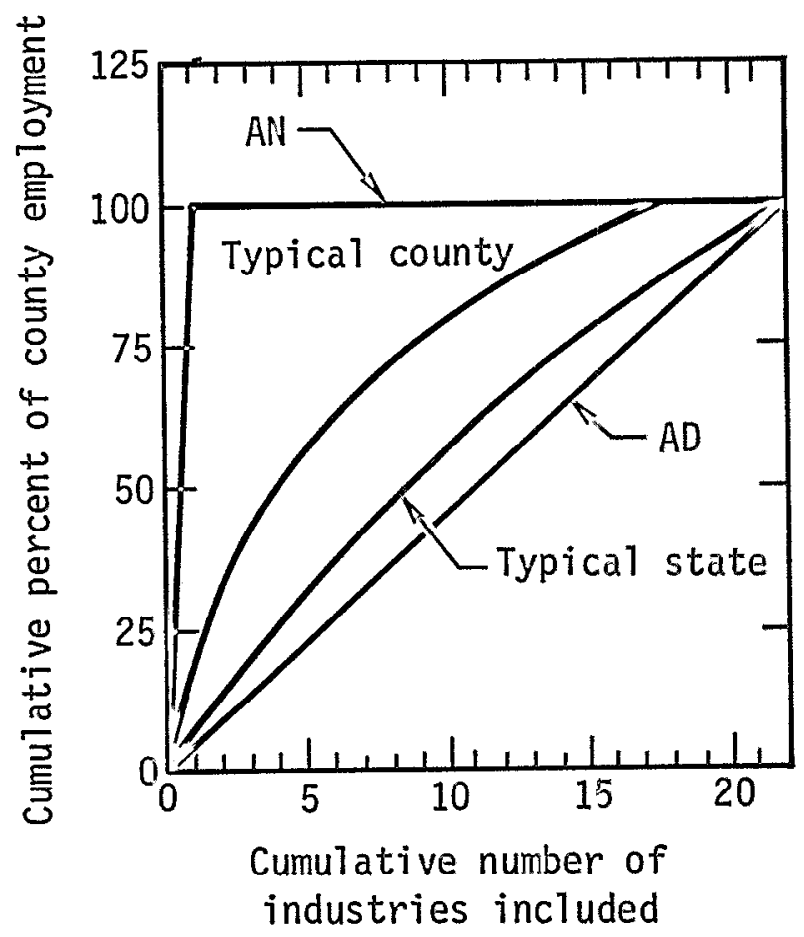

Fig. 6.2. Typical state and county diversity graphs. AN = crude inde: of absolute nondiversification; $\mathrm{AD}=$ crude index of absolute diversification. 


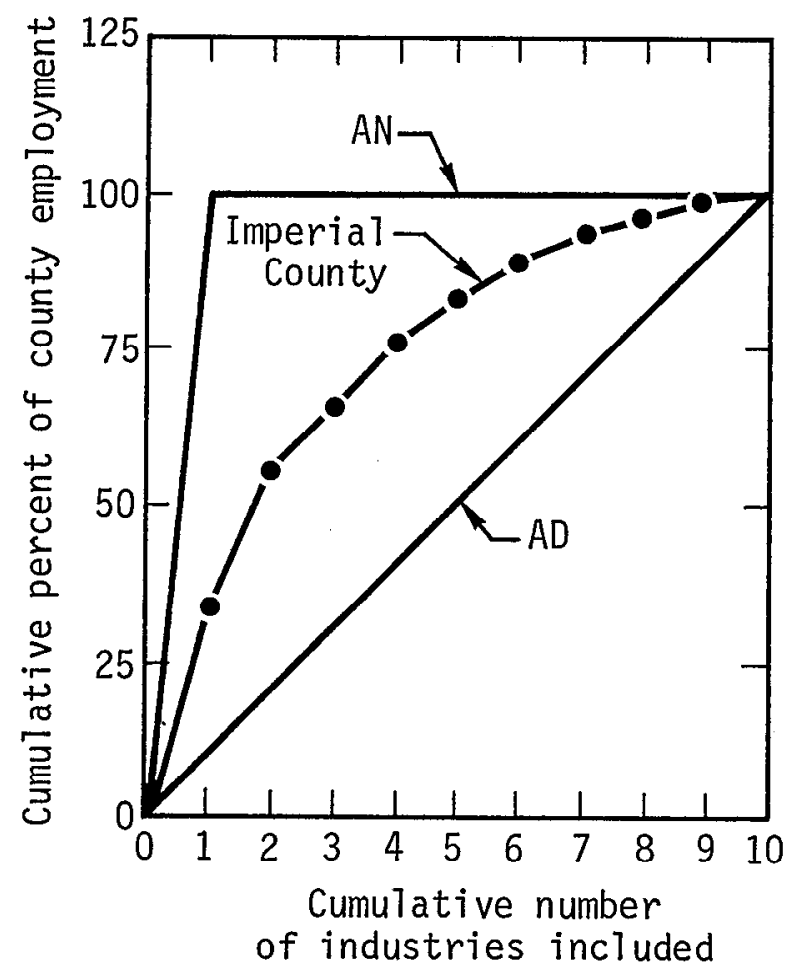

\begin{tabular}{clc}
$\begin{array}{c}\text { Industry } \\
\text { rank }\end{array}$ & \multicolumn{1}{c}{ Industry name } & $\begin{array}{c}\% \text { of County } \\
\text { employment }\end{array}$ \\
1 & All agriculture & 33.41 \\
2 & Retail & 22.40 \\
3 & Services & 10.01 \\
4 & Transportation, communication, & 9.97 \\
& public utility & 7.20 \\
5 & Manufacturing & 6.95 \\
6 & Wholesale & 3.33 \\
7 & Agricultural services & 3.05 \\
8 & Finance, insurance, and & 3.03 \\
9 & real estate & 0.08 \\
10 & Contract construction & Mining
\end{tabular}

Fig. 6.3. Imperial County diversification graph. $A N=$ crude index of absolute nondiversification; $\mathrm{AD}=$ crude index of absolute diversification.

in wholesale sectors, and substantially above average in utilities and agriculturally related areas. The extremely high location quotients for individual sectors associated with public utilities functions (SIC \#'s 4000, 4900, 4931, 4950 and 4970) in Imperial
County should be explained. In general, employment associated with utility companies structured similarly to IID is not recorded on County Business Patterns data. IID data were obtained from Lofting's employment figures ${ }^{2}$ and were included with the County Business Patterns ${ }^{1}$ data for these calculations to better represent total Imperial County employment. However, data were not available to make similar inclusions for other counties. Thus Imperial County appears artificially strong in these areas. Two of these sectors, 4900 and 4970, appear on Table 6.19 but should be disregarded because of this artificial bias.

The general coefficients of localization for the three agricultural divisions are well within the normal range for primary activities. Crop related agriculture shows less diversity than does beef, cattle, and livestock. This difference, however, is too small to be of any real significance.

In Table 6.18, Imperial County ranks 26 th for overal1 county diversity with an RID of 0.5380 , while it ranks 32nd for population, and 31 st for total employment.

\subsection{SUBCOUNTY ECONOMIC ANALYSIS}

\section{Agriculture}

Having described the economy of Imperial County as a whole in previous 
Table 6.15. County economic location quotients by sector.

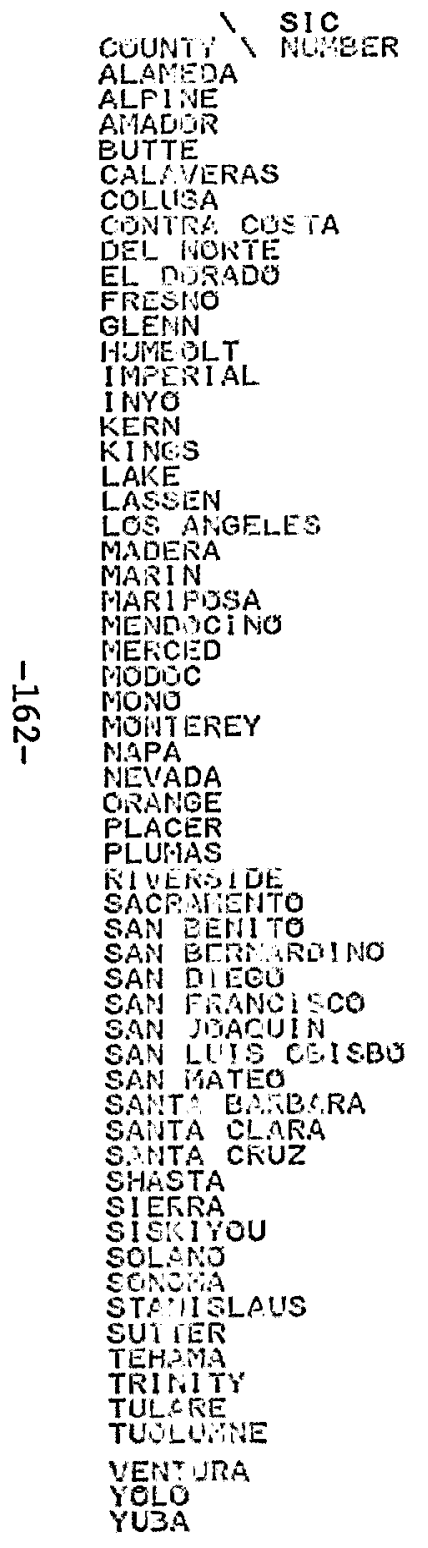

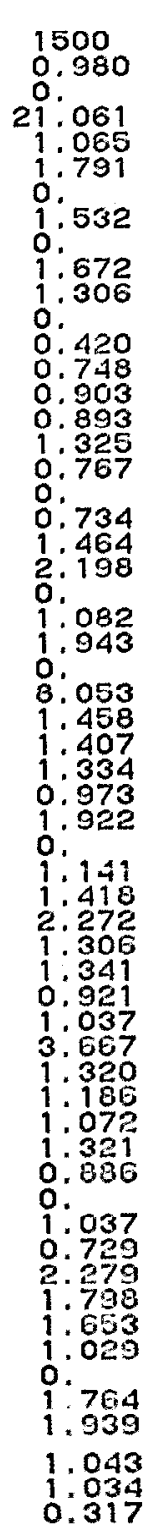

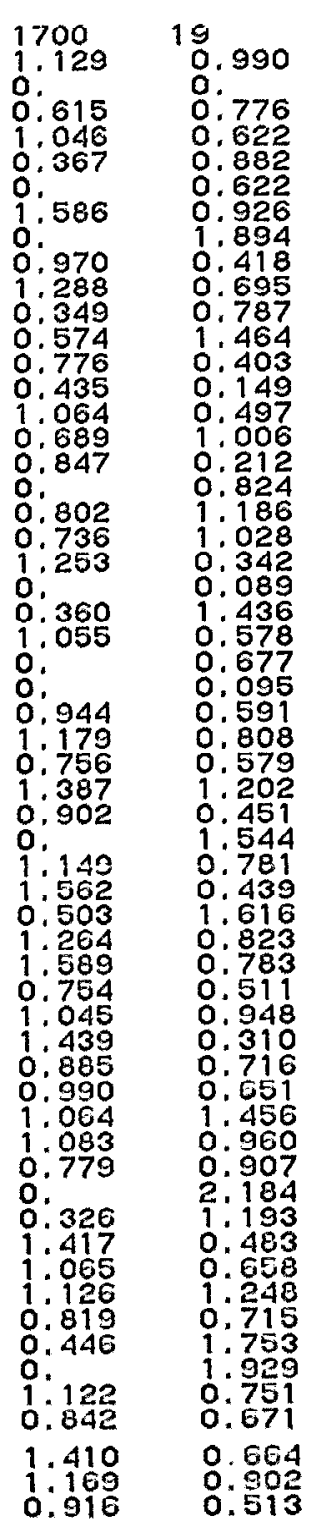

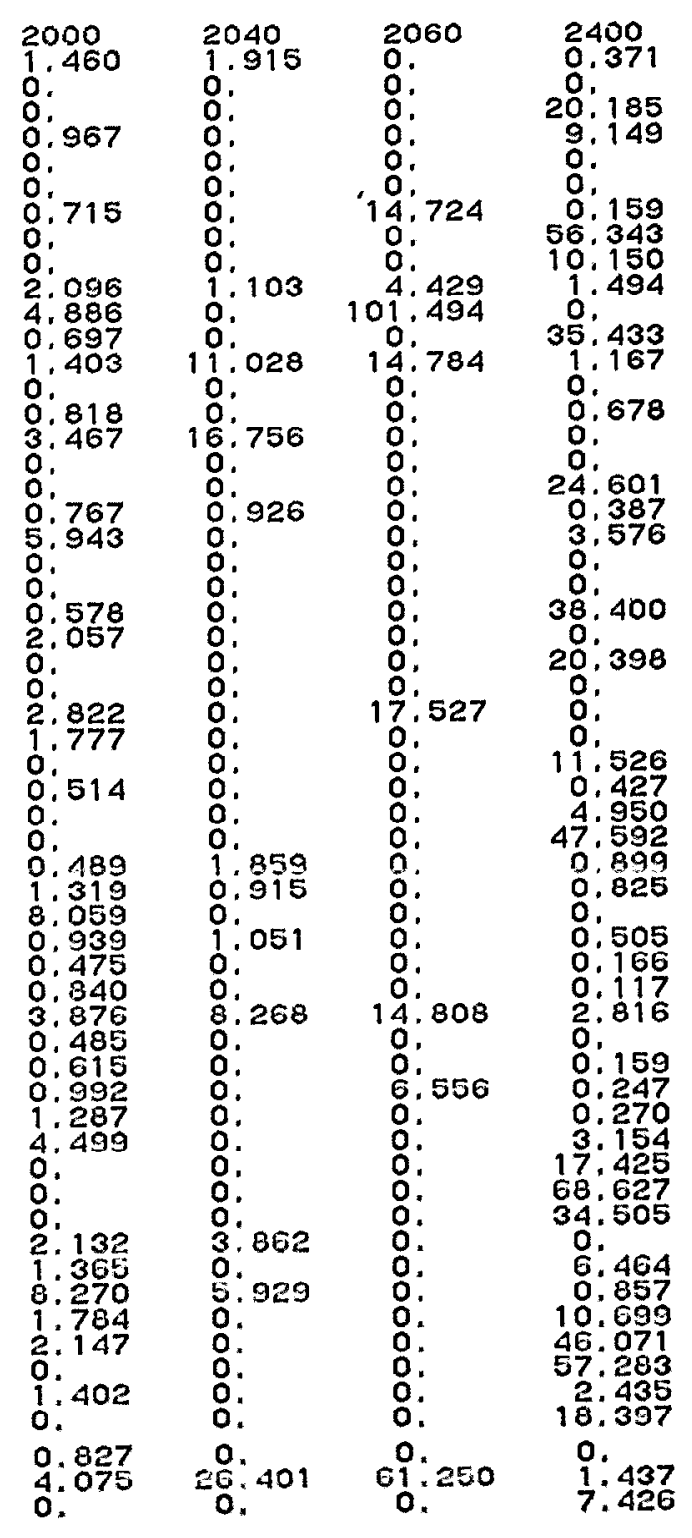


Table 6.15. (Continued)

\begin{tabular}{|c|c|c|c|c|c|c|c|c|c|c|}
\hline 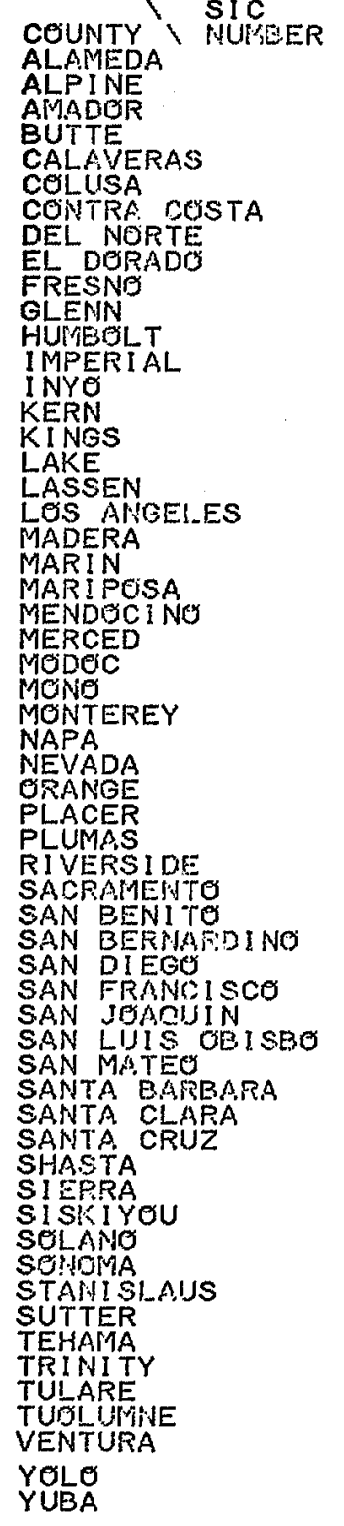 & 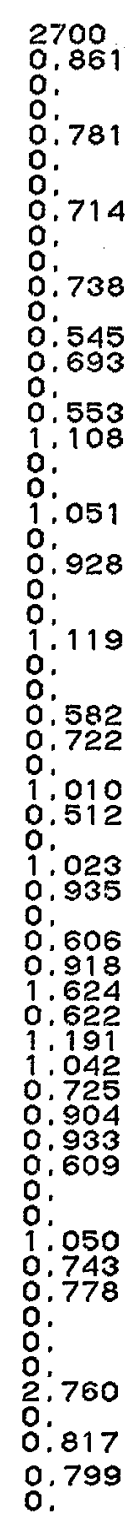 & 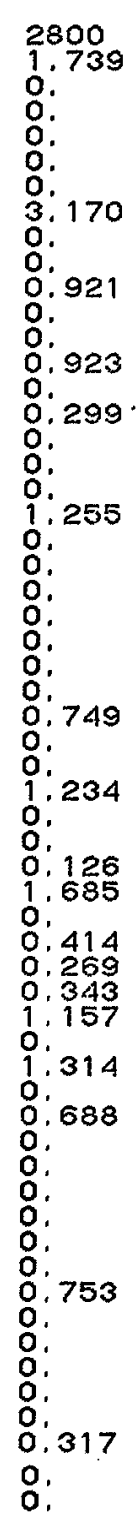 & 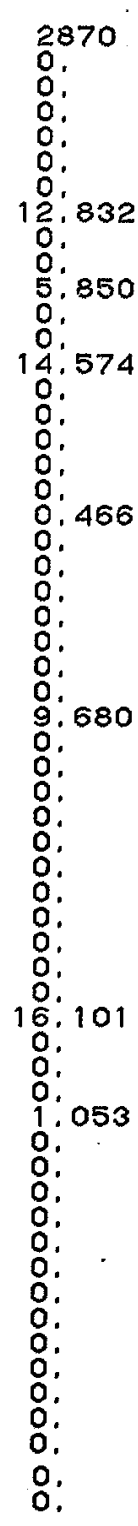 & 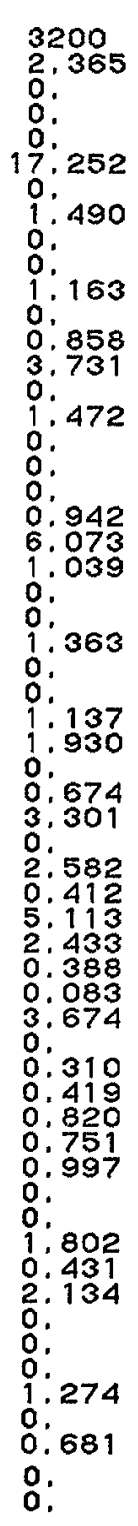 & 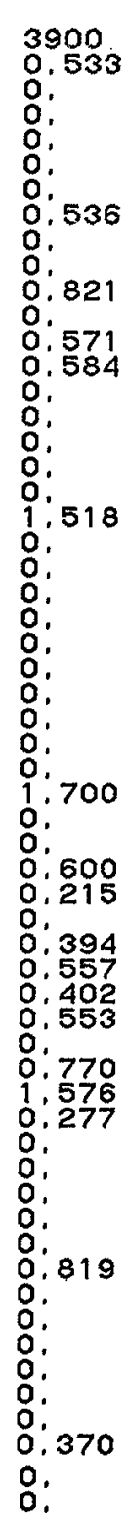 & 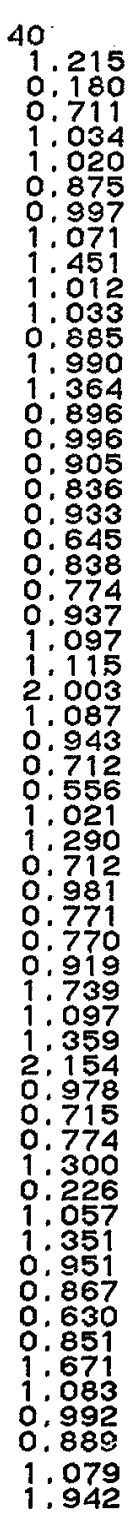 & 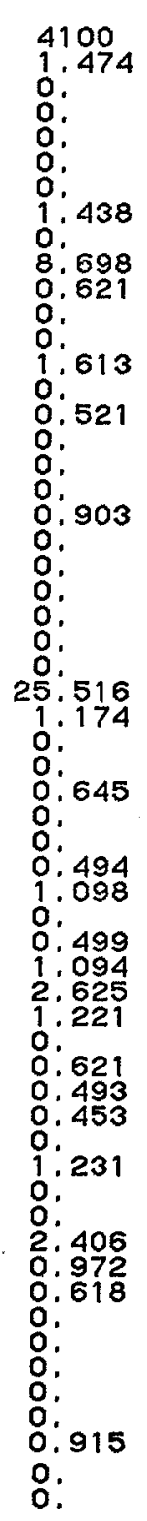 & 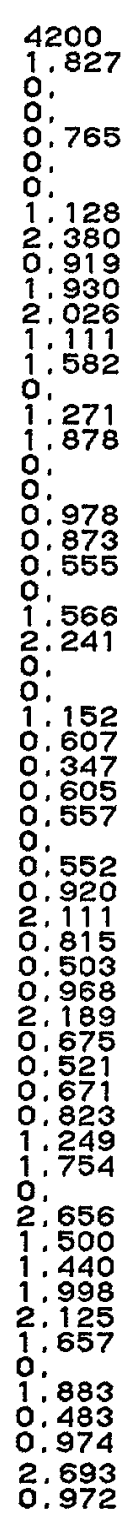 & 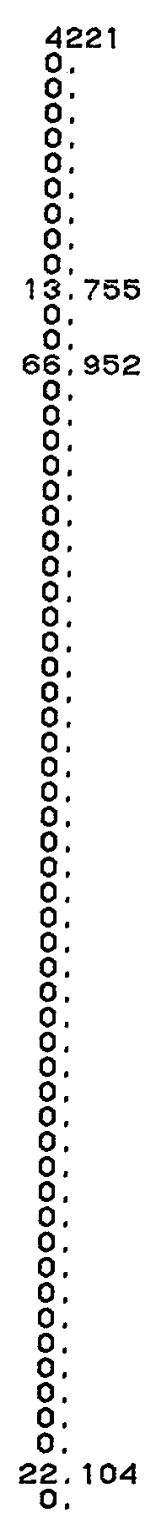 & 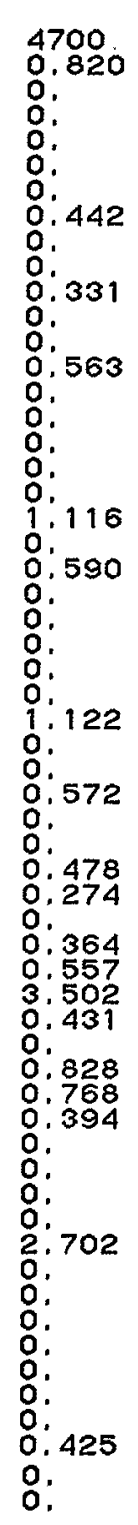 \\
\hline
\end{tabular}


Table 6.15. (Continued)

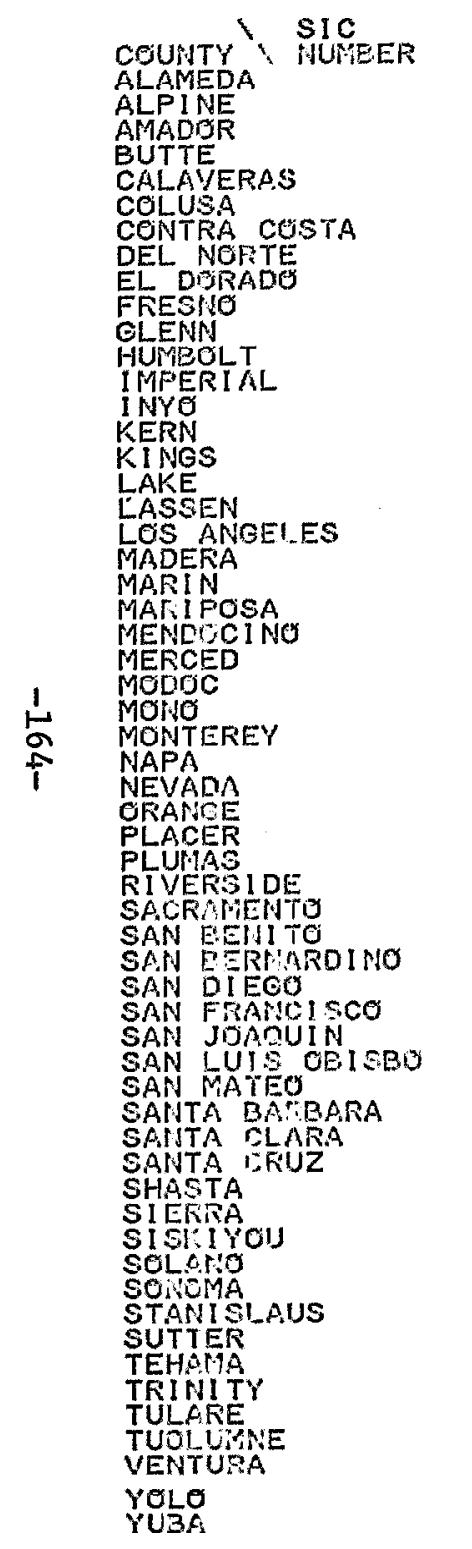

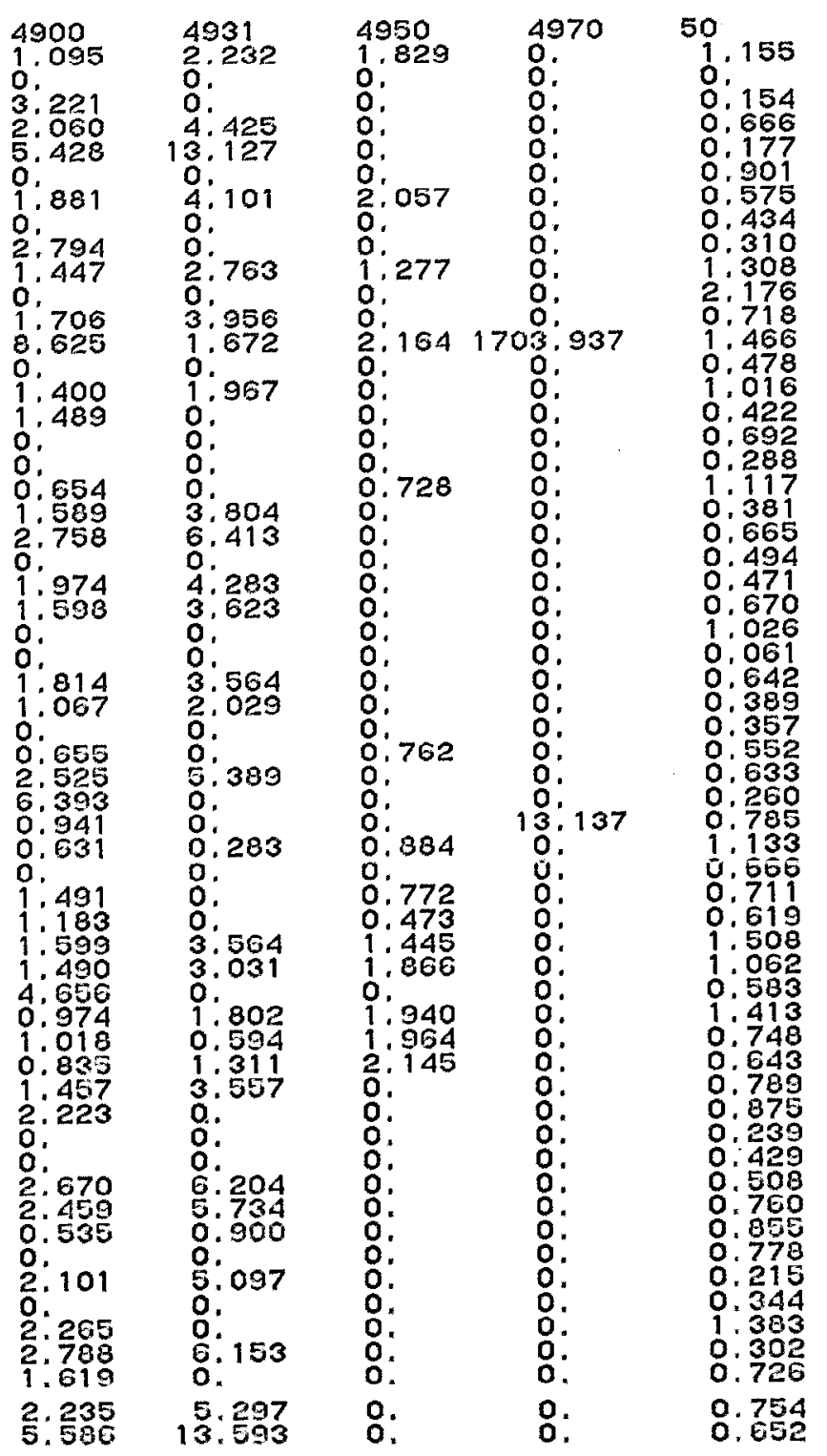

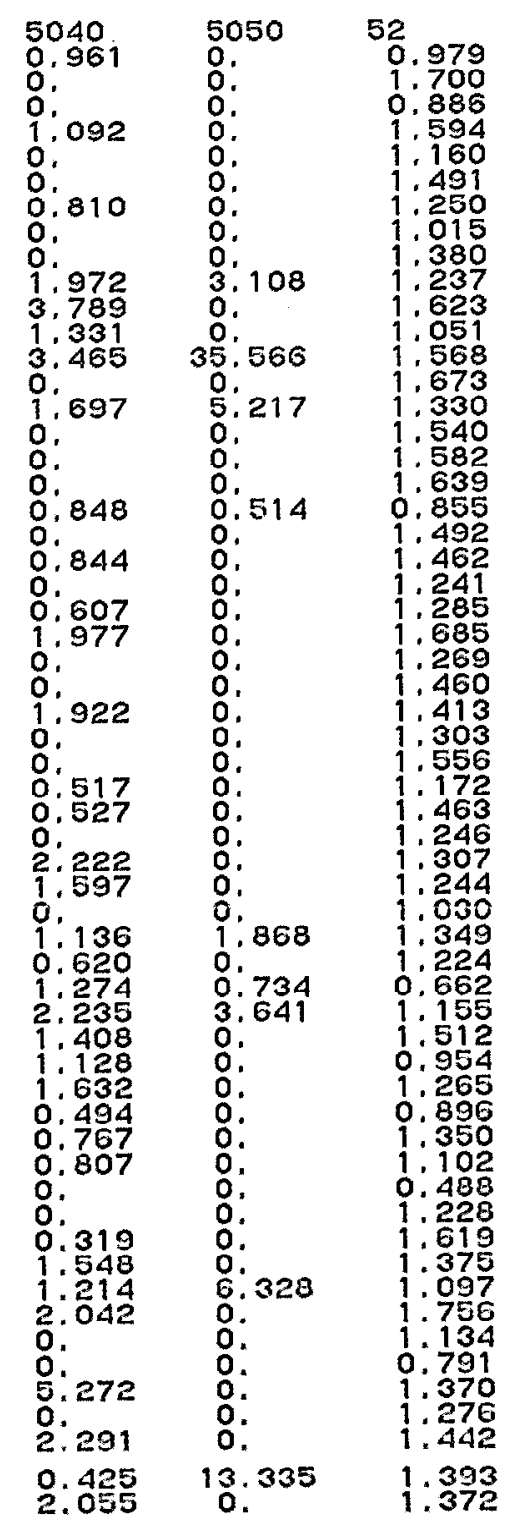

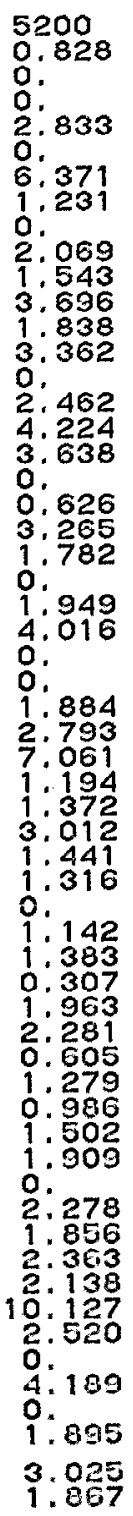

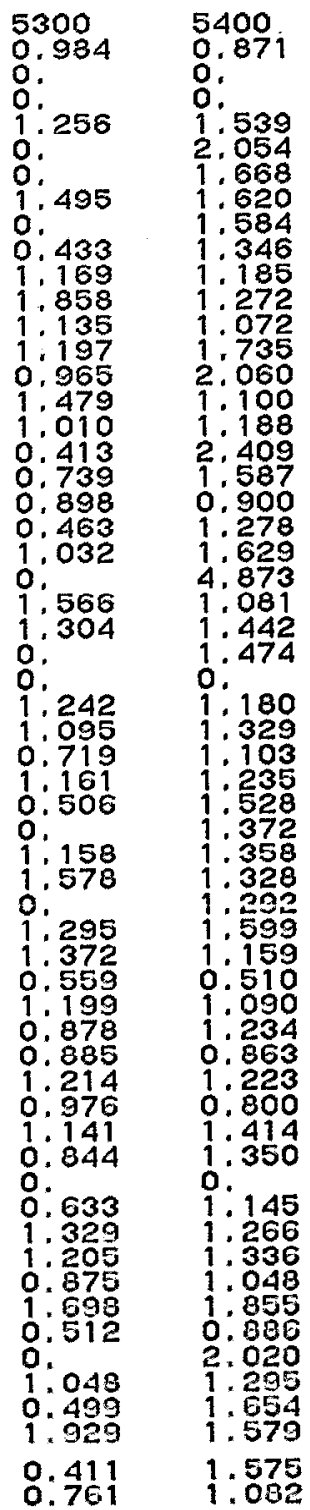


Table 6.15. (Continued)

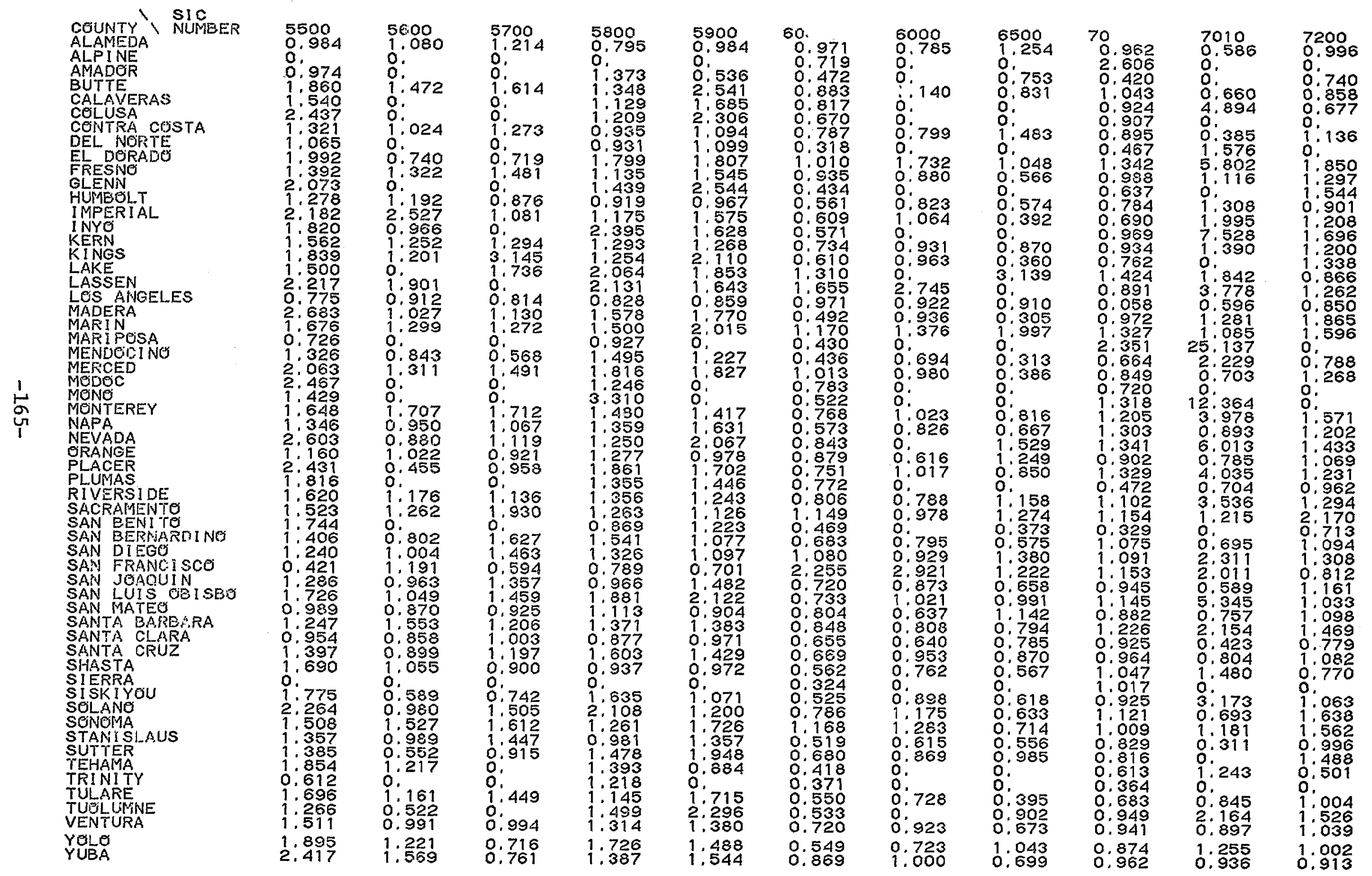


Table 6.15. (Concluded)

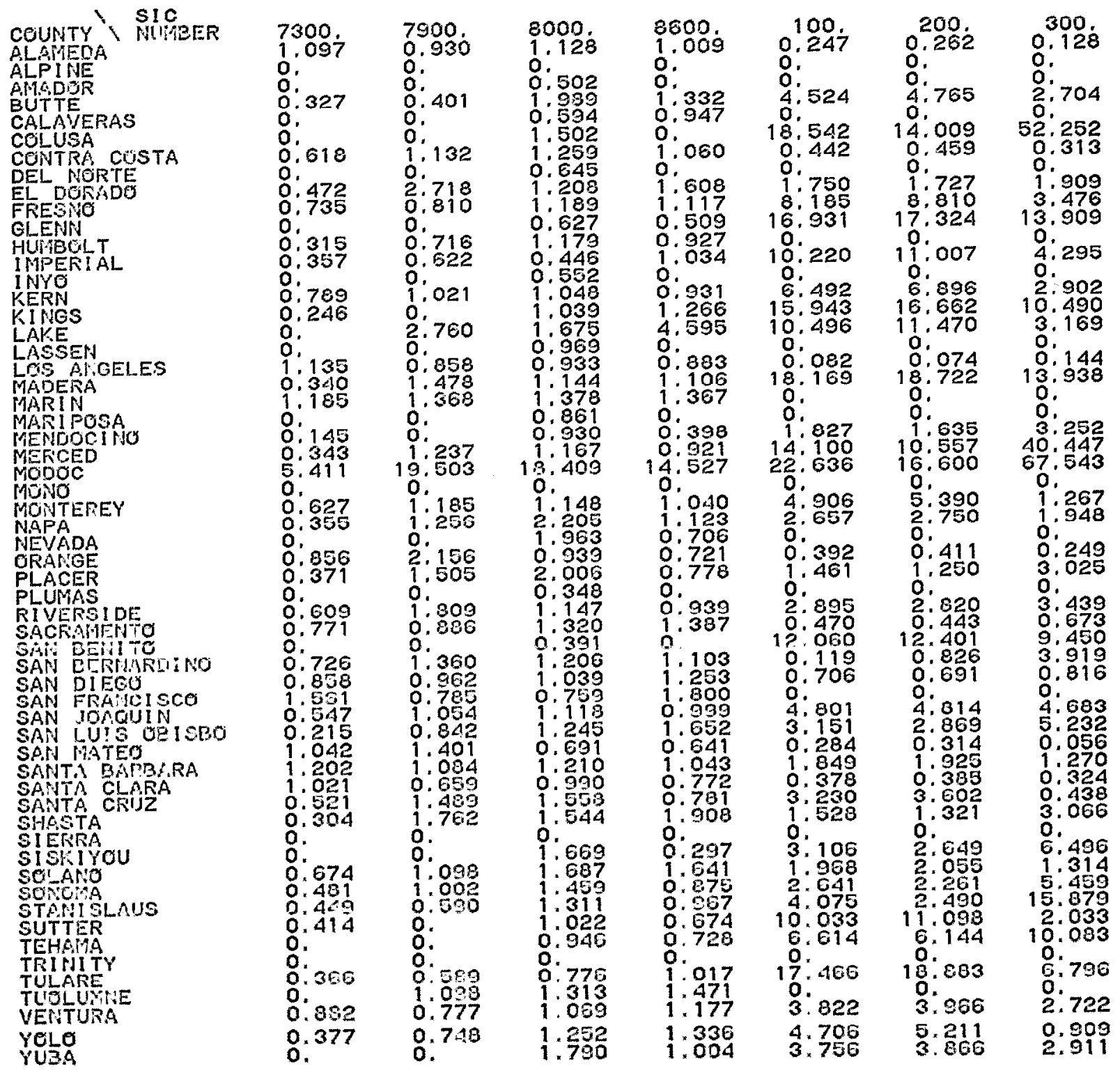


Table 6.16. Coefficients of localization for California agricultural activities in 1972.

All agricultural activities

0.6621

All crop related agriculture

0.6704

Beef cattle and livestock

0.6581

Table 6.17. California County indices of diversification for 1972 .

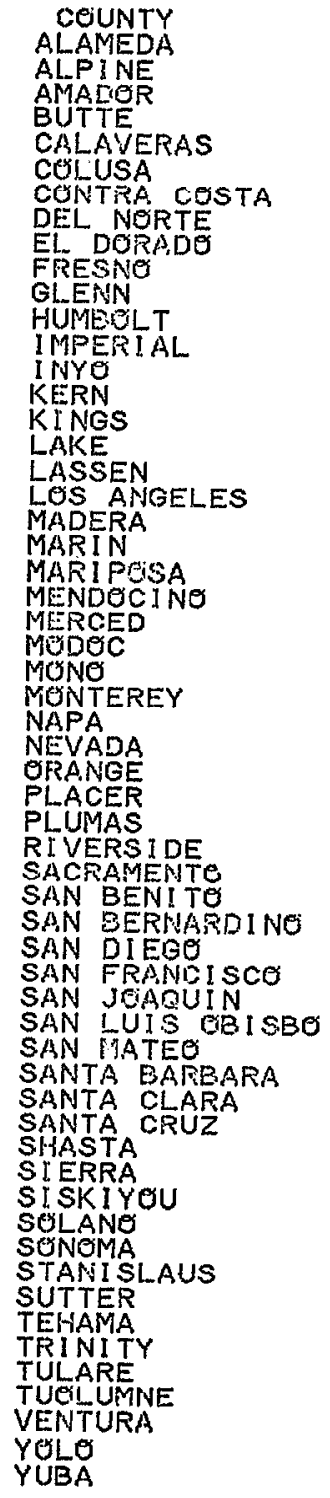

RI D

D. 8859

D. 6329

0.5224

0.5470

0. 5222

0.7274

0.5158

0. 5051

D. 6556

Q. 5380

0.6228

D. 6640

0.6062

ค. 6641

0.6920

0. 5829

0.7158

0. 6064

0.6630

0.6498

0.5019

0.6333

D. 5971

0.5196
0.6966

0. 4896

D. 4587

อ. 6113

0. 5742

0. 4352

0. 4711

0.4977

0. 4730

D. 6248

0.5167

0.4835

0.8118

0. 5533

D. 5344

0. 4722

0.5259

0.6616

D. 6936

0.6206

0.5309

0. 4479

0.5003
0.4652 
Table 6.18. SIC numbers and associated industry titles for Imperial County characterization. 1

\begin{tabular}{|c|c|c|c|c|c|}
\hline $\begin{array}{l}\text { SIC } \\
\text { no. }\end{array}$ & $\begin{array}{l}\text { Digit } \\
\text { level }\end{array}$ & Industry title & $\begin{array}{l}\text { SIC } \\
\text { no. }\end{array}$ & $\begin{array}{l}\text { Digit } \\
\text { level }\end{array}$ & Industry title \\
\hline 07 & 1 & $\begin{array}{l}\text { Agricultural service, } \\
\text { forestry and fishing }\end{array}$ & 2090 & 3 & $\begin{array}{l}\text { Miscellaneous food } \\
\text { products }\end{array}$ \\
\hline 0700 & $2^{a}$ & $\begin{array}{l}\text { Agricultural services } \\
\text { and hunting }\end{array}$ & $\begin{array}{l}2200 \\
2400\end{array}$ & $\begin{array}{l}2 \\
2\end{array}$ & $\begin{array}{l}\text { Textile mill product: } \\
\text { Lumber and nood }\end{array}$ \\
\hline 0800 & 2 & Forestry & 2500 & 2 & Furniture and firtures \\
\hline 0900 & 2 & Fishing & 2600 & 2 & Paper and allied product \\
\hline 10 & 1 & Mining & 2700 & 2 & Printing and publishing \\
\hline 1000 & 2 & Metal mining & 2800 & 2 & Chemical and allied \\
\hline 1300 & 2 & Oil and gas extraction & 2870 & 3 & Agricultural fertilizor \\
\hline 1381 & 4 & Drilling wells & 2900 & 2 & Petroleum and coal \\
\hline 1382 & 4 & Exploration services & 3000 & 2 & Rubber and plastics \\
\hline 15 & 1 & Contract construction & 3200 & 2 & Stone, clay, glass \\
\hline 1500 & 2 & General building & 3300 & 2 & Primary metals \\
\hline 1600 & 2 & Heavy construction & 3400 & 2 & Fabricated metals \\
\hline 1700 & 2 & $\begin{array}{l}\text { Special trade } \\
\text { construction }\end{array}$ & 3520 & 3 & Farn machinery \\
\hline 19 & 1 & Manufacturing & 3530 & 3 & Construction machinery \\
\hline 2000 & 2 & $\begin{array}{l}\text { Food and kindred } \\
\text { products }\end{array}$ & $\begin{array}{l}3600 \\
3700\end{array}$ & 2 & $\begin{array}{l}\text { Electrical machinery } \\
\text { Transportation equipment }\end{array}$ \\
\hline 2010 & 3 & Meat products & 3800 & 2 & Instruments and related \\
\hline 2030 & 3 & $\begin{array}{l}\text { Canned, Cured, and } \\
\text { Erozen products }\end{array}$ & 3900 & 2 & $\begin{array}{l}\text { Miscellaneous } \\
\text { manufacturing }\end{array}$ \\
\hline $\begin{array}{l}2040 \\
2060\end{array}$ & 3 & $\begin{array}{l}\text { Grain mill products } \\
\text { Sugar }\end{array}$ & 40 & 1 & $\begin{array}{l}\text { Transportation and } \\
\text { public utilities }\end{array}$ \\
\hline
\end{tabular}

"Two-digit-level SIC's are subsets of the previous one digit SIC's; three-digit SIC's are subsets of two-digit SIC's and four-digit SIC's are subsets of threcdigit SIC's. 
Table 6.18. (Continued)

\begin{tabular}{|c|c|c|c|c|c|}
\hline $\begin{array}{l}\text { SIC } \\
\text { no. }\end{array}$ & $\begin{array}{l}\text { Digit } \\
\text { leve1 }\end{array}$ & Industry title & $\begin{array}{l}\text { SIC } \\
\text { no. }\end{array}$ & $\begin{array}{l}\text { Digit } \\
\text { leve1 }\end{array}$ & Industry title \\
\hline 4100 & 2 & $\begin{array}{l}\text { Local and interurban } \\
\text { transportation }\end{array}$ & 5400 & 2 & Food \\
\hline 4150 & 3 & School busses & 5500 & 2 & Auto \\
\hline 4200 & 2 & $\begin{array}{l}\text { Trucking and } \\
\text { warehousing }\end{array}$ & 5700 & 2 & $\begin{array}{l}\text { Clothes } \\
\text { Furniture }\end{array}$ \\
\hline 4221 & 4 & $\begin{array}{l}\text { Farm product } \\
\text { warehousing }\end{array}$ & 5800 & 2 & $\begin{array}{l}\text { Eating and drinking } \\
\text { places }\end{array}$ \\
\hline 4500 & 2 & Transport by air & 5900 & 2 & Miscellaneous retail \\
\hline 4600 & 2 & Pipeline transport & 60 & 1 & $\begin{array}{l}\text { Finance, insurance and } \\
\text { real estate }\end{array}$ \\
\hline 4700 & 2 & Transportation service & & & \\
\hline 4800 & 2 & $\begin{array}{l}\text { Communication and } \\
\text { utilities }\end{array}$ & $\begin{array}{l}6000 \\
6500\end{array}$ & 2 & $\begin{array}{l}\text { Banking } \\
\text { Real estate }\end{array}$ \\
\hline 4900 & 2 & $\begin{array}{l}\text { Electricity, gas and } \\
\text { sanitary services }\end{array}$ & 70 & 1 & Services \\
\hline 4931 & 4 & $\begin{array}{l}\text { Electricity and other } \\
\text { services }\end{array}$ & $\begin{array}{l}7010 \\
7030\end{array}$ & $\begin{array}{l}3 \\
3\end{array}$ & $\begin{array}{l}\text { Hotel and motel } \\
\text { Trailer park and } \\
\text { recreational camps }\end{array}$ \\
\hline 4940 & 3 & Water suppIy & 7200 & 2 & Personal services \\
\hline $\begin{array}{l}4950 \\
4970\end{array}$ & 3 & $\begin{array}{l}\text { Sanitary } \\
\text { Irrigation services }\end{array}$ & 7300 & 2 & $\begin{array}{l}\text { Miscellaneous business } \\
\text { services }\end{array}$ \\
\hline 50 & 1 & Wholesale & 7512 & 4 & Car rental and leasing \\
\hline 5010 & 3 & Auto and related & 7800 & 2 & Motion picture \\
\hline 5040 & 3 & Groceries and related & 7900 & 2 & $\begin{array}{l}\text { Amusement and recreational } \\
\text { services }\end{array}$ \\
\hline $\begin{array}{r}5050 \\
52\end{array}$ & 3 & $\begin{array}{l}\text { Farm Products (raw) } \\
\text { Retail }\end{array}$ & 7940 & 3 & $\begin{array}{l}\text { Miscellaneous amusement } \\
\text { and recreational services }\end{array}$ \\
\hline 5200 & 2 & $\begin{array}{l}\text { Building and farm } \\
\text { equipment }\end{array}$ & 8000 & 2 & Medical and health \\
\hline 5300 & 2 & General store & $\begin{array}{l}8060 \\
8200\end{array}$ & $\begin{array}{l}3 \\
2\end{array}$ & $\begin{array}{l}\text { Hospitals } \\
\text { Education services }\end{array}$ \\
\hline
\end{tabular}


Table 6.18. (Concluded)

\begin{tabular}{lccccc}
\hline $\begin{array}{l}\text { SIC } \\
\text { no. }\end{array}$ & $\begin{array}{l}\text { Digit } \\
\text { level }\end{array}$ & Industry title & $\begin{array}{l}\text { SIC } \\
\text { no. }\end{array}$ & $\begin{array}{l}\text { Digit } \\
\text { level }\end{array}$ & Industry title \\
\hline 8600 & 2 & Nonprofit organization & $200^{\mathrm{b}}$ & 3 & Crop related agrlculturc \\
$100^{\mathrm{b}}$ & 2 & All agriculture & $300^{\mathrm{b}}$ & Beef cattle and 1ivestocl:
\end{tabular}

${ }^{b}$ Data not from County Business Pattern SIC information. Provided by Dr. Loftinf.

Table 6.19. Imperial County sectors of major state importance.

\begin{tabular}{rlr}
\hline $\begin{array}{c}\text { SIC } \\
\text { number }\end{array}$ & \multicolumn{1}{c}{ Sector name } & Location quotient \\
\hline 4970 & Irrigation services & 1703.937 \\
4221 & Farm product warehousing & 66.952 \\
5050 & Farm product (raw) wholesaling & 35.566 \\
2060 & Sugar manufacturing & 14.784 \\
2870 & Agricultural fertilizer manufacturing & 14.574 \\
2040 & Grain mill products & 11.028 \\
200 & Crop related agriculture & 11.007 \\
100 & All agriculture & 10.220 \\
700 & Agricultural support & 10.174 \\
4900 & Electricity, gas, and sanitary services & 8.625 \\
300 & Beef, cattle, and livestock & 4.295 \\
3200 & Stone, clay and glass manufacturing & 3.731 \\
5040 & Groceries and related wholesale & 3.465 \\
5200 & Building and farm equipment, wholesale & 3.362 \\
\hline
\end{tabular}


Table 6.20. Comparison of selected sector location quotients for Kern, Fresno, and Imperial Counties.

\begin{tabular}{rrrr}
\multicolumn{1}{c}{ SIC } \\
number & Imperia1 County & Fresno County & Kern County \\
\hline 4970 & 1703.937 & 0 & 0 \\
4221 & 66.952 & 13.755 & 0 \\
5050 & 35.566 & 3.108 & 5.217 \\
2060 & 14.784 & 4.429 & 0 \\
2870 & 14.564 & 5.850 & 0 \\
2040 & 11.028 & 1.103 & 16.756 \\
200 & 11.007 & 8.810 & 6.896 \\
100 & 10.220 & 8.185 & 6.492 \\
700 & 10.174 & 1.818 & 2.242 \\
4900 & 8.625 & 1.447 & 1.400 \\
300 & 4.295 & 3.476 & 2.902 \\
3200 & 3.731 & 1.163 & 1.472 \\
5040 & 3.465 & 3.789 & 1.697 \\
5200 & 3.362 & 3.696 & 2.462 \\
& & & \\
\hline & & & \\
\hline
\end{tabular}

sections, we will now provide a cursory description of the spatial variations of economic activity within the county. Of particular interest to this study is the distribution of activity with respect to the four principal known Geothermal Resource Areas (KGRAs) in the Imperial Valley. Figure 6.4 shows the location of Imperial Valley's 67 active and inactive feed lots mapped against KGRA boundaries. If future county ordinances for geothermal powerplant siting were to require at least a $1-m i$ separation between a powerplant and feed lot, each feed lot located within a KGRA will eliminate almost $5 \mathrm{mi}^{2}$ (3100 acres) of potential area for plant siting.

Sector Intracounty Distribution Industry. Most of the county's industry is centered in slightly over 700 acres of industrially zoned land in the city of El Centro, along the short axis between E1 Centro and Imperial and particularly the area around the commercial airport and county fair grounds. A secondary center exists in Calexico. Minimal industrial activity 


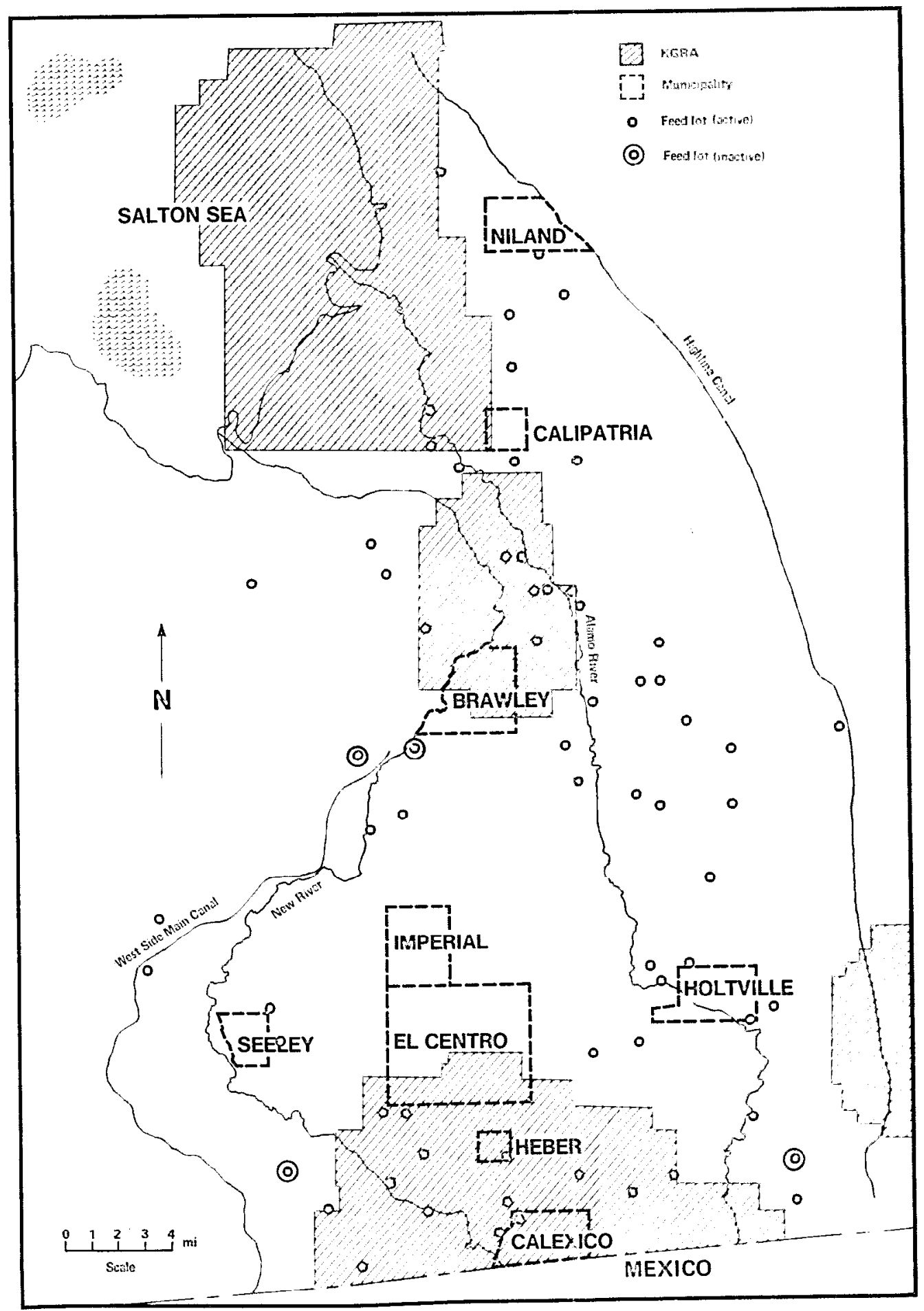

Fig. 6.4. Imperial Valley feed lot operations mapped against KGRAs. 
exists in Imperial Valley outside these areas. County land use plans for the future call for development of industrial centers north of Seeley in the Mesquite Lake area and just east of Niland (see subsection 7.4). In addition to the present spatial concentration of industrial activity, several individual sectors for the county are concentrated in one or two major activities (i.e. agricu1tural fertilizers, sugar production, federal government, Anza meat packers, U.S. Gypsum, stone and clay manufacturing, mining, etc.).

Commerce. This category includes wholesale, reta11, and service sectors. Again, these activities, especially the more specialized individual activities, are centered in $\mathrm{E} 1$ Centro. Commercial activities are, however, slightly more diversified than industrial. Brawley serves as a general retail center for the northern end of the Valley, and Calexico with one of the highest retail sales to population ratios for a California city is an extensive and rapidly growing retail center serving both the southern sections of the Imperial Valley and Mexicali. In 1974, E1 Centro recorded $51.6 \%$ of the county retail sales (see Table 6.21) while Calexico recorded $37.9 \%$. Brawley was a distant third with over $7 \%$ of the total county retail sales.
Recreation. Primary recreational activities within Imperial County are centered on sand dune related activities (especially in The Dunes Recreational Area) and principally on dune buggy activities. Secondary to this class of recreation is water related activities. Water related recreation within the county is fairly evenly split between the Colorado River and the Salton Sea based on the number of permanently moored vessels, available moorings, estimated recreator days, and on county staff estimates. Several small freshwater lakes along the New and Alamo Rivers comprise the rest of the county water related activity.

Estimates of the total annual recreational value of the Salton Sea have been set at $\$ 11,000,000$ by the U.S. Department of the Interior. ${ }^{21}$ Previous estimates of Salton Sea recreational use and economic value for 1967 made by the State Department of Fish and Game ${ }^{22}$ are shown in Table 6.21. As of 1970, Arnett, director of the California Department of Fish and Game, estimated that the 1967 use figure approximated capacity use levels. In both the 1973 U.S. Department of Interior and the California Department of Fish and Game studies, the two sites on the Salton Sea receiving heaviest recreational use were in Riverside County. This dominance of Riverside County over 
Imperial County in recreational

use of the Salton Sea may be a par-

tial explanation of the view

expressed to us during interviews

held with numerous Imperial County

staff officers on March 9 and 10,

1976, that Imperial County does not

consider the salton sea as a major

recreational or a significant

economic resource.
E1 Centro Economy

Data have been gathered on aspects of the E1 Centro city conomy; some of these statistics are 1isted in Table 6.22. Comparison of this data to county data shows the dominance of El Centro within the county economy and general grorth pittern: of both the city and county over thu past 5 years.

Table 6.21. Recreational use of the Salton Sea and resultant economic benefit in 1967.22

\begin{tabular}{|c|c|c|}
\hline Activity & Recreation days & $\begin{array}{l}\text { Total } \\
\text { economic benefit, } \$\end{array}$ \\
\hline Fishing & 356,000 & 719,200 \\
\hline Hunting & 42,000 & 103,000 \\
\hline General recreation ${ }^{a}$ & $1,100,100$ & $1,200,300$ \\
\hline Tota1 & $1,498,100$ & $2,022,500$ \\
\hline
\end{tabular}

${ }^{a}$ Includes boating, swimming, water skiing, camping, and picnicling. 
Table 6.22. El Centro: Indicators of economic activity. ${ }^{a}$

Persons employed

El Centro total

$\%$ County total

Manufacturing

Government (city, county)

Miscellaneous economic indicators

Building permits

Bank deposits

Total retail sales

Automobile registrations
1326

1067

1970

$$
2,627,476
$$

$\$ 95,746,424$

$\$ 67,769,000$

55,033
1972

$$
\begin{array}{r}
5,332,417 \\
\$ 143,743,034 \\
\$ 79,422,000 \\
59,890
\end{array}
$$

89.2

38.18

\section{4}

$3,126,544$

$\$ 152,737,345$

$\$ 100,706,000$

65,672

${ }^{a}$ From E1 Centro Chamber of Commerce (1974). ${ }^{20}$ 


\section{REFERENCES}

1. U.S. Department of Commerce, Bureau of the Census, 1972 County Busincse Patterns for California Counties.

2. E.M. Lofting, Economy Modelling Study of Imperial County, Califomia Dryland Research Institute, University of California, Riverside, Preliminary Rept. (1976).

3. Imperial County Planning Department, Imperial County ULtimate Land Use PZan, June 25, 1973.

4 G.F. Snow, "An Overview of Salton Sea Agriculture," Compendium of Fapors Imperial Valley - Salton Sea Area Geothemol Hearing, California State Resources Agency, Sacramento, California, dictober 1970.

5. Imperial Valley Development Agency, Standard Inäustrial Survey Summaty Report, Imperial County, California, September 1969.

6. California Crop and Livestock Reporting Service, California Vegetable Cropo 1964-1972, Dec. 1973.

7. Imperial Irrigation District, Annual Inveniony of Areas Receiving Diatrict Water for $1973,1974,1975$.

8. Imperial County, office of the Agriculturai Comissioner, Imcenial County Agriculture, 1972.

9. Imperial County, Office of the Agricultural Commissioner, Imperial County Agriculture, 1974.

10. H.E. Albanez, Imperial Valley Agricultural Labor Representative, private communication (December 10, 1975).

11. A.F. Van Maren, Director Imperial County Arricultural Extension Service, Gudielines to Production Costs and Fractices, 1975-76, Imoerial County Crops, Circular 104 University of California Agricultural Extension Service, Sept. 1975.

12. Office of Southern California Employment Data and Research. Area Manpower Review, Imperial County, 1976-1977. Jan. 1976.

13. Office of Southern California Employment Data and Research. "Tota1 Civilian Labor Force, Unemployment, Rate by Place of Residency, Non Ag Vage and Salary Employment by Place of Jobs. Imperial County 1975." Unpublished. 
14. U.S. Department of Commerce, Bureau of the Census, 1970 Census Survey for California Counties.

15. California Employment Development Department. Imperial County Labor Market Buzzetin, Jan-Mar 1976.

16. Renaldo, P., Imperial County Social-Economic Characterization. Unpublished Data Report for Campesinos Unidos, February 1976.

17. H.E. Albanez, "Imperial County Pre-season Farm Labor Reports, 1976," unpublished data supplied to the office of Southern California Data and Research, Dec. 1975.

18. Imperial Irrigation District. Annual Report to the Federal Power Commission. for FY 1972, 1974 and 1975.

19. W. Isard, Methods of Regional Analysis, The MIT Press, Cambridge Mass., 1960.

20. E1 Centro Chamber of Commerce, Community Economic Profize for EZ Centro, Imperial County, California, August 1974.

21. U.S. Department of the Interior, Final Environmental Statement for the Geothermal Leasing Progrom, Volume II, 1973.

22. G.R. Arnett, "Recreational Implication of the Salton Sea Area Geothermal Resource Development," Compedium of Papers, Imperial ValZey-SaZton Sea Area Geothermal Heariny, State of California Resources Agency, Sacramento, California, October 1970. 


\section{Section 7 \\ County Fiscal Characterization}

Kenda11 Haven

\subsection{FISCAL BUDGET ANALYSIS}

This basic county fiscal

characterization is based on budget

items as listed in the 1975-1976

County Final Eudget, Schedule 5,

Adopted Expenditures, 1 and on 1974

estimated county populations. The

Trmpexia1 County budget and per

capita county budget are shown in

Table 7.1. Comparisons ath budgets for other selected counties for 1975-1976 are shom in Tables 7.2 and

7.3. Table 7.2 shows location quotients for each budget itom and Table 7.3 shors budget items as a percentage of total county budgets. State budget and budget item totals for like calculation of location quotients vere not taken from the state budget. State and county budgetary functions are very different; thece is no direct relationship between county and state expenditure. Rather, state totals as used here represent the sum of all county budgets and budget items. Finally, per capita budgecs for selected counties are listed in Table 7.4, and comparalive location quotients For each per capita budget item are shown in Table 7.5. Zero budget item entrics such as ite protection or debt service indicate that no money was appropriated under that given line item title. Comp. ing the data in Tables 7.2 and 7.3 (toral budget lata) with that in Tables 7.4 and 7.5 (per capita budget d.tici) gives a relatively complete picturc of Imperial County fiscal spendins patterns.

Based on the total budget datat of Tablas 7.2 and 7.3 and discerard tor: plant aequisition, Imperial County appears to be fairly typical in its relativa fiscal spending habits with the exceptions of health and sanitation and public issistanca. Relative Imperial County expunsus are substantially belor the 10-county norm for both health and public assistance. However, per capita figures indicate Imperial County $i s$ very near the state normative valut for public assistance, but is alihtly below average for health. Sanitation spending, the other subcategory undor health and sanitation, is unusully high for Imperial County and, at $\$ 5.69$ per person, is far Larger than for any other councy on a per capita basis. Imperial County also spont eignifiadnty more in fiscal year 1975-1976 for public naye thrin roy other count: analyzed an a pre cuptit basis. Horever this budget caterory 
Table 7.1. Imperial County budget for 1975-76. ${ }^{1}$

Total Budget

Genera1 government

Plant acquisition

Public protection

Courts

Police

Fire

Health and sanitation

Health

Sanitation

Public assistance

Education

Public ways

Recreation

Debt

Reserve and contingency

General government less

plant acquisition
$\$ 38,722,551$

$11,422,185$

$7,482,968$

$7,861,455$

$1,652,959$

$1,715,332$

746,850

$2,532,347$

$2,112,101$

420,246

$11,641,922$

410,064

$3,742,385$

313,438

0

780,755

$3,939,217$
$\$ 519.82$

153.33

100.45

105.53

22.19

23.03

10.03

34.00

28.35

5.64

156.28

5.15

50.24

4.45

0.00

10.48 is related as much to area as it is to population, and its high value reflects the extensive county road system in the Imperial Valley. As mentioned above, per capita Imperial County public assistance expenditure is not low. If Mexican green card labor acted as an effective buffer for county employment and unemployment, certain portions of county spending in this category would be expected to be low for Imperial county.
County revenues for FY 1975-1976 are listed in Table 7.6. The relative distribution of these revenues is assumed representative of future fiscal years, and these revenue percentages may, on this basis, be used for future analytical purposes. Almost $50 \%$ of county revenue comes from state and federal agencies. of the county generated funds, $37 \%$ came from current property tax and $35 \%$ from carryover funds. Of the 
Table 7.2. Comparative county budget location quotients for selected budget categories.

\begin{tabular}{|c|c|c|c|c|c|c|c|c|c|c|c|}
\hline $\begin{array}{l}\text { COUNIT } \\
\text { CONTRA COSTA } \\
\text { FRESINO } \\
\text { I MFERIAL } \\
\text { KERN } \\
\text { NOS AIGELES } \\
\text { NAPA RIVEIDE } \\
\text { SACRALIENTO } \\
\text { SAI DIIESO } \\
\text { SAN MATEO }\end{array}$ & $\begin{array}{r}\text { GEN } \\
\text { GOVT. } \\
0.408 \\
0.797 \\
1.418 \\
0.610 \\
1.087 \\
0.917 \\
0.905 \\
0.626 \\
1.254 \\
0.715\end{array}$ & $\begin{array}{l}\text { (PLANT } \\
\text { ACN1I) } \\
0.369 \\
2.804 \\
8.258 \\
2.373 \\
0.428 \\
2.850 \\
1.295 \\
0.937 \\
2.207 \\
3.323\end{array}$ & $\begin{array}{l}\text { PUBLIC } \\
\text { PRETECT } \\
1.145 \\
1.054 \\
1.255 \\
1.549 \\
0.865 \\
1.255 \\
1.355 \\
1.320 \\
1.100 \\
1.404\end{array}$ & $\begin{array}{l}\text { (COURTS) } \\
1.179 \\
1.035 \\
0.964 \\
0.816 \\
0.907 \\
0.834 \\
1.007 \\
1.398 \\
1.289 \\
1.228\end{array}$ & $\begin{array}{l}\text { (FOLICE) } \\
0.996 \\
1: 102 \\
1: 035 \\
1: 301 \\
0: 924 \\
1: 243 \\
1: 487 \\
1: 472 \\
1: 149 \\
0.285\end{array}$ & $\begin{array}{l}\text { (FIRE) } \\
\circ: \\
0: \\
2.029 \\
8.860 \\
0.900 \\
2: 280 \\
1.404 \\
0: \\
0: 506\end{array}$ & $\begin{array}{c}\text { HEALTH + } \\
\text { SAN } \\
1.102 \\
0.586 \\
0.444 \\
1.088 \\
1.101 \\
0.581 \\
0.534 \\
0.981 \\
0.518 \\
1.355\end{array}$ & $\begin{array}{l}\text { (HEALTH) } \\
1.123 \\
0.596 \\
0.382 \\
1.085 \\
1.110 \\
0.591 \\
0.546 \\
1.012 \\
0.433 \\
1.393\end{array}$ & $\begin{array}{l}(S .11) \\
0.443 \\
0.278 \\
2.384 \\
1.171 \\
0.820 \\
0.127 \\
1.780 \\
0.018 \\
3.184 \\
0.164\end{array}$ & $\begin{array}{l}\text { PUBLIC } \\
\text { ASS1.ST } \\
1.148 \\
1.134 \\
0.776 \\
0.822 \\
0.995 \\
0.066 \\
1.069 \\
1.013 \\
1.035 \\
0.850\end{array}$ & $\begin{array}{c}\text { EDIJCATION } \\
2.220 \\
2: 229 \\
0.995 \\
1: 417 \\
0.774 \\
3: 197 \\
0.945 \\
1: 127 \\
0.825 \\
1.765\end{array}$ \\
\hline
\end{tabular}

\begin{tabular}{|c|c|c|c|c|c|}
\hline 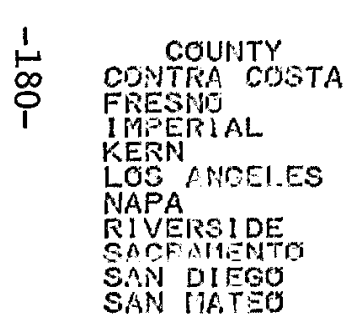 & $\begin{array}{l}\text { PUBL10 } \\
\text { WAYS } \\
1.573 \\
1.694 \\
1.953 \\
1.537 \\
0.851 \\
2.241 \\
1.260 \\
1.000 \\
0.991 \\
0.993\end{array}$ & $\begin{array}{c}\text { RECFEATI INN } \\
0.032 \\
0.507 \\
0.603 \\
1.915 \\
1.116 \\
0.209 \\
0.409 \\
0.998 \\
0.697 \\
0.910\end{array}$ & $\begin{array}{l}\text { DEET } \\
0.878 \\
0: \\
0.1 \\
1.167 \\
0.746 \\
3.838 \\
0.384 \\
2: 549 \\
2.617 \\
0.300\end{array}$ & $\begin{array}{c}\text { CONTINGENCY } \\
\text { Q1ESERVE } \\
1.513 \\
1.839 \\
1.627 \\
1.824 \\
0.619 \\
8.735 \\
0.975 \\
3.315 \\
0.7106 \\
1.228\end{array}$ & $\begin{array}{l}\text { GEN GOVT } \\
\text { PLANT ACQUI. } \\
0.413 \\
0.543 \\
0.551 \\
0.386 \\
1.170 \\
0.672 \\
0.856 \\
0.586 \\
1.133 \\
0.384\end{array}$ \\
\hline
\end{tabular}

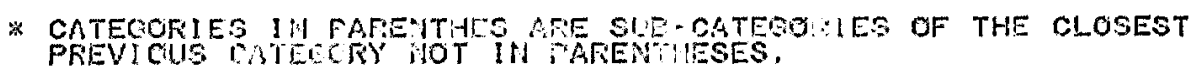


Table 7.3. Comparative percentage composition of selected county budgets for 1975-76.

\begin{tabular}{|c|c|c|c|c|c|c|c|c|}
\hline County & $\begin{array}{l}\text { General } \\
\text { government }\end{array}$ & $\left(\begin{array}{c}\text { Plant } \\
\text { acquisition }\end{array}\right)^{a}$ & $\begin{array}{l}\% \text { of Tota } \\
\text { Public } \\
\text { protection }\end{array}$ & (Courts) & (Police) & (Fire) & $\begin{array}{l}\text { Health and } \\
\text { sanitation }\end{array}$ & (Health) \\
\hline Contra Costa & 8.483 & 0.863 & 18.522 & 5.220 & 4.261 & 0 & 16.222 & 16.020 \\
\hline Fresno & 16.591 & 6.562 & 17.065 & 4.584 & 4.717 & 0 & 8.635 & 8.508 \\
\hline Imperial & 29.498 & 19.325 & 20.302 & 4.269 & 4.430 & 1.929 & 6.540 & 5.454 \\
\hline Kern & 12.685 & 5.552 & 25.067 & 3.614 & 5.569 & 8.424 & 16.022 & 15.484 \\
\hline Los Angeles & 22.610 & 1.002 & 14.002 & 4.019 & 3.952 & 0.855 & 16.212 & $15.839^{\circ}$ \\
\hline Napa & 19.086 & 6.670 & 19.980 & 3.695 & 5.319 & 2.168 & 8.554 & 8.436 \\
\hline RIverside & 18.836 & 3.029 & 21.935 & 4.460 & 6.361 & 1.335 & 8.595 & 7.785 \\
\hline Sacramento & 13.018 & 2.193 & 21.361 & 6.193 & 6.298 & 0 & 14.450 & 14.442 \\
\hline San Diego & 26.093 & 5.165 & 17.805 & 5.710 & 4.918 & 0 & 7.635 & 6.186 \\
\hline San Mateo & 14.870 & 7.775 & 22.724 & 5.438 & 1.218 & 0.481 & 19.947 & 19.872 \\
\hline County & (Sanitation) & $\begin{array}{c}\text { Public } \\
\text { assistance }\end{array}$ & Education & $\begin{array}{l}\text { Public } \\
\text { ways }\end{array}$ & Recreation & Debt & $\begin{array}{c}\text { Reserve } \\
\text { and } \\
\text { contingency }\end{array}$ & $\begin{array}{l}\text { General } \\
\text { government } \\
\text { less plant } \\
\text { acquisition }\end{array}$ \\
\hline Contra Costa & 0.202 & 44.498 & 2.362 & 7.783 & 0.045 & 0.210 & 1.875 & 7.620 \\
\hline Fresno & 0.126 & 43.946 & 2.372 & 8.383 & 0.720 & 0 & 2.279 & 10.029 \\
\hline Imperial & 1.085 & 30.065 & 1.059 & 9.665 & 0.856 & 0 & 2.016 & 10.173 \\
\hline Kern & 0.533 & 31.849 & 1.508 & 7.609 & 2.718 & 0.279 & 2.260 & 7.133 \\
\hline Los Angeles & 0.373 & 38.574 & 0.823 & 4.210 & 1.627 & 0.178 & 0.767 & 21.607 \\
\hline Napa & 0.058 & 25.819 & 3.370 & 11.093 & 0.296 & 0.916 & 10.886 & 12.413 \\
\hline Riverside & 0,810 & 41.426 & 1.006 & 6.236 & 0.666 & 0.092 & 1.208 & 15.807 \\
\hline Sacramento & 0.008 & 39.260 & 1.200 & 4.951 & 1.402 & 0.608 & 4.107 & 10.825 \\
\hline San Diego & 1.449 & 40.135 & 0.878 & 4.903 & 0.989 & 0.624 & 0.937 & 20.928 \\
\hline San Mateo & 0.075 & 32.931 & 1.879 & 4.914 & 1.150 & 0.072 & 1.521 & 7.096 \\
\hline
\end{tabular}


Table 7.4. County fiscal per capita budgets.

\begin{tabular}{|c|c|c|c|c|c|c|c|c|c|c|c|}
\hline 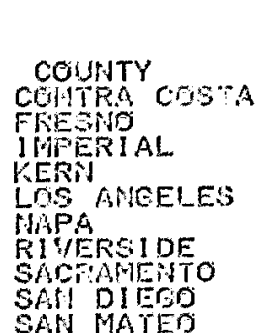 & $\begin{array}{r}\text { TOTAL } \\
\text { BUDEET } \\
372.05 \\
404.55 \\
519.82 \\
490.66 \\
404.96 \\
313.02 \\
350.91 \\
385.09 \\
314.36 \\
279.78\end{array}$ & $\begin{array}{r}\text { GEM. } \\
65 V T \\
31.56 \\
6 \%: 12 \\
153.33 \\
52.24 \\
91.56 \\
59.74 \\
69.10 \\
50: 13 \\
82.03 \\
41.50\end{array}$ & $\begin{array}{c}\text { (PLANT } \\
\text { A.0OU1) } \\
3.21 \\
26.55 \\
100.45 \\
27.24 \\
4.06 \\
20.08 \\
10.63 \\
8.45 \\
16.24 \\
21.75\end{array}$ & $\begin{array}{l}\text { PUBLIC } \\
\text { FRETECT } \\
69.91 \\
69.04 \\
105.53 \\
122.99 \\
56.70 \\
62.54 \\
76.97 \\
82.96 \\
55.97 \\
63.58\end{array}$ & $\begin{array}{l}\text { (COJETS) } \\
19.42 \\
13.54 \\
22.19 \\
17.73 \\
15.27 \\
11.57 \\
15.05 \\
23.85 \\
17.95 \\
15.21\end{array}$ & $\begin{array}{c}\text { (FCLICE) } \\
15.85 \\
19.08 \\
23.03 \\
27.32 \\
16.00 \\
16.65 \\
22.32 \\
24.25 \\
15.46 \\
3.41\end{array}$ & $\begin{array}{l}\text { (FIRE) } \\
0 .: \\
0: .03 \\
10.03 \\
41.33 \\
3.46 \\
6: 79 \\
4: 68 \\
0 . \\
0: \\
1.35\end{array}$ & $\begin{array}{c}\text { HEALTH } \\
\text { SA.H. } \\
60.36 \\
34.93 \\
34.00 \\
78.62 \\
65.65 \\
25.78 \\
30.16 \\
55.65 \\
24.00 \\
55.81\end{array}$ & $\begin{array}{l}\text { (HEALTH) } \\
59.60 \\
34.42 \\
28.35 \\
75.97 \\
64.14 \\
26.41 \\
27.32 \\
55.61 \\
19.45 \\
55.65\end{array}$ & $\begin{array}{l}(\text { SAN) } \\
0.75 \\
0.51 \\
5.64 \\
2.62 \\
1.51 \\
0.18 \\
2.84 \\
0.03 \\
4.56 \\
0.21\end{array}$ & $\begin{array}{r}\text { PUBL1C } \\
\text { ASS1ST } \\
165.56 \\
177.79 \\
156.28 \\
156.27 \\
156.21 \\
80.82 \\
145.37 \\
151.19 \\
126.17 \\
92.13\end{array}$ \\
\hline
\end{tabular}

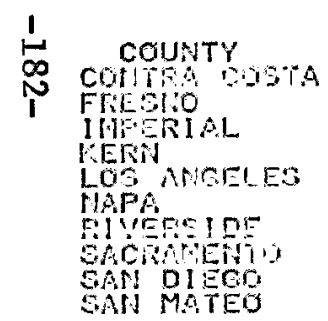

\begin{tabular}{|c|c|c|}
\hline $\begin{array}{c}\text { EOUCATIOH } \\
8.79 \\
9.60 \\
5.51 \\
7.40 \\
3.33 \\
10.55 \\
3.53 \\
4.62 \\
2.76 \\
5.26\end{array}$ & $\begin{array}{l}\text { PU. } \\
4.85 \\
20.96 \\
32.91 \\
50.24 \\
37.24 \\
17.05 \\
34.79 \\
31.89 \\
19.07 \\
15.41\end{array}$ & $\begin{array}{c}\text { RECREATIO. } \\
0.17 \\
2.91 \\
4.15 \\
13.34 \\
6.59 \\
0.93 \\
2.34 \\
5.40 \\
3.11 \\
3.22\end{array}$ \\
\hline
\end{tabular}

CONTIHEENGY GEN OOVT-

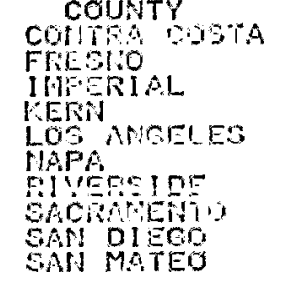

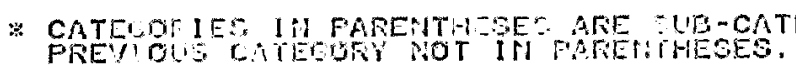

$\begin{array}{rr}6.98 & 23.35 \\ 9.22 & 40.57 \\ 10.48 & 52.88 \\ 11.09 & 35.00 \\ 3.10 & 87.50 \\ 31.07 & 38.86 \\ 4.24 & 55.47 \\ 15.92 & 49.68 \\ 2.95 & 65.79 \\ 4.20 & 19.85\end{array}$


Table 7.5. County per capita budget location quotients.

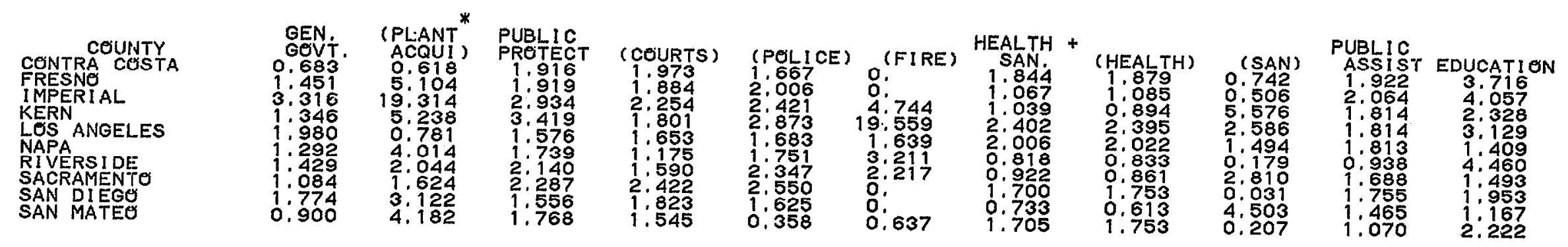

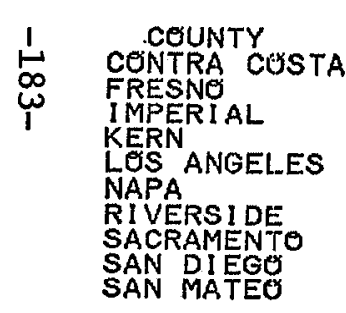

$\begin{array}{lc}\text { PUBLIC } & \\ \text { WAYS } & \text { RECREATION } \\ 2.632 & 0.053 \\ 3.083 & 0.923 \\ 4.567 & 1.410 \\ 3.394 & 4.228 \\ 1.550 & 2.089 \\ 3.156 & 0.294 \\ 1.989 & 0.741 \\ 1.733 & 1.712 \\ 1.401 & 0.986 \\ 1.250 & 1.020\end{array}$

$\begin{array}{lcc} & & \\ \text { DEBT } & \text { CONTINGENCY GEN, GOVT- } \\ \text { \& RESERVE } & \text { PLANT ACQU1, } \\ 1.469 & 2.533 & 0.691 \\ 0 . & 3.348 & 0.989 \\ 0 . & 3.805 & 1.288 \\ 2.577 & 4.026 & 0.853 \\ 1.358 & 1.127 & 2.132 \\ 5.405 & 12.372 & 0.947 \\ 0.607 & 1.539 & 1.352 \\ 4.416 & 5.743 & 1.016 \\ 3.701 & 1.070 & 1.603 \\ 0.378 & 1.545 & 0.484\end{array}$

* CATEGORIES IN PARENTHESES ARE SUB-CATEGORIES OF THE CLOSEST
PREVIOUS CATEGORY NOT IN PARENTHESES, 
Table 7.6. Imperial County revenues for FY 1975-76. ${ }^{1}$

\begin{tabular}{|c|c|c|c|c|}
\hline Category & Subcategory & $\begin{array}{c}\text { Subcaregory } \\
\text { total }\end{array}$ & Category total & Total revenus \\
\hline Current property tax & & & $\$ 7,164,567$ & 18.50 \\
\hline \multirow[t]{5}{*}{ other taxes } & & & $1,314,600$ & 3.39 \\
\hline & Other property tax & $\$ 225,100$ & & \\
\hline & Sales and use tax & 720,000 & & \\
\hline & In lieu livestock tax & $2 \mathrm{CO}, 000$ & & \\
\hline & Miscellaneous & 139,000 & & \\
\hline Licenses and permits & & & 104,180 & 0.27 \\
\hline $\begin{array}{l}\text { Fines, forfeitures, and } \\
\text { penialties }\end{array}$ & & & 638,095 & 1.65 \\
\hline Use of money and property & & & $1,031,120$ & 2.66 \\
\hline \multirow[t]{11}{*}{ State government } & & & $9,786,623$ & 25.27 \\
\hline & Highway users tax & $1,463,430$ & & \\
\hline & Gas tax & 326,736 & & \\
\hline & Transportation fund & 193,717 & & \\
\hline & In lieu taxes & 579,100 & & \\
\hline & Welfare & $1,934,500$ & & \\
\hline & Property tax deductions & 754,830 & & \\
\hline & Mental health & 630,000 & & \\
\hline & Aid for construction & $2,903,576$ & & \\
\hline & Parks and recreation & 237,035 & & \\
\hline & other & 713,699 & & \\
\hline \multirow[t]{6}{*}{ Federal government } & & & $9,578,533$ & 24.74 \\
\hline & Welfare & $1,025,000$ & & \\
\hline & Aid for children & $2,052,000$ & & \\
\hline & Ald for planning & $\$ 1,516,900$ & & \\
\hline & Geothermal project & 341,600 & & \\
\hline & other & $4,633,333$ & & \\
\hline Charges for current services & & & $\$ 2,133,856$ & 5.52 \\
\hline other & & & 99,180 & 0.26 \\
\hline Carry-over & & & $6,865,498$ & 17.73 \\
\hline Total & & & $\$ 38,722,551$ & 100.00 \\
\hline
\end{tabular}

remaining $27 \%, 11 \%$ came from charges for current services, $5 \%$ from use of money and property, $7 \%$ from other taxes, and the remaining $4+\%$ from miscellaneous sources.

\subsection{COUNTY TAX STRUCTURE}

There are four basic elements that comprise the total property tax rate for any given location: county, municipal, school, and special district taxes. The impact of special district taxes on any given location is highly variable within Imperial County, while the other three are fairly uniform. For any nonmunicipal area, the 1975-1976 county wide ta: rate is 2.9285 (per $\$ 100$ of assessed value). ${ }^{3}$ County wide tax rates within municipalities vary from a low of 2.7649 up to 2.9285 , while municipal taxes vary from a low of 


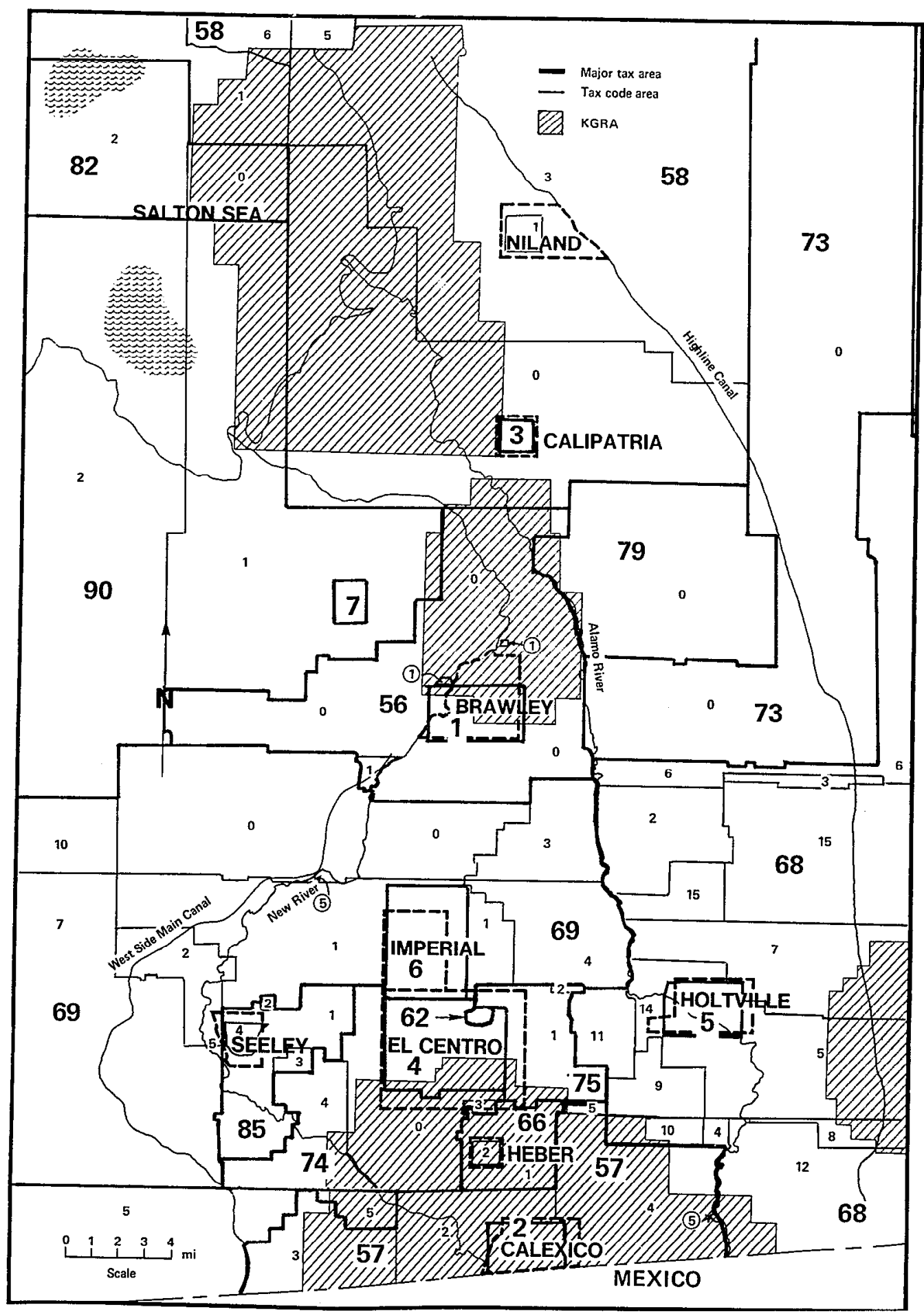

Fig. 7.1. Imperial County tax code areas mapped against KGRAs.

2.3200 in Calexico to a high of 4.1166 in Calipatria. School tax rates vary from 5.3625 to 7.3251 and, finally, cumulative special district taxes for the county tax code areas vary from 0 up to 11.9444 . Figure 7.1 shows the location of $\operatorname{tax}$ code areas with respect to the major 
KGRAs. This relationship is important since school, special districts, and, to a lesser extent, county tax revenues (and thus tax rates) will be significantly affected by geothermal development within their boundaries. Table 7.7 compares tax rates for the past 3 years for all tax code areas lying over, or adjacent to, major KGRAs. Previously anticipated reductions in the FY 77 county wide tax rate now appear unlikely, ${ }^{6}$ and the tax rates reported here for FY 75-76 will remain essentially unchanged for at least the immediate future. Currently, only $21.9 \%$ of the county's annual revenue is derived from in-county taxes (see Table 7.6), however this represents $44 \%$ of the FY 75-76 revenue that can be manipulated by the county. In addition, taxes are the easiest revenue item for the county to manipulate. Thus, while county tax rates are projected as near constant for the next 1 to 2 years, more generally they are subject to substantial change since the county in an attempt to control total county revenue tries to adjust to changing state and federal funding levels.

Analysis of the data in Table 7.7 reveals several interesting trends in Imperial County. With the exception of the general tax area 57-xxx, every tax code area shown in Table 7.7 either sustained a net tax rate decrease over the period shown or at least had a rate reduction for one of the years shom. This indicates a willingness on the part of local officials to translate increased assessed value and increased state and federal income into lower ta: rates rather than into increased local government spending. Over the 3-yr period shown, the largest total rate increase has been in ta: code area 66-002 (Heber) with a $30.5 \%$ increase. The second largest was 57-002 (Calexico) with an $18.8 \%$ increase. Of the 37 tax codc areas 1isted, 17 actually showed net reduced rates over this 3-yr poriod. Ceothermal development could affect these tax rates on several levels: First, county and state taxes associated with geothermal developmental activity could be transiated into either increased county spending for various servicus or into lower tax rates throughout the county. Either of these actions would affect all county citizens equally. Second, local and speclal districts co-located with geothermal development activity will accrue revenue from this development and, like the county, could either incrcalls the level of services provided (increasing spending) or reduce local tax rates. School tax rates, under SB-90, could decline with rovenues from geothermal development. This 

Table 7.7. Tax rate for tax code areas adjacent to major KGRAs
(1973-1976).3,4,5

\begin{tabular}{|c|c|c|c|c|c|}
\hline \multirow[b]{2}{*}{ Tax code } & \multirow[b]{2}{*}{ Area } & \multirow[b]{2}{*}{ KGRA } & \multicolumn{3}{|c|}{ Total tax rates } \\
\hline & & & $1973-4$ & $1974-5$ & $1975-6$ \\
\hline $01-001$ & Brawley & Brawley & 11.8437 & 12.5118 & 12.3549 \\
\hline $01-002$ & Brawley & Brawley & 11.8437 & 12.5118 & 12.3549 \\
\hline $02-000$ & Calexico & Heber & 10.6753 & 11.4181 & 11.4918 \\
\hline $02-001$ & Calexico & Heber & 10.6753 & 11.4181 & 11.4918 \\
\hline $03-000$ & Calipatria & Salton Sea & 14.9053 & 13.3301 & 13.5049 \\
\hline $04-000$ & El Centro & Heber & 12.5290 & 12.3289 & 12.0799 \\
\hline $04-001$ & E1 Centro & Heber & 12.5290 & 12.3289 & 12.0799 \\
\hline $04-002$ & E1 Centro & Heber & - & 12.3289 & 12.0799 \\
\hline $56-000$ & Brawley & Brawley & 8.4205 & 9.1070 & 8.9125 \\
\hline $57-001$ & Calexico & Heber & 7.8246 & 9.2622 & 9.0933 \\
\hline $57-002$ & Calexico & Heber & 7.8246 & 9.2622 & 9.2933 \\
\hline $57-003$ & Calexico & Heber & 7.8246 & 9.2622 & 9.2933 \\
\hline $57-004$ & Calexico & Heber & 7.8246 & 9.2622 & 9.2933 \\
\hline $57-005$ & Calexico & Heber & 7.9014 & 9.3659 & 9.2484 \\
\hline $58-000$ & Calipatria & Salton Sea & 9.9787 & 9.2135 & 9.3883 \\
\hline $58-001$ & Niland & Salton Sea & 9.7215 & 9.0684 & 9.3155 \\
\hline $58-003$ & Calipatria-Niland & Salton Sea & 9.6267 & 8.9536 & 9.1889 \\
\hline $58-005$ & Bombay Beach & Salton Sea & 11.2085 & - & 10.8299 \\
\hline $58-006$ & Bombay Beach & Salton Sea & 11.2085 & 10.2014 & 10.9565 \\
\hline $66-001$ & Heber & Heber & 9.2960 & 9.7837 & 9.4148 \\
\hline $66-002$ & Heber & Heber & 10.0239 & 13.5154 & 13.0811 \\
\hline $68-005$ & Holtville & East Mesa & 9.7655 & 8.5033 & 9.6839 \\
\hline $68-007$ & Pine Union & East Mesa & 9.6056 & 8.3663 & 9.5341 \\
\hline $68-008$ & Holtville & East Mesa & 9.6887 & 8.3996 & 9.5288 \\
\hline $68-009$ & Alamitos & Heber & 9.5573 & 8.3214 & 9.5341 \\
\hline $68-010$ & Alamitos & Heber & 9.4805 & 8.2177 & 9.5790 \\
\hline $68-012$ & Verde & $\begin{array}{l}\text { East Mesa/ } \\
\text { Heber }\end{array}$ & 9.4805 & 8.2177 & 9.5790 \\
\hline $68-013$ & Verde & Heber & 9.4805 & 8.2177 & 9.5790 \\
\hline $74-000$ & McCabe & Heber & 9.1324 & 8.9690 & 9.3009 \\
\hline $74-003$ & McCabe-Heber & Heber & 9.0497 & 8.8940 & 9.2427 \\
\hline
\end{tabular}




\begin{tabular}{|c|c|c|c|c|c|}
\hline \multirow[b]{2}{*}{ Tax code } & \multirow[b]{2}{*}{ Area } & \multirow[b]{2}{*}{ KGRA } & \multicolumn{3}{|c|}{ Total tax rate } \\
\hline & & & $1973-4$ & $1974-5$ & $1975-6$ \\
\hline $74-004$ & McCabe & Heber & 9.0497 & 8.8940 & 9.2427 \\
\hline $74-005$ & McCabe-Centinel & Heber & 8.9729 & 8.7903 & 9.2876 \\
\hline $75-001$ & Meadows & Heber & 10.1443 & 9.7445 & 10.2521 \\
\hline $79-000$ & Mulberry & Brawley & 9.5324 & 9.0425 & 9.2122 \\
\hline $82-000$ & Oasis & Salton Sea & 10.2188 & 9.7055 & 9.7809 \\
\hline $82-002$ & Oasis & Salton Sea & 10.1240 & 9.5907 & 9.6543 \\
\hline $90-001$ & Westmorland & Brawley & 9.0925 & 9.2033 & 9.0149 \\
\hline
\end{tabular}

KGRA

Salton Sea

Brawley

Heber

East Mesa represents a local benefit that would accrue only to those in the areas surrounding the development site. Finally, geothermal development will bring new jobs and population into the county. This new population will represent a demand for increased local and county governmental services. If these new people do not choose to live in the tax code areas immediately surrounding associated development sites, their demand for services will not be realized by the same local, school, and special districts that will accrue geothermal tax benefits.
For example, if vorkers from a geothermal power plant in the Brawley KGRA decided to live in E1 Centro, the El Centro school districts would feel the increased demand for services while Brawley Elementary and Brawley Union High School Districts would receive the additional ta: monies From the development. Finally, the number of tax code areas potentially affected by development on each FigRA is listed in the Summary section of Table 7.7. Development in the Heber KGRA rill have the most diverse impact while development in other major KGRAs 
will have a much more spatially limited impact. Only one tax-code area, 68-012 is positioned to receive tax benefits from more than one KGRA.

\subsection{COUNTY SCHOOL DISTRICTS AND BUDGETS}

There are 17 school districts in Imperial county. Of these, nine are elementary school districts with two superimposed high school districts, five are unified school districts, and the last is the Imperial Community College district, which is county wide. The boundaries of these various districts are shown in Fig. 7.2. for the Imperial Valley and in Fig. 7.3 are mapped against KGRA boundaries. This relationship will be extremely important to property owners for state school reveune limits will increase the probability that geothermal tax revenues to school districts will be translated into lower tax rates.

Selected fiscal data from school districts in Imperial County are presented in Table 7.8. Annual expenditures per average daily attendance (ADA) were around $\$ 1000$ for elementary schools with the exception of Magnolia Union (\$2067.46) and Mulberry (\$1578.57). However, the elementary school tax rate for both of these districts was low, with Magnolia Union having the lowest tax rate (1.9656) of any of the 16 basic school districts. The cause of this apparent anomaly has not yet been determined. Annual expenditure per ADA was only marginally higher for high school districts (\$1256) than for elementary schools (\$1212). Unified districts, however, spend considerably more per year per $\mathrm{ADA}$ with an average of $\$ 1343$ per ADA per year than either of the other two types of school districts. The basis for the higher level of unified district spending has not yet been determined. Similarly, unified districts averaged slightly higher tax rates (4.9821) than areas with separate elementary and high school districts for which average rates were 2.9321 and 2.0003, respectively, for a total of 4.9324. The revenue limit for each district, based on federal revenue limit criteria, is listed in column 5, Table 7.8. This limit will be a primary determinant of the impact on tax rates of geothermal development.

Trends in the cost of education per ADA are shown by school district in Table 7.9. Ten-year trends showing the slowest rate of cost increase have been in high school education for which the cost has doubled. Elementary school education in general tripled over this 10-yr period with Magnolia Union showing the largest increase at $364.7 \%$. Over $250 \%$ of this increase however was recorded in FY 1974-1975; cost increases for 


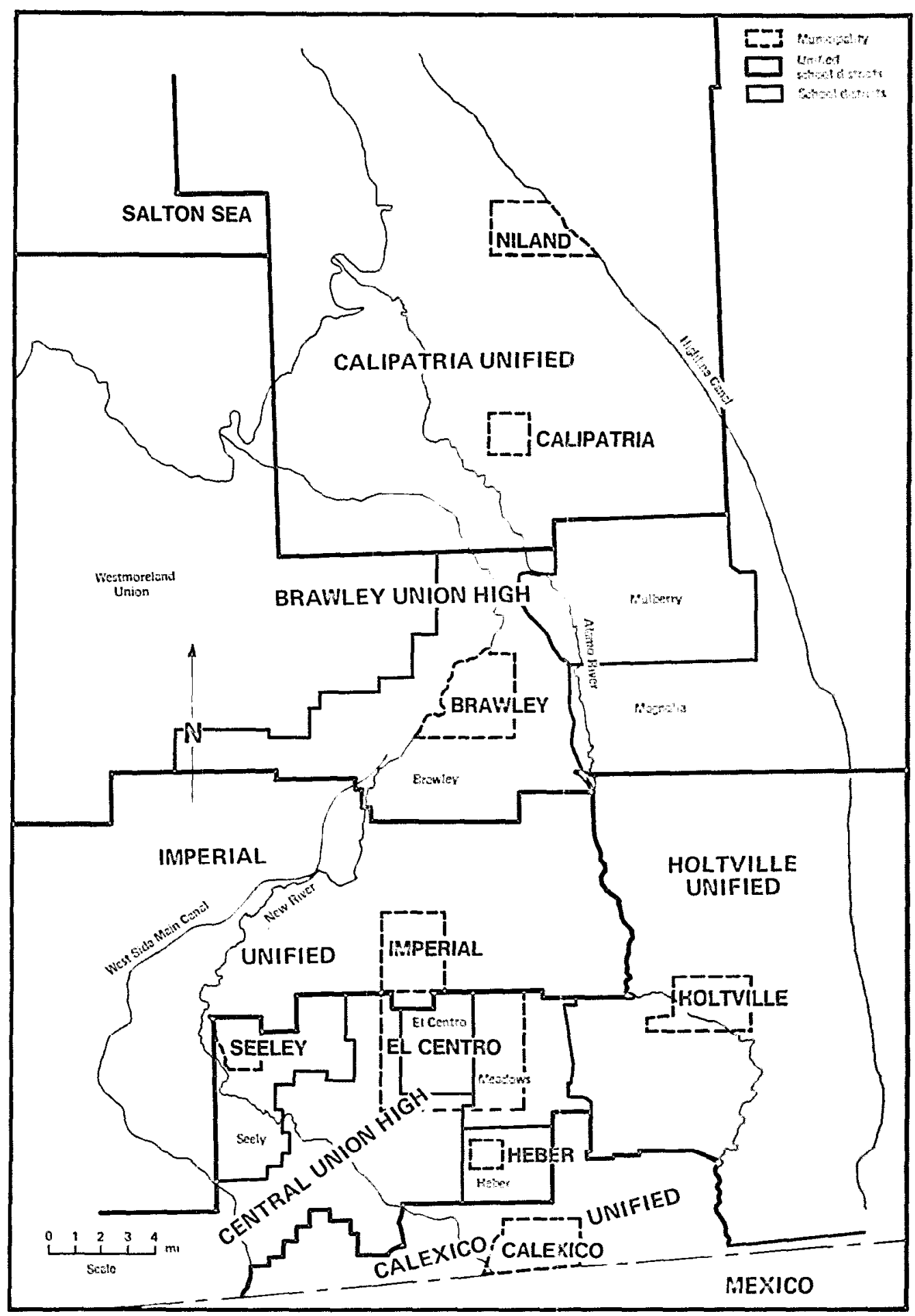

Fig. 7.2. Imperial county school districts. ${ }^{7}$ 


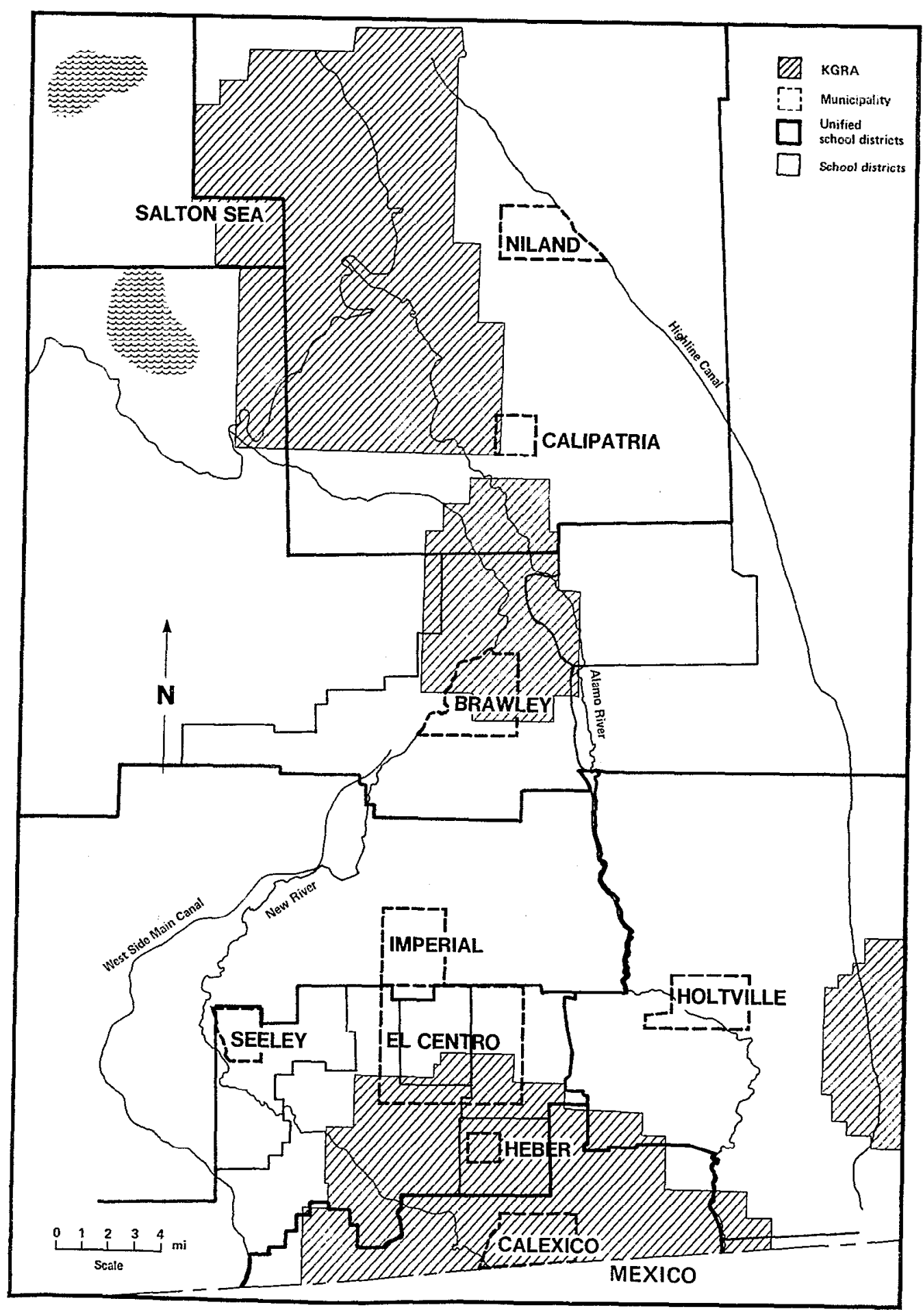

Fig. 7.3. Imperial County school districts mapped against KGRAs. 7 
Table 7.8. Imperial County school district fiscal summary for FY 1974-75. 7

\begin{tabular}{|c|c|c|c|c|c|c|}
\hline $\begin{array}{l}\text { School } \\
\text { district }\end{array}$ & $\begin{array}{c}\text { Total } \\
\text { district } \\
\text { school tax } \\
\text { rate }\end{array}$ & $\begin{array}{c}\text { District } \\
\text { assessed } \\
\text { value } \\
(x \$ 1000)\end{array}$ & $\begin{array}{c}\text { Value } \\
\text { secured } \\
\text { for tax rate } \\
(x \$ 1000)\end{array}$ & $\begin{array}{c}\text { Annual } \\
\text { ADA }\end{array}$ & $\begin{array}{c}\text { Total } \\
\text { revenue } \\
\text { limit (using } \\
\text { actual data) }\end{array}$ & $\begin{array}{c}\text { Total } \\
\text { funds } \\
\text { available }\end{array}$ \\
\hline Brawley $(E)^{a}$ & 2.3102 & 34,774 & 30,991 & 3,553 & $2,892,010$ & $4,161,287$ \\
\hline E1 Centro (E) & 2.7370 & 45,728 & 41,373 & 4,270 & $3,576,967$ & $4,755,848$ \\
\hline Heber $(E)$ & 3.6987 & 3,848 & 3,103 & 708 & 596,507 & 957,235 \\
\hline Magnolia (E) & 1.9656 & 5,734 & 5,613 & 67 & 56,880 & 211,640 \\
\hline McCabe (E) & 2.8644 & 11,824 & 10,173 & 285 & 204,001 & 381,430 \\
\hline Meadows (E) & 3.8156 & 11,137 & 10,591 & 269 & 311,644 & 479,242 \\
\hline Mulberry (E) & 2.6113 & 6,967 & 6,559 & 73 & 60,701 & 177,942 \\
\hline Seeley (E) & 3.9722 & 4,401 & 3,635 & 496 & 421,549 & 896,826 \\
\hline Vestmorland (E) & 2.4140 & 11,389 & 10,455 & 443 & 332,540 & 484,975 \\
\hline Brawley (H) & 2.0057 & 58,864 & 53,616 & 1,583 & $1,690,729$ & $2,277,079$ \\
\hline Central (H) & 1.9948 & 76,938 & 68,804 & 2,376 & $2,336,917$ & $2,982,178$ \\
\hline Calexico (U) & 4.8006 & 28,691 & 24,837 & 4,703 & $4,258,817$ & $6,027,130$ \\
\hline Calipatria (U) & 4.9197 & 20,159 & 19,482 & 1,217 & $1,182,640$ & $1,965,098$ \\
\hline Holtville (U) & 5.2405 & 34,019 & 31,562 & 1,956 & $1,871,307$ & $2,997,740$ \\
\hline Imperial (U) & 4.0040 & 34,410 & 30,568 & 1,569 & $1,484,218$ & $2,474,635$ \\
\hline San Pasqual (U) & 5.9456 & 7,227 & 6,691 & 638 & 711,520 & $1,465,526$ \\
\hline ICC (C) & 0.8810 & 260,299 & 235,513 & 2,808 & $3,325,425$ & $8,011,109$ \\
\hline
\end{tabular}

$a_{E}=$ elezentary school district; $H=$ high school district; $U$ = unified school district; $c=$ com 
Table 7.9. Current cost ${ }^{a}$ of education per A.D.A. in Imperial County (1964-1974). 7

\begin{tabular}{|c|c|c|c|c|c|c|}
\hline School districts: & $1964-65$ & $1965-66$ & $1966-67$ & $1967-68$ & $1968-69$ & $1969-7 C$ \\
\hline \multicolumn{7}{|l|}{ Elementary: } \\
\hline Brawley & 358.23 & 384.28 & 380.67 & 409.42 & 477.93 & 538.47 \\
\hline E1 Centro & 375.96 & 456.84 & 473.75 & 498.10 & 548.63 & 577.35 \\
\hline Heber & 363.59 & 337.03 & 391.63 & 429.89 & 508.86 & 560.19 \\
\hline Magnolia Union & 444.92 & 534.08 & 549.96 & 530.18 & 555.66 & 608.77 \\
\hline McCabe Union & 414.26 & 468.50 & 448.69 & 488.62 & 531.54 & 567.00 \\
\hline Meadows Union & 395.37 & 414.34 & 457.09 & 502.21 & 618.80 & 733.40 \\
\hline Mulberry & 535.58 & 654.66 & 628.52 & 572.62 & 810.16 & 914.24 \\
\hline Seeley Union & 278.45 & 350.31 & 432.56 & 488.21 & 495.71 & 581.11 \\
\hline Westmorland Union & 316.85 & 422.65 & 435.82 & 460.14 & 536.29 & 569.37 \\
\hline Elementary averages & 368.08 & 458.87 & 462.52 & 492.14 & 522.06 & 627.77 \\
\hline \multicolumn{7}{|l|}{ High school: } \\
\hline Brawley Union & 660.63 & 696.18 & 801.49 & 798.05 & 835.73 & 928.75 \\
\hline Central Union & 586.57 & 640.12 & 695.72 & 707.97 & 747.00 & 786.57 \\
\hline High school averages & 599.12 & 672.24 & 748.60 & 753.01 & 784.26 & 857.66 \\
\hline \multicolumn{7}{|l|}{ Unified: } \\
\hline Calexico & 461.78 & 522.92 & 470.28 & 529.20 & 628.99 & 655.92 \\
\hline Calipatria & 541.24 & 518.35 & 591.36 & 547.36 & 641.04 & 698.80 \\
\hline Ho1tville & 735.36 & 829.76 & 608.49 & 644.13 & 700.96 & 742.48 \\
\hline Imperial & 422.26 & 461.45 & 525.35 & 550.32 & 611.11 & 713.27 \\
\hline San Pasqual Valley & 441.65 & 581.49 & 626.04 & 711.14 & 670.36 & 696.85 \\
\hline Unified averages & 463.88 & 520.43 & 564.30 & 596.43 & 644.96 & 701.46 \\
\hline Imperial Community colleges & 662.21 & 659.22 & 686.99 & 790.51 & 820.68 & 904.01 \\
\hline
\end{tabular}

$\overline{\text { Excludes cafeterias, community services and capital outlays. }}$ 
Table 7.9. (Concluded)

\begin{tabular}{|c|c|c|c|c|c|c|}
\hline School districts: & $1970-71$ & $1971-72$ & $1972-73$ & $1973-74$ & $1974-75$ & \\
\hline \multicolumn{7}{|l|}{ Elementary: } \\
\hline Erawley & 577.23 & 625.92 & 538.88 & 795.55 & 953.91 & + \\
\hline El Centro & 636.37 & 668.10 & 730.84 & 843.32 & $1,028.68$ & + \\
\hline Heber & 575.29 & 642.05 & 619.48 & 911.99 & $1,076.23$ & + \\
\hline Magno1ia Union & 869.78 & 720.47 & 739.48 & 882.69 & $2,067.46$ & + \\
\hline NeCabe Union & 662.19 & 718.61 & 756.30 & 937.13 & $1,089.37$ & + \\
\hline Meadows Union & 907.89 & 912.24 & 993.26 & $1,093.65$ & $1,211.22$ & + \\
\hline Yulberry & 826.53 & $1,032.24$ & $1,343.14$ & $1,098.80$ & $1,578.57$ & + \\
\hline Seeley Union & 611.64 & 645.17 & 711.69 & 838.57 & 933.16 & + \\
\hline Vestmorland Union & 584.32 & 709.50 & 654.79 & 859.16 & 969.68 & + \\
\hline E1cmentary averages & 620.59 & 662.32 & 700.32 & 843.13 & $1,015.91$ & + \\
\hline \multicolumn{7}{|l|}{ High school: } \\
\hline Branley Unien & 923.94 & 930.24 & $1,055.68$ & $1,139,58$ & $1,326.59$ & + \\
\hline Centra1 Union & 795.28 & 887.39 & 975.79 & $1,062.27$ & $1,185.67$ & + \\
\hline High school averages & 848.02 & 912.67 & $1,008.40$ & $1,093.95$ & $1,241.86$ & + \\
\hline \multicolumn{7}{|l|}{ Unified: } \\
\hline Calcico & 691.59 & 742.82 & 762.50 & 870.32 & $1,121.40$ & + \\
\hline Calipatria & 701.97 & 806,82 & 855.64 & $1,023.16$ & $1,219.33$ & 4 \\
\hline Holtville & 770.13 & 806.97 & 905.19 & $1,030.7 ?$ & $1,176.86$ & $\therefore$ \\
\hline Imperial & 698.52 & 786.60 & 875.45 & $1,050.21$ & $1,425.03$ & $\rightarrow$ \\
\hline Sin Pasqual valley & 803.80 & 968.14 & $1,071.34$ & $1,359.83$ & $1,770.08$ & 4 \\
\hline Unified ayerages & 717.02 & 786.36 & 840.45 & 978.52 & $1,234.01$ & 4 \\
\hline Itperiul cumunity colleges & 979.49 & $1,093.65$ & $1,199,69$ & $1,163.66$ & $1,926.85$ & 4 \\
\hline
\end{tabular}


Magnolia Union before that year were slightly below the average elementary school increase. Unified school districts showed the least consistency in recorded cost increases, varying from a low of $+60 \%$ (Holtville) to a high of $+300 \%$ (San Pasqual Valley). The average for unified school districts $(+166.0 \%)$ was slightly below the elementary school average $(+176.0 \%)$.

Table 7.10 shows assessed value per total ADA for each school district and the percentage change of this value over the past 5-yr period. Two significant facts are presented on this table. First, there is wide variation in the assessed value per ADA among school districts; this variation appears to be increasing rather than decreasing. Second, the assessed value of four districts has actually declined over the past five years, and in only one of these four cases, Heber Elementary, has there been a recent upward trend toward value recovery. The positive impact of geothermal development can potentially be felt most strongly in districts with decreasing assessed value or in districts with low assessed value per ADA.

As seen in Figs. 7.2 and 7.3, not all school districts stand an equal chance for receiving tax benefit from geothermal development. Imperial Unified, San Pasqual Valley Unified, and Seeley Elementary
School Districts do not overlap with any of the KGRAs. Magnolia Elementary, Mulberry Elementary, Westmorland Elementary, and E1 Centro Elementary School Districts have extremely limited overlap with KGRAs. Almost al1 of the overlap of the Mulberry District with the Brawley KGRA 1ies within a zone designated by the county for preservation. E1 Centro Elementary School District's overlap with the Heber KGRA is all within municipal boundaries, thus can not be considered for geothermal plant siting. The remaining nine school districts will be the principal beneficiaries of geothermal development. Calipatria Unified School District covers essentially all of the Salton Sea KGRA and the northern third of the Brawley KGRA. Brawley Union High School and Brawley Elementary School jointly cover the southern two-thirds of the Brawley KGRA. Five districts (Calexico Unified, Central Union High, McCabe Elementary, Heber Elementary, and Meadows Elementary) split coverage of the Heber KGRA with Calexico Unified District having the largest land overlap. Holtville Unified District covers all of the East Mesa KGRA, has several small overlaps with the Heber KGRA, and also completely contains two smaller, lower potential KGRAs, the Dunes and Glamis KGRAs (both located east of Imperial Valley). 
Table 7.10. Imperial County school district assessed value ${ }^{a}$ (1970-1975). 7

\begin{tabular}{|c|c|c|c|c|c|c|}
\hline School districts: & $1970-71$ & $1971-72$ & $1972-73$ & $1973-74$ & $1974-75$ & $\begin{array}{l}y x^{5} \% \\
\text { change }\end{array}$ \\
\hline \multicolumn{7}{|l|}{ Elementary: } \\
\hline Brawley & 7,178 & 7,295 & 7,829 & 8,143 & 9,320 & +29.8 \\
\hline E1 Centro & 7,241 & 7,472 & 3,495 & 8,645 & 9,002 & +24.3 \\
\hline Heber & 7,057 & 5,938 & 4,834 & 4,532 & 5,256 & -25.5 \\
\hline Magnolia Union & 47,848 & 50,910 & 47,948 & 52,538 & 75,028 & +56.8 \\
\hline McCabe Union & 31,416 & 35,091 & 32,603 & 32,829 & 37,882 & +20.6 \\
\hline Meadows Union & 26,795 & 28,113 & 37,541 & 36,591 & 34,042 & +27.0 \\
\hline Mulberry & 63,656 & 70,185 & $7 \div, 699$ & 54,588 & 79,462 & +24.8 \\
\hline Seeley Union & 5,764 & 6,268 & 7,483 & 7,700 & 8,215 & +42.5 \\
\hline Westmorland Union & 20,908 & 21,825 & 20,704 & 21,320 & 21,070 & +0.8 \\
\hline \multicolumn{7}{|l|}{ High school: } \\
\hline Brawley Union & 29,597 & 29,126 & 30,886 & 30,925 & 34,271 & +15.8 \\
\hline Central Union & 23,352 & 23,776 & 25,927 & 27,138 & 28,143 & +20.5 \\
\hline \multicolumn{7}{|l|}{ Unified: } \\
\hline $\begin{aligned} & \text { Calexico-ron } \text { Elementary } \\
& \text { High school }\end{aligned}$ & $\begin{array}{r}6,679 \\
14,486\end{array}$ & $\begin{array}{r}7,203 \\
15,758\end{array}$ & $\begin{array}{r}7,177 \\
16,312\end{array}$ & $\begin{array}{r}6,777 \\
15,932\end{array}$ & $\begin{array}{r}8,906 \\
20,484\end{array}$ & $\begin{array}{l}+33.3 \\
+41.4\end{array}$ \\
\hline $\begin{array}{r}\text { Calipatria-m- } \\
\text { High school }\end{array}$ & $\begin{array}{l}19,803 \\
56,976\end{array}$ & $\begin{array}{l}20,247 \\
55,696\end{array}$ & $\begin{array}{l}21,800 \\
54,855\end{array}$ & $\begin{array}{l}22,326 \\
51,208\end{array}$ & $\begin{array}{l}23,754 \\
49,817\end{array}$ & $\begin{array}{l}+20.0 \\
+12.6\end{array}$ \\
\hline $\begin{array}{r}\text { Holtville-ron } \\
\text { High school }\end{array}$ & $\begin{array}{l}20,401 \\
42,866\end{array}$ & $\begin{array}{l}20,369 \\
40,494\end{array}$ & $\begin{array}{l}21,008 \\
44,407\end{array}$ & $\begin{array}{l}21,447 \\
43,433\end{array}$ & $\begin{array}{l}22,194 \\
47,806\end{array}$ & $\begin{array}{l}+8.8 \\
+11.5\end{array}$ \\
\hline $\begin{array}{r}\text { Imperial-_- } \\
\text { High school }\end{array}$ & $\begin{array}{l}21,109 \\
57,495\end{array}$ & $\begin{array}{l}20,959 \\
58,033\end{array}$ & $\begin{array}{l}22,529 \\
60,367\end{array}$ & $\begin{array}{l}23,801 \\
58,634\end{array}$ & $\begin{array}{l}26,928 \\
61,623\end{array}$ & $\begin{array}{r}+27.6 \\
+7.2\end{array}$ \\
\hline $\begin{array}{r}\text { San Pasqual Valley-Elementary } \\
\text { High school }\end{array}$ & $\begin{array}{l}14,373 \\
53,162\end{array}$ & $\begin{array}{l}14,333 \\
49,732\end{array}$ & $\begin{array}{l}15,733 \\
54,216\end{array}$ & $\begin{array}{l}16,409 \\
51,108\end{array}$ & $\begin{array}{l}17,236 \\
50,530\end{array}$ & $\begin{array}{l}+19.9 \\
-5.0\end{array}$ \\
\hline Imperial Community College & 100,821 & 102,106 & 111,631 & 91,811 & 83,259 & -17.4 \\
\hline
\end{tabular}

$\mathrm{a}_{\text {Numbers }}$ listed are assessed valuation per total ADA.

\subsection{IMPERIAL COUNTY LAND USE PLANNING}

Existing county land use plans and the locations of state and federal wildlife areas could significantly restrict the spatial development of geothermal energy. The principal effect of these restrictions will be to concentrate future large-scale geothermal activity. It has not been determined whether these spatial restrictions will significantly impede ultimate resource development. There are three sources of land-use restrictions that are addressed by our study program: county zoning ordinances; the county Ultimate Land Use Plan; and proposed county regulation governing geothermal operations, plant permitting, and 
plant siting within Imperial County (similar to that for well drilling operations ${ }^{8}$ ). Once the Geothermal Element for the County General Plan has been adopted, it will replace the last of these three regulations and will clarify the relationship between geothermal erergy and the first two regulatory elements.

Zoning Ordinances

Imperial County zoning codes now provide an overlay zone designation $G$ to indicate that geothermal activity is allowable within that general zone. To date this designation has been applied only to major test and demonstration plant sites in the Salton Sea KGRA. Adoption of a geothermal element to the county general plan will clarify the application of this overlay zone designation. In the absence of this elemen' a preliminary review of the county zone codes indicates that geothermal activity would now be excluded from the following zones: a11 residential zones $\mathrm{R}-1, \mathrm{R}-1-\mathrm{T}$, $\mathrm{R}-2, \mathrm{R}-3, \mathrm{R}-4$, and $\mathrm{R}-4-\mathrm{T}$; agricultural zones $\mathrm{R}-\mathrm{A}$ and $\mathrm{A}-1$; a11 commercial zones $\mathrm{C}-0, \mathrm{C}-1$, and $\mathrm{C}-2$; manufacturing zone $\mathrm{M}-1$; recreation zones $\mathrm{F}$; and the open space zones $S$. This leaves the following zones available for location of geothermal development: agricultural zones $\mathrm{A}-2, \mathrm{~A}-3$, and $\mathrm{A}-2-\mathrm{R}$ and manufacturing zone $\mathrm{M}-2$.
Those county zones that would restrict geothermal development are mapped against county boundaries in Fig. 7.4. Existing zonj..1g patterns will create only minor rescrictions on overall development. The restricted areas comprise primarily city boundaries and recreational zones with the largest recreational zone being along the Salton Sea shore line.

U1timate Land Use Plan

Assuming that Imperial County will retain its existing Ultimate Land Use $\mathrm{Plan}$ and require compatability of geothermal development with this plan, we mapped ultimate land use against KGRA boundaries in Fig. 7.5. Land use zones that would exclude geothermal development are urban, rural residential, recreation, preservation, and special public areas. Since no industrial or desert residential areas overlay major KGRAs, this plan places al1 geothermal development on general agricultural lands.

\section{Geothermal Element}

County regulations for permitting and siting of geothermal powerplants are still being formulated. Restrictions for such facilities generally require minimum distances between the plant and community boundaries, schools, hospitals, feed lots, etc. Figure 6.4 shows the relationship 


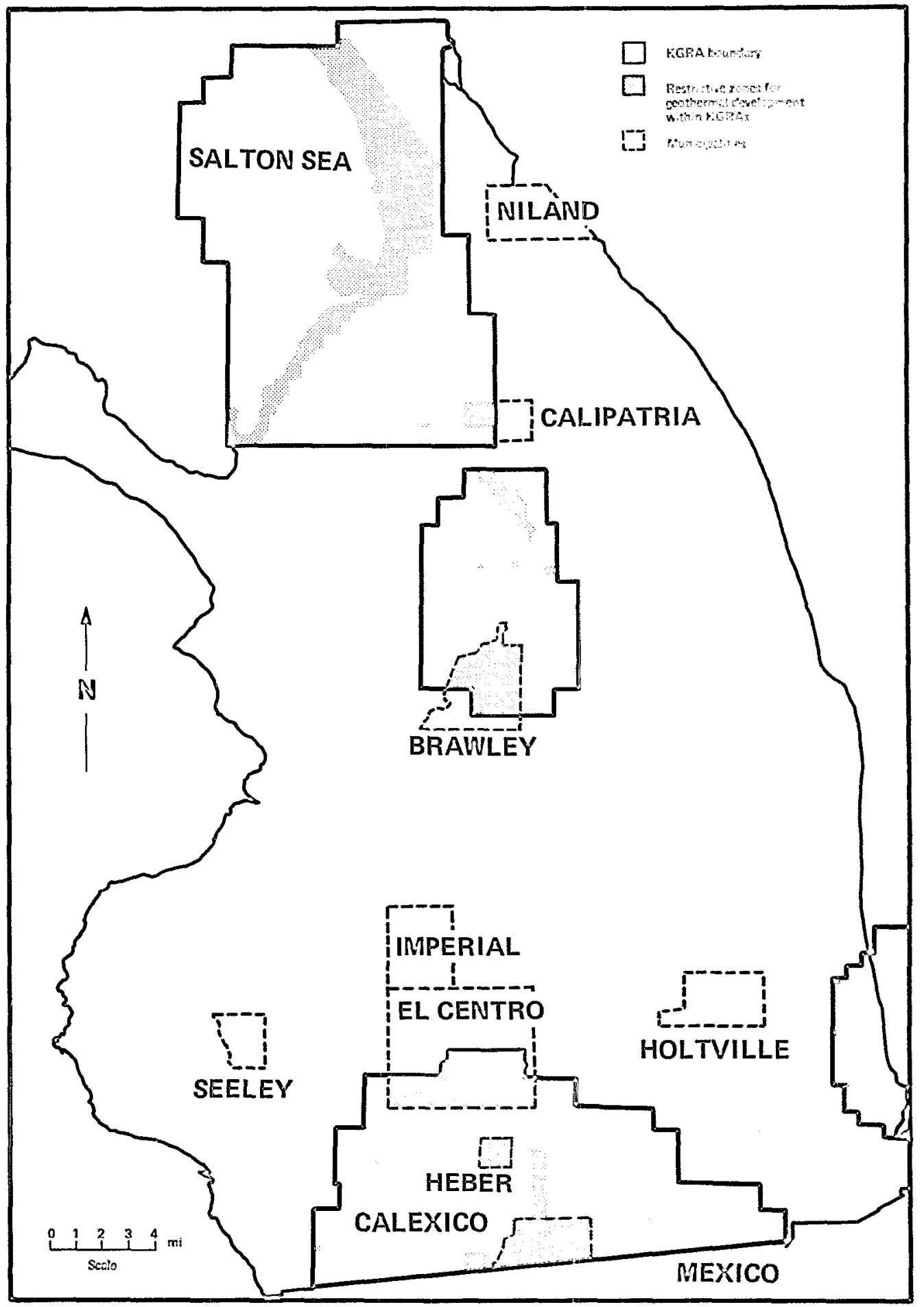

Fig. 7.4. Imperial County restrictive land use zones mapped against likA boundaries. 9 


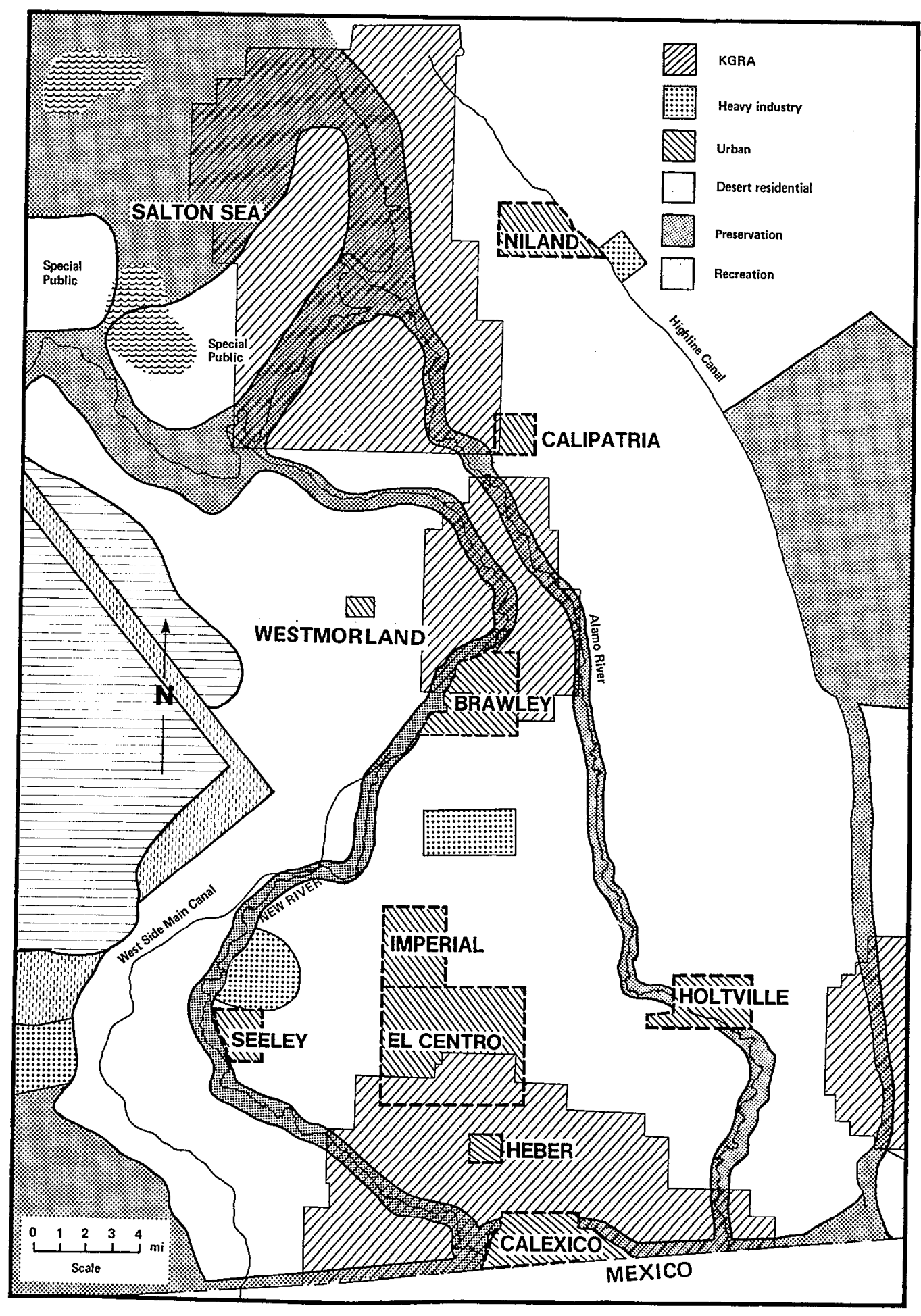

Fig. 7.5. Imperial County ultimate land use plan mapped against KGRAs. 10 
between KGRAs and a11 feed lots within the county. Application of minimum distance requirements to the feed lot distributions would create several exclusion zones of significant size for geothermal development.

The net effect of these land use restrictions would force geothermal development to compete almost exclusively with agriculture for land use. The significance and magnitude of this competition will be a function of the number of plants to be located in each KGRA, of spacings of wells and reinjection wells, and requirements for and patterns of brine piping. These factors are, in turn, a function of the extent to which geothermal development takes place.

\subsection{INTRACOUNTY FISCAL DESCRIPTION Municipal Services}

Individual city budgets were not analyzed for this report. However, most Imperial County cities are relatively sma11 (population of 10,000 or less); for units of that size, per capita fiscal budget analysis is less reliable for predicting future fiscal expenditures than it is for large units. For small fiscal units, a marginal analysis approach will be used. Present expansion capacity of the various munizipal services can be estimated and incremental costs of expanding those services can be calculated. These figures may then be used to predict municipal Elscal costs for each service for some future period.

As a first step torard such an analysis, a basic description of the four najor municipalitics (Brabley, Calexico, E1 Centro, and Holtville) in Imperial County was obtained from unpub].ished data. 11

\section{Brawley}

Brawley urban water supplics aro obtained from IID by the Brawley County Water District. Current (late 1975) average daily consumption for Brawley is approzimately 6.5 million gal. The municipal district has a 10-da-storage reserve, pretreatment settling basins and facilities for chemical mixing, filtration, and chlorination. The 11pump distribution system has a capacity of $276,000 \mathrm{ga} / \mathrm{min}$ and has: a minimum pipe size of $4 \mathrm{in}$. Electricity is supplied throughout Brawley exclusively by IID and natural gas by the Southern California Gas Company. There is one firc station in Brawley staffed by 19 men and several additional reserve personnel. The city has recelved a class four insurance rating but a recent report in the Imperial Valley 
Press (May 12, 1976) states that this rating will be changed to a class five unless substantial updating of the fire department is undertaken. There are currently 20 sworn Brawley policemen and 7 nonsworn staff personnel. The city currently maintains 102 acres of park area distributed among 9 park sites. The largest of these, New River Park, is 66.6 acres with all remaining parks below 10 acres in size. The Pioneers Memorial Hospital, part of a special district local government, is partially tax supported and provides a 78-bed capacity for the Brawley area. In addition the Clinica de Salubridad de Campesinos Clinic in Brawley, for migrant workers, serves between 75 to 100 per day. Finally, the city provides solid waste disposal service throughout Brawley. Refuse is transferred to a county sanitary landfil1.

\section{Calexico}

Water and sewer service in Calexico are supplied through the municipality. System capacity is 15.4 million gpd. City calculations for 1975 indicate an average consumption of 2.75 million gpd. Eletricity and gas are supplied in Calexico, as in Brawley, by IID and Southern California Gas Company, respectively. The city fire department employ's 2 full-time and 18 part-time firemen with 11 reserve volunteers, all based from one central station. The department has 5 trucks, the largest of which has a $500 \mathrm{gpm}$ capacity. The pólice department employs 24 sworn officers and has 7 patrol cars all based in 1 central facility. There are seven parks within city limits all of which axe city owned and maintained. Total park acreage has not yet been obtained. There is a 34-bed-capacity hospital in Calexico with a professional staff of 12 doctors.

\section{EI Centro}

E1 Centro residents are provided with water and sewer services by the city. The city also operates solid waste disposal service. Gas and electricity service is provided privately as in Brawley and Calexico. The EI Centro fire department has 9 trucks, 30 full-time firemen and maintains 2 stations. The department is also under contract with the county to protect county areas surrounding E1 Centro. There are 35 sworn officers in the E1 Centro police department and 15 patrol cars. Only one station is maintained and all activities are centrally located in that facility. The city of $E 1$ Centro maintains 54 acres of park within city boundaries. Twenty acres are located in Bucklin Park with the remaining 34 acres scattered throughout the city as small neighborhood 
parks. The EI Centro Community Hospital with a capacity of 92 beds, and the Valley Convalescent Hospital with a capacity of 120 beds serve El Centro for health services. EI Centro also has a central city public library.

\section{Holtville}

Water and sewer service within city limits are provided by the city; however, system capacity and treatment data have not yet been obtained. Solid waste disposal service is operated by the city. The city fire department has three cityowned trucks and one county-orned truck available for emergency use, a11 located in one station house. There are 10 sworn police officers and 1 nonsworn full-time employee. Holtville has four parks within city limits, two of which (including Angels Park, the spring training camp for the California Angels) are dedicated to baseball fields. These parks comprise 12.6 acres or 2.1\% of Holtville city area. There is a small county operated public library in Holtville.

The relationship between city boundaries and KGRAs is given in
Fig. 7.6 with overlapping areas darkened. Since current county regulations prohibit geothermal development within city boundaries, these overlap areas represent additional geothermal exclusion zones within KGRAs. However, the proximity of each city to the various licRls is still very important since nearby communities will be strongly affected by population influxes and general economic activity associated with geothermal development on each IGRA.

Federal Governmenta1 Influence Hithin the County

Table 7.6 lists direct county fiscal revenues from federal agencles. However, the total dollar expenditure of the federal government within the county through federal projects, payrolls, other than direct county subsidies, etc., is much larger than the $\$ 9+$ million shown in Table 7.6. Table 7.11 shows Imperial County calculations of the total direct economic influence of the various: federal agencies. These total expendture figures make the fedcral government the second largest industcy in the county, surpassed only byr agriculture. 
Table 7.11. Federal government expenditures in Imperial County. 10

Federal agency

Expenditures

(thousands of dollars)

$1970 \mathrm{~F} . \mathrm{Y}$.

1972 F.Y.

1. Department of Health, Education and Welfare

2. Department of Agriculture

3. Department of Defense

4. Department of Transportation

5. General Services Administration

6. Department of Justice

7. Treasury Department

8. Department of Interior

9. Civil Service Commission

10. Department of Labor

other

$\begin{array}{rr}22,450 & 30,345 \\ 20,738 & 16,031 \\ 7,969 & 10,722 \\ 11,544 & 9,197 \\ 362 & 5,370 \\ 2,972 & 3,891 \\ 3,649 & 3,446 \\ 4,761 & 2,013 \\ 1,201 & 1,666 \\ 404 & 1,359 \\ 5,028 & 3,706\end{array}$

Total

81,078

87,746 


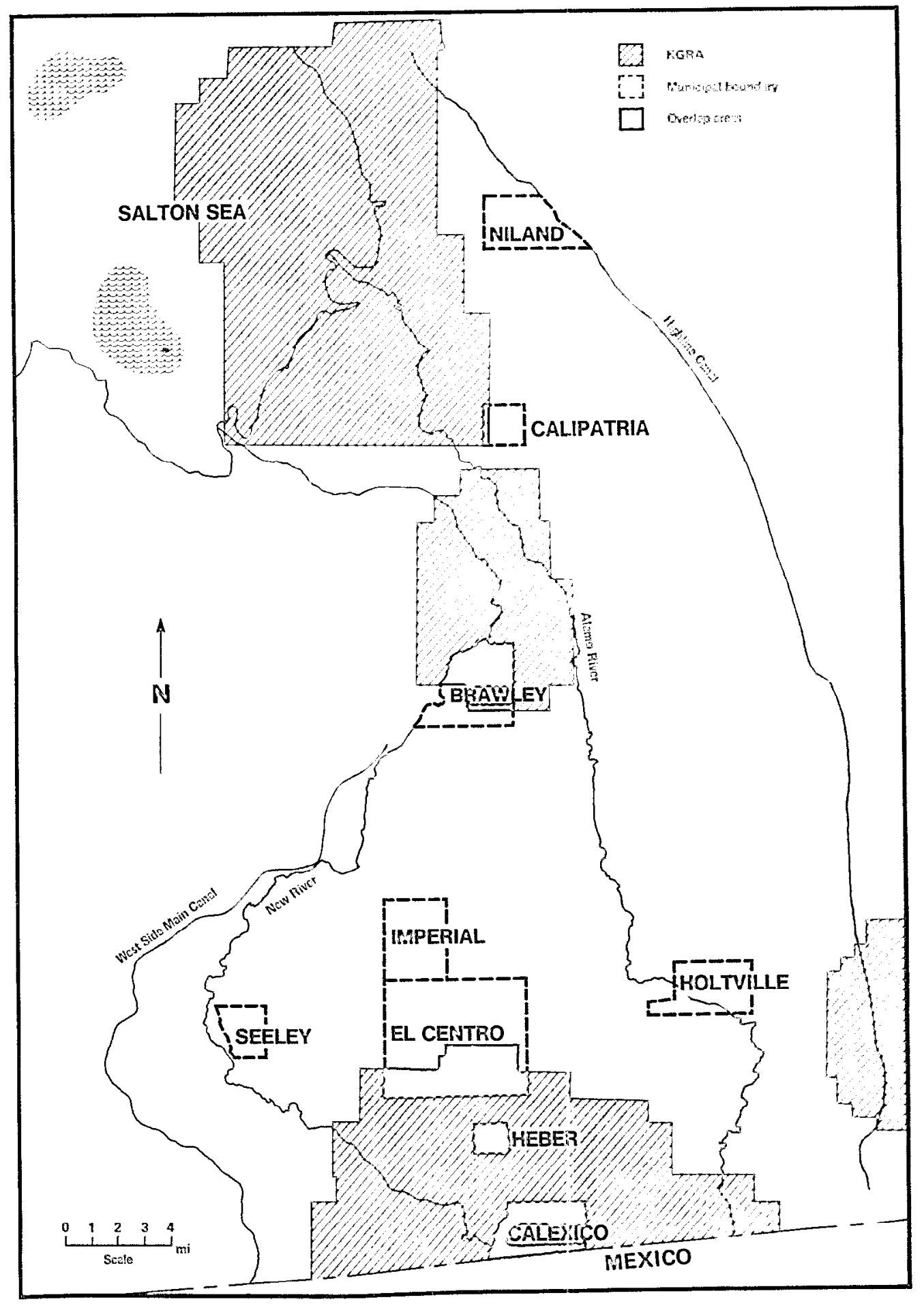

Fig. 7.6. Imperial Valley city boundary overlap with liGRAs. 


\section{REFERENCES}

1. A. M. Alexander, County Administrative Officer. 1975-76 Final Budget and Work Program, Imperial County California, September 1975.

2. California State Board of Equalization, Tax-Rate Area Map, Imperial County (Sept. 10, 1975).

3. Office of the Auditor-Controller, Imperial County, County of Imperial Tax Rates and Information, Fiscal Year 1975-76.

4. Office of the Auditor-Controller, Imperial County, County of Imperial Tax Rates and Information, Fiscal Year 1973-74.

5. Office of the Auditor-Controller, Imperial County, County of Imperial Tax Rates and Information, Fiscal Year 1974-75.

6. "Mistake Leaves County Budget $\$ 900,000$ Short," Imperial ValZey Press. Vol 76-No. 13 (May 12, 1976).

7. Office of the Imperial County Superintendent of Schools, 23rd Annual Financial and Statistical Report 1974-1975 (November 1975).

8. Imperial County Department of Public Works, Terms, Condition, Standards, and Application Procedures For Initial Geothermal Development Imperial County (May 1971).

9. Imperial County Planning Department. Current Land Use PLan - Northem and Southern Planning Area, Imperial Valley (1973).

10. Imperial County Planning Department, Imperial County ULtimate Land Use Plan (June 25, 1973).

11. P. Renaldo, Imperial County Social-Economic Characterization. Unpublished data report for Campesinos Unidos (February 1976). 


\section{Section 8 \\ Social Characterization of Imperial county}

Kenda11 Haven

\subsection{IMPERIAL COUNTY SOCIOLOGICAL DESCRIPTION}

The primary informational source for this section is the 1970 county level census data. ${ }^{1}$ Portions of this data are updated annually by various state and local agencies with most of this work being done by the State Department of Finance. However, these are estimates based on 1970 census data based on historical trends. These estimates are made for only a small percentage of the total number of parameters calculated by the census survey. As a result, a more reliable, complete social composite can be created from 6-year-old census data than from the available updated estimates. Our study is based on 1970 census survey data augmented where appropriate with updated information. Five groups of the census data were selected for use in this characterization: distribution by age, distribution by racial groups, educational attainment, and income level, and two poverty indicators. A summary of this data for Imperial County is presented in Tables 8.1 through 8.4 .

Data presented in Tables 8.1 through 8.4 were also collected to compare all California counties.
Location quotients were calculated for each parameter to determine if Imperial County social distribution was similar to that of other counties. (See Tables 8.5 and 8.8).

The age distribution shown in Table 8.1 is somewhat unusual. The population of each group may be divided by the age span of that group to obtain an estimate of the number of people of a given age. These results are plotted in Fir. 8.1 Comparing these figures with data fresented in Table 8.5 , we find that Imperial County has a higher concentration of both children and young teenagers than any other county in the state, yot it rarks 17 th from the bottom for concentration of 18 to 24 ycar old group. This indicates two things: First, a substantial post high school migration out of the county, and second, a high average number of dependent children per family. The high number of dependent children per family may be translated into a high fertility rate for the county. This phenomenon has also been noted and reported by Picl. ${ }^{2}$ In adclition, Imperial County is relatively low in Black, Japanese, and Chinese populations, but, as 
Table 8.1. Imperial County population distribution by age group (1970). 1

\begin{tabular}{rcrr}
\hline Age group & $\begin{array}{c}\text { Number of county } \\
\text { persons in group }\end{array}$ & $\begin{array}{c}\text { \% Total } \\
\text { county population }\end{array}$ & Cumulative \% \\
\hline 0 through 13 & 23,918 & 32.1 & 32.1 \\
14 through 17 & 7251 & 9.8 & 41.9 \\
18 through 24 & 7072 & 9.5 & 51.4 \\
25 through 34 & 8363 & 11.2 & 62.6 \\
35 through 44 & 8368 & 11.2 & 73.8 \\
45 through 54 & 7682 & 10.3 & 84.1 \\
55 through 64 & 6245 & 8.4 & 92.5 \\
over 64 & 5575 & 7.5 & 100.0 \\
Total & 74,492 & $100 \%$ &
\end{tabular}

Table 8.2. Imperial County population distribution by racial group (1970)..$^{1}$

\begin{tabular}{lrc}
\hline \multicolumn{1}{c}{ Racial group } & $\begin{array}{r}\text { Number of persons } \\
\text { in group }\end{array}$ & $\%$ of Total county population \\
\hline White & 68,806 & 92.4 \\
Black & 2,586 & 3.5 \\
American Indian & 889 & 1.2 \\
Japanese & 206 & 0.3 \\
Chinese & 412 & 0.6 \\
Filipino & 731 & 1.0 \\
Other & 862 & 1.2 \\
Total & 74,492 & 100.2 \\
Spanish American & 34,260 & 46.0 \\
(4th count data) & &
\end{tabular}


Table 8.3. Imperial County educational attainnent for persons 25 years and older (1970).1

Highest grade completed

Number of county persons

$\%$ Total (25 yrs and older)

\begin{tabular}{lrc}
\hline Below grade 8 & 10,305 & 28.4 \\
Grade 8 & 4,152 & 11.4 \\
Grades $9-11$ & 6,179 & 17.1 \\
Grade 12 & 8,924 & 24.6 \\
Grades $13-15$ & 4,029 & 11.1 \\
Grade 16 and over & 2,662 & 7.3 \\
Total & 36,251 & 99.9 \\
\hline
\end{tabular}

Table 8.4. Imperial County income and poverty indicators (1970). 1

\begin{tabular}{|c|c|c|c|c|}
\hline Annual income & $\begin{array}{l}\text { Number of } \\
\text { county } \\
\text { families }\end{array}$ & $\begin{array}{l}\% \text { Total } \\
\text { number of } \\
\text { families }\end{array}$ & $\begin{array}{l}\text { Number of } \\
\text { county } \\
\text { unrelated } \\
\text { individuals }\end{array}$ & $\begin{array}{l}\% \text { Total } \\
\text { number of } \\
\text { individuals }\end{array}$ \\
\hline Less than $\$ 4,000$ & 3,086 & 17.9 & 3,256 & 68.9 \\
\hline$\$ 4,000-\$ 6,000$ & 2,588 & 15.0 & 548 & 11.6 \\
\hline$\$ 6,000-\$ 8,000$ & 2,630 & 15.2 & 377 & 8.0 \\
\hline$\$ 8,000-\$ 10,000$ & 2,316 & 13.4 & 243 & 5.1 \\
\hline$\$ 10,000-\$ 15,000$ & 3,952 & 22.9 & 203 & 4.3 \\
\hline$\$ 15,000-\$ 25,000$ & 2,102 & 12.2 & $101^{\mathrm{a}}$ & 2.1 \\
\hline Over $\$ 25,000$ & 587 & 3.4 & & \\
\hline Total & 17,261 & 100.0 & 4,728 & 100.0 \\
\hline $\begin{array}{l}\text { Number below } \\
\text { poverty level }\end{array}$ & 2,781 & 16.1 & 1,692 & 35.8 \\
\hline
\end{tabular}

${ }^{a}$ Includes all unrelated individuals with incone over $\$ 15,000$ annually. 
Table 8.5. Age distribution location quotients for California counties.

\begin{tabular}{|c|c|c|c|c|c|c|c|c|c|c|c|}
\hline 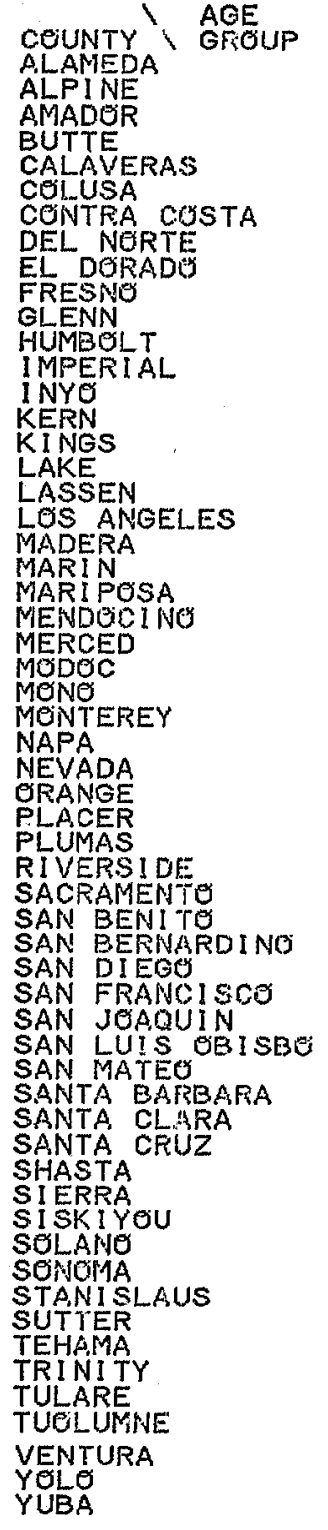 & 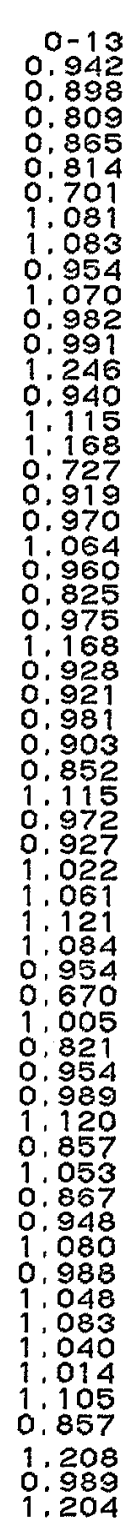 & 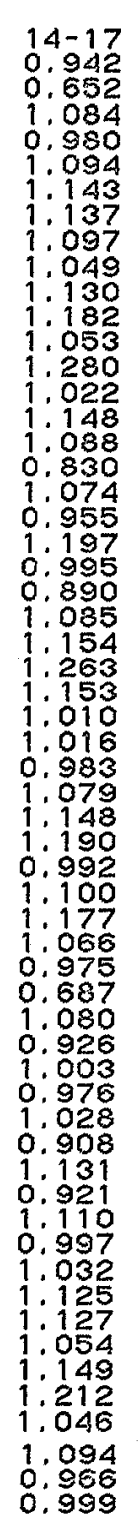 & 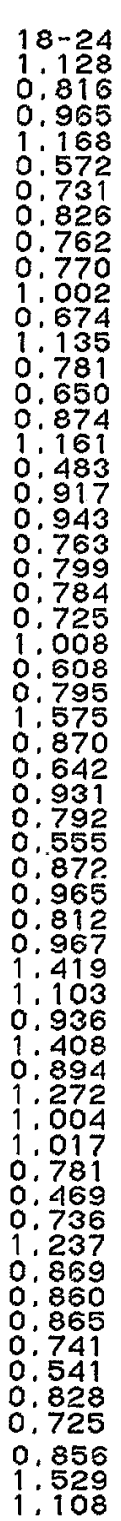 & 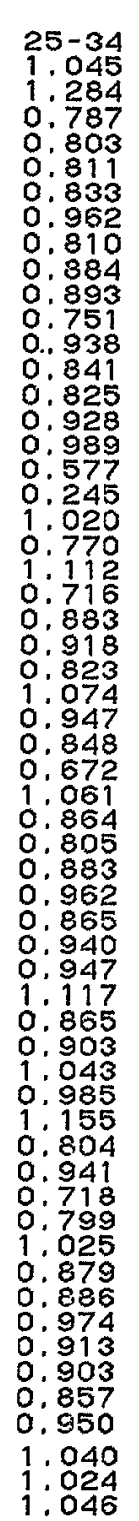 & 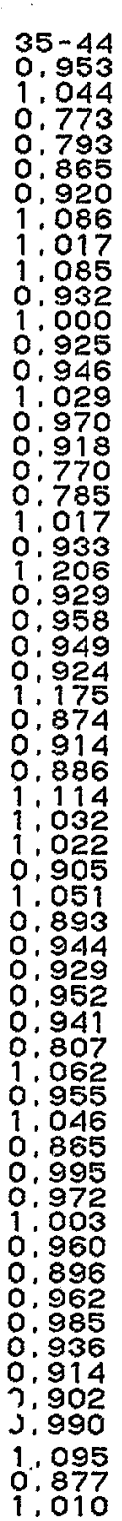 & 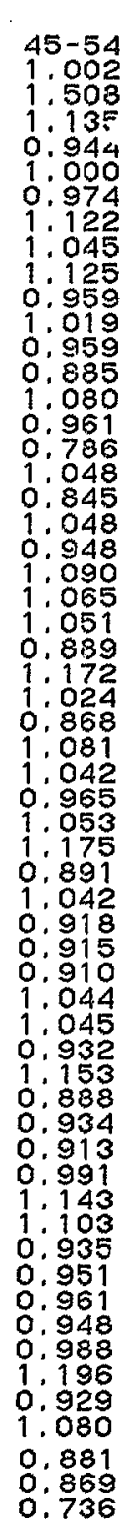 & 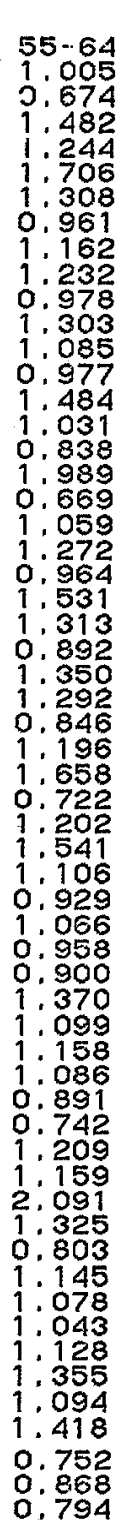 & 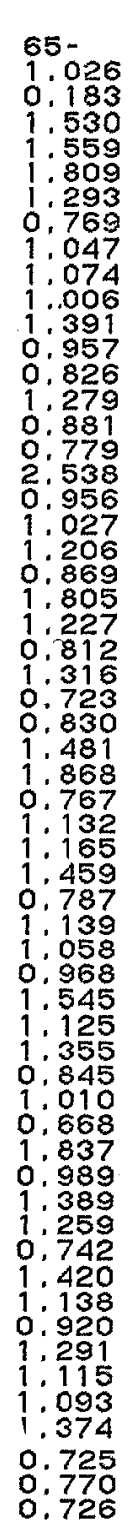 & 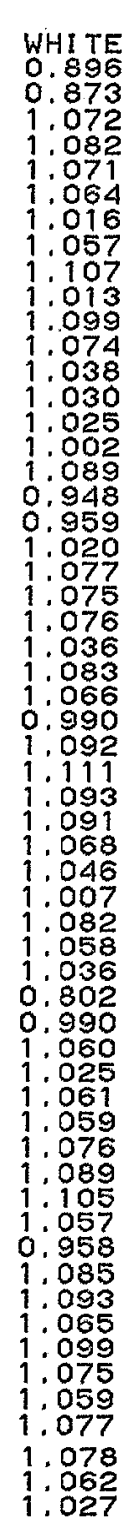 & 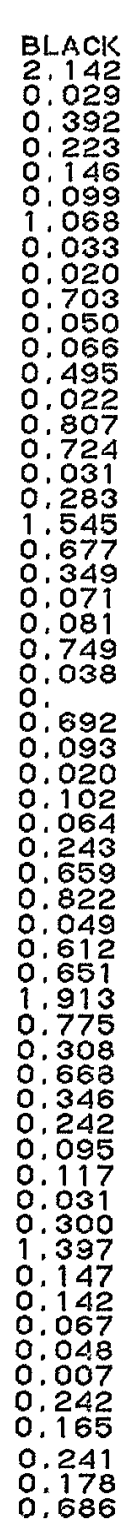 & 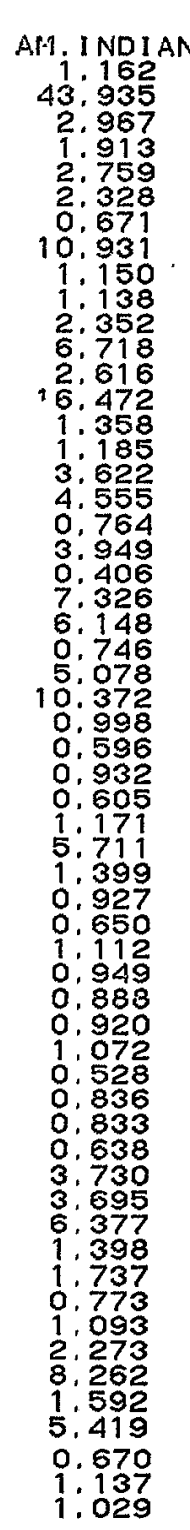 \\
\hline
\end{tabular}


Table 8.6. Racial group distribution location quotients for California counties (1970).

\begin{tabular}{|c|c|c|c|c|c|c|c|c|c|c|}
\hline 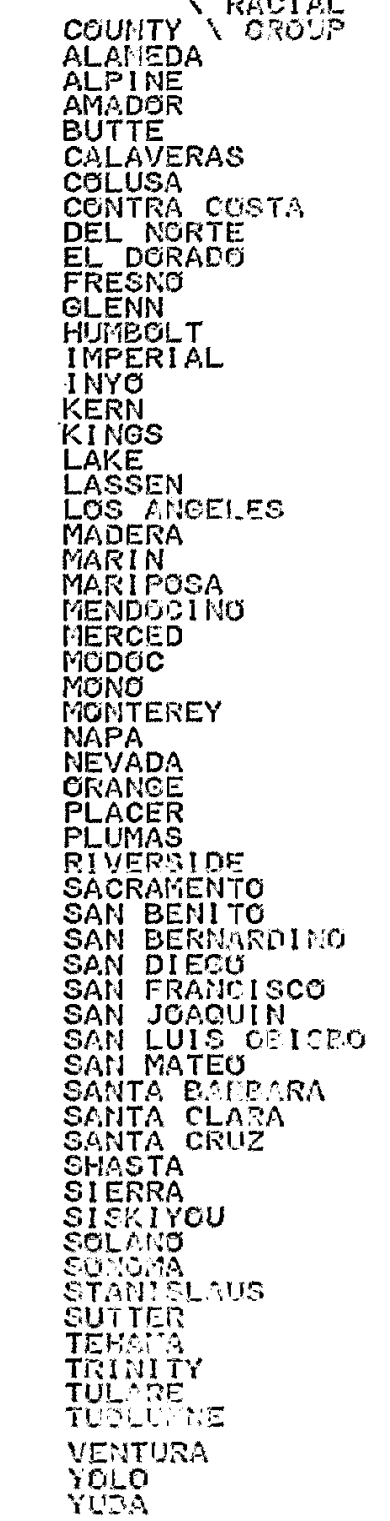 & 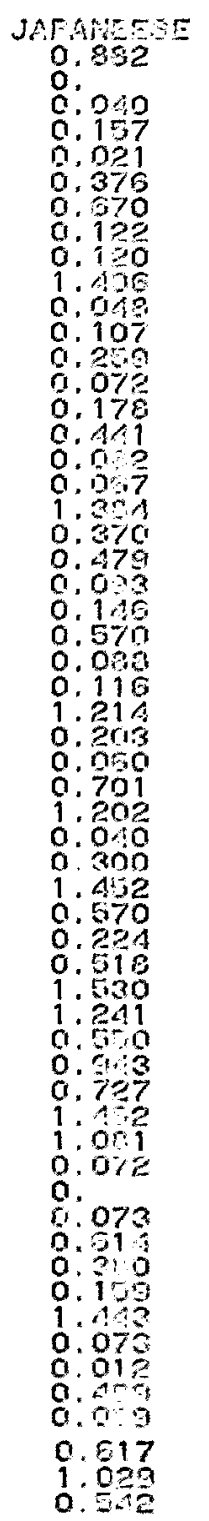 & 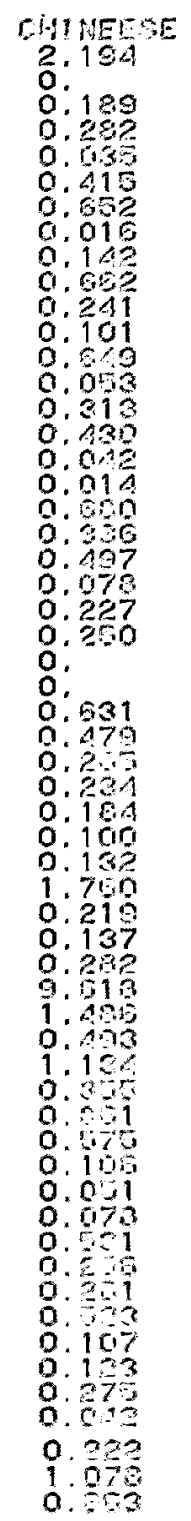 & 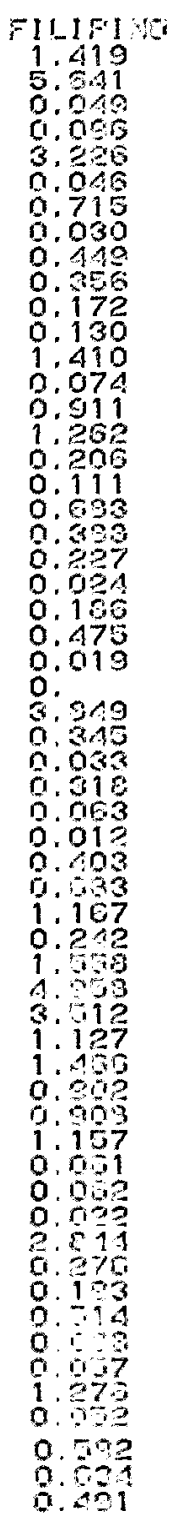 & 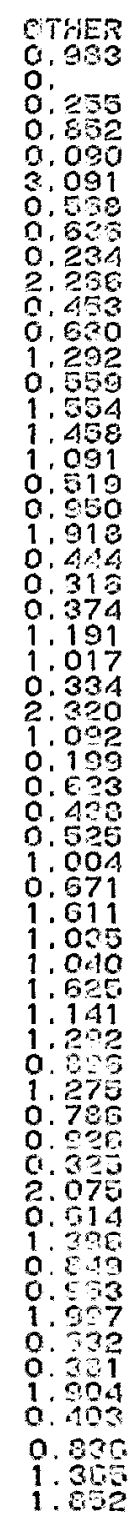 & 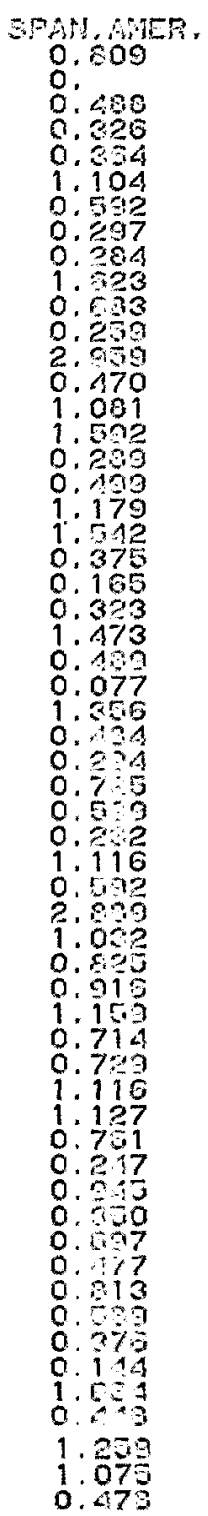 & 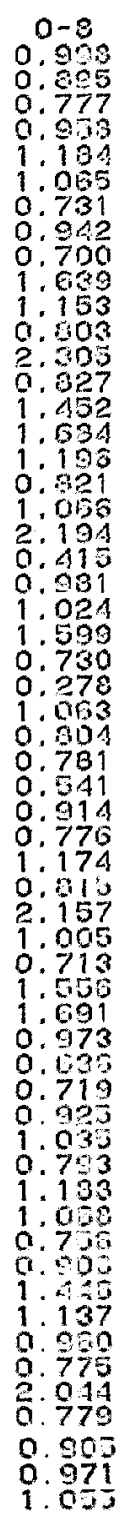 & 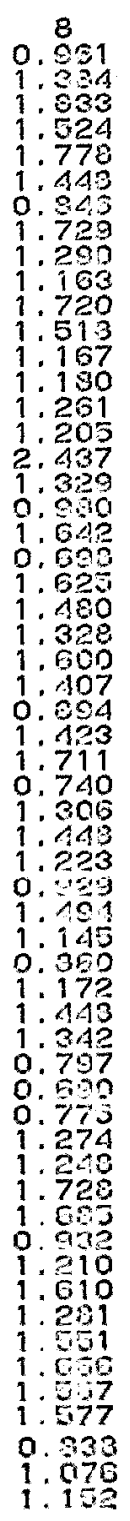 & 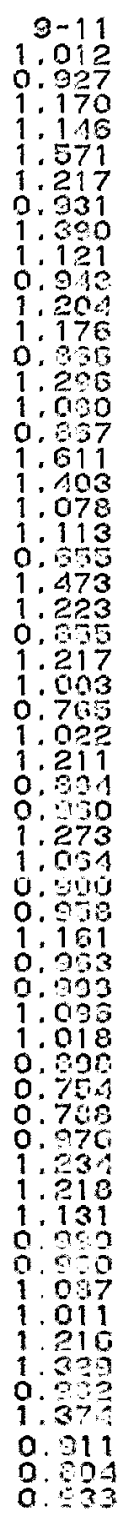 & 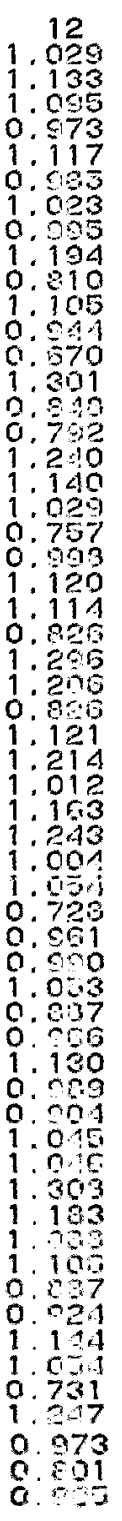 & 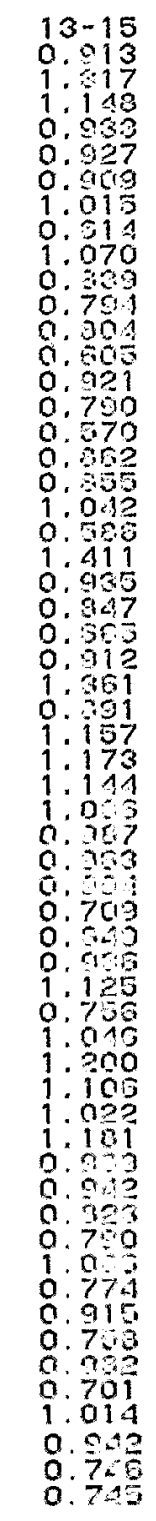 \\
\hline
\end{tabular}


Table 8.7. Educational attainment location quotients for California counties (1970).

\begin{tabular}{|c|c|c|c|c|c|c|}
\hline \multirow[b]{2}{*}{ County } & \multicolumn{3}{|c|}{$\begin{array}{l}\text { Highest grade } \\
\text { attained }\end{array}$} & \multirow[b]{2}{*}{12} & \multirow[b]{2}{*}{$13-15$} & \multirow{2}{*}{$\begin{array}{c}16 \text { and } \\
\text { over }\end{array}$} \\
\hline & $<8$ & 8 & $9-11$ & & & \\
\hline Alameda & 0.998 & 0.961 & 1.012 & 1.029 & 0.913 & 1.094 \\
\hline Alpine & 0.895 & 1.384 & 0.927 & 1.133 & 1.317 & 0.226 \\
\hline Amador & 0.777 & 1.833 & 1.170 & 1.095 & 1.148 & 0.685 \\
\hline Butte & 0.958 & 1.524 & 1.146 & 0.973 & 0.933 & 0.843 \\
\hline Calaveras & 1.184 & 1.778 & 1.571 & 1.117 & 0.927 & 0.654 \\
\hline Colusa & 1.065 & 1.448 & 1.217 & 0.985 & 0.909 & 0.765 \\
\hline Contra Costa & 0.731 & 0.846 & 0.931 & 1.023 & 1.015 & 1.269 \\
\hline De1 Norte & 0.942 & 1.729 & 1.390 & 0.995 & 0.614 & 0.546 \\
\hline E1 Dorado & 0.700 & 1.290 & 1.121 & 1.194 & 1.070 & 0.828 \\
\hline Fresno & 1.639 & 1.163 & 0.943 & 0.810 & 0.839 & 0.717 \\
\hline Glenn & 1.153 & 1.720 & 1.204 & 1.105 & 0.794 & 0.548 \\
\hline Humboldt & 0.803 & 1.518 & 1.176 & 0.944 & 0.804 & 0.716 \\
\hline Imperial & 2.305 & 1.167 & 0.866 & 0.670 & 0.605 & 0.488 \\
\hline Inyo & 0.827 & 1.180 & 1.296 & 1.301 & 0.921 & 0.764 \\
\hline Kern & 1.452 & 1.261 & 1.080 & 0.848 & 0.790 & 0.631 \\
\hline Kings & 1.684 & 1.205 & 0.867 & 0.792 & 0.570 & 0.555 \\
\hline Lake & 1.196 & 2.437 & 1.611 & 1.240 & 0.862 & 0.676 \\
\hline Lassen & 0.821 & 1.329 & 1.403 & 1.140 & 0.855 & 0.583 \\
\hline Los Angeles & 1.066 & 0.980 & 1.078 & 1.029 & 1.042 & 0.978 \\
\hline Madera & 2.194 & 1.642 & 1.113 & 0.757 & 0.586 & 0.517 \\
\hline Marin & 0.415 & 0.698 & 0.655 & 0.998 & 1.411 & 2.106 \\
\hline Mariposa & 0.981 & 1.625 & 1.473 & 1.120 & 0.935 & 0.862 \\
\hline Mendocino & 1.024 & 1.480 & 1.223 & 1.114 & 0.847 & 0.735 \\
\hline Merced & 1.599 & 1.328 & 0.855 & 0.826 & 0.665 & 0.553 \\
\hline Modoc & 0.730 & 1.600 & 1.217 & 1.296 & 0.912 & 0.561 \\
\hline Mono & 0.278 & 1.407 & 1.003 & 1.206 & 1.361 & 0.832 \\
\hline Monterey & 1.063 & 0.894 & 0.765 & 0.826 & 0.891 & 0.981 \\
\hline Napa & 0.804 & 1.423 & 1.022 & 1.121 & 1.157 & 0.906 \\
\hline Nevada & 0.781 & 1.711 & 1.211 & 1.214 & 1.173 & 0.835 \\
\hline Orange & 0.541 & 0.740 & 0.884 & 1.012 & 1.144 & 1.119 \\
\hline Placer & 0.914 & 1.306 & 0.960 & 1.163 & 1.036 & 0.770 \\
\hline Plumas & 0.776 & 1.448 & 1.273 & 1.243 & 0.987 & 0.749 \\
\hline Riverside & 1.174 & 1.223 & 1.064 & 1.004 & 0.963 & 0.805 \\
\hline Sacramento & 0.815 & 0.929 & 0.900 & 1.054 & 0.994 & 0.939 \\
\hline San Benito & 2.157 & 1.494 & 0.958 & 0.728 & 0.709 & 0.462 \\
\hline San Bernardino & 1.005 & 1.145 & 1.161 & 0.961 & 0.849 & 0.657 \\
\hline San Diego & 0.713 & 0.860 & 0.963 & 0.990 & 0.936 & 0.967 \\
\hline San Francisco & 1.556 & 1.172 & 0.998 & 1.053 & 1.125 & 1.461 \\
\hline San Joaquin & 1.691 & 1.448 & 1.096 & 0.887 & 0.756 & 0.593 \\
\hline San Luis Obisbo & 0.973 & 1.342 & 1.018 & 0.966 & 1.046 & 0.831 \\
\hline San Mateo & 0.636 & 0.797 & 0.898 & 1.130 & 1.200 & 1.331 \\
\hline Santa Barbara & 0.719 & 0.690 & 0.754 & 0.989 & 1.106 & 1.261 \\
\hline Santa Clara & 0.925 & 0.775 & 0.788 & 0.904 & 1.022 & 1.359 \\
\hline Santa Cruz & 1.035 & 1.274 & 0.976 & 1.045 & 1.181 & 1.059 \\
\hline Shasta & 0.793 & 1.248 & 1.234 & 1.046 & 0.989 & 0.644 \\
\hline Sierra & 1.188 & 1.728 & 1.218 & 1.308 & 0.942 & 0.831 \\
\hline
\end{tabular}


Table 8.7. (Concluded)

\begin{tabular}{lllllll}
\hline Siskiyou & 1.068 & 1.685 & 1.131 & 1.183 & 0.823 & 0.599 \\
Solano & 0.756 & 0.932 & 0.990 & 1.088 & 0.790 & 0.626 \\
Sonoma & 0.908 & 1.210 & 0.960 & 1.106 & 1.086 & 0.850 \\
Stanislaus & 1.446 & 1.610 & 1.087 & 0.887 & 0.774 & 0.605 \\
Sutter & 1.137 & 1.281 & 1.011 & 0.924 & 0.915 & 0.776 \\
Tehama & 0.960 & 1.551 & 1.216 & 1.144 & 0.758 & 0.564 \\
Trinity & 0.775 & 1.666 & 1.329 & 1.054 & 0.982 & 0.762 \\
Tulare & 2.044 & 1.567 & 0.982 & 0.731 & 0.701 & 0.513 \\
Tuolumne & 0.779 & 1.577 & 1.374 & 1.247 & 1.014 & 0.669 \\
Ventura & 0.905 & 0.838 & 0.911 & 0.973 & 0.942 & 0.844 \\
Yolo & 0.971 & 1.076 & 0.804 & 0.801 & 0.746 & 1.223 \\
Yuba & 1.055 & 1.152 & 0.938 & 0.925 & 0.745 & 0.533 \\
& & & & & & \\
\hline
\end{tabular}

would be expected, is very high in Spanish-Americans (see Table 8.6). The county ranks fairly low in adult educational attainment (see Table 8.7) with the highest concentration of adults with less than an eighth grade education, an average concentration of adults with an eighth grade education, and steadily decreasing rankings for higher educational.

levels. This educational profile can be related to the characteristics of employment and agricultural patterns in the county. For example, both Fresno and Kern counties (two other agricultural1y based counties using large quantities of seasonal labor) have very similar educational attainment profiles.

Table 8.8, shows that Imperial County family income distribution is well within the normal ranges for California counties. The unrelated individual section, however, points out that a11 location quotients in this section for Imperial County are low. This means that unrelated individuals, as a group, represent a much smaller part of the Imperial County population than that group represents for the state as a whole and is add $1-$ tional evidence of the county's unrelated young outmigration. For those unrelated individuals who do remain in Imperial County, the income distribution is fairly typical.

In fact, the relative drop off in the number of unrelated individuals making over $\$ 15,000$ per year is smaller in Imperial County than in many other California countics. Imperial County has high poverty level indicators for both families and unrelated individuals (the 0.805 location quotient for the poverty indicator for unrelated individuals must be weighed against the relatively low number of unrelated individuals 
Table 8.8. Income and poverty indicator location quotients for California counties.

\begin{tabular}{|c|c|c|c|c|c|c|c|c|c|c|c|}
\hline \multicolumn{9}{|c|}{ FAMILIES } & \multicolumn{3}{|c|}{ UNRELATED INOIVIDUALS } \\
\hline 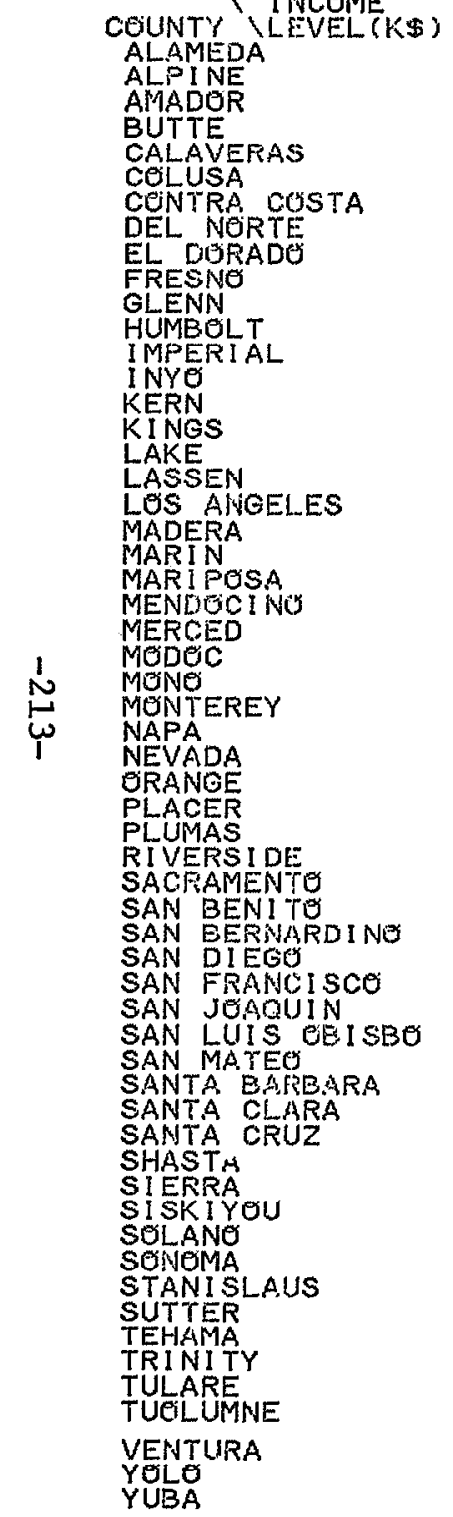 & 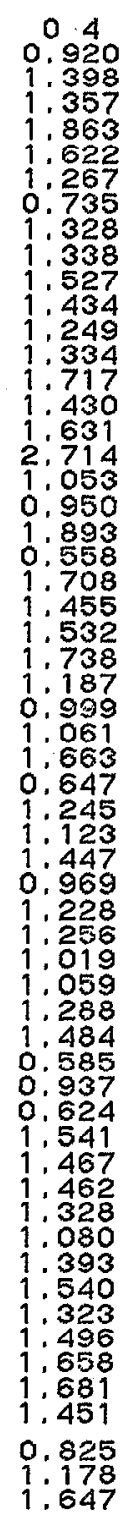 & 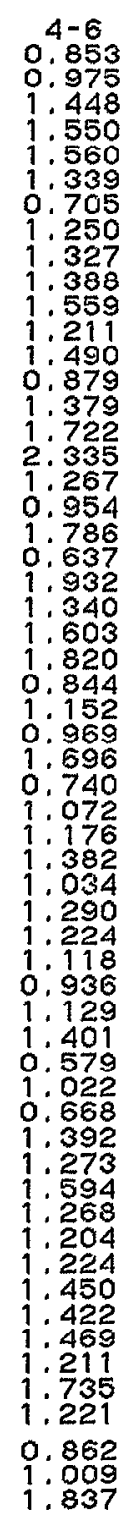 & 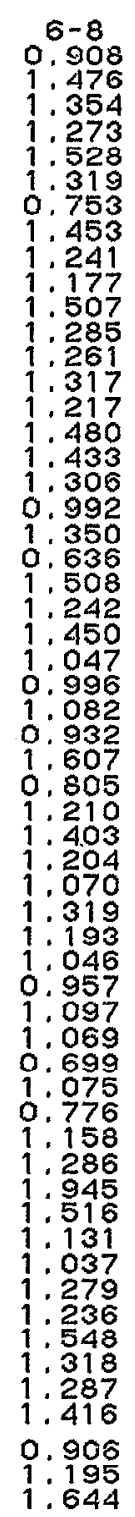 & 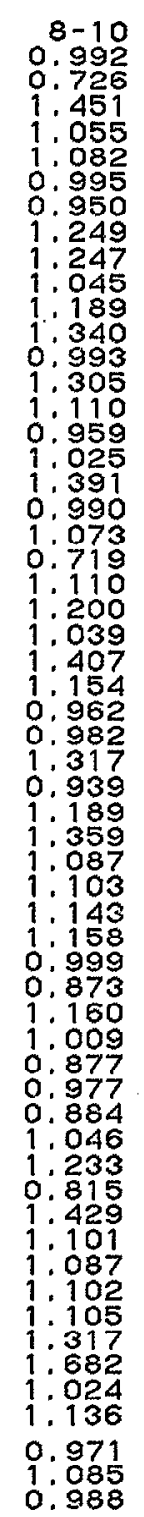 & 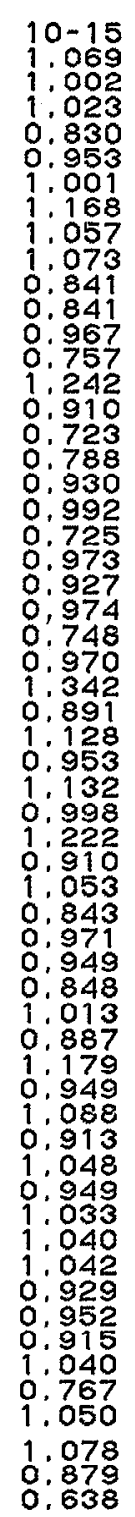 & 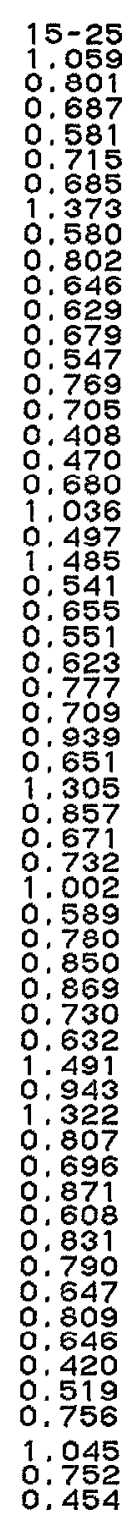 & 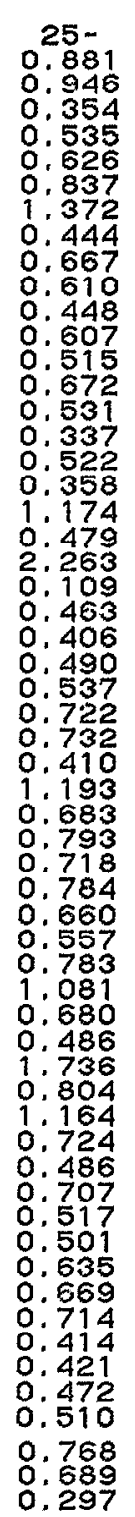 & 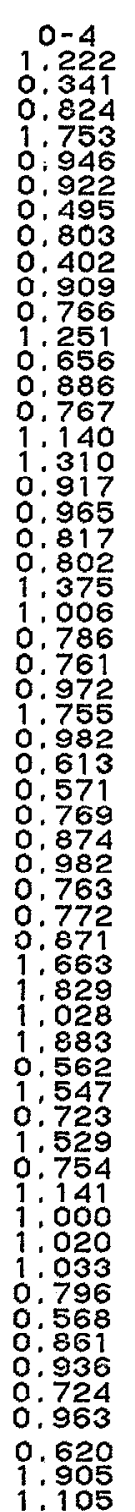 & 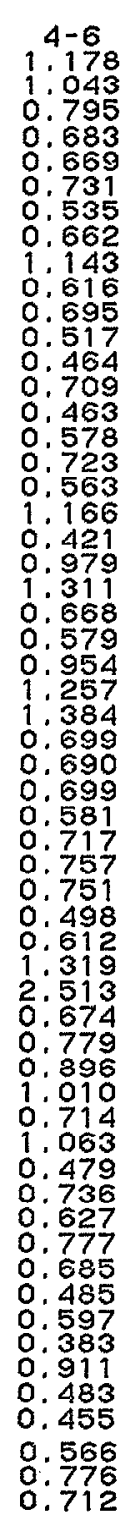 & 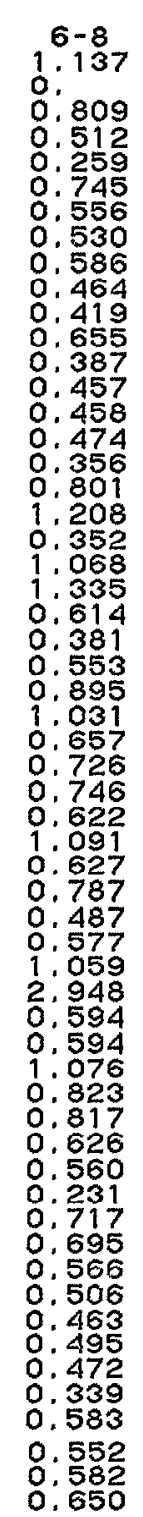 & 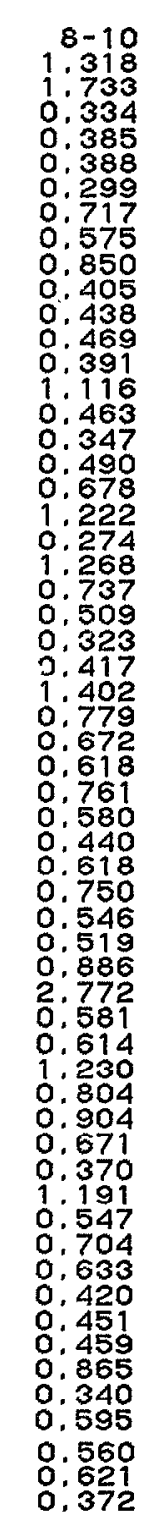 \\
\hline
\end{tabular}


Table 8.8. (Concluded)

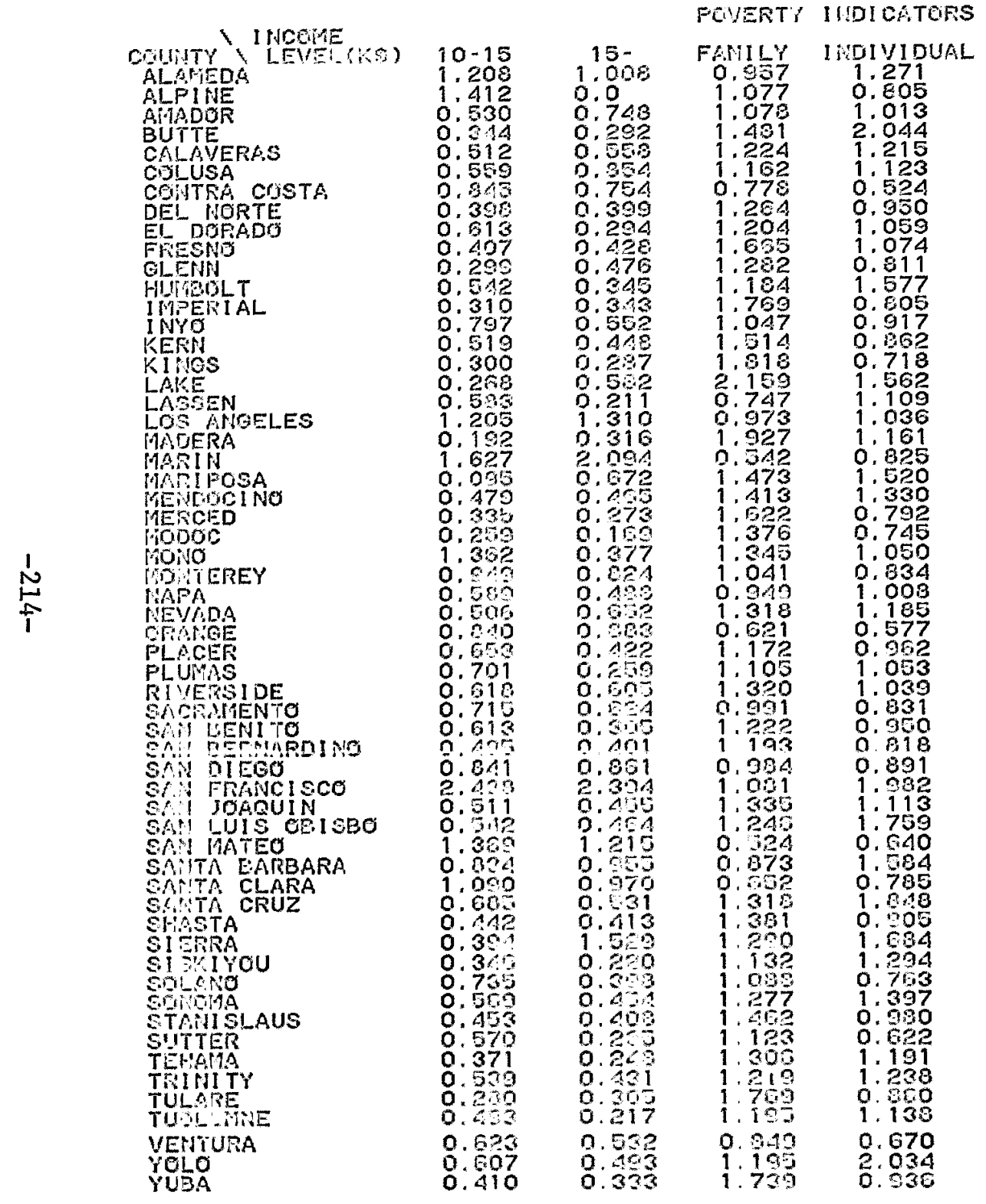


in the county), but such statistics must be viewed in light of the large number of migrant and seasonal farm workers employed in Imperial county. Most of the poverty is concentrated in this group rather than spread across a large number of job classes. Poverty is a problem in Imperial County, but it is certainly not a unique problem to the county in either its existence or magnitude.

\subsection{INTRACOUNTY SOCIOLOGICAL DESCRIPTION}

\section{INTRACOUNTY POPULATIONS}

Census survey data are available for urban populations every 10 years. Census data also covers some unincorporated areas with populations over 1000 and populations of several rural county census divisions. However these figures do not include specific populations of any of Imperial County's smaller and unincorporated communities. Some estimates for these populations have been obtained from personal interviews, from data from the State Department of Finance, and from basic census data interpolation. The resultant data from all of these sources are shown in Table 8.9 A11 areas listed below Calipatria are estimates, whereas all data for Calipatria and above represent official census or State Department of Finance data. The term, total municipal population, indicates the - total population living in all

incorporated and unincorporated towns. Rural populations are those outside town boundaries. Total Imperial Valley population was obtained by including appropriate rural county census divisions for 1960 and 1970 with Imperial Valley municipal populations. The area of most rapid increase within the county in recent years has been the Imperial-Calexico axis. Note that urban related populations are increasing relative to rural populations. Both the percentage of county populations and number of individuals representing rural population are decreasing.

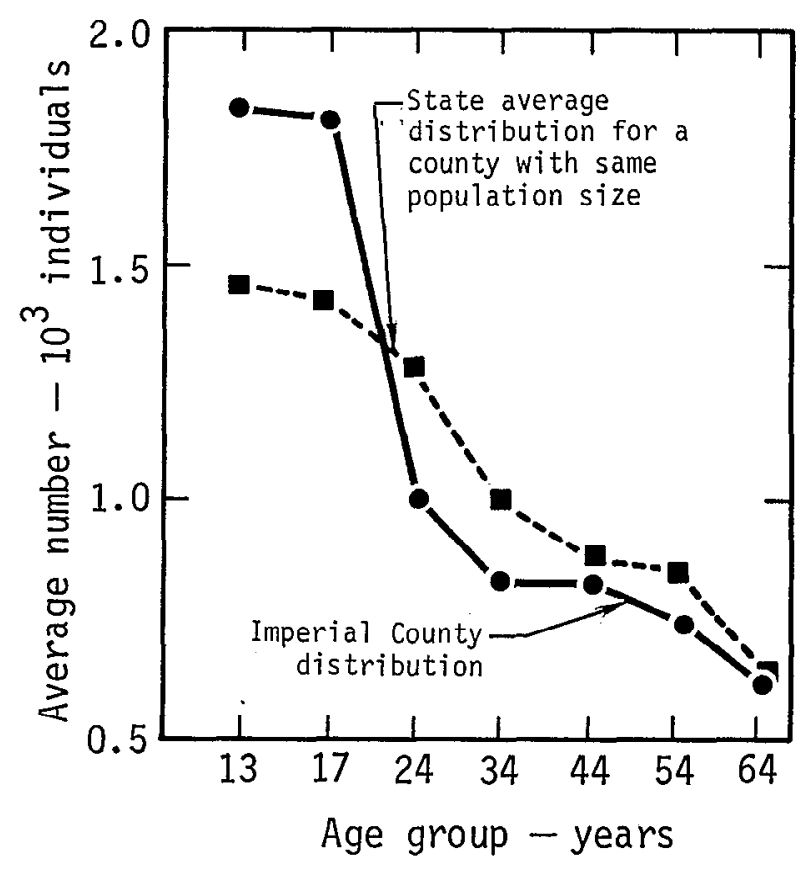

Fig. 8.1. Imperial County population distribution as a function of age. Numbers in the ordinate indicate maximum age in group, i.e., 34 means ages 24 to 34.1 
Table 8.9. Imperial County intracounty population distribution 1950 to 1974.1

\begin{tabular}{|c|c|c|c|c|c|}
\hline Community & 1950 & 1960 & $\begin{array}{c}\text { Population } \\
1970\end{array}$ & 1974 & $\begin{array}{l}\text { Change } \\
1950-1974\end{array}$ \\
\hline E1 Centro & 12,590 & 16,811 & 19,272 & 21,300 & +69.2 \\
\hline Imperial & 1,759 & 2,658 & 3,094 & 3,210 & +82.5 \\
\hline Calexico & 6,443 & 7,992 & 10,625 & 12,829 & +99.1 \\
\hline Brawley & 11,922 & 12,703 & 13,746 & 13,940 & +16.9 \\
\hline Holtville & 2,472 & 3,080 & 3,496 & 3,580 & +44.8 \\
\hline Westmorland & 1,010 & 1,404 & 1,175 & 1,407 & +39.3 \\
\hline Calipatria & 1,428 & 1,848 & 1,824 & 1,890 & +32.4 \\
\hline Niland & & 900 & 1,050 & 1,137 & \\
\hline Heber & & 1,700 & 1,850 & 2,206 & \\
\hline Seeley & & 700 & 900 & 1,024 & \\
\hline Plaster City & & 50 & 80 & 86 & \\
\hline $0 \operatorname{cotillo}$ & & 200 & 200 & 233 & \\
\hline Palo Verde & & 300 & 350 & 440 & \\
\hline Winterhaven-Band & & 1,973 & 2,060 & 2,142 & \\
\hline Other non valley rural & & 1,551 & 1,157 & 1,430 & \\
\hline Imperial valley rural & & 18,235 & 13,613 & 16,822 & \\
\hline $\begin{array}{l}\text { Total municipa1 } \\
\text { population }\end{array}$ & & 52,319 & 59,722 & 65,424 & \\
\hline $\begin{array}{l}\text { Total county } \\
\text { population }\end{array}$ & 62,516 & 72,105 & 74,492 & 83,676 & +33.9 \\
\hline $\begin{array}{l}\text { Total rural } \\
\text { population }\end{array}$ & & 19,786 & 14,770 & 18,252 & \\
\hline$\%$ rural population & & $27.4 \%$ & $19.8 \%$ & $21.8 \%$ & \\
\hline $\begin{array}{l}\text { Total Imperial Valley } \\
\text { population }\end{array}$ & & 66,508 & 70,645 & 79,345 & \\
\hline $\begin{array}{l}\% \text { of Total population } \\
\text { in Imperial Valley }\end{array}$ & & $92.2 \%$ & $94.7 \%$ & $94.8 \%$ & \\
\hline
\end{tabular}

\section{REFERENCES}

1. United States Department of Commerce, Bursau of the Census, 1970 Cerau: Survey for Califomia Countiss.

2. J.E. Pick, Fopulation ana Eeonomie Statisties for Imperiat conty 19501970, unpublished draft report, U.C. Riverside (Apri1, 1976). 


\section{Section 9 \\ Geothermal Laws}

Jim Wharton and David Layton

\subsection{INTRODUCTION}

Development of geothermal resources in the Imperial Valley is guided by a series of statutes and regulations passed by the federal government, the State of California, and Imperial County. Although these laws apply in an overlapping manner, the primary law that applies to the development of geothermal resources depends on whether the land is private, federal, or state property. This section describes some of the important statutes and regulations that control geothermal development.

\subsection{FEDERAL LAW ON GEOTHERMAL RESOURCES}

The two major federal laws that apply to geothermal resources in the Imperial Valley are the Geothermal Steam Act of $1970^{1}$ and the Geothermal Energy Research, Development, and Demonstration Act of $1974 .^{2}$

\section{Federal Leases}

Federal laws existing before 1970 did not provide for the development of geothermal steam. The legislative history ${ }^{3}$ of the 1970 Act indicates that the intent of Congress was to provide statutory authority for the Secretary of the Interior to issue leases for the development of geothermal steam and the associated geothermal steam resources underlying public lands in much the same manner as he was authorized to lease land for development of oil and gas deposits under the Mineral Leasing Act of 1920, as amended: 4 The definitions under the mineral leasing laws were amended to include geothermal resources:

"Mineral leasing laws
shall mean the Geothermal.
Steam Act of 1970 which are
amendatory of or supplemen-
tary to any of the foregoing
Acts; Leasing Act minerals
sha11 mean all minerals which
are provided in the mineral
leasing laws and all geo-
thermal steam and associated
geothermal resources which,
upon the effective date of the
Geothermal Steam Act of
l970, are provided in that
Act to be disposed of there-
under." 5

The lands subject to geothermal leasing are specified in the Geothermal Steam Act. They consist of public, withdrawn, and acquired lands administered by the Forest Service in a national forest or other areas. Lands conveyed by the United States that are subject to a reservation of geothermal steam and associated resources are also available for leasing. ${ }^{6}$ The actual leasing of lands 
that are within a KGRA is done under a competitive bidding process as described in section 1003. A lease for such lands is awarded to the highest qualified bidder. Competitive bidding is not required when the lands to be leased are not in a KGRA. In that situation the lease is granted to the first qualified lease applicant. Section 1003 further provides for the conversion rights of leases under the Mineral Leasing Act of Acquired Lands. 7 According to the Act, no person can convert more than 10,240 acres of mineral leases, permits, applications, or mining claims. Conversion to a geothermal lease may on $1 y$ occur when an individual has shown, to the satisfaction of the Secretary of the Interior, that substantial expenditures have been made for exploration, development, or production "on the lands for which a lease is sought or on adjoining, adjacent, or nearby Federal or non-Federal 1ands". 7

Other lands open to competitive bidding are those that are within a KGRA and are subject to a right to conversion provided that,

"the competitive geothermal lease shall be issued to the person owning the right to conversion to a geothermal lease if he makes payment for an amount equal to the highest bona fide bid for the competitive geothermal lease, plus the rental for the first year, within thirty days after he receives written notice from the Secretary of the amount of the highest bid." 7

Restrictions on lease acreage and exemptions of certain federal lands are contained in Sections 1006 and 1014, respectively. Section 1006 limits a geothermal lease to 2,560 acres except where there are irregular subdivisions. Moreover, it restricts for most cases the total acreage a lessee can have in a particular state to 20,480 acres. Federal lands exempt from geotherma1 leasing under section 1014 include lands rithin a national recreation area, lands in a fish hatchery administered by the Secretary of the Interior, wildiffe refuge, wildife range, game range, wildlife management area, waterfowl production area, or lands acquired or reserved for the preservation of fish or vildife threatened with extinction.

Federe1 Regulations

The Department of the Interior has promulgated federal regulations that govern geothermal exploration and development activities related to leases granted under the Geothermal Steam Act. The rules governing geothermal leases give particular attention to environmental protection. Geothermal Resources Operational order (GRO) No. 4, for instance, states that a lessee must:

"Conduct exploration and development operations in 
a manner that provides maximum protection of the environment; rehabilitate disturbed lands; take all necessary precautions to protect the public health and safety; and conduct operations in accordance with the spirit and objectives of all applicable

Federal environmental legislation and supporting executive orders." 8

More specifically, GRO No. 4 requires a lessee to reclaim disturbed lands, reduce erosion, protect fish, wildlife, and their habitats, monitor subsidence and seismicity, comply with all applicable pollution control standards, and minimize noise. These and other conditions in the order are enforced by an Area Geothermal

Supervisor, who has authority to suspend operations on a lease that is in violation of regulations.

\section{Federal Geotherma1 Loan Guaranty}

Program

The Congress has established a loan guaranty program ${ }^{9}$ to encourage and assist in the commercial development of useful energy from geothermal resources by environmenta11y acceptable processes. The guaranty protects lenders against loss of principal or interest on loans made for the purposes of:

- The determination and evaluation of the resource base,

- research and development with respect to extraction and utilization technologies,
- acquisition of rights in geothermal resources, or

- development, construction, and operation of facilities for the demonstration or commercial production of energy from geothermal resources.

The amount of guaranty is limited to $75 \%$ of the aggregate cost of the project with respect to which the loan is made. In addition, the amount of the guaranty for any loan for a project shall not exceed $\$ 25,000,000$, and the amount of the guaranty for any, combination of loans for any single qualified borrower shall not exceed $\$ 50,000,000$.

A qualified borrower is any public or private agency, institution, association, partnership, corporation, political subdivision, or other legal entity that has presented satisfactory evidence of an interest in geothermal resources and is capable of performing research or completing the development and production of energy in an acceptable manner.

Any activities to develop geothermal resources shall place particular emphasis upon the objective of assuring that the environment and the safety of persons or property are effectively protected. All of the research, development, and demonstration functions, including the loan guaranty program, are vested in the 
Energy Research and Development Administration.

Federal Licenses and Permits

A possible area of federal control over geothermal power plants comes under the Federal Power Act. Although the Act applies generally to hydroelectric plants, the Federal Power Commission is authorized to regulate the interstate sales of electricity. ${ }^{10}$ Thus, any geothermal power transported out of the Imperial Valley to some other state would requirc prior approval by the comnission. The Federal Power Commission does not have the power to license use of surplus water by thermal electric plants. ${ }^{I I}$

The Corps of Engineers issues two permits that could be required by geothermal plants in the valley. $\Lambda$ construction permit under section 10 of the 1899 Rivers and Harbors Act is required to build any structure in navigable waters, 12 and a permit is needed to discharge refuse into certain waters under Section 13 of the same act. ${ }^{13}$ The corps, before issuing a permit, accepts the Environmental Protection Agency's (EPA) findings with respect to applicable water standards. ${ }^{14}$ The EPA transmits applications to state agencies near the facility. 15 Without a section 21 (b) ${ }^{16}$ certificate on the impact on water quality, a permit cannot be issued by the corps. Beside:; these water quality rerulation:, the EPA has other regulations derling with air pollution from new power plants. 17

\subsection{CALIFORNIA STATE LAUS ON: GEOTHERHAL RESOURCES}

The State of California has severa1 laws and regulations that will control geothermal development in the Imperial Valley. The main lum are the state geothermal lease lava, the state regulations of geothermal rells, and lawe governing porrer plants siting.

\section{State Geothermal Leases}

The issuance of leases for California state lands is generally controlled by statutes given in the California Public Resources Code section 6902 et seq. ${ }^{18}$ Leases for geothermal extraction and removal are issued by the State Lands Commission. State lands are defined as "al1 lands owned by the state, incluling school lands, proprietary lands, tidelands, submerged lands, swamp and overflowed lands, and beds of navigable rivers and lakes, and lands in which geothemal resources have been reserved to the state. "19 A lease application must be for at least 640 acres but not more than 2,560 acres of reasonably compact area. $^{20}$ A permit or lease, however, 
may be issued for less than 640 acres if the parcel is separated from other parcels of land available for permit or lease. 21 Section 6908 of the Public Resources Code also prohibits a person, association, or corporation from controlling directly or indirectly more than 25,600 acres of state geothermal leases. Competitive bidding is allowed on those lands that are classified as known geothermal resource areas, i.e., those areas that contain at least one well capable of producing geothermal resources in commercial quantities or are designated by the commission on the recommendation of the Geothermal Resources Board. The bidding is regulated by the State Lands Commission.

\section{Statutes on Geothermal We1ls}

The interest of the State of California in the drilling of geothermal wells is contained in the California Public Resources Code section 3700 et seq. ${ }^{22}$ The state's primary concern is "that wells for the discovery and production of geothermal resources be drilled, operated, maintained, and abandoned in such manner as to safeguard life, health, property, and the public welfare, and to encourage maximum economic recovery". 22

The following statutes from the California Public Resources Code provide for orderly development of geothermal resources, encourage the greatest possible economic recovery, and delegate to the State $0 i 1$ and Gas Supervisor the power to protect the environment:

Section 3714

"The State 011 and Gas Supervisor shall so supervise the drilling, operation, maintenance and abandonment of geothermal resources wells as to encourage the greatest ultimate economic recovery of geothermal resources,.... and to prevent damage to underground and surface waters suitable for irrigation or domestic purposes by reason of the drilling, operation, maintenance, and abandonment of geothermal resources wells."

Section 3715

"The supervisor shall also supervise the drilling, operation, maintenance, and abandonment of wells so as to permit the owners or operators of such wells to utilize all methods and practices known to the industry for the purpose of increasing the ultimate recovery of geotherma1 resources. . . . . . ."

Section 3724.1

"An owner or operator may submit to the supervisor for approval a written program to drill a shallow well or wells for geothermal observation purposes. In order to qualify under this section, a program shall contain not more than 25 wells and the maximum total depth of each of these wells sha11 not exceed 250 feet." 


\section{Section 3725}

"Every person who engages in the drilling, redrilling, or deepening of any well shall file with the supervisor an indemnity bond in the sum of five thousand dollars $(\$ 5,000)$ for each well drilled, redrilled, or deepened.

\section{Section 3730}
"The orner or operator of any well shall keep, or cause to be kept, a careful and accurate log, core record, and history of the drilling of the well."

Section 3739

\begin{abstract}
"Any person engaged in operating any wells wherein high pressures are known to exist, and any person drilling for geothermal resources in any district where the pressures are unknown shall equip the well with casings of sufficient strength, and with such other safety devices as may be necessary, in accordance with methods approved by the supervisor, and shall use every reasonable effort and endeavor effectually to prevent blow outs, explosions, and fires."
\end{abstract}

Callfornia Regulation of Geothermal Wel1s

State regulations as contained in the California Administrative Code are particularly concerned with blowout prevention, developmental wells, maintenance of drilling logs, and protection of the environment. These regulations are contained in
Title 14 of the California Administrative Code. $^{23}$ The general policy in drilling wells is to protect or minimize damages to the environment, usable: ground waters, geothormal resources, life, health, and propcrty. The regulations prescribe the notice requirements of the omer or operator of a geothermal resource. The foc: and bonds are given. In addition, requirements for well spacins, casing, construction of conductor pipe, mud return temperatures, and blowout-prevention equipment are contained in the regulations.

A log must be leept containing core records and a history of the drilling of the vell. The history must describe in detail, in chronological order, and on a daily basis all significant operitions carried out and equipment used during all phases of drilling, testing, completion, recompletion, and abandonment of the ve11. A summary report must accompany the core record and well history, shoring! data pertinent to the condition of a well at the time of completion of work done. Monthly production records must be filed with the supervisor on the 10th day of each month. Also, injection records must be filed with the superviosr.

The Administrative Code contains regulations pertinent to the subsidence that might occur in the 
Imperial Valley. These regulations are concerned with procedures for surveying and instaliing benchmarks for the detection of subsidence. The wells to be drilled must have benchmarks that tie into existing first or second order subsidence networks. Surveying must be coordinated with the County Surveyor and the actual work conducted under the supervision of a Registered Civi1 Engineer or Licensed Land Surveyor. Benchmarks must occur at accepted intervals and surveys of the benchmarks must be run annual1y at the expense of the operator while the well(s) are in production. 24

\section{Geotherma1 Powerplant Siting}

Powerplants operated by geothermal fluids will be licensed by the California Energy Resources Conservation and Development Commission. The energy commission has exclusive power to certify all powerplant sites and related facilities (e.g., transmission lines). Section 25500 of California Public Resources Code states that "the issuance of a certificate by the commission shall be in lieu of any permit, certificate, or similar document required by any state, local or regional agency, or federal agency to the extent permitted by federal law...." 25 However, there is still a Certificate of Public Convenience needed from the Public Utilities Commission. 26

Areas prohibited as powerplant sites are parks, wilderness, scenic or natura1 reserves, and areas for wildlife protection, recreation, or historic preservation. 27 An applicant may be required to obtain development rights in the area of a proposed site so that local population densities can be controlled. 28 In addition, a special monitoring system, to be run by the commission in cooperation with other state and local agencies, must be used to verify compliance with the applicable environmental regulations. 29

\section{Section 25540 specifically} exempts an applicant from analyzing 3 alternative sites for a proposed geothermal energy facility. Under section 25541 of the code a powerplant under $100 \mathrm{MW}$ (including a geothermal plant) may be exempted from the certification process if the commission finds that:

- "No substantial adverse impact on the environment or energy resources will result from the construction or operation of the proposed facility or from modification", and

- "Generating capacity will not be added which is substantially in excess of the forecast of electrical energy demands..." 30 
If the powerplant is below $50 \mathrm{MN}$, the Energy Conservation and Development Act does not apply.

9.4 LOCAL LAWS OF IMPERIAL COUNTY Additional regulations affecting geothermal activities in the valley have been adopted by Imperial County. Those regulations are described in the document, "Terms, Conditions, Standards, and Application Procedures for Initial Geothermal Development, Imperial County," which was issued in May of 1971 by the Department of Public Works. The terms and conditions are designed to encourage the orderly development of initial geothermal facilities, but are only interim in nature because a more comprehensive plan dealing: with geothermal resources is being developed.

The regulations stipulate, amon: other things, that geothermal operitors must comply with al 1 appropriate local, state, and federal lavs durin: the different phases of exploration and development. Furthermare, operators of a geothermal production project must monitor subsidence, minimi:ze noise, preserve farm land, and abandon project sites according to prescribed procedures. Zoning ordinances are another form of county regulation. Production projects may only develop geothermal resources within areas specified by the County Planning Commission.

$\mathrm{BKI} / \mathrm{gw} / \mathrm{vt}$ 
1. 30 United States Code, Sec. 1001 et seq.

2. 30 United - aates Code, Sec. 1101 et seq.

3. Interior and Insular Affairs Committee, House Report No. 19-1544, 91st. Congress, 2nd. Session (1970); 3 U.S. Congressional and Administrative News, 5113 (1970).

4. 30 United States Code, Sec. 181 et seq.

5. 30 United States Code, Sec. 530.

6. 30 United States Code, Sec. 1002.

7. 30 United States Code, Sec. 1003.

8. United Stated Department of the Interior, Geological Survey, Geothermal Resources Operational Orders, January, 1976.

9. 30 United States Code, Sec. 1411-1144.

10. 16 United States Code, Sec. 796.

11. Chemehuevi Tribe of Indians V. Federal Power Commission, 955 S. Ct. 1066, Dist. Col. 1975.

12. 33 United States Code, Sec. 403 (1970).

13. 33 United States Code, Sec. 407 (1970).

14. Executive order 11574, 2 (a) (2A), 3c, F, R, 556 (1971).

15. Water Pollution Control Act section 21(b), 33 United States Code, Sec. 1171 (b) (1970).

16. See Note 15 , above.

17. 42 United States Code, Sec. 1857C-6(b) (1970).

18. West's Calffornia Public Resources Code, Sec. 6902 et seq.

19. West's California Public Resources Code, Sec. 6904.

20. West's California Public Resources Code, Sec. 6908.

21. West's California Public Resources Code, Sec. 3700 et seq.

22. Id. Sec. 3700 .

23. California Administrative Code, Title 14, Sec. 1900 et seq.

24. Id. Sec. 1971.

25. West's California Public Resources Code, Sec. 25500.

26. West's California Public Utilities Code, Sec. 1001; West's California Public Resources Code, Sec. 25518.5.

27. West's California Public Resources Code, Sec. 25527.

28. Id. Sec. 25528.

29. Id. Sec. 25522 .

30. Id. Sec. 25120 . 